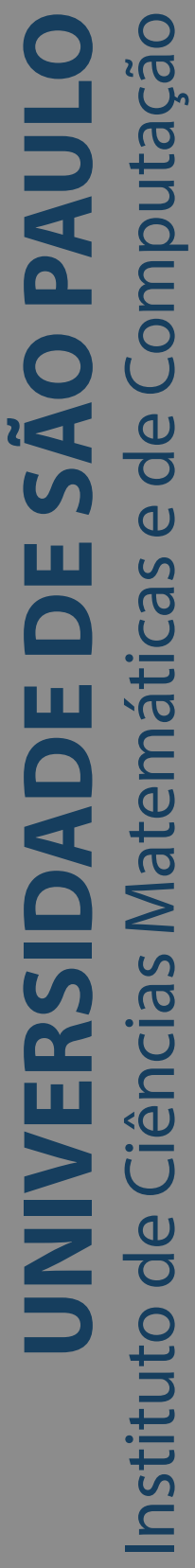

\title{
Automatic generation of configurable test-suites for software
} product lines

\section{Vanderson Hafemann Fragal}

Tese de Doutorado do Programa de Pós-Graduação em Ciências de Computação e Matemática Computacional (PPG-CCMC) em dupla titulação com Högskolan I Halmstad 



\section{Vanderson Hafemann Fragal}

\section{Automatic generation of configurable test-suites for software product lines}

Doctoral dissertation submitted to the Institute of Mathematics and Computer Sciences - ICMC-USP, in partial fulfillment of the requirements for the degree of the Doctorate Program in Computer Science and Computational Mathematics and in double degree with Högskolan I Halmstad. FINAL VERSION

Concentration Area: Computer Science and Computational Mathematics

Advisor: Prof. Dr. Adenilso da Silva Simão 
Ficha catalográfica elaborada pela Biblioteca Prof. Achille Bassi e Seção Técnica de Informática, ICMC/USP, com os dados inseridos pelo(a) autor(a)

Fragal, Vanderson Hafemann Automatic generation of configurable test-suites for software product lines / Vanderson Hafemann Fragal; orientador Adenilso da Silva Simão. -- São Carlos, 2018 . $149 \mathrm{p}$.

Tese (Doutorado - Programa de Pós-Graduação em Ciências de Computação e Matemática Computacional) Instituto de Ciências Matemáticas e de Computação, Universidade de São Paulo, 2018.

1. Software Product Line. 2. Model-Based Testing. 3. Test Generation. 4. Full Fault Coverage. I. Simão, Adenilso da Silva, orient. II. Título. 


\section{Vanderson Hafemann Fragal}

\section{Geração automática de conjuntos de teste configuráveis para linhas de produto de software}

Tese apresentada ao Instituto de Ciências Matemáticas e de Computação - ICMC-USP, como parte dos requisitos para obtenção do título de Doutor em Ciências - Ciências de Computação e Matemática Computacional e em dupla titulação com Högskolan I Halmstad. VERSÃO REVISADA

Área de Concentração: Ciências de Computação e Matemática Computacional

Orientador: Prof. Dr. Adenilso da Silva Simão 

First of all, I would like to thank my advisors Prof. Adenilso Simao and Prof. Mohammad Reza Mousavi for excellent guidance. Thanks for encouraging and challenging me and also for good cooperation. I would also like to thank my other colleagues at the department of computer science and especially the software engineering research group for the inspiring and developing work environment. Thanks to all who have participated in focus group meeting and questionnaires. Finally, thanks to my family, my parents, my brother, and sisters for constant encouragement. 



\section{ABSTRACT}

FRAGAL, V. H. Automatic generation of configurable test-suites for software product lines. 2018. 149 p. Tese (Doutorado em Ciências - Ciências de Computação e Matemática Computacional) - Instituto de Ciências Matemáticas e de Computação, Universidade de São Paulo, São Carlos - SP, 2018.

Software Product Line Engineering (SPLE) is an approach used in the development of similar products, which explores the systematic reuse of software artifacts. The SPLE process has several activities executed to ensure software quality. Quality assurance is of vital importance for achieving and maintaining a high quality of all kinds of artifacts, such as products and processes. Testing activities are widely used in the industry for quality management. However, the effort for applying testing is usually high, and increasing the testing efficiency is a major concern of all systems engineering activities. A common means of increasing efficiency is automation of the test execution and the test design. Automated test design can be performed using approaches such as Model-Based Testing (MBT) in which the real behavior of a software system is compared to an abstract test model. Several techniques, processes, and strategies were developed for SPLE testing, but still many problems are open in this area of research. The challenge in focus is the reduction of the overall test effort required to test SPLE products. Test effort can be reduced by maximizing test reuse using models that take advantage of the similarity between products. The thesis goal is to automate the generation of small test-suites with high fault detection and low test redundancy between products. To achieve the goal, equivalent tests are identified for a set of products using complete and configurable test-suites. Two research directions are explored, one is product-based centered, and the other is product line-centered. For test design, test-suites that have full fault coverage were generated from state machines with and without feature constraints. A prototype implementation tool was developed for test design automation. In addition, the proposed approach was evaluated using examples, experimental studies, and an industrial case study for the automotive domain. The results indicates test effort reduction of $36 \%$ in the first research direction for a product line with 24 products, and in the second research direction increasing test effort reduction based on the number of products that require testing. For 6 products $15 \%$ reduction (from case study), and for 20 random products $50 \%$ reduction (from experimental studies).

Keywords: Software Product Line, Model-Based Testing, Test Generation, Full Fault Coverage. 



\section{RESUMO}

FRAGAL, V. H. Geração automática de conjuntos de teste configuráveis para linhas de produto de software. 2018. 149 p. Tese (Doutorado em Ciências - Ciências de Computação e Matemática Computacional) - Instituto de Ciências Matemáticas e de Computação, Universidade de São Paulo, São Carlos - SP, 2018.

Engenharia de Linha de Produto de Software (SPLE) é uma abordagem utilizada no desenvolvimento de produtos similares, que explora a reutilização sistemática de artefatos de software. O processo da SPLE executa várias atividades para garantir a qualidade do software. Atividades de garantia de qualidade são fundamentais para alcançar e manter altos níveis de qualidade em todos os tipos de artefatos de software, tais como produtos e processos. Atividades de teste são amplamente utilizadas na indústria para o gerenciamento de qualidade. No entanto, o esforço para a aplicação de testes geralmente é alto e melhorar a eficiência dos testes é um desafio relacionado a todas as atividades da engenharia de sistemas. Uma maneira de melhorar a eficiência da atividade de teste é automatizar a geração e execução dos testes. A geração automática de testes pode ser realizada por abordagens tais como o Teste Baseado em Modelos (TBM), em que o comportamento real do sistema de software é comparado a um modelo de teste abstrato. Várias técnicas, processos e estratégias foram desenvolvidas para o teste de SPLE, contudo, existem diversos desafios nessa área de pesquisa. O desafio em foco é a redução do esforço geral de teste necessário para testar produtos da SPLE. O esforço de teste pode ser reduzido maximizando o reuso de teste usando modelos que representam variabilidades entre os produtos. O objetivo da tese é automatizar a geração de compactos conjuntos de testes com alta capacidade de detecção de falhas e baixa redundância de teste entre produtos. Para alcançar tal objetivo, testes equivalentes são identificados para um conjunto de produtos usando conjuntos de teste completos e configuráveis. Duas direções de pesquisa são exploradas, uma centrada no produto e a outra centrada na linha de produto. Foram gerados conjuntos de teste que tenham cobertura de falhas completa a partir de máquinas de estado com e sem restrições de características. A implementação de uma ferramenta foi desenvolvida para automatizar a geração de teste. Além disso, a abordagem proposta foi avaliada usando exemplos, estudos experimentais e um estudo de caso industrial. Os resultados indicam uma redução de esforço de teste de $36 \%$ na primeira direção de pesquisa para uma linha com 24 produtos, e na segunda linha de pesquisa uma redução incremental com mais produtos a serem testados. Para 6 produtos uma redução de 15\% (do estudo de caso), e para 20 produtos randomicos uma redução de $50 \%$ (dos estudos experimentais).

Palavras-chave: Linha de Produto de Software, Teste Baseado em Modelos, Geração de Teste, Cobertura de Falhas Completa. 

Figure 1 - Directions of research in SPL test design. . . . . . . . . . . . . . 22

Figure 2 - Directions of research and chapters. . . . . . . . . . . . 23

Figure 3 - Testing terms in Software Engineering. . . . . . . . . . . . . . 26

Figure 4 - The V-model (adapted from IT-Beauftragten (1997)) . . . . . . . . . . . 28

Figure 5 - Application fields of model-based testing (adapted from Weissleder

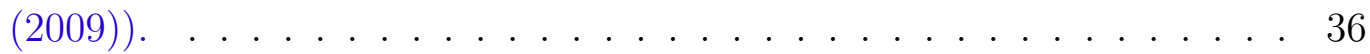

Figure 6 - Model-based testing overview (adapted from Pretschner and Philipps

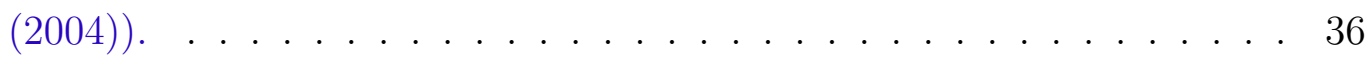

Figure 7 - Abstract FSM $M . \ldots \ldots \ldots \ldots \ldots$

Figure 8 - Testing tree of a transition cover set. . . . . . . . . . . . . . . 43

Figure 9 - Software product line development process (adapted from Pohl, Böckle and Linden (2005)). . . . . . . . . . . . . . . . . . . 48

Figure 10 - AGM Feature Model (adapted from SEI (2011)). . . . . . . . . . . . . 49

Figure 11 - Satisfiable product configurations of AGM. . . . . . . . . . . . . 50

Figure 12 - The W-model for SPL testing (adapted from Jinhua, Qiong and Jing

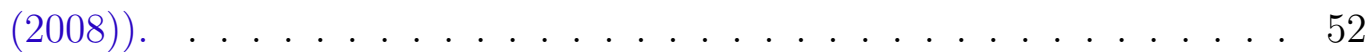

Figure 13 - 3D SPL test process model with dimensions of evolution (adapted from Engstrom and Runeson (2013)) . . . . . . . . . . . . . . 53

Figure 14 - Classic SPL testing strategies applied to the 3D model (adapted from Runeson and Engstrom (2012)) . . . . . . . . . . . . 55

Figure 15 - SPL testing artifacts interaction (adapted from McGregor (2001)) . . . 56

Figure 16 - Proposed regression-based test reuse strategy. . . . . . . . . . . . . . 61

Figure 17 - Algorithm for reducing an $n$-complete test-suite. . . . . . . . . . . 64

Figure 18 - FSM of the third product configuration of AGM. . . . . . . . . . 66

Figure 19 - Example of regression-based test sets. . . . . . . . . . . . . . . . . 67

Figure 20 - FFSM for the AGM SPL . . . . . . . . . . . . . . . . . 71

Figure 21 - Alternative FFSM for the AGM SPL . . . . . . . . . . . . . . 71

Figure 22 - Abstracted FFSM of AGM. . . . . . . . . . . . . . . 79

Figure 23 - Conditional state tree for AGM. . . . . . . . . . . . . . . 80

Figure 24 - Conditional transition tree for AGM. . . . . . . . . . . . . . . . . . . 82

Figure 25 - HFSM for AGM. . . . . . . . . . . . . . . . . . . . . . 91

Figure 26 - State structure for AGM HFSM. . . . . . . . . . . . . . . . . . . 92

Figure 27 - Simple composition of regions states of a CompAnd state. . . . . . . . 100 
Figure 28 - HFSM composition of regions states of a CompAnd state. . . . . . . 101

Figure 29 - SPL validation workflow using HFSMs. . . . . . . . . . . . . . . . . 101

Figure 30 - HFSM for AGM SPL on the implemented tool. . . . . . . . . . . . 106

Figure 31 - Textual FFSM for AGM SPL on ConFTGen. . . . . . . . . . . . . . . 107

Figure 32 - Derived HFSM for AGM SPL . . . . . . . . . . . . . . . . . 107

Figure 33 - Semantic FFSM of the derived HFSM. . . . . . . . . . . . . . . . 108

Figure 34 - SMT parts for checking conditional states and the complete property. . 109

Figure 35 - HFSM for AGM SPL with deterministic error. . . . . . . . . . . . . . 110

Figure 36 - SMT parts for checking a conditional prefix relation. . . . . . . . . . . 111

Figure 37 - Mobile Media feature model and derived products. . . . . . . . . . . . 114

Figure 38 - FSM of the first product of Mobile Media. . . . . . . . . . . . . . . 115

Figure 39 - Accumulated effort per designed product. . . . . . . . . . . . . 116

Figure 40 - Test-suite size of the core specification. . . . . . . . . . . . . . . . 120

Figure 41 - Number of new tests for an FFSM and FSMs. . . . . . . . . . . . . . . 121

Figure 42 - Time required to execute the HSI method for one FFSM and some FSMs.122

Figure 43 - Number of FFSM conditional transitions per kind of feature model. . . 123

Figure 44 - Configurable test-suite size per kind of feature model. . . . . . . . . . . 124

Figure 45 - Time required to execute the extended HSI method per kind of feature model. . . . . . . . . . . . . . . . . . . . . . 125

Figure 46 - Adapted Feature Model of the Body Comfort System (LITY et al., 2015).127

Figure 47 - HFSM of 4 composed components of BCS. . . . . . . . . . . . . 127

Figure 48 - Overview of the region composition of BCS HFSM. . . . . . . . . . . . 128

Figure 49 - Feature Model configuration selection for BCS. . . . . . . . . . . . . . 129

Figure 50 - HFSM derived for 3 configurations with 3 composed components. . . . 129

Figure 51 - HFSM derived for one configuration and 2 composed components. . . . 130

Figure 52 - Example of redundancy on SPL test design. . . . . . . . . . . . . . . 132 


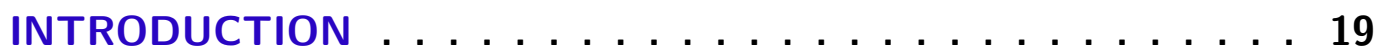

1.1 Motivation . . . . . . . . . . . . . . . . . . 19

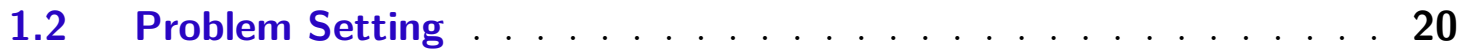

1.3 Thesis Statement . . . . . . . . . . . . . . . . . 21

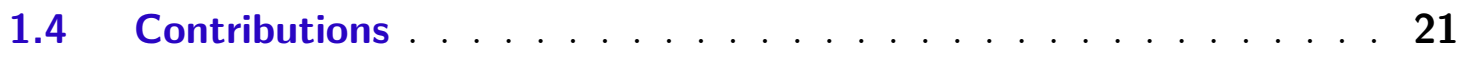

1.5 Outline ........................ 23

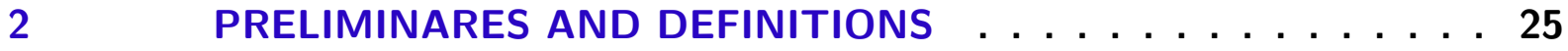

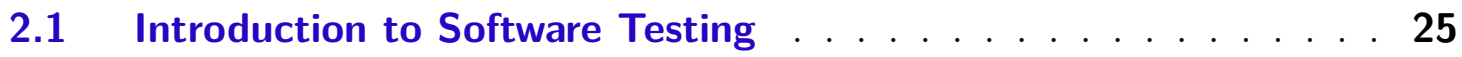

2.1.1 Faults and Test-cases . . . . . . . . . . . . . 26

2.1.2 Test Process and Phases . . . . . . . . . . . . . . . . 27

2.1.3 Regression Testing . . . . . . . . . . . . . . . . . . 29

2.1.4 Coverage Criteria . . . . . . . . . . . . . . . . . . . 31

2.1.5 Test Techniques. . . . . . . . . . . . . . . . . . . . 32

Functional testing . . . . . . . . . . . . . . . 32

Structural testing . . . . . . . . . . . . . . . 33

Error-based testing . . . . . . . . . . . . . . . . . . . 34

2.1.6 Test Automation and Quality . . . . . . . . . . . . . 34

2.2 Model Based Testing . . . . . . . . . . . . . . . . . . 35

2.2.1 Model-Based Testing Process . . . . . . . . . . . . . . . . 35

2.2.2 Test-case Concretization . . . . . . . . . . . . . . . . . 37

2.2.3 Model-Based Testing Techniques. . . . . . . . . . . . . . 38

2.2.4 Test-case Generation for Finite State Machines . . . . . . . . . 39

Finite State Machines . . . . . . . . . . . . . . . . . . . . 40

Validation Properties . . . . . . . . . . . . . . . . . . . . 40

Test-cases . . . . . . . . . . . . . . . . . 41

State Coverage Criteria . . . . . . . . . . . . . . . . 42

Transition Coverage Criteria . . . . . . . . . . . . . . . . . . 42

Full Fault Coverage Criteria . . . . . . . . . . . . . . . . . . . 43

Test-case Generation Methods . . . . . . . . . . . . . . . . . . . . . . 44

2.3 Software Product Lines . . . . . . . . . . . . . . . . . 46

2.3.1 Development Process . . . . . . . . . . . . . . . . . 47 
2.3.2 Feature Model . . . . . . . . . . . . . . . . . . . . 48

2.3.3 Software Product Line Testing . . . . . . . . . . . . . . . . . . . 51

Test Process Model . . . . . . . . . . . . . . . . . . . . . . . . . . . . 51

Dimensions of Evolution . . . . . . . . . . . . . . . . . . . . . . . 52

Test Strategies . . . . . . . . . . . . . . . . . . . . . 53

Regression-based SPL Testing . . . . . . . . . . . . . . . 55

2.4 Concluding Remarks . . . . . . . . . . . . . . . . . . . 55

REGRESSION-BASED TESTING FOR SOFTWARE PRODUCT LINES 59

3.1 Test Effort Calculation . . . . . . . . . . . . . . . . . 59

3.2 Regression-based Testing for Software Product Lines . . . . . . . 60

3.2.1 Selection Algorithm . . . . . . . . . . . . . . . . . . 61

3.2.2 Test-suite Analysis . . . . . . . . . . . . . . . . . . . . . 65

3.3 Test Design Example . . . . . . . . . . . . . . . . 66

3.4 Concluding Remarks . . . . . . . . . . . . . . . . . . . 68

4 FEATURED FINITE STATE MACHINES . . . . . . . . . 69

4.1 Syntax and Semantics . . . . . . . . . . . . . . . . . 69

4.2 Model Derivation . . . . . . . . . . . . . . . . . 71

$4.3 \quad$ Validation Properties . . . . . . . . . . . . . . . . . 72

4.3.1 Determinism . . . . . . . . . . . . . . . . . . . . . 73

4.3 .2 Complete . . . . . . . . . . . . . . . . . 74

4.3.3 Initially Connected . . . . . . . . . . . . . . . . . . 75

4.3 .4 Minimal . . . . . . . . . . . . . . . . . . . . . . . 76

4.4 Test Design . . . . . . . . . . . . . . . . . . . . . . . . 77

4.4.1 Configurable Test-suites . . . . . . . . . . . . . . . . 77

4.4.2 Test-case Derivation . . . . . . . . . . . . . . . . . . . . . . . . 77

4.4.3 State Coverage . . . . . . . . . . . . . . . . . . . . . . . 78

4.4.4 Transition Coverage . . . . . . . . . . . . . . . . . . . 80

4.4.5 Full Fault Coverage . . . . . . . . . . . . . . . . . . . . . 83

Parametrized Separating Set . . . . . . . . . . . . . . . . . . 83

State Identification . . . . . . . . . . . . . . . . . . . . . . . . 85

Extended HSI Method . . . . . . . . . . . . . . . . . . . . . . . . 86

4.5 Concluding Remarks . . . . . . . . . . . . . . . . . . 87

5 HIERARCHICAL FEATURED STATE MACHINE . . . . . . . 89

5.1 Syntax . . . . . . . . . . . . . . . . 90

5.1.1 Well-formed state structure . . . . . . . . . . . . . . . . . 91 
5.1.2 Well-formed transitions . . . . . . . . . . . . . . 95

5.2 Semantics . . . . . . . . . . . . . . . . . . . . 97

5.2.1 Mapping HFSM to FFSM . . . . . . . . . . . . . . . . . . 97

5.2.2 Composition of Orthogonal States . . . . . . . . . . . . . . . . . 99

5.3 Validation and Test Generation . . . . . . . . . . . . . . 100

5.4 Concluding Remarks . . . . . . . . . . . . . . . . . . . . . . . 102

$\begin{array}{lll}\text { III } & \text { APPLICATION }\end{array}$

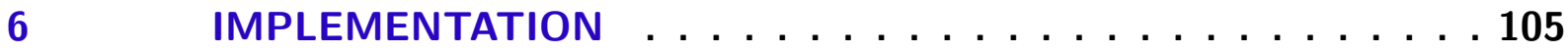

6.1 Configurable Full Test Generator Tool . . . . . . . . . . . . . 105

6.1 .1 Model Derivation . . . . . . . . . . . . . . . . . . . . . 106

6.1 .2 Model Validation . . . . . . . . . . . . . . . . . . 108

6.1.3 Prefix Relation Check . . . . . . . . . . . . . . . . . . 110

6.1.4 Test Generation . . . . . . . . . . . . . . . . . . . . . . 111

6.2 Concluding Remarks . . . . . . . . . . . . . . . . . . 112

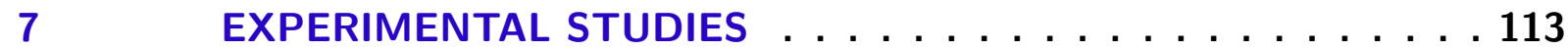

7.1 Incremental Regression-based Approach . . . . . . . . . . . . 113

7.1.1 Experimental Setup . . . . . . . . . . . . . . . . 113

7.1.2 Analysis of Results and Discussion . . . . . . . . . . . . . . . . 115

7.2 Random Featured-based Test Model . . . . . . . . . . . . . . . 117

7.2.1 Experimental Setup . . . . . . . . . . . . . . . . . . . 118

7.2.2 Analysis and Threats to Validity . . . . . . . . . . . . . . 119

Q1- Is there a difference between generating a test-suite for an FSM and pruning a configurable test-suite for the same FSM? . . . . . . . 119

Q2- In which scenario do we reduce the number of test-cases using an FFSM instead of FSMs? . . . . . . . . . . . . . . . . . . 120

Q3- In which scenario do we have smaller test generation times using an FFSM instead of FSMs? . . . . . . . . . . . . . . . . 122

Q4- Is there a relation between the feature model and the number of conditional transitions? . . . . . . . . . . . . . . . . . 123

Q5- Is there a relation between the feature model and and the configurable test-suite size ? . . . . . . . . . . . . . . . . . . 124

Q6- Is there a relation between the feature model and the extended HSI test generation time?. . . . . . . . . . . . . . . . 125

7.3 Body Comfort System Case Study . . . . . . . . . . . . . . . 126

7.3.1 Behavior Modeling . . . . . . . . . . . . . . . . . . . 126

7.3 .2 Model Derivation . . . . . . . . . . . . . . . . . . . . 128 
7.3.3 Test Generation . . . . . . . . . . . . . . . . . . . . . . 130

7.4 Test Redundancy Problem . . . . . . . . . . . . . . . . . 130

7.5 Concluding Remarks . . . . . . . . . . . . . . . . . 132

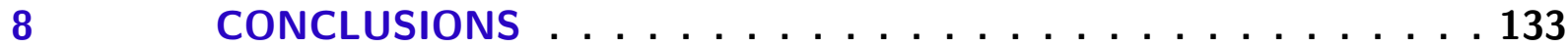

8.1 Summary of Contributions . . . . . . . . . . . . . . 134

8.2 Related Work . . . . . . . . . . . . . . . . 135

8.3 Publications . . . . . . . . . . . . . . . . . . . . . . 138

8.4 Future Work . . . . . . . . . . . . . . . . . . . . 138

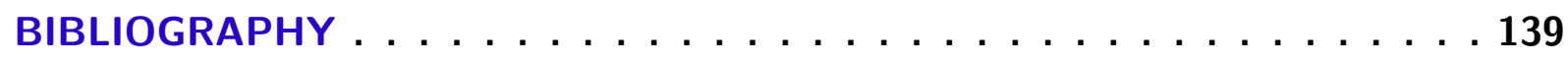

Glossary 
Part I

Background 

CHAPTER

1

INTRODUCTION

\subsection{Motivation}

Over the years, the skill of individual developers was not enough to develop large software systems and meet client requirements. The huge overload of the means of production turned into an ineffective development process. In the face of the increasing complexity, software industries moved from craftsmanship to industrialization where they customize and assemble components to produce similar products with low cost and satisfying several customer demands (GREENFIELD; SHORT, 2003).

Software design has evolved, and new requirements for custom/extensible software emerged while the expected release time was reduced. To satisfy such necessities new approaches in the software engineering appeared. Software Product Line Engineering (SPLE) is a paradigm to develop software where a family of related products (a Software Product Line - SPL) is built out of a common set of core assets, thus reducing development costs for each product (POHL; BÖCKLE; LINDEN, 2005). In SPLE, products are built, step-by-step, by incrementally adding or removing functionalities, which alleviate software complexity and improve quality (LINDEN; SCHMIF; ROMMES, 2007).

The SPL development process uses a product line architecture to perform a systematic reuse on requirements, architecture artifacts, components, and tests separated into two levels: domain engineering and application engineering. The domain engineering is product line-centered which develop the product line architecture with reusable/configurable artifacts. The application engineering is product-centered which develop products by instantiating the product line architecture (LINDEN; SCHMIF; ROMMES, 2007).

Similar to the development of single systems, the SPLE process also has several activities that are executed to ensure software quality. Verification, validation and testing are examples of such activities that are useful to check software functionalities and minimize 
risks. Testing is a very important activity in the software engineering to detect faults in the software system (SCHIRMEIER; SPINCZYK, 2009). Despite the systematic software artifact reuse that increases productivity, new challenges arise in the testing activity for SPLE.

Testing activities represent a large share of overall project costs and are even more challenging in SPLE than for single systems (TEVANLINNA; TAINA; KAUPPINEN, 2004). Unfortunately, several domains such as embedded and safety-critical systems do not strictly follow development standards to test several product configurations efficiently in a systematic manner. For example, the standard ISO $26262^{1}$ for safety-critical automotive software states that each developed product configuration should be tested using modelbased techniques with a high degree of test coverage under some test criteria (CICHOS et al., 2011).

Several techniques, processes, and strategies (UZUNCAOVA et al., 2008; WEISSLEDER; SOKENOU; SCHLINGLOFF, 2008; OSTER; MARKERT; RITTER, 2010; LOCHAU et al., 2012; OSTER et al., 2012a) were developed for SPLE, but still many problems are open in this area of research. First of all, testing every single product configuration individually by using common testing techniques is not acceptable for large SPLs. In general, testing products on-demand is unacceptable, due to the scarce time available for product assembly and testing a newly instantiated product. In addition, there are other challenges in SPLE including artifact management and test redundancy (RUNESON; ENGSTROM, 2012; ENGSTROM; RUNESON, 2013).

This thesis is focused on reducing test redundancy of functional conformance model-based testing for SPLs. Functional conformance testing compares a software system to an abstract specification to check whether the expected behavior does match. The Model-Based Testing (MBT) approach (OSTER et al., 2012b) can automate the test process using a formal test model. By automating the testing process, project costs are reduced, requirements have evolution support, and tests can achieve high fault detection rate (LASALLE; PEUREUX; GUILLET, 2011).

\subsection{Problem Setting}

This thesis explores the problem of test redundancy in SPL and proposes a solution such that functional requirements are expressed by test models based on state machines for test design. The main research question is:

Is it possible to develop a test design method that provides low test redundancy and high fault detection for an SPL?

$\overline{1 \quad \text { https://www.iso.org/standard/43464.html }>~}$ 
Test artifacts provide low redundancy when there are few different but equivalent elements that have the same purpose. Test artifacts that have high fault detection capability satisfy some strong test criteria, and preferably that leads to reasonable test costs. The main test artifacts produced in our solution are configurable test models (based on $150 \%$ models (GRÖNNINGER et al., 2008)) and configurable test-suites. A configurable test model can represent the whole functional behavior of an SPL, and both configurable testsuites and test models can be instantiated using product configurations. Configurable test artifacts can use feature-based product configurations of the SPL to derive test artifacts by pruning elements (negative variability) (CICHOS et al., 2011; KAMISCHKE; LOCHAU; BALLER, 2012; CLASSEN et al., 2013).

\subsection{Thesis Statement}

A test design method can be developed to create reusable functional test artifacts that provide low test redundancy and high fault detection for an SPL, in which these artifacts can be configured to test a set of products derived from the SPL.

\subsection{Contributions}

Several contributions lead to the proposed solution. This thesis focuses on a functional model-based testing approach for SPLs to reduce test-case redundancy, in two directions. Figure 1 provides an abstract overview of the two directions of research presented in this thesis. In the first research direction, the test design is focused on the product while the second direction focuses on the product line architecture with configurable artifacts for behavioural conformance (FISCHBEIN; UCHITEL; BRABERMAN, 2006). Dashed arrows represent the dependencies/derivation of artifacts of each step. The flow begins at the requirements and finishes at the derived test-suite. Both directions (starting in [1] and [2]) can derive test-suites. However, one direction may achieve a better relation between cost and benefit.

In the first direction, we explore a test-case reuse strategy named Incremental Regression-based Testing for Software Product Lines (IRT-SPL). The IRT-SPL can reduce test costs of a new derived product in the advanced development stages based on regression testing and the P method (SIMAO; PETRENKO, 2010). This research direction enables the efficient reuse of test-cases where only a few existing test-cases are selected and incremented to test a new derived product using fewer resources. The IRT-SPL strategy has three main contributions:

- an incremental test-case reuse strategy; 


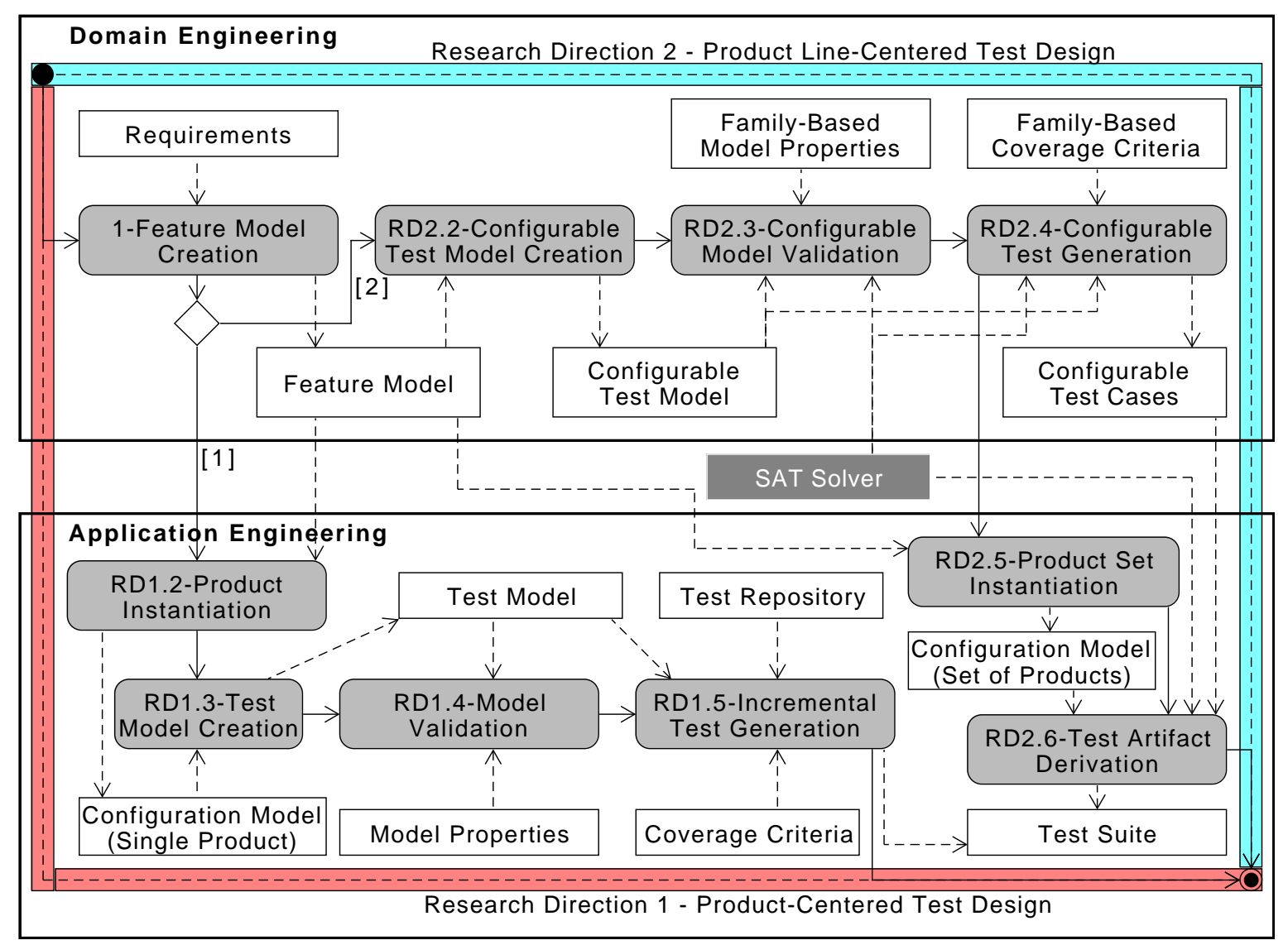

Figure 1 - Directions of research in SPL test design.

- a test-case selection algorithm; and

- experimental evaluation using a case study.

In the second direction, we explore test design in the early development stages. Specifically, we propose a solution named Configurable Feature-based full Coverage testing of State Machines (CFC-SM). The CFC-SM approach has some contributions:

- proposing new configurable family-based test models for SPL;

- proposing family-based validation criteria for the full fault coverage criteria and proving them to coincide with their product-based counterparts;

- proposing the extension of the HSI test-case generation method and proving them to coincide with their product-based counterparts;

- implementing a model-based test generation tool with a graphical interface to support validation, derivation, and generation of family-based test artifacts; and

- experimentally evaluating using a realistic SPL as a case study. 


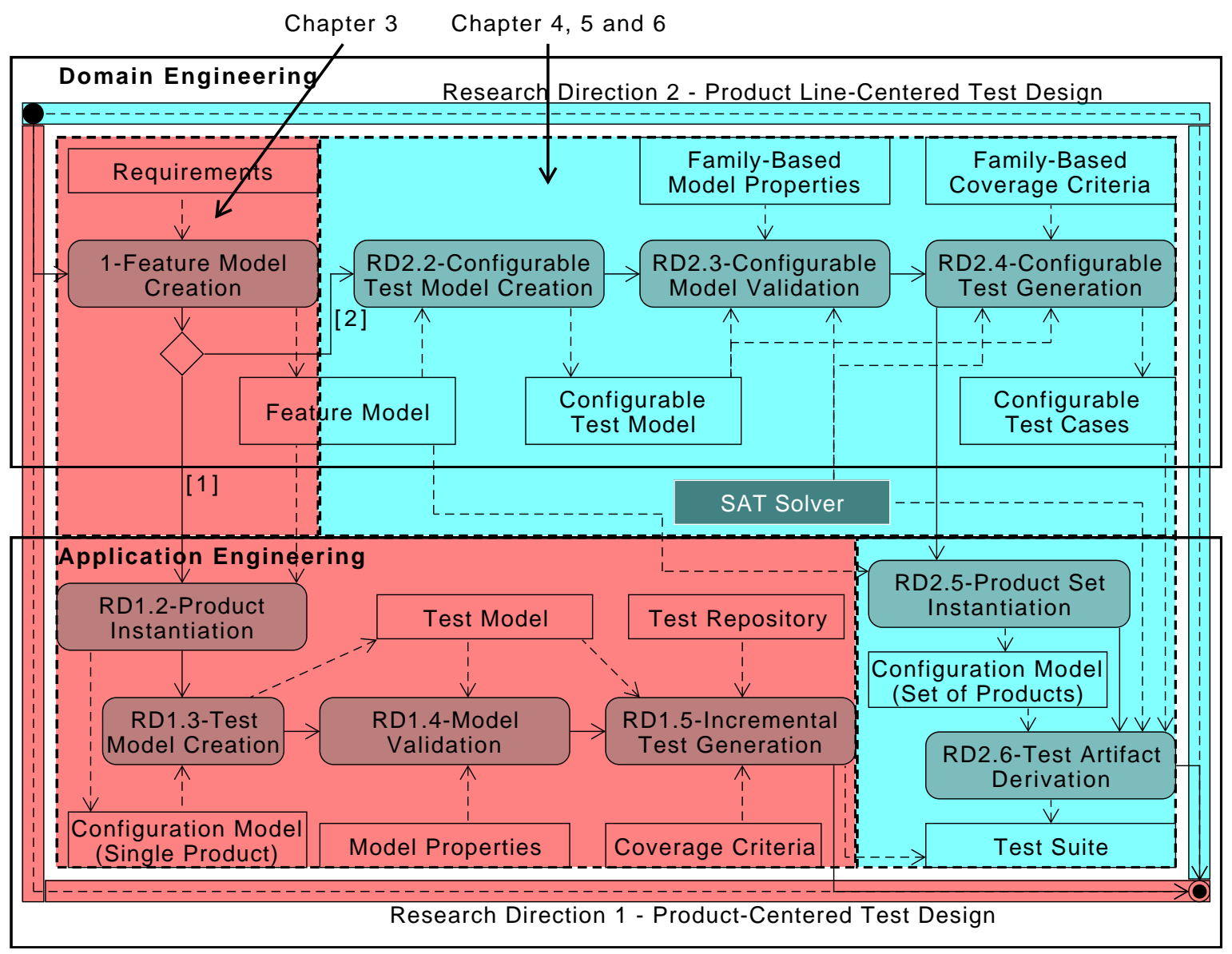

Figure 2 - Directions of research and chapters.

\subsection{Outline}

The thesis is structured as follows. In Chapter 2, preliminaries of the thesis are presented such as software testing, model-based testing, and SPLs. Chapter 3 presents the IRT-SPL strategy. Chapter 4 presents the configurable test model which is proposed for SPLs. Chapter 5 presents the hierarchical version of the configurable test model that is proposed as an alternative to the previous model. On Chapter 6 we introduce the implemented tool ConFTGen that provides graphical support for the derivation of test artifacts, and also our usage of a SAT solver. Chapter 7 presents experimental results and a case study for the proposed solutions. Finally, we conclude the thesis in Chapter 8.

Figure 2 depicts the relation of chapters presented with the explored research directions in this thesis. 



\section{PRELIMINARES AND DEFINITIONS}

This chapter contains preliminaries and basic definitions of the thesis that are required to understand further concepts.

The remainder of this chapter is organized as follows. Section 2.1 introduces basic concepts of software testing for test design, while Section 2.2 presents concepts with regard to model-based testing and test design automation. Finally, Section 2.3 presents basic notions and testing concepts about software product lines.

\subsection{Introduction to Software Testing}

The software development process has several activities, techniques, tools and methods that may be used to increase software quality. Verification, Validation, and Testing (VV\&T) activities are used to minimize software risks and errors. Verification checks whether the results obtained during a development phase satisfy the requirements established for that phase (MYERS et al., 2004). Validation checks whether the developed software (program) satisfies all requirements (MYERS et al., 2004). Testing detects the presence of faults in the software by observing its execution (MYERS et al., 2004). Testing can also be used for verification and validation (AMMANN; OFFUTT, 2008). Results obtained in testing activity are also useful for maintenance and debugging. The maintenance process releases new versions of the developed software by performing updates to fix functionalities. During maintenance, regression testing can be used to verify whether new faults were not inserted after performing software modifications. On the other hand, the debugging process aims at finding faults that result in failures. Testing detects the presence of a fault in the software while debugging uses this information to try to find where the fault is.

Basic notions of software testing followed by concepts of regression testing, coverage criteria, and test approaches are presented in this section. 


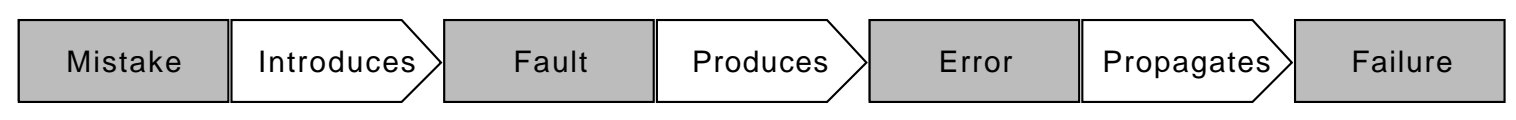

Figure 3 - Testing terms in Software Engineering.

\subsubsection{Faults and Test-cases}

There is some divergence on software testing terms with regard to error, fault, and failure. The IEEE standard 610.12-1990 (IEEE, 1990) provides an explanation of software engineering terms related to the testing activity:

- Mistake: incorrect action performed by a human, which produces incorrect results (e.g., wrong action was taken by the programmer);

- Fault: step, procedure, or incorrect data definition (e.g., incorrect command or instruction);

- Error: difference between expected value and obtained value (e.g., wrong intermediate result of a variable in the software execution);

- Failure: wrong output produced from software execution compared to the expected output (e.g., a wrong result of user-visible events).

Figure 3 shows the relation of testing terms, in which a mistake introduces faults in the software. A fault produces errors that may be not visible, an error is propagated to an output result which may cause a failure. Errors can be classified in domain errors and computational errors. Domain errors are caused by executing an incorrect path (sequence of commands) that is different from the expected path. Computation errors are caused by an incorrect computation but the path executed are the same as the expected path.

A specification is an artifact with an abstract representation of the system created from requirements. A specification is useful to identify failures since the expected results are determined by its analysis. In testing, the act of identifying a failure indicates the presence of a fault in the software. A fault can also be caused by (MCGREGOR, 2001):

- Lack of requirements: when the specification is incomplete due to a missing behavior definition. Requirements inspection can detect such faults;

- System and specification discrepancy: when an implemented functionality does not behave as the specification (a.k.a. functional faults). Testing can detect such faults; 
- Lack of performance, security, scalability or compatibility: when the software execution does not satisfy a non-functional requirement. Requirements analysis and software execution can detect such faults.

In general, a software system (program) has a set of values named input domain, which can be accepted as input. Assume a program $P$ that accepts boolean values, then, the input domain of $P$ is $D=\{$ true, false $\}$.

Definition 2.1.1. A program $P$ with a input domain $D$ is correct for a specification $S$, if the program behaves as the expected behavior of the specification for all input domain values, i.e., $\forall_{d \in D} \bullet S(d)=P(d)$. Given two programs $P_{1}$ and $P_{2}$, if for all $d \in D$ such that $P_{1}(d)=P_{2}(d)$, then, $P_{1}$ and $P_{2}$ are equivalent.

The analysis of a model (specification) can be used to produce a test-case which is an acceptable input event paired with the expected output behavior of the system (MCGREGOR, 2001).

Definition 2.1.2. A test-case (or just a test) is a tuple $(d, S(d))$ such that $d \in D$ is the input and $S(d)$ is the output. A test-suite is a set that contains test-cases.

In general, there are two levels of abstraction for test-cases. Abstract test-cases have abstract input and output values that are executed in the specification, while concrete test-cases are executed in the real program.

In software testing, the test oracle decides whether the expected outputs matches the obtained outputs. The oracle can be a tester, developer, or another program which decides whether a program is correct or not. The testing activity is well succeeded when failures are identified by executing the program using test-cases.

\subsubsection{Test Process and Phases}

In software development, there are several abstraction levels with well-defined phases starting from requirements down to implementation. As said before, testing can be used for the validation of the software that is being built. There are many different process models describing how to relate development and testing phases. For example, the classic $V$-model (IT-BEAUFTRAGTEN, 1997) says that each development phase has a corresponding testing phase. Figure 4 shows the V-model. Starting on the left hand side there are software development phases (top-down) and on the right hand side the testing phases (bottom-up).

Software testing performs a dynamic software analysis divided into four phases/levels (DALAL et al., 1999; AMMANN; OFFUTT, 2008): 


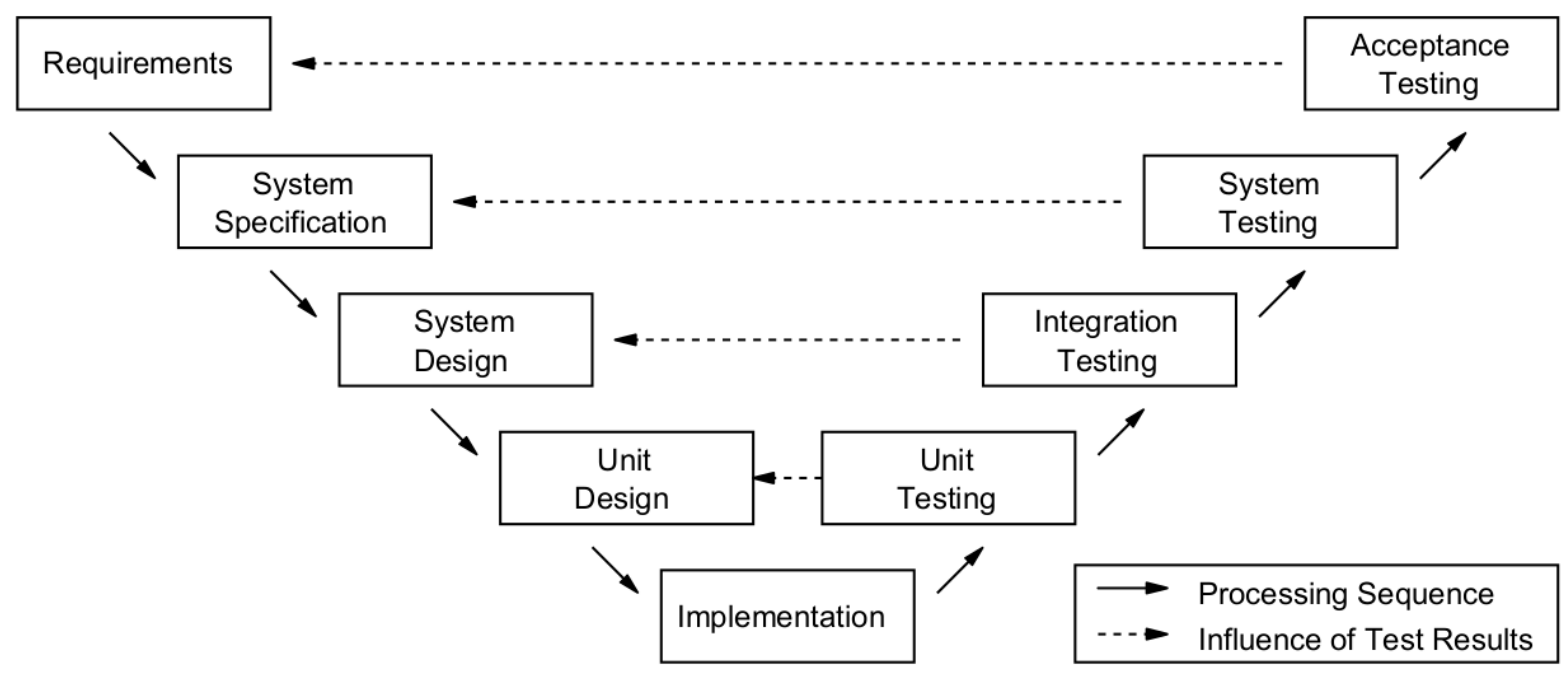

Figure 4 - The V-model (adapted from IT-Beauftragten (1997)).

1. Unit: performs testing of small modules of the software project that may be components, classes, methods, functions, or procedures;

2. Integration: performs testing of module interfaces and their interactions. Incomplete modules may be simulated via drivers and stubs. Drivers emulate module interfaces while stubs simulate the behavior of a module;

3. System: performs testing on the interaction of system in development with other (sub)systems. Implemented functionalities are checked (functional tests) using the software specification which may include the checking of non-functional requirements (non-functional tests);

4. Acceptance: performs testing on the whole system with the client present to check the requirements. Functional and non-functional tests are performed to check every requirement.

There are some test integration approaches that specify the design order of software artifacts in the development process. The classic test integration approach starts by defining requirements followed by software development activities, and finally, tests are designed and executed. Another approach is to perform software development and testing in parallel just after collecting requirements. In agile methods (e.g., eXtreme Programming - XP) test-cases guide the design of functionalities (a.k.a. test-first method). Requirements are collected followed by test design and then software development. The benefit of test-first approaches is project cost reduction and early fault detection within the requirements (AMMANN; OFFUTT, 2008). 
For each test phase, a test process is performed, and software artifacts are generated: test plan, test-cases, data set, software for testing, scripts, and reports. The test process has four steps (MYERS et al., 2004; AMMANN; OFFUTT, 2008):

1. Test planning: a test plan is developed to ease the test process management. A test plan has objectives, criteria used to finish a test phase, schedule, distribution of responsibilities, test-case standard, tool identification, cost estimation, hardware configuration, integration strategy, process monitoring, debugging procedures, and regression testing;

2. Test-case design: test techniques are used to generate test-cases that its execution might result in a failure;

3. Execution: procedures of the test plan;

4. Result evaluation: reports are generated from test results. Expected test results are compared to the real results obtained, and the oracle decides the existence of failures.

In general, once a system is developed, new versions are created further in the maintenance process. New faults may be added after the modifications in the new version. The regression testing verifies whether the system or its components still behave according to the requirements. In addition, regression testing can also be applied to the incremental test development and for each iteration of the process cycle a new version of the system is deployed and tested.

\subsubsection{Regression Testing}

In general, requirement changes or detected faults leads to a new version of the program. Regression testing can ensure that new faults were not introduced in the program by reusing and executing subsets of test-suites from previous versions to test the newest version (YOO; HARMAN, 2012). Let $P$ be a program, $P^{\prime}$ the new version of $P$, and $T$ a test-suite for $P$. Test regression techniques result in a subset of test-cases $T^{\prime} \subseteq T$ to verify whether $P^{\prime}$ still running without new faults. By reducing the size of $T^{\prime}$ that need to be re-executed we can reduce test costs. Regression testing uses three test-case reuse techniques:

1. Selection: test-cases of $T$ that were executed in a previous version $P$ are selected and put into $T^{\prime}$ to test the new version $P^{\prime}$. However, the selection is justified when the cost to select and execute $T^{\prime}$ is smaller than re-executing the whole set $T$. 
2. Prioritization: test-cases of $T^{\prime}$ are ordered for execution according to some criteria. For example, test-cases may be ordered from high to low with regard to the chance of fault detection.

3. Minimization: test-cases of $T^{\prime}$ that are redundant are removed. Test-cases are redundant when they are equivalent to serve a test goal, and they are not required for other test goals. The identification of the best solution is a hard problem.

In general, test selection techniques identify valid test-cases related to the modified parts of the software. A test-case is valid with regard to a specification when the input sequence of the test-case is present (defined) in the specification. For example, a test-case $a \in T$ created from a specification $S$ used to test a program $P$ may not be valid to test a program $P^{\prime}$ based on a specification $S^{\prime}$. In regression testing, test-cases are classified as (YOO; HARMAN, 2012):

- Reusable tests: valid test-cases of a non-modified part of the software that are not re-executed;

- Retestable tests: valid test-cases which must be re-executed that are directly or indirectly related to the changed parts. For example, some test-cases are executed to reach the modified parts;

- Obsolete tests: invalid test-cases from $T$ for a new version of a program $P^{\prime}$.

Regression testing can be corrective or progressive (MAYRHAUSER; ZHANG, 1999). Regression testing is corrective when actions are executed without modifying requirements. In general, corrections are made when non-functional requirements are not satisfied by the specification. For example, performance issues may require another design pattern which does not need new test-cases. Regression testing is progressive when requirements are modified, and new test-cases are required to test modified parts of the software.

Regression testing can be used in the development and maintenance processes (MUCCINI; HOEK, 2003). On maintenance, the testing approach is applied to the new version of the program while in the development a new version of a module can also be tested. Regression testing can be applied to source code and architectural artifacts (BRIAND; LABICHE; HE, 2009). When regression testing is applied to models, the change impact can be analyzed before implementation to allow better planning and cost estimation. When test-cases are traceable to the specification, it is possible to automate the identification of re-testable tests. 


\subsubsection{Coverage Criteria}

Testing the whole program is costly due to a large number of acceptable inputs. Another factor that increases costs is the number of loops in the execution. As a consequence, other means to measure quality are explored. For example, to measure test quality we can use probability or heuristics to support test design and execution (AMMANN; OFFUTT, 2008; DONG et al., 2010).

In software testing, a high level of confidence is required to decide whether a program was well tested. Test coverage criteria is a well-known heuristic for test quality measurement. A test coverage criteria (or just test criteria) decides whether a test-suite $T$ is suitable to test a program $P$. A test criteria $C$ has test requirements and test-case selection methods that select test-cases for $T$. A test-suite $T$ is $C$-suitable to test a program $P$ if $T$ satisfies all requirements of $C$. For example, assume a program $P$ that has a input domain $D=\{$ true, false $\}$ and do not use any other input value. Executing two test-cases $t_{1}=($ true,$a)$ and $t_{2}=($ false,$b)$ of a test-suite $T$ the functional coverage reached is $100 \%$. This level of coverage ensures that $P$ was completely tested regarding the corresponding specification.

Certain levels of test coverage may vary from each organization. In general, critical systems require higher levels of test coverage than common software systems in the market. A low-cost strategy can be elaborated for efficient fault detection using test criteria comparison. For example, consider the study of Mathur and Wong (MATHUR; WONG, 1994) where a program $P$ will be used in a critical security environment. The tester must test $P$ as much as possible, and he/she decides to use several test criteria. First, a simple test criteria $C_{1}$ is selected to generate test-cases. Once all test are performed using $C_{1}$ a better second test criteria $C_{2}$ can be selected to generate test-cases to try to detect more faults. In this scenario, the tester should decide whether the budget will allow the use a third test criteria. Planning the selection and ordering of test criteria is dependent on some comparison factors (WONG; MATHUR, 1995):

- Cost: effort required to design tests for test criteria. It can be measured by the sum of test-cases required to satisfy the criteria or another metric;

- Effectiveness: fault detection capacity of test criteria. In general, test criteria can detect a subset of all possible faults. A test criteria can be compared to another to see which one can detect more faults;

- Strength: is the probability of test criteria to satisfy another. For example, test criteria $C_{1}$ derives test-cases to test a program $P$. How strong a second test criteria $C_{2}$ is to test $P$ in relation to $C_{1}$ ? The answer depends on how many different faults $C_{2}$ is able to detect compared to $C_{1}$. Thus, $C_{2}$ is stronger than $C_{1}$ if $C_{2}$ include $C_{1}$ or $C_{2}$ is complementary to $C_{1}$. 
There is a test criteria hierarchy which determines relations of inclusion and complement (RAPPS; WEYUKER, 1985). One test criteria $C_{1}$ include $C_{2}$ if for any program $P$ and any test-suite $T_{1}$ that is $C_{1}$-suitable, $T_{1}$ is also $C_{2}$-suitable and exists a test-suite $T_{2}$ that is $C_{2}$-suitable but not $C_{1}$-suitable. For example, the specification $S$ of a program $P$ contains a connected graph with nodes and edges. A test criteria $C_{1}$ covers all edges while $C_{2}$ covers all nodes. Test criteria $C_{1}$ include $C_{2}$ because covering all edges include the coverage of all nodes.

\subsubsection{Test Techniques}

Test criteria can be used in the testing of requirement-based specifications down to the implementation. Test techniques may reveal faults during software development using some test criteria. There are three main group of test techniques: functional, structural, and error-based. Such techniques differ based on the kind of information used in the evaluation and generation of test-cases. Test techniques can be classified in (HOWDEN, 1987):

- Specification-based testing (black-box testing): decides whether the program satisfies functional and non-functional requirements. The source code is unavailable and only the functionalities described in a specification are known. The functional group belongs to this category and can be used in procedure/object-oriented contexts and also any test phase;

- Program-based testing (white-box testing): decides whether the source code implementation contains faults and not the specification. Functionalities regarding requirements described in a specification are not evaluated. The structural and error-based groups belong to this category.

These techniques are complementary and can be combined to create an efficient, low-cost test strategy. For example, gray-box test techniques (LINZHANG et al., 2004) combine black-box and white-box techniques where functional test-cases can be designed with access to the source code. In this thesis, we focus on black-box testing.

\section{Functional testing}

The program receives a stimulus event (input) that results in an observable action (output) in response to the stimulus. Thus, only system functions specified from requirements are tested with no concern to the source code (PRESSMAN, 1997). Some functional test criteria are (MYERS et al., 2004):

- Equivalence Class Partitioning: the criteria divides the input domain into classes to generate test-cases. A certain number of test-cases are designed for each equivalence 
class where all inputs inside a class are equivalent. Equivalence classes are classified by valid and invalid. For example, assume that a program accepts integers from 1 to 100. A valid class is an interval including 1 to 100, and an invalid class is the set of integers greater than 100 or smaller than 1 ;

- Boundary Value Analysis: the criteria use boundary values of the program input domain to derive test-cases. For example, assume a program that accepts any integer value. Then, integers have a range from -32768 to +32767 and test-cases use inputs $-32767,-32768$, and -32769 covering the boundary and all close values. Moreover, the criteria can be combined with the equivalence class partitioning;

- Cause and Effect Graph: the criteria use input condition combinations to derive test-cases. The input conditions (cause) and possible actions (effect) are represented by a graph that is converted to a decision table to derive test-cases. For example, assume a program that accepts two parameters: the first an integer; and the second a letter. Several possibilities are tested, the first parameter is a letter instead of an integer, the program receives more than two parameters, etc.

\section{Structural testing}

Structural testing uses the internal structure of the implementation to derive test-cases. Structural testing can be seen as complementary to functional testing, and the resulting information can be used in maintenance, debugging, and software reliability (PRESSMAN, 1997).

In structural testing, the program may be represented by a control flow graph where atomic commands are identified, and execution sequences are grouped in blocks. The nodes of the graph are blocks, and edges are possibilities of a conditional command. A structural criteria derive test-cases from paths of the graph. The basic criteria are (MYERS et al., 2004):

- All-nodes: every node of the graph must be executed at least once. Thus, every command of the program will also be executed at least once;

- All-edges: every edge of the graph must be executed at least once. Thus, every possible condition deviation of the program will also be executed at least once;

- All-paths: every path of the graph must be executed at least once. Thus, every possible combination of command and conditions of the program will also be executed at least once. However, a maximal cycle value is set for loops.

Following the include relation of test criteria all-paths include all-edges and all-edges include all-nodes. 


\section{Error-based testing}

Error-based testing uses information about common mistakes found in the software development process to derive test-cases. Some basic criteria are (MYERS et al., 2004):

- Error seeding: the criteria insert faults in the source code and after test execution faults are identified as natural or artificial. Seeding artificial faults and using a test-suite $T$ for some test criteria $C$ it is possible to say how efficient $T$ is to detect faults. For example, 100 faults are seeded in the source code, and after executing $T$, only 80 faults were detected which means an efficiency of $80 \%$. Natural faults might be detected when executing these tests;

- Mutant analysis: the criteria insert faults in the source code using mutation operators. A mutant is a different version of the code with an artificial fault. Testcases are designed to find specific faults and once a failure is found the mutant is killed. A mutation score measures the level of confidence of test-cases based on generated and killed mutants.

\subsubsection{Test Automation and Quality}

The quality of the testing activity depends on the test criteria used. There is a trade-off between how much to test and how much to spend on testing. In general, there is a large amount of information that must be handled in testing, and at the same time, we need to avoid human mistakes and improve the testing quality. One solution to this problem is to automate the testing activity with the support of tools. Tools can execute costly manual tasks automatically with little effort in a systematic way, thus improving testing productivity. At the same time, it increases testing quality as it avoids human mistakes during the testing activity. One step that is frequently automated in the testing activity is the generation of test-cases for certain test criteria. However, the full automation of the testing activity seems to be impossible due to some undecidable problems, e.g., whether two programs are equivalent to each other. Thus, manual and automatic tasks in the testing activity are seen as complementary.

The automation of the testing activity can reduce application costs, minimize human errors, and ease regression testing (AMMANN; OFFUTT, 2008). Regression testing tools can manage the reuse of test-cases. Tools that implement techniques for impact analysis and test-case traceability reduce costs to generate and compare test results.

The availability of testing tools allows the transfer of technology for industries and contributes to a continuous evolution of such environments, which are essential for developing high-quality software. A testing framework automates the test process, execute 
test-suites, and generate test reports. For example, the JUnit framework (GAMMA; BECK, 2006) can define, integrate and execute unit tests.

\subsection{Model Based Testing}

In software development, a model describes the reality in an abstract and simplified representation for a given purpose (ROTHENBERTG, 1989). A model is formal when it can be manipulated by tools and has a precise, comprehensible, and unambiguous meaning (UTTING; PRETSCHNER; LEGEARD, 2012). Model-Based Testing (MBT) is an approach that uses a formal test model to derive test-cases automatically for functional testing (BROY et al., 2005). A test model is a formal model derived from the requirements which represents the behavior of the software system. A test model can be seen as an abstract representation of a detailed behavioral model created in the development phases that is meaningful to generate significant test-cases.

MBT can be used in any testing phase and some approaches (BASIN; DOSER; LODDERSTEDT, 2006; SCHLäPFER et al., 2009) extend MBT to non-functional tests. Imprecise expectations on the requirements make acceptance testing not being much used in MBT as well. Figure 5 shows the basic application areas of MBT.

Some advantages of model-based testing are (WEISSLEDER, 2009; UTTING; PRETSCHNER; LEGEARD, 2012):

- test models are usually small and easy to understand, verify, and modify;

- test models can provide traceability between requirements and test-cases;

- test models can be used for (semi)-automatic test-case generation given a test coverage criteria;

- testing can be performed before and after the implementation of the system.

\subsubsection{Model-Based Testing Process}

The MBT process uses several test artifacts from requirements for the system under test. A System Under Test (SUT) is composed of the code implementation and the required infrastructure to run it. Figure 6 shows an overview on the relation between MBT artifacts with solid arrows. First, the requirements are captured in the top-left corner. Then, from requirements, the development model, test model and test-case specifications (based on a test coverage criteria) are generated. From both test model and test-case specifications, test-cases are created/reused, concretized/selected and then executed in the SUT. Finally, an oracle compares the results which generate a verdict. Dashed arrows between development model and test model represent the possibility of generating test 


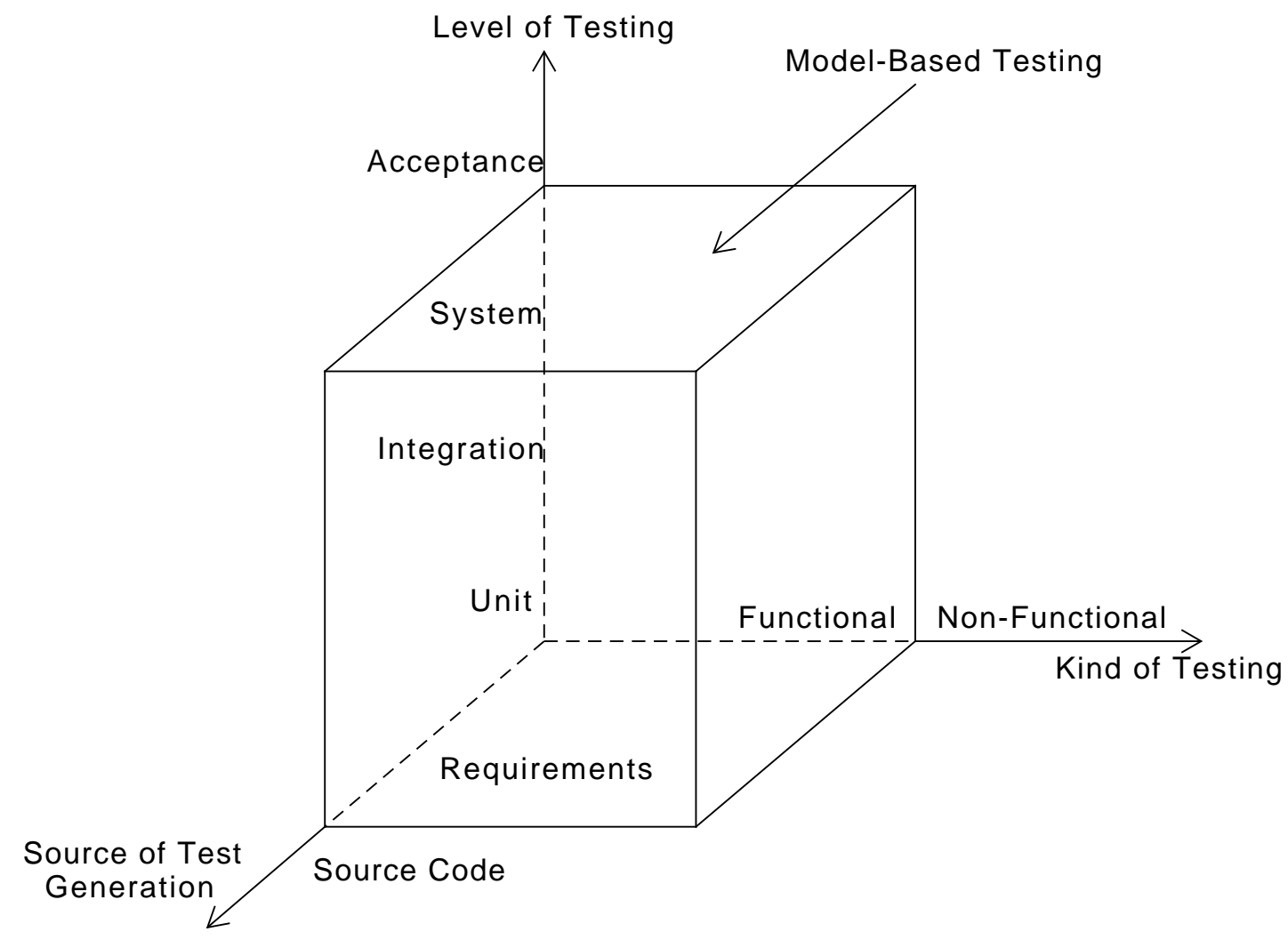

Figure 5 - Application fields of model-based testing (adapted from Weissleder (2009)).

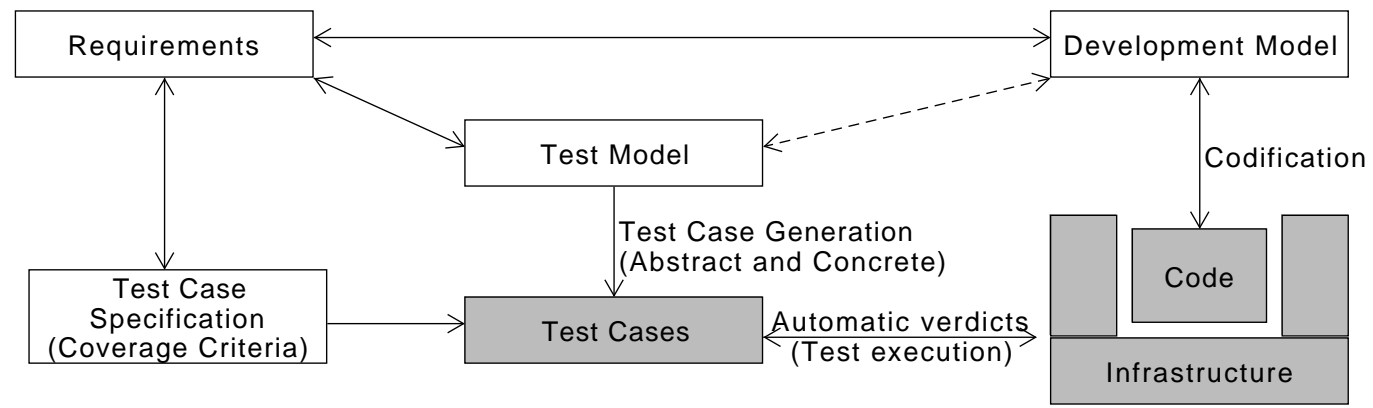

Figure 6 - Model-based testing overview (adapted from Pretschner and Philipps (2004)).

models from development models using, e.g., model transformation techniques (LLANA, 2012).

The MBT process has seven steps (PRETSCHNER; PHILIPPS, 2004; UTTING; PRETSCHNER; LEGEARD, 2012):

1. Requirement understanding: the tester needs to understand how the software works in a given environment following some guidelines:

a) Identify characteristics or components to be tested; 
b) Create communication channels between development groups to allow reuse and model adaptation;

c) Enumerate inputs and outputs for automation;

d) Identify input sequences that need to be modeled to ease model design;

e) Constantly update the requirement model for a better understanding.

2. Modeling: the tester creates a test model to represent the behavior of a component, subsystems, or the whole SUT. In general, the test model is an abstract representation of the SUT;

3. Test criteria definition: the tester needs to select suitable test criteria and a tool to support test-case generation;

4. Test-case generation: the tester needs to select a method for automatic test-case generation using the test model and test criteria;

5. Test-case concretization: the tester needs to transform (concretize) abstract testcases in executable test-cases for the SUT. The test model and the SUT are at different abstraction levels. Thus, to concretize a test-case, the tester can transform test-cases into test scripts, design an adapter or use both scripts and an adapter. An adapter can translate inputs and outputs between a test model and an SUT using a concretization and an abstract function, respectively;

6. Test execution: the tester needs to execute concrete test-cases in the SUT using the off-line or on-line approach. The off-line approach separates test-case generation from test-case execution. First, all test-cases are generated and then they are executed in sequence for an easy test management. The on-line approach generates test-cases and executes them dynamically (on-the-fly) where the next test-case generated is dependent on the result of the previous test-case;

7. Result analysis: the oracle checks the results provided from the test-cases execution to generate verdicts. An oracle is a tester or a program that can automatically compare the abstract expected output from the test model specification to the real output provided from the SUT. A verdict is the result of a test-case execution that may pass, fail, or be inconclusive. A test-case pass when the execution succeeds and there is no failure. A test-case fails when the execution succeeds, and a failure is found. A test-case is inconclusive when the execution ends early, but no failure is found.

\subsubsection{Test-case Concretization}

One important step in the MBT test process is the concretization of abstract test-cases (UTTING; LEGEARD, 2006). Generated abstract tests are augmented with 
concrete implementation-specific data making them executable. Checking the conformance of a software system requires extra effort for each new test that needs to be concretized. According to case studies (UTTING; LEGEARD, 2006; Graham D., 2012), the cost to manually concretize a test-case is several times (around 200) greater than the cost of executing the same concretized test. To tackle this problem, adapters can be developed to automate the concretization process. However, the adapters often need to be modified for new versions/products. For example, systems that constantly evolve (e.g., graphical user interface systems) cannot afford to update adapters of each new version of the system, which often take more time than manually testing the system in the first place (DEV; JÄÄSKELÄINEN; M., 2012).

\subsubsection{Model-Based Testing Techniques}

In this section, we present some techniques that are used in MBT approaches. In this thesis, we present algorithms that were inspired by some MBT techniques as graph search algorithms, constraint solving, model checking, abstract interpretation, and model slicing.

\section{Graph-Search Algorithms}

In general, behavioral models are represented by specifications based on graphs with nodes and edges. For example, state machines are based on graphs with states (nodes) and transitions (edges). The relation between those elements built paths that describe the behavior of the system. Graph-search algorithms are useful to find paths with certain properties in the model. For example, to check a specific behavior of the system, we may use graph-search algorithms to find the shortest path to reach a specific state (PAP et al., 2007).

\section{Constraint Solving}

The Constraint Satisfaction Problem (CSP) is defined by a set of objects which need to satisfy a set of constraints. Logic puzzle problems can be modeled as a CSP, e.g., crosswords and sudoku. To solve the problem, we need to find one valid set of objects that is known as constraint solving. In general, some approaches for constraint solving are specific to a domain application. Solvers for Satisfiability (SAT Solvers) are often used for hardware verification but can be used to represent the domain extensibility, e.g., in software product lines (CZARNECKI; PIETROSZEK, 2006). Most of these solvers have tool support for constraint solving. In this thesis, we use the SAT solver of the Z3 tool (MOURA; BJØRNER, 2008). 


\section{Model Checking}

Specifications based on state machines might have some properties (e.g., the presence of deadlocks) that require verification. The model checking approach can automatically verify such properties. First, it negates the property and checks whether the model does not meet the negated property, thus, returning a counter-example. Test design approaches can also use model checking for automatic test generation (CLASSEN et al., 2011; CLASSEN et al., 2013). However, in this thesis, we use the model checking concepts to validate some model properties. A survey of testing with model checkers can be found in (FRASER; WOTAWA; AMMANN, 2009).

\section{Abstract Interpretation}

The abstract interpretation is a technique that can deduce information about the system without executing it and keeping only some relevant abstract information to describe the semantics of the system. An abstraction function creates an abstraction of the real system while a concretization function transforms these properties back to the real system. Besides, abstract interpretation can also be used for testing (PRENNINGER; PRETSCHNER, 2005; BLECH; MOU; RATIU, 2012).

\section{Model Slicing}

Slicing is a technique that simplifies the management of programs or models by removing unnecessary parts. Structural and behavioral artifacts can be selected (sliced) to focus on a specific part (slice) for better understanding and ease test validation and generation (KAMISCHKE; LOCHAU; BALLER, 2012).

\subsubsection{Test-case Generation for Finite State Machines}

The test model must be an abstract version of the development model or at least easier to verify, modify and maintain. However, the test model must be precise to derive significant test-cases. There is no suitable test model for every purpose. Thus, modeling techniques can be selected to reach a certain objective. Some formal models used in MBT are: Finite State Machines, Extended Finite State Machines, Labeled Transition Systems, and Input/Output Transition Systems (UTTING; PRETSCHNER; LEGEARD, 2012; OSTER et al., 2012b). Although testing based on state machines has been explored in the last decades, however, there are some challenges to consider. In this thesis, we investigate the generation of configurable test-suites using test models based on state machines by extending some of their formalisms and methods to software product lines. 
In this section, we start presenting the finite state machine formalism, followed by state, transition, and full fault coverage criteria, and finally describing related test-case generation methods.

\section{Finite State Machines}

The classic Finite State Machine (FSM) formalism is often used due to its simplicity and rigor for systems such as communication protocols and reactive systems (BROY et al., 2005).

Definition 2.2.1. An FSM $M$ is defined by a 5 -tuple $\left(S, s_{0}, I, O, T\right)$, where $S$ is a finite set of states, $s_{0} \in S$ is the initial state, $I$ is the set of inputs, $O$ is the set of outputs, and $T$ is the set of transitions in the form of $t=\left(s, x, o, s^{\prime}\right) \in T$, where $s \in S$ is the source state, $x \in I$ is the input label, $o \in O$ is the output label, and $s^{\prime} \in S$ is the target state.

The FSM model can be used as a graph-based test model for test design where paths are selected to be executed. Moreover, paths are essential to define some FSM properties due to the tuple definition that binds FSM elements.

Definition 2.2.2. Given an input sequence $\alpha=\left(x_{1}, \ldots, x_{k}\right), x_{i} \in I, 1 \leq i \leq k$, a path from state $s_{1}$ to $s_{k+1}$ exists when there are transitions $t_{i}=\left(s_{i}, x_{i}, o_{i}, s_{i+1}\right) \in T$, for each $1 \leq i \leq k$. A path $v$ is a 3 -tuple $(\tau, \alpha, \beta)$, where

1. $\tau=\left(s_{1}, \ldots, s_{k+1}\right) \in S^{*}$ is the state sequence,

2. $\alpha=\left(x_{1}, \ldots, x_{k}\right) \in I^{*}$ is the input sequence,

3. $\beta=\left(o_{1}, \ldots, o_{k}\right) \in O^{*}$ is the output result.

Notation $\Omega(s)$ is used to denote all paths that start on state $s \in S$ and $\Omega_{M}$ is used to denote $\Omega\left(s_{0}\right)$. Given a path $\left(\left(s_{0}, \ldots, s\right), \alpha, \beta\right) \in \Omega_{M}, s$ can be reached using the input sequence $\alpha$.

\section{Validation Properties}

Methods that generate test-cases from FSMs usually require FSMs to possess some of the properties defined below.

Definition 2.2.3. The following validation properties are defined for FSMs:

1. Deterministic: if two transitions leave a state with a common input, then both transitions reach the same state:

$$
\forall\left(s, x, o, s^{\prime}\right),\left(s, x, o^{\prime}, s^{\prime \prime}\right) \in T \bullet s^{\prime}=s^{\prime \prime}
$$




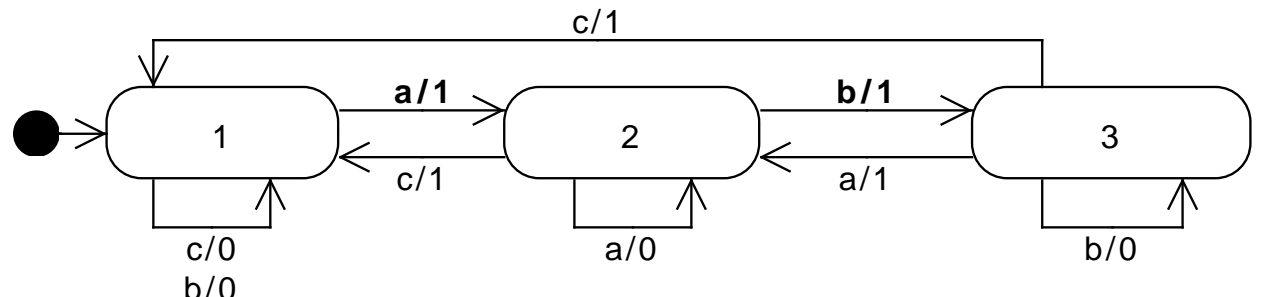

Figure 7 - Abstract FSM $M$.

2. Complete: every state has one transition for each input:

$$
\forall_{s \in S, x \in I} \bullet \exists_{o \in O, s^{\prime} \in S} \bullet\left(s, x, o, s^{\prime}\right) \in T
$$

3. Initially Connected: there is a path to every state from the initial state:

$$
\forall_{s \in S} \bullet \exists_{\alpha \in I^{*}, \tau \in T^{*}, \beta \in O^{*}} \bullet\left(\left(s_{0}, \ldots, s\right), \alpha, \beta\right) \in \Omega_{M}
$$

4. Minimal: all pairs of states are distinguishable:

$$
\forall_{s_{a}, s_{b} \in S} \bullet \exists_{\left(\left(s_{a}, \ldots, s_{a^{\prime}}\right), \alpha, \beta_{a}\right) \in \Omega\left(s_{a}\right),\left(\left(s_{b}, \ldots, s_{b^{\prime}}\right), \alpha, \beta_{b}\right) \in \Omega\left(s_{b}\right)} \bullet \beta_{a} \neq \beta_{b}
$$

Example 1. The Figure 7 presents a deterministic, complete, initially connected, and minimal FSM $M=\left(S, s_{0}, I, O, T\right)$, where $S=\{1,2,3\}, s_{0}=1, I=\{a, b, c\}, O=\{0,1\}$, and $T=\{(1, a, 1,2),(1, b, 0,1),(1, c, 0,1),(2, a, 0,2),(2, b, 1,3),(2, c, 1,1),(3, a, 1,2)$, $(3, b, 0,3),(3, c, 1,1)\}$. By default, the initial state $s_{0}$ is reached by the empty input sequence $\varepsilon$. Two paths contain the transitions $(1, a, 1,2)$ and $(2, b, 1,3)$ (highlighted) that are used to reach states 2 and 3 .

\section{Test-cases}

To detect the presence of faults in conformance testing, test-cases are used to verify the implemented behavior.

Definition 2.2.4. Given an FSM $M=\left(S, s_{0}, I, O, T\right)$, an input sequence $\alpha \in I^{*}$ is defined for $M$ on state $s_{0}$ when there is a path $\left(\left(s_{0}, \ldots, s\right), \alpha, \beta\right) \in \Omega_{M}$ (Definition 2.2.2) where $\alpha$ reaches $s$. A test-case (input part) of $M$ is a defined input sequence $\alpha \in I^{*}$ and the prefix-closed set (Definition 2.2.6) of test-cases of $M$ is called a test-suite of $M$.

Next, we present preliminaries to define a prefix-closed set of test-cases.

Definition 2.2.5. Given input sequences $\alpha, \beta, \gamma \in I^{*}$, an input sequence $\alpha$ is the prefix of an input sequence $\beta$ when $\beta=\alpha \gamma$ for the input sequence $\gamma$, and $\gamma$ is a suffix of $\beta$. An 
input sequence $\alpha$ is a proper prefix of $\beta$ when $\beta=\alpha \omega$ for some $\omega \neq \varepsilon$, where $\varepsilon$ is the empty sequence. Moreover, we say that a sequence $\alpha \in A$ is maximal in $A$ if there is no sequence $\beta \in A$ such that $\alpha$ is a proper prefix of $\beta$.

Definition 2.2.6. Given a set of input sequences $A \in \mathcal{P}\left(I^{*}\right)$ and an input sequence $\beta \in A$, the set of prefixes of $\beta$ is denoted by $\operatorname{pref}(\beta)$ i.e. $\operatorname{pref}(\beta)=\{\alpha \mid \beta=\alpha \omega\}$. Similarly, $\operatorname{pref}(A)$ is the set of all prefixes of all input sequences $\beta \in A$, i.e., $\bigcup_{\beta \in A} \operatorname{pref}(\beta)$. When $A=\operatorname{pref}(A)$ the set $A$ is called prefix-closed.

\section{State Coverage Criteria}

The state coverage criteria require defined input sequences that can reach states. We assume FSMs that are deterministic and initially connected. A test-suite TS satisfies the state coverage criteria when it contains tests that can reach all states of the FSM.

Definition 2.2.7. Given an FSM $M=\left(S, s_{0}, I, O, T\right)$ and a state $s \in S$, the test-suite $T S \subseteq \mathcal{P}\left(I^{*}\right)$ covers $s$ if there exists a path $\left(\left(s_{0}, \ldots, s\right), \alpha, \beta\right) \in \Omega_{M}$ to reach $s$ such that $\alpha \in T S$. The test-suite $T S$ is a state cover set (for $M$ ) if it covers every state of $M$ :

$$
\forall_{s \in S} \bullet \exists_{\left(\left(s_{0}, \ldots, s\right), \alpha, \beta\right) \in \Omega_{M}} \bullet \alpha \in T S
$$

Example 2. Following the state coverage criteria, the set $T S=\operatorname{pre} f(\{a b\})$ is a state cover set for the FSM $M$ presented in Figure 7.

\section{Transition Coverage Criteria}

The transition coverage criteria require defined input sequences that can reach the source state of a transition and including its input. A test-suite TS attends the transition coverage criteria when it contains tests that can reach all transitions of the FSM.

Definition 2.2.8. Given an FSM $M=\left(S, s_{0}, I, O, T\right)$, and a state cover set $T S \subseteq$ $\mathcal{P}\left(I^{*}\right)$ for $M$, the test-suite $T S$ covers a transition $\left(s, x, o, s^{\prime}\right) \in T$ if there exists a path $\left(\left(s_{0}, \ldots, s\right), \alpha, \beta\right) \in \Omega_{M}$ from state $s_{0}$ to $s$, where $\alpha \in T S$ is an input sequence to reach $s, \beta$ is the output sequence, and $\alpha x \in T S$. The set $T S$ is a transition cover test-suite of $M$ if it covers every transition of $M$ :

$$
\forall_{\left(s, x, o, s^{\prime}\right) \in T} \bullet \exists_{\alpha \in T S} \bullet \exists_{\left(\left(s_{0}, \ldots, s\right), \alpha, \beta\right) \in \Omega_{M}} \bullet \alpha x \in T S
$$

We used a breadth-first search algorithm to find all transitions it results in a testing tree. 


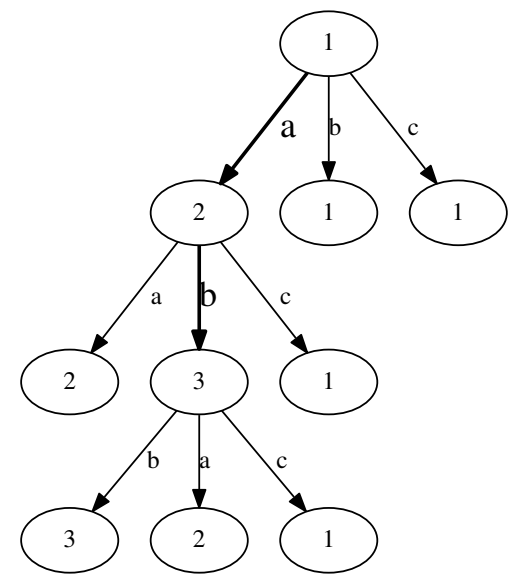

Figure 8 - Testing tree of a transition cover set.

Example 3. Figure 8 presents a testing tree generated by the transition cover set $T S=$ $\operatorname{pref}(\{b, c, a c, a a, a b a, a b b, a b c\})$ for $M$ of Figure 7 . Note that the transition cover set extends the state cover set.

\section{Full Fault Coverage Criteria}

To define the full fault coverage criteria, we use sufficiency conditions based on convergence and divergence properties based on a fault domain.

Definition 2.2.9. Given an FSM $M=\left(S, s_{0}, I, O, T\right)$, two test-cases (Definition 2.2.4) $\alpha$ and $\beta$ of $M$ are convergent when both test-cases reach the same state, and they are divergent when they reach different states.

The convergence and divergence are extended to sets of FSMs defined as a fault domain.

Definition 2.2.10. Assume an FSM $M=\left(S, s_{0}, I, O, T\right)$ with $n$ states and its implementation $N=\left(Q, q_{0}, I, O^{\prime}, T^{\prime}\right)$. The symbol $\Im$ denotes the fault domain that is the set of all FSMs that are: (i) deterministic (Definition 2.2.3 item 1); (ii) have the same input alphabet as $M$; (iii) and include all defined input sequences (Definition 2.2.4) of $M$ (i.e., $\left.\forall_{N \in \Im} \bullet \forall_{\left(\left(s_{0}, \ldots, s\right), \alpha, \beta\right) \in \Omega_{M}} \bullet \exists_{\left(\left(q_{0}, \ldots, q\right), \alpha^{\prime}, \beta^{\prime}\right) \in \Omega_{N}} \bullet \alpha=\alpha^{\prime}\right)$. Moreover, $\Im_{n}$ is the set of FSMs from $\Im$ with $n$ states.

Distinguishing two FSMs uses input sequences that are applied to their initial states.

Definition 2.2.11. Given a test-suite $T, k(k \geq 2)$ FSMs are $T$-equivalent when all testcases of $T$ applied to all $k$ FSMs return the same output sequence. The subset $\Im_{n}(T) \subseteq \Im_{n}$ 
denotes all FSMs of $\Im_{n}$ which are $T$-equivalent to $M$. Moreover, given two test-cases $\alpha, \beta \in T$ they are $T$-separated when there are test-cases $\alpha \gamma, \beta \gamma \in T$ that return different output sequences.

Thus, $T$-separated test-cases diverge in every FSM that is $T$-equivalent to $M$.

Lemma 1 (Simao and Petrenko (2010)). Given a test-suite $T$ of an FSM $M, T$-separated tests are $\Im(T)$-divergent.

We refer to Simao and Petrenko (2010) for detailed proofs of the results presented in this section.

Lemma 2 (Simao and Petrenko (2010)). Given a test-suite $T$ and $\alpha \in T$, let $K$ be an $\Im_{n}(T)$-divergent set with $n$ tests and $\beta \in K$ be a test $M$-convergent with $\alpha$. If $\alpha$ is $\Im_{n}(T)$-divergent with each test in $K \backslash\{\beta\}$, then $\alpha$ and $\beta$ are $\Im_{n}(T)$-convergent.

To define the completeness of a test-suite $T$ is used the notion of preserving convergence between $M$ and FSMs of $\Im_{n}$.

Definition 2.2.12 (Simao and Petrenko (2010)). Given a test-suite $T$ of an FSM $M$, a set of tests is $\Im_{n}(T)$-convergence-preserving (or, simply, convergence-preserving) if all its $M$-convergent tests are $\Im_{n}(T)$-convergent.

The following theorem summarizes the main results from Simao and Petrenko (2010) where the full fault coverage criteria is established based on convergence and divergence properties.

Theorem 1 (Simao and Petrenko (2010)). Given a test-suite $T$ for an FSM $M$ with $n$ states, $T$ is $n$-complete when for all FSMs $N \in \Im_{n}$ there exist tests in $T$ that distinguish $M$ and $N$ (Definition 2.2.11). If $T$ has an $\Im_{n}(T)$-convergence-preserving transition cover set for $M$ that includes the empty symbol $\varepsilon$ (initialized), then $T$ is an $n$-complete test-suite for $M$.

A test-suite $T$ satisfies the full fault coverage criteria when it contains an $n$-complete for an FSM $M$, then, by executing $T$ we are capable of detecting any fault in all FSM implementations $N \in \Im_{n}(T)$.

\section{Test-case Generation Methods}

In the Model-Based Testing (MBT) approach we select a test criteria to design test-cases using a behavioral test model. The resulting test-suite execution must be able to detect as much faults as possible. Thus, some test criteria have the right balance between 
test costs and high fault detection. The full fault coverage is one of test criteria with such balance.

In general, test-case generation has several limitations related to the testing activity. Thus, most automatic test-case generation proposals use heuristics to find good test-suites since the best solution is a hard problem. There exist several methods to generate $n$ complete test-suites (CHOW, 1978; LUO et al., 1994; SIMAO; PETRENKO, 2010) for the full fault coverage criteria. For example, the incremental P method (SIMAO; PETRENKO, 2010) uses two input parameters: a deterministic, initially connected, and minimal FSM $M$; and an initial test-suite $T$. The initial set $T$ can be empty, and new test-cases are added/incremented (if necessary) until an $n$-complete test-suite for $M$ is produced. Therefore, the $\mathrm{P}$ method checks if all implementations $N \in \Im_{n}$ can be distinguished from $M$ using $T$, and decides if more sequences need to be added to $T$. Experimental evaluation indicates that the $\mathrm{P}$ method often results in smaller $n$-complete test-suites compared with other methods (ENDO; SIMAO, 2013).

In this thesis, we investigate the HSI and $\mathrm{P}$ methods to generate test-cases for the full fault coverage criteria in two directions of research explained in the contributions section. We refer to Simao and Petrenko (2010) for a detailed explanation of the P method. Next, we introduce the basic notions of the HSI method explained in (LUO et al., 1994).

The HSI method was based on the W method (CHOW, 1978) where they use a characterizing set to select Harmonized State Identifiers (HSI) sets to distinguish pairs of states in the FSM.

Definition 2.2.13. Given an $\operatorname{FSM} M=\left(S, s_{0}, I, O, T\right)$ with state set $S=\left\{s_{1}, \ldots, s_{n}\right\}$, the set $W \subseteq \mathcal{P}\left(I^{*}\right)$ is a characterizing set if and only if for all $1 \leq i, j \leq n$ with $i \neq j$ there exists an input sequence (separating sequence) $\gamma \in W$ that distinguishes $s_{i}$ and $s_{j}$ :

$$
\forall_{s_{i}, s_{j} \in S} \bullet \exists_{\left(\left(s_{i}, \ldots, s_{i^{\prime}}\right), \gamma, \beta_{i}\right) \in \Omega\left(s_{i}\right),\left(\left(s_{j}, \ldots, s_{j^{\prime}}\right), \gamma, \beta_{j}\right) \in \Omega\left(s_{j}\right)} \bullet \beta_{i} \neq \beta_{j}
$$

The sets $H_{1}, \ldots, H_{n} \subseteq \mathcal{P}\left(I^{*}\right)$ are harmonized state identifiers if and only if for all $1 \leq i, j \leq n$ with $i \neq j$ there exist subsets $H_{i} \subseteq W$ and $H_{j} \subseteq W$ for $s_{i}$ and $s_{j}$ such that a common prefix $\gamma$ of $\gamma_{i} \in H_{i}$ and $\gamma_{j} \in H_{j}$ distinguishes $s_{i}$ and $s_{j}$.

To generate test-cases the HSI method concatenates a transition cover set with HSI sets and return the final test-suite.

Definition 2.2.14. Given a transition cover set $C V$ for $M$ and harmonized state identifiers sets $H_{i}$, the HSI method returns a test-suite $T S$ by concatenating $C V$ with every $H_{i}$ set for each $s_{i} \in S$ such that only tests of $C V$ that reach $s_{i}$ are concatenated:

$$
\forall_{s_{i} \in S} \bullet \forall_{\alpha \in C V} \bullet \exists_{\left(\left(s_{0}, \ldots, s_{i}\right), \alpha, \beta\right) \in \Omega_{M}} \bullet \forall_{h \in H_{i}} \bullet \alpha h \in T S
$$


Example 4. The characterizing set $W$ for FSM $M$ presented in Figure 7 is $W=\{a, c\}$, while the HSI sets are: $H_{1}=\{a, c\}, H_{2}=\{a\}$, and $H_{3}=\{a, c\}$. The complete test-suite is obtained by concatenating $C V=\operatorname{pre} f(\{b, c, a c, a a, a b a, a b b, a b c\})$ with $H_{i}$ sets, which results in $T S=\operatorname{pref}(\{b a, b c, c a, c c, a c a, a c c, a a a, a b a a, a b b a, a b b c, a b c a, a b c c\})$.

\subsection{Software Product Lines}

Software design has evolved, and new requirements for custom/extensible software emerged while the expected release time was reduced. To satisfy such necessities new approaches in the software engineering appeared. The Software Product Line Engineering (SPLE) approach does systematic reuse of core assets represented by software artifacts to instantiate, generate or assemble multiple similar systems that form a software product line (CLEMENTS; NORTHROP, 2001).

A Software Product Line (SPL) is a set of software programs that share features to satisfy a specific domain (CLEMENTS; NORTHROP, 2001). An SPL is derived from a reusable software architecture resulting in a product family. Products can vary regarding behavior, quality attributes, platform, physical configuration and middleware (LINDEN; SCHMIF; ROMMES, 2007). The SPL software architecture defines requirements, components and processes that are shared, reused and managed by commonalities and variabilities. Commonalities manage product similarities while variabilities manage product differences using features. Features are functional or non-functional requirements that may be classified into three types (LINDEN; SCHMIF; ROMMES, 2007):

- Common: this feature describe a characteristic that is present in every derivable product;

- Variable: this feature describes a characteristic that is present in only some products but not all of them. If there is a module that implements this feature, then, it must be reusable;

- Product-specific: this feature describes a characteristic that may be present in only one product. The client may ask for a new specific feature, and the SPL architecture must be able to support it.

For example, assume an SPL for mobile phones. A common feature can be a fixed communication module that is present in every cell phone. A variable feature can be a video camera provided by different brands where some models use a specific camera. A product-specific feature can be a TV module which was not initially considered in the SPL. 
Developing an SPL from scratch requires more effort than developing a single software system. However, the SPL infrastructure allows the systematic derivation of several products to increase productivity, reduce development costs, and ease product evolution. In general, the extra effort to develop an SPL is compensated after deriving the third product (LINDEN; SCHMIF; ROMMES, 2007).

\subsubsection{Development Process}

The SPL development process performs a systematic reuse on requirements, architecture artifacts, components, and tests to develop other products. The SPL development process is separated into two levels:

- Domain engineering (platform development): development of common and reusable components;

- Application engineering: development of products by instantiating/assemble reusable components.

The domain engineering level has four steps (POHL; BÖCKLE; LINDEN, 2005):

1. Domain analysis: analyze domain characteristics and represent features in a feature model.

2. Core asset development: design, implement, test common (core) and reusable artifacts, then, store them in a repository. In general, these artifacts contain features that are mapped to model elements.

3. Production plan: elaborate guidelines to derive individual products from core artifacts which may include model transformation, code generation, and compilation.

4. Product management: create maintenance routines that describe methods and strategies to manage variability. For example, a common feature may be updated and turn into a variable feature.

The application engineering level has three steps (POHL; BÖCKLE; LINDEN, 2005):

1. Product characterization: select features from a feature model to characterize the product that is going to be built.

2. Product synthesis: retrieve reusable artifacts from the repository which are required to build the product.

3. Product construction: process reusable artifacts following the production plan until the final product is built. 


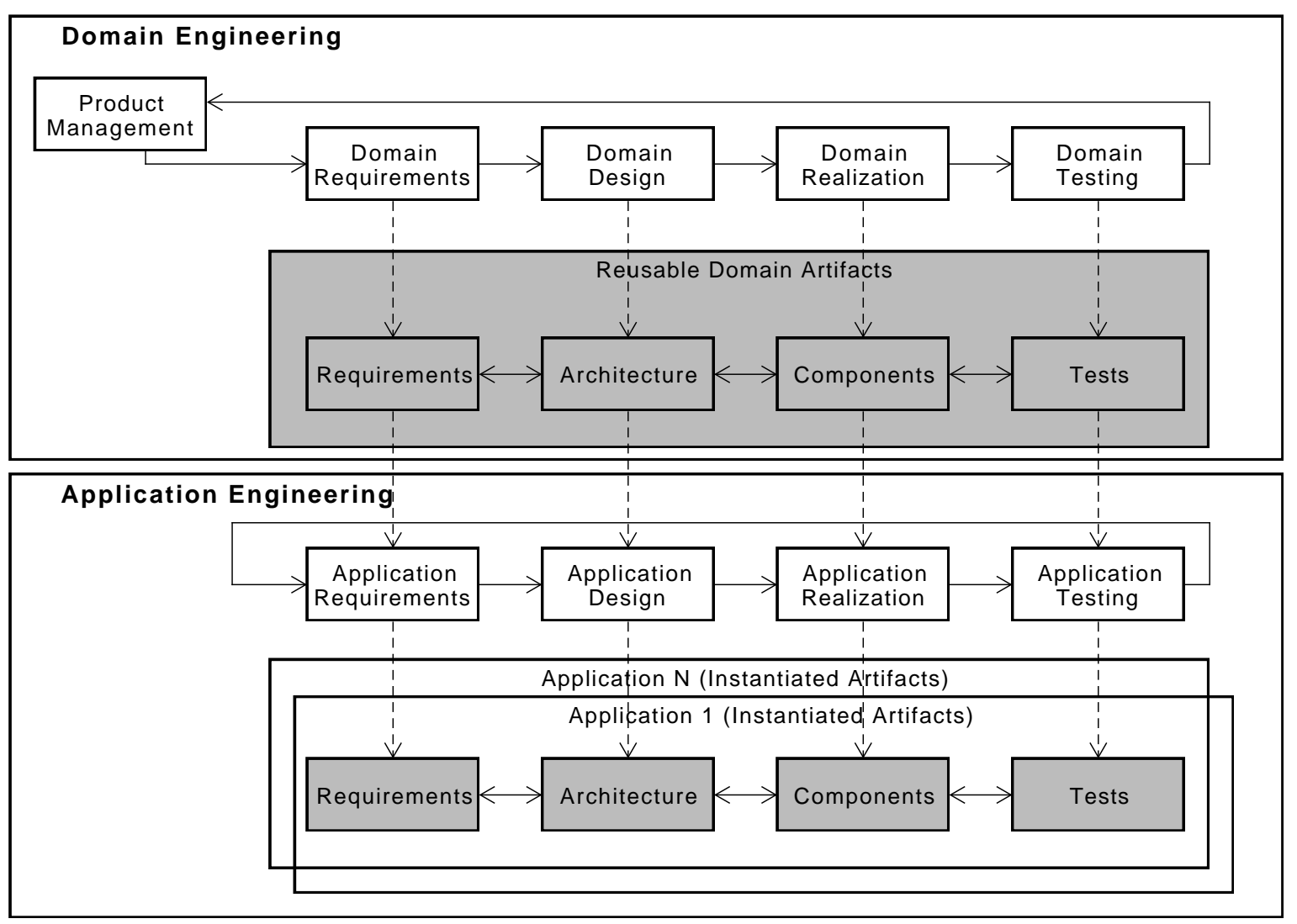

Figure 9 - Software product line development process (adapted from Pohl, Böckle and Linden (2005)).

Figure 9 presents an overview of the whole SPL process and related artifacts. Software artifacts developed at the domain engineering are reused in the application engineering. Dashed arrows illustrate the flow of software artifacts while normal arrows illustrate the process cycles. The resulting application is derived using artifacts with common, variable and product-specific parts.

\subsubsection{Feature Model}

A feature is a prominent or distinctive user-visible aspect, quality, or characteristic of a software system or system (KANG et al., 1990). Feature models (POHL; BÖCKLE; LINDEN, 2005) define feature relations from commonalities and variabilities using graphical models such as feature diagrams. A feature diagram (SCHOBBENS; HEYMANS; TRIGAUX, 2006) uses a notational convention to describe constraint-based feature relations. The basic feature relations are mandatory, optional, inclusive-OR (or), exclusive-OR (alternative), include and exclude (KANG et al., 2007). A noteworthy feature modeling method was the Feature-Oriented Domain Analysis (FODA) (KANG et al., 1990). Most subsequent feature modeling methods extended the FODA to add new dependency relations such as the Orthogonal Variability Model (OVM) (LINDEN; SCHMIF; ROMMES, 


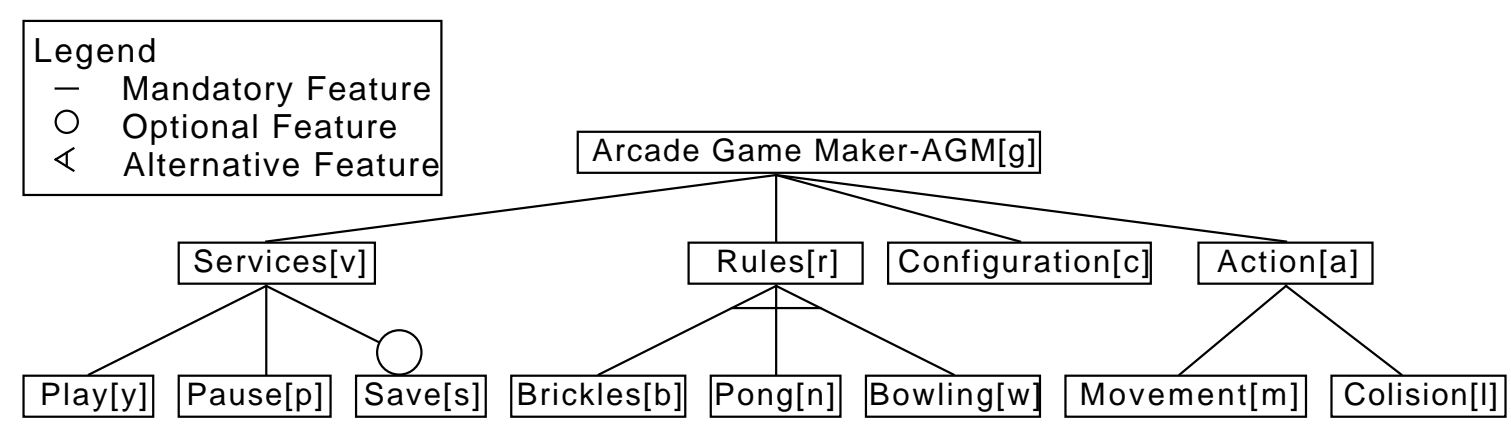

Figure 10 - AGM Feature Model (adapted from SEI (2011)).

2007).

Example 5. The Arcade Game Maker (AGM) (SEI, 2011) can produce arcade games with different game rules. Figure 10 shows the feature diagram of AGM based on FODA. There are three alternative features for the game rule (Brickles, Pong, and Bowling) and one optional feature (Save) to save the game. One and only one alternative feature must be selected, and the optional feature is left open for selection.

A feature diagram is developed in the domain engineering and used as input to the application engineering level where it is instantiated by a configuration model. A configuration model allows the selection of features to specify a single product, and it is useful to recover and integrate components for the product configuration process. The product configuration process (binding) derives a specific product using the reusable SPL architecture and a configuration model with selected features.

In general, due to the dependencies and constraints on feature combinations, only some products can be derived. Assume a set of features $F$ of a feature model. The set of all valid products $P$ of an SPL is a subset of feature combinations from the power set $\mathcal{P}(F)$ that satisfies the constraints specified by the feature model (BATORY, 2005).

A feature constraint $\chi$ is a propositional formula that interprets elements of the feature set $F$ as propositional variables. The set of all feature constraints is denoted by $B(F)$. Feature relation and constraints of a feature diagram can be extracted as a feature constraint following a formal semantics (SCHOBBENS; HEYMANS; TRIGAUX, 2006). A product configuration $\rho \in B(F)$ of a product $p \in P$ is a feature constraint of the form $\rho=\left(\bigwedge_{f \in p} f\right) \wedge\left(\bigwedge_{f \notin p} \neg f\right)$, i.e., the conjunction of all features present in $\rho$ and the conjunction of all features absent from $\rho$. The set $\Lambda \subseteq B(F)$ denotes all valid product configurations of the SPL. Given a feature constraint $\chi \in B(F)$, a product configuration $\rho \in \Lambda$ satisfies $\chi$ (denoted by $\rho \vDash \chi$ ), if and only if the feature constraint $\rho \wedge \chi$ is satisfiable. 


\begin{tabular}{|c|c|c|}
\hline \multicolumn{3}{|c|}{ Product Configurations } \\
\hline \hline Product 1 & Product 3 & Product 5 \\
B\&!N\&!W\&!S & !B\&N\&!W\&!S & !B\&!N\&W\&!S \\
\hline Product 2 & Product 4 & Product 6 \\
B\&!N\&!W\&S & !B\&N\&!W\&S & !B\&!N\&W\&S \\
\hline
\end{tabular}

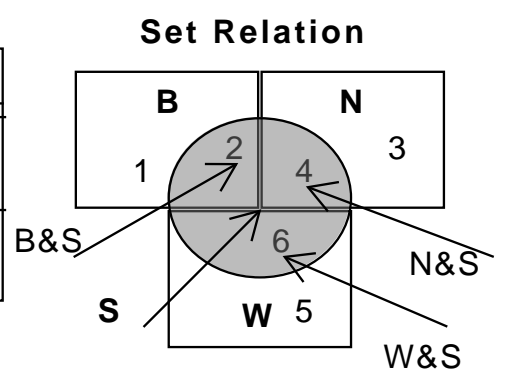

Figure 11 - Satisfiable product configurations of AGM.

Example 6. Given the feature diagram of Figure 10 the extracted feature set is $F=$ $\{G, V, R, C, A, Y, P, S, B, N, W, M, L\}$ where $O=\{G, V, R, C, A, Y, P, M, L\} \subseteq F$ is the subset of mandatory features. The extracted feature constraint that represent the relation of all features is:

$$
\begin{aligned}
& \chi=\left(\left(\bigwedge_{f \in O} f\right) \wedge(S \Longrightarrow V) \wedge(B \vee N \vee W) \wedge\right. \\
& \neg(B \wedge N) \wedge \neg(B \wedge W) \wedge \neg(N \wedge W)) \in B(F)
\end{aligned}
$$

There are only six product configurations that satisfy $\chi$,

$$
\begin{aligned}
& \rho_{1}=\left(\bigwedge_{f \in O} f\right) \wedge B \wedge \neg N \wedge \neg W \wedge \neg S, \rho_{2}=\left(\bigwedge_{f \in O} f\right) \wedge B \wedge \neg N \wedge \neg W \wedge S, \\
& \rho_{3}=\left(\bigwedge_{f \in O} f\right) \wedge \neg B \wedge N \wedge \neg W \wedge \neg S, \rho_{4}=\left(\bigwedge_{f \in O} f\right) \wedge \neg B \wedge N \wedge \neg W \wedge S, \\
& \rho_{5}=\left(\bigwedge_{f \in O} f\right) \wedge \neg B \wedge \neg N \wedge W \wedge \neg S, \rho_{6}=\left(\bigwedge_{f \in O} f\right) \wedge \neg B \wedge \neg N \wedge W \wedge S .
\end{aligned}
$$

Figure 11 shows a table with abstracted product configurations and the set relation where each one is present.

The feature diagram graphically represents the feature relation while a feature model can be used to represent relevant information for testing. A feature model is defined as follows.

Definition 2.3.1. A feature model $F M$ is a triple $(F, \chi, \Lambda)$, where $F$ is the set of features, $\chi$ is the feature constraint of all feature relations, and $\Lambda$ is the set of product configurations that satisfies $\chi$.

Given two feature constraints $\omega_{a}$ and $\omega_{b}$, and $\Lambda_{a}, \Lambda_{b} \subseteq \Lambda$ are the subsets of configurations satisfying $\omega_{a}$ and $\omega_{b}$, respectively, we say that $\omega_{a}$ and $\omega_{b}$ are equivalent under $F M$ if $\Lambda_{a} \subseteq \Lambda_{b}$ and $\Lambda_{b} \subseteq \Lambda_{a}$. 


\subsubsection{Software Product Line Testing}

The SPL testing aims to examine core artifacts that are shared with many products, reusable modules, and their interactions. Thus, SPL testing encompasses VV\&T activities for the SPL architecture and its products (MCGREGOR, 2001).

The main advantages of the SPL approach are effective, low-cost mass production and customization of products. The SPL development process has a systematic software artifact reuse that increases productivity. However, new challenges arise in the testing activity. Testing is more challenging in SPLE than for individual systems (TEVANLINNA; TAINA; KAUPPINEN, 2004). In general, testing large SPLs demand substantial effort, and effective reuse is a challenge (LINDEN; SCHMIF; ROMMES, 2007). Some SPL testing challenges are:

- Variability management: suitable feature models, and variability representation in the product line architecture. Design and test models contain extra elements which represent all reusable elements. In general, a non-executable single model is created and then pruned for single products. Testing the whole model may be costly without proper testing techniques;

- Number of products: a large number of products that can be generated. Instead of testing all derivable products, only a representative subset of products are selected to testing the whole SPL (see Section 2.3.3);

- Dimensions of evolution: redundant tests in all three dimensions of evolution: level, version, and variant. In general, the SPL testing performs redundant tests in another test level (e.g., integration and system testing), previous versions of the same product, and previously designed products (RUNESON; ENGSTROM, 2012).

\section{Test Process Model}

The SPL testing checks core and reusable artifacts in two levels: domain testing; and application testing. The domain testing aims at detecting faults in artifacts produced in the domain engineering level and creates testing artifacts to be reused in application testing. The domain testing is similar to the testing of a single system. However, most core artifacts contain features that are mapped to some model elements and stubs are required to simulate unimplemented modules. The application testing reuses testing artifacts from domain testing to design and execute product-specific test-cases in the SUT. Domain and application testing levels are interdependent as one fault detected in the application domain might be a fault introduced in the domain testing.

Figure 12 presents the W-model conceptual test model for SPLs (JINHUA; QIONG; JING, 2008). The W-model is incorporated in both SPL development levels to supplement 


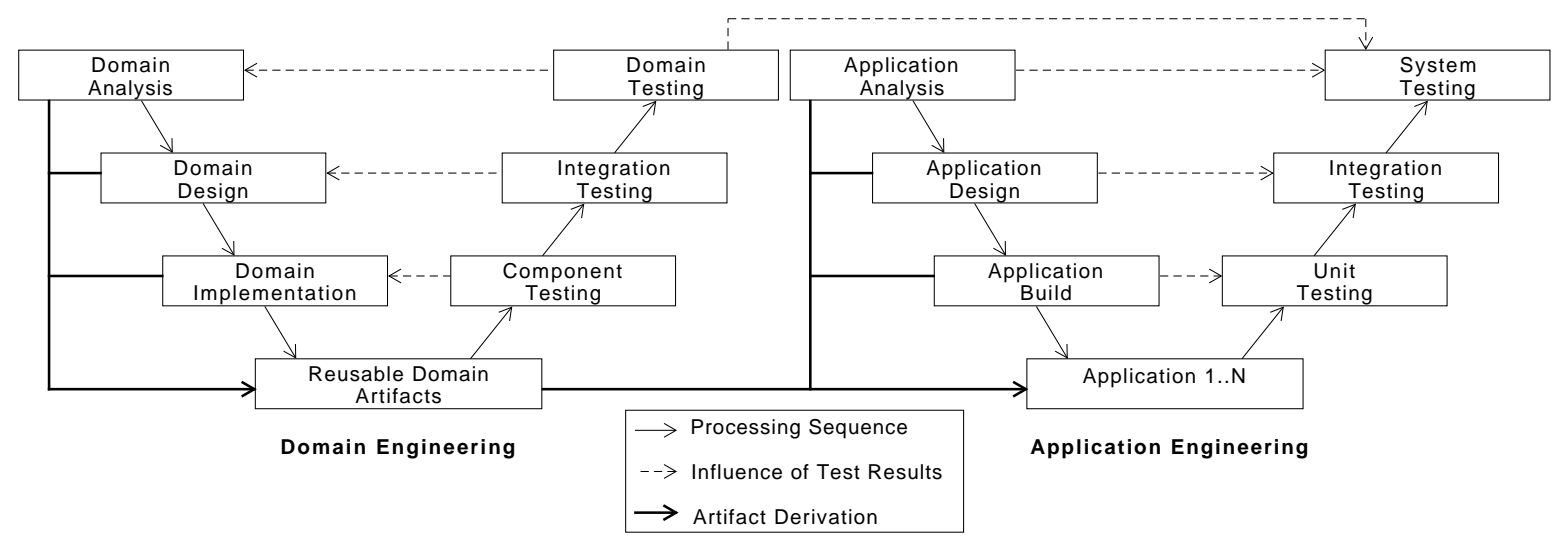

Figure 12 - The W-model for SPL testing (adapted from Jinhua, Qiong and Jing (2008)).

testing that results in a $\mathrm{W}$-form. Assets produced by domain and application engineering are tested on the levels of the $\mathrm{V}$-model. The left hand side of the $\mathrm{V}$-model includes the common abstraction levels of the software where tests are designed during the design and implementation of the system. The right hand side of the model binds testing levels to the abstraction levels in the implementation where designed tests are executed.

\section{Dimensions of Evolution}

Faults may be introduced in reusable or core artifacts. A fault introduced in a core artifact that was detected in a product means that fixes must be made considering all products, otherwise, all subsequent products may be faulty as well. Faults introduced in reusable artifacts must be traced back to the group of affected products. Moreover, the test plan must manage scope, resources, and time in three SPL dimensions of evolution (ENGSTROM; RUNESON, 2013):

- Level: test-cases are concretized and executed in several abstraction levels. Functionalities that are tested using abstract test models may be re-concretized and re-executed for another test model. The lack of traceability between test models in different test levels may result in test-case redundancy. Reuse-based strategies can be developed for test levels and consequently reduce test effort (PéREZ; KAISER, 2010);

- Time: test-cases are concretized and executed in different versions of a product over time. Regression testing approaches can be used to reuse test-cases for new versions and reduce test redundancy;

- Variants: test-cases are concretized and executed in different similar products. The SPL architecture has core artifacts that are present in every product turning them 


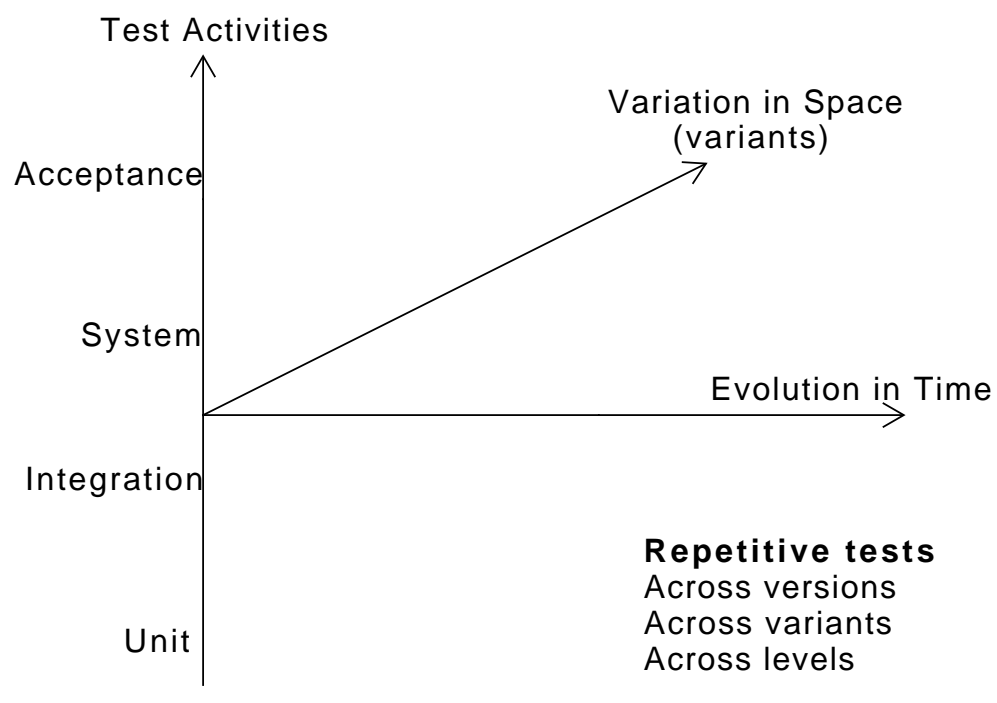

Figure 13 - 3D SPL test process model with dimensions of evolution (adapted from Engstrom and Runeson (2013)).

similar to each other. Thus, regression approaches can be adapted to reuse some test-cases between products.

Figure 13 presents the 3D SPL test process model with dimensions of evolution. SPL testing might have repetitive activities across version, variants, and levels (unit, integration, and system tests) as well redundant test artifacts (including test-cases) for requirements, design, interfaces, and implementation.

Test-case reuse strategies in SPL are essential to reduce testing costs. Ideally, a new derived product can be tested with little effort using automatic test-case reuse strategies (RAMLER; PUTSCHOGL, 2013).

\section{Test Strategies}

Testing exhaustively a large number of products that an SPL can derive is not viable because of project costs. In SPLs, a proper test-case reuse strategy is crucial due to the increasing test costs compared to the decreasing development costs. Thus, some products may be selected to test the SPL, or new derived products may be tested on demand. One naive strategy is to collect all test-cases of previous products and remove all obsolete and redundant tests. However, such approach is not economically viable to be applied assuming some test coverage criteria in large SPLs. There are three categories of SPL testing strategies (RUNESON; ENGSTROM, 2012): the anti-SPL philosophy; reuse-based; and combinatorial.

The anti-SPL testing strategies do not use artifact reuse and are useful for small 
SPLs, which are:

- Brute force: test the whole SPL architecture and all possible SPL products;

- Pure application (product-by-product - PBP): test every product independently without the reuse of test artifacts.

The reuse-based strategies reuse, increment, or adapt test artifacts created in the domain engineering level or from previously derived products, which are:

- Sample application: test the first product and other products are tested by reusing test artifacts from the first. In general, the most representative product (core product) is selected to be the first (best-selling), and other products are tested by analyzing differences using regression-based approaches. Some strategies are the incremental testing (UZUNCAOVA; KHURSHID; BATORY, 2010) and the delta modeling (LITY et al., 2012);

- Commonality and reuse: test all modules of common features and individually test modules of variable features. Modules that are not present in all products are tested once a product is about to be derived and deployed. MBT testing approaches use this strategy as test models can be created in the domain testing and reused in the application testing level. However, this strategy does not reuse test-cases between products. Thus, it can be combined with the application example strategy;

- Division of responsibility: test techniques are divided into domain and applications testing levels. For example, unit testing can be executed in the domain testing level while integration and system tests may be executed in the application testing level.

Combinatorial strategies identify and test representative subsets of products using heuristics, which are:

- Pairwise testing: test every combination of two features that interact with each other (OSTER; MARKERT; RITTER, 2010).

- T-wise testing: test every combination of several T (more than 2) features that interacts with each other (PERROUIN et al., 2010).

Some SPL test strategies can be combined to reduce testing costs. Large SPLs may combine more than one strategy to create a test plan. Figure 14 illustrates four classic Pohl's (POHL; BÖCKLE; LINDEN, 2005) SPL test strategies. In brute force strategy, all related SPL test artifacts are tested, while pure application each vertical arrow represent 


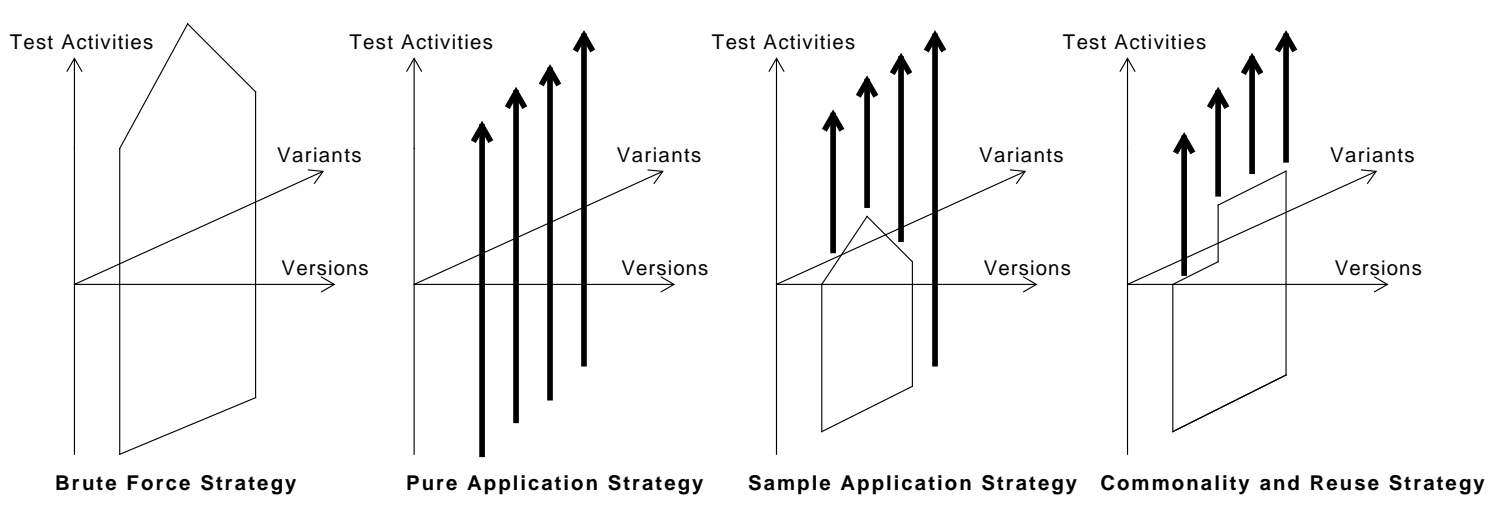

Figure 14 - Classic SPL testing strategies applied to the 3D model (adapted from Runeson and Engstrom (2012)).

the testing flow of a product. In sample application strategy one product is tested, and other products reuse test artifacts from the first, while commonality and reuse strategy tests all common artifacts and then test reusable artifacts on demand in each product.

\section{Regression-based SPL Testing}

The literature proposes some strategies for improving test efficiency but lacks real life evaluations (ENGSTRÖM; RUNESON, 2011). Research on regression testing, which addresses similar problems, offers some empirically evaluated approaches. With existing regression testing approaches it is possible to provide automated decision support in a few specific cases. Figure 15 shows the generic interaction between the SPL architecture and products for testing. Test-cases and the test plan are created for the SPL architecture and then specialized for each product.

Some SPL testing approaches (LOCHAU; KAMISCHKE, 2012; LOCHAU et al., 2014) make use of techniques inspired from regression testing to test new products. Such approaches explore version and variant dimensions of evolution. Few other approaches such as Silveira Neto et al. (Silveira Neto et al., 2010) developed regression testing approaches based on architecture specification. They identify that the effort to apply the corrective scenario is greater than the progressive scenario. Moreover, some thesis (KIM et al., 2006; HEIDER et al., 2012; REMMEL et al., 2012; LOCHAU et al., 2012) publish tools, environment, and frameworks for SPL-based regression testing during variability modeling to instantiate products.

\subsection{Concluding Remarks}

In this chapter, we presented all basic definitions and concepts regarding software testing, model-based testing, and software product lines. In detail, we present preliminaries 


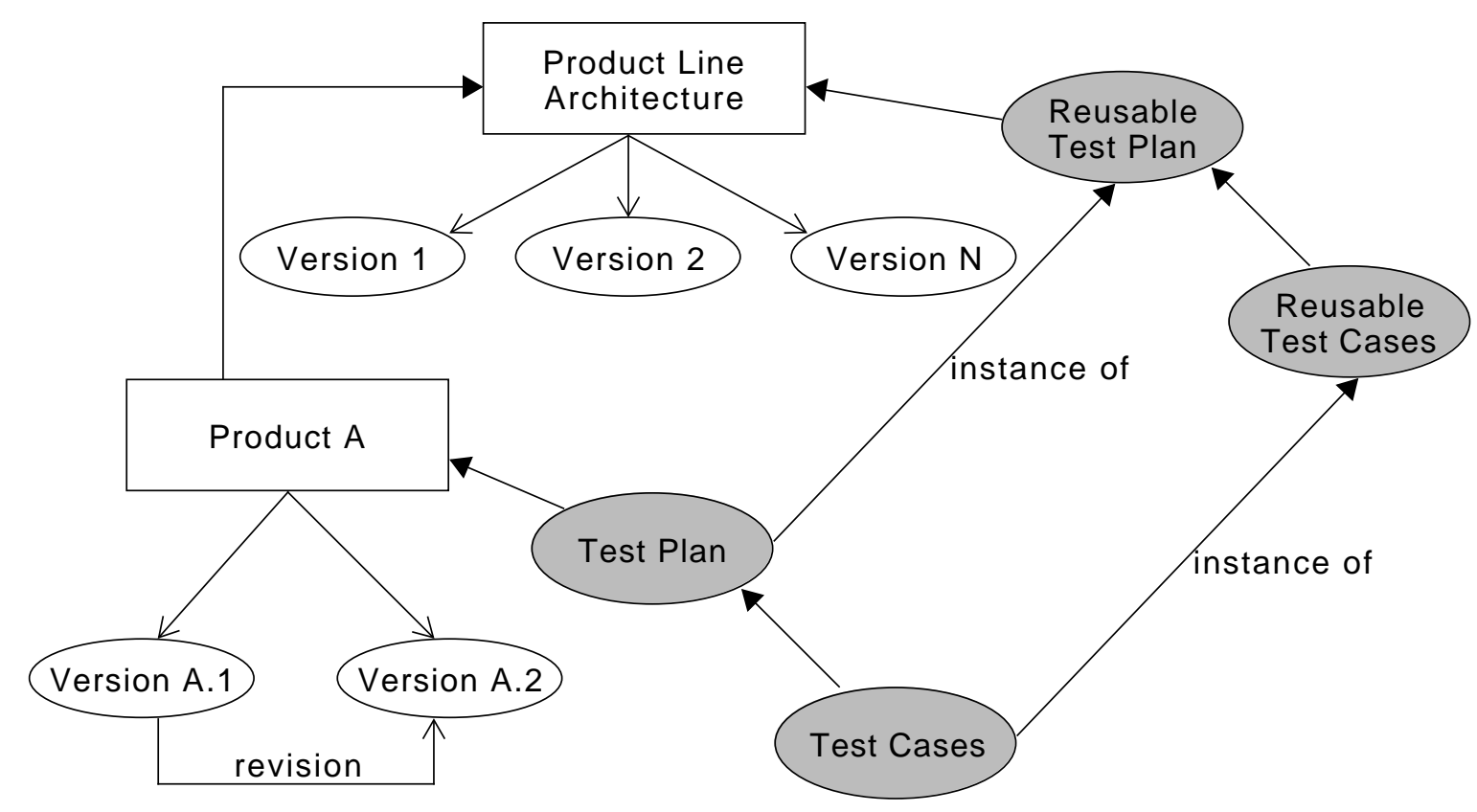

Figure 15 - SPL testing artifacts interaction (adapted from McGregor (2001)).

for test design based on state machines. Then, we present basic concepts, challenges, and strategies for SPL testing. 
Part II

Theory 

CHAPTER

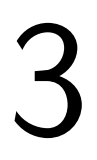

\section{REGRESSION-BASED TESTING FOR SOFTWARE PRODUCT LINES}

In this chapter it is presented a test reuse strategy named Incremental Regressionbased Testing for Software Product Lines (IRT-SPL) that aims at reducing the test effort of newly designed SPL products by reducing the number of new test-cases that need to be concretized for conformance testing. To this end, the reuse of test-cases is maximized by processing concretized test-cases of all old products and incrementing some of them to obtain a small set of test-cases to concretize.

The remainder of this chapter is organized as follows. Section 3.1 presents how test effort is calculated while Section 3.2 presents our test reuse strategy and the selection algorithm. Then, Section 3.3 provides a regression-based test design example using our strategy.

\subsection{Test Effort Calculation}

Given a new product configuration to test, first, we check the changed behavior to ensure that they behave as intended by concretizing and executing a set of new test-cases. Then, to ensure that the unchanged behavior was not affected by modifications we execute a set of test-cases to retest such behavior.

Definition 3.1.1. Test-case and test-suite size. The size of a test-case $\alpha \in I^{*}$ denoted by $|\alpha|$ is calculated by the number of inputs that it contains, i.e., $|\alpha|=k, \alpha=x_{1}, \ldots, x_{k}$. Similarly, $|T|$ is the size of a test-suite $T$ calculated by the sum of all test-cases plus the reset operation for each maximal test-case, i.e., $|T|=\sum(|\alpha|+1), \alpha \in T, \nexists_{\beta \in T} \bullet \beta=\alpha \gamma \wedge \gamma \neq \varepsilon$.

Example 7. Given a test-suite $T=\{a, b, c ; a, c\}$ the size of $T$ is $|T|=(|a, b, c|+1)+$ $(|a, c|+1)=7$. 
Definition 3.1.2. The effort to test a new product configuration is the sum of testcases that have to be executed plus those that have to be concretized (turn abstract to executable) times a value $x$ (manual concretization value over execution), i.e., effort= execution $+($ concrete $* x)$.

Case studies (UTTING; LEGEARD, 2006; Graham D., 2012) show that the value $x$ is around 200. Execution cost is calculated based on the number of test-cases that have to be executed for both changed and unchanged behavior. Concretization cost is calculated from new test-cases.

Definition 3.1.3. Execution and concretization costs. Given a prefix-closed test-suite $T$, a set of new test-cases $N T \subseteq T$ and a set of concretized test-cases $D=T \backslash N T$, the execution cost is equivalent to the size of $|T|$, i.e., execution $=|T|$. The concretization cost is calculated from new test-cases $\alpha \in N T$ that have to be concretized. If a proper prefix $\beta$ of a new test-case $\alpha=\beta \gamma, \gamma \neq \varepsilon$ was already concretized before, i.e., $\beta \in D$, then we reuse $\beta$ and the concretization cost is the sum of the size of all suffixes $\gamma$, i.e., concrete $=\sum|\gamma|$.

Example 8. Given a test-suite $T=\operatorname{pref}\{a, b, c ; a, c ; a, d\}$ and a subset of new test-cases $N T=\{a, d\}$, the concretization cost is concrete $=|d|=1$.

\subsection{Regression-based Testing for Software Product Lines}

The Incremental Regression-based Testing for Software Product Lines (IRT-SPL) strategy was inspired by earlier approaches in this domain (SIMAO; PETRENKO, 2010; UZUNCAOVA; KHURSHID; BATORY, 2010; CAPELLARI et al., 2012; LOCHAU et al., 2014) and developed to improve the reuse of tests cases to reduce concretization effort. We use incremental test generation methods (to increment concretized tests) for full fault coverage criteria. Figure 16 (a) presents the main steps of IRT-SPL and (b) presents an overview of the selection algorithm.

Given a new product configuration $p \in \Lambda$ that requires testing, for checking its conformance we design the test model as an FSM $M$, obtain all defined test-cases $D \subseteq \Omega_{M}$ that were concretized in old products, and execute the following sequence of steps and conditions:

Step 1: Process all defined test-cases of $D$ to find divergent, convergent, and convergence-preserving test-cases (SIMAO; PETRENKO, 2010). Also, initialize the set of new test-cases $N T=\varnothing$.

Condition 1: Is set $D n$-complete for $M$ ? When the answer is false, move to Step 2; otherwise, copy $D$ to set $T$ and move to Step 3. 


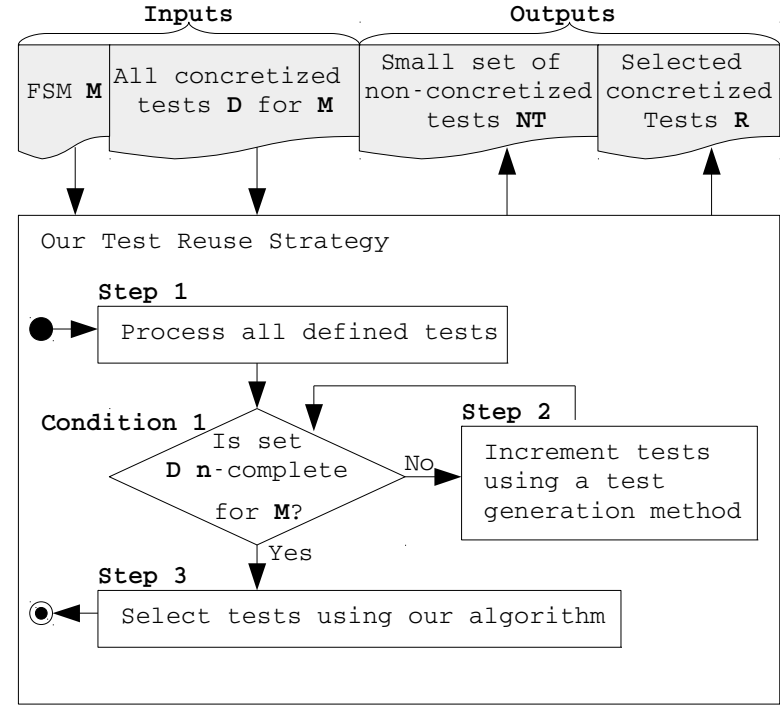

(a)

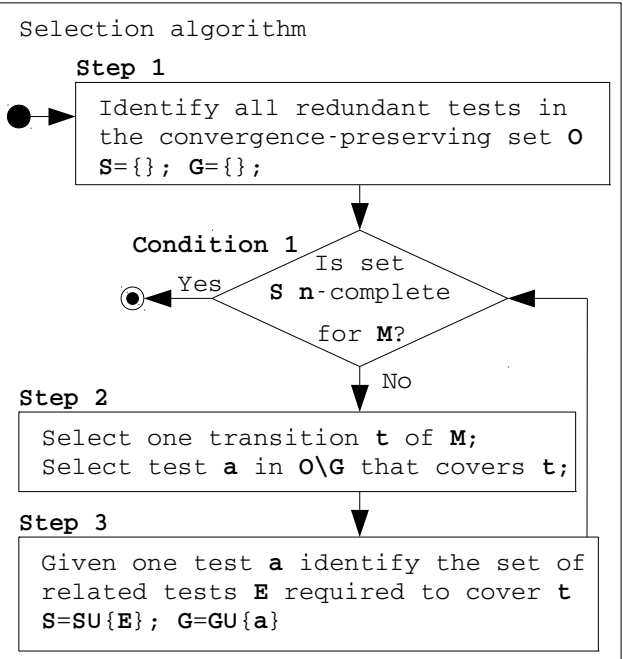

(b)

Figure 16 - Proposed regression-based test reuse strategy.

Step 2: Increment test-cases using a test generation method. Incremental test generation methods use a cost calculation that decides which new test-case gives a small increment based on test-cases of $D$. Thus, we used the P method (SIMAO; PETRENKO, 2010) for this step. New test-cases are incremented from $D$ and put in $N T$, resulting in an $n$-complete test-suite $T=D \cup N T$.

Step 3: Obtain test-cases using our selection algorithm. Execute the selection algorithm using $M$ and $T$ as parameters, obtain the $n$-complete test-suite $S \subseteq T$ and return $R=S-N T$ as the set of selected concretized test-cases and $N T$ as the set of non-concretized test-cases.

In general, incremental test-case generation algorithms check available test-cases that can be incremented. Sometimes two equivalent test-cases (with the same size for a given test criteria) can be used to increment and generate a new test-case. Selecting one of such tests might result in a larger/smaller test-suite at the end of the process. Thus, we pick the first test-case (greedy) as generating a minimal test-suite (even by incrementing test-cases) can lead to an exponential number of situations.

\subsubsection{Selection Algorithm}

The selection algorithm proposed was developed on the last step of the IRTSPL strategy. Given an FSM $M$, an $n$-complete test-suite $T$ for $M$, and an initialized convergence-preserving transition cover set $O \subseteq T$, we select non-redundant test-cases of $O$ to obtain the $n$-complete test-suite $S \subseteq T$. Set $O$ is the one that controls model coverage regarding the full fault coverage which might contain redundant tests. Each test in $O$ requires a set of tests cases in $T$ to maintain the set property considering $M$. Thus, 
we select a subset $G \subseteq O$ resulting set $S \subseteq T$.

The main steps are:

Step 1: Identify all redundant test-cases. All test-cases of $O$ that cover each transition of $M$ are identified. Also, the resulting test-suite $S$ and set $G \subseteq O$ are initialized.

Condition 1: Is set $S$ n-complete for $M$ ? The set $G \subseteq O$ must be a transition cover set with a convergence-preserving property (SIMAO; PETRENKO, 2010). When the answer is true, return $S$; otherwise, move to Step 2.

Step 2: Select one transition $t$ of $M$. Only transitions not covered by $G$ are selected. Select a test-case $a \in O \backslash G$ that covers $t$. Sometimes there are several redundant test-cases in $O$ that cover $t$. Then, select the test-case that gives the smallest increment of test-cases for $S$. For equivalent test-cases (which lead to the same size) we just select the first in the line (greedy).

Step 3: Given a test-case $a$ from Step 2, identify the set of related test-cases $E \subseteq T$ required to cover $t$. Then, $a$ is included in $G$ and $E$ in $S$.

The relations (sets of sets) $C$ and $D$ represent subsets of $\Im_{n}(T)$-convergent and $\Im_{n}(T)$-divergent sets, respectively. In the selection algorithm section we use $D$ to represent something else, and for $\chi$ as well (from the original rules), which is a prefix, not a feature constraint. The relation $D$ is initially the set of all pairs of $T$-separated tests according to Lemma 1. Next, a $M$-divergent state cover set $K$ with $n$ tests is identified. The relation $C$ is the set of all pairs of $\Im_{n}(T)$-convergent tests according to Lemma 2 (including the identity set where $(\alpha, \alpha) \in C)$. We used 10 rules that were introduced in (SIMAO; PETRENKO, 2010) to identify extra pairs of convergent and divergent test pairs in $C$ and $D$, respectively. The ten rules are (SIMAO; PETRENKO, 2010):

1. If $(\alpha, \beta)$ is added to $C$, for each $(\alpha, \chi) \in C$, add $(\beta, \chi)$ to $C$.

2. If $(\alpha, \beta)$ is added to $C$, then, for all their common extensions $\alpha \varphi, \beta \varphi \in T$, add $(\alpha \varphi, \beta \varphi)$ to $C$.

3. If $(\alpha, \beta)$ is added to $D$, and they are common extensions of tests $\alpha^{\prime}$ and $\beta^{\prime}$, then add $\left(\alpha^{\prime}, \beta^{\prime}\right)$ to $D$.

4. If $(\alpha, \beta)$ is added to $C$, then, for each $\chi \in T$ if $(\alpha, \chi) \in D$, add $(\beta, \chi)$ to $D$; if $(\beta, \chi) \in D$, add $(\alpha, \chi)$ to $D$.

5. If $(\alpha, \beta)$ is added to $D$, then, for each $\chi \in T$ if $(\alpha, \chi) \in C$, add $(\beta, \chi)$ to $D$; if $(\beta, \chi) \in C$, add $(\alpha, \chi)$ to $D$.

6. If $(\alpha, \beta)$, with $\alpha \leq \beta$, is added to $D$ and there exists sequence $\varphi$ and $k>1$, such that $\beta=\alpha \varphi^{k}$, then add $(\alpha, \alpha \varphi)$ to $D$. 
7. If $(\alpha, \alpha \beta \gamma)$ is added to $C$, and $(\alpha, \alpha \gamma) \in D$, then add $(\alpha, \alpha \beta)$ to $D$.

8. If $(\alpha, \alpha \gamma)$ is added to $D$, then, for each sequence $\beta$ such that $(\alpha, \alpha \beta \gamma) \in C$, add $(\alpha, \alpha \beta)$ to $D$.

9. If $(\alpha, \alpha \gamma)$ is added to $C$, then, for each sequence $\beta$ such that $(\beta, \beta \gamma) \in D$, add $(\alpha, \beta)$ to $D$.

10. If $(\beta, \beta \gamma)$ is added to $D$, then, for each sequence $\alpha$ such that $(\alpha, \alpha \gamma) \in C$, add $(\alpha, \beta)$ to $D$.

The relation $C_{\cup}(K)=\{\beta \mid(\alpha, \beta) \in C, \alpha \in K\}$ is an $\Im_{n}(T)$-convergence-preserving set for $M$ according to Definition 2.2.12. Moreover, we define sets $V \subseteq C \cup D$ for verified pairs of tests of $C$ and $D, G \subseteq C_{\cup}(K)$ for goal coverage, and $S \subseteq T$ to store the selected tests of $T$. To identify convergent and divergent pairs that were added to $C$ and $D$ by rules 1-10, we use designed inverse rules to trace tests back to $T$-separated pairs where they originally came from.

11. If $(\beta, \chi) \in C$ was added by rule 1 , then check $(\alpha, \beta),(\alpha, \chi) \in C$

12. If $(\alpha \varphi, \beta \varphi) \in C$ was added by rule 2 , then check $(\alpha, \beta) \in C$

13. If $(\alpha, \beta) \in D$ was added by rule 3 , then check $(\alpha \gamma, \beta \gamma) \in D$.

14. If $(\alpha, \chi) \in D$ was added by rule 4 , then $\operatorname{check}(\beta, \chi) \in D,(\alpha, \beta) \in C$.

15. If $(\alpha, \chi) \in D$ was added by rule 5 , then check $(\beta, \chi) \in C,(\alpha, \beta) \in D$.

16. If $(\alpha, \alpha \varphi) \in D$ was added by rule 6 , then check $(\alpha, \beta) \in D$, with $\alpha \leq \beta, \beta=\alpha \varphi^{k}, k>$ 1.

17. If $(\alpha, \alpha \beta) \in D$ was added by rule 7 , then check $(\alpha, \alpha \gamma) \in D,(\alpha, \alpha \beta \gamma) \in C$.

18. If $(\alpha, \alpha \beta) \in D$ was added by rule 8 , then check $(\alpha, \alpha \gamma) \in D,(\alpha, \alpha \beta \gamma) \in C$.

19. If $(\alpha, \beta) \in D$ was added by rule 9 , then check $(\beta, \beta \gamma) \in D,(\alpha, \alpha \gamma) \in C$.

20. If $(\alpha, \beta) \in D$ was added by rule 10 , then check $(\beta, \beta \gamma) \in D,(\alpha, \alpha \gamma) \in C$.

The main detailed steps of the selection algorithm are presented in Figure 17.

Given an FSM $M$ and $n$-complete test-suite $T$ for $M$, initially the algorithm processes $T$ and identify sets $D, K, C$, and $C_{\cup}(K)$. Some tests may be added in those sets (except $K$ ) by rules $1-10$. The verification set $V$ start empty, coverage set $G$ initialized, and the resulting selected set $S$ empty. 


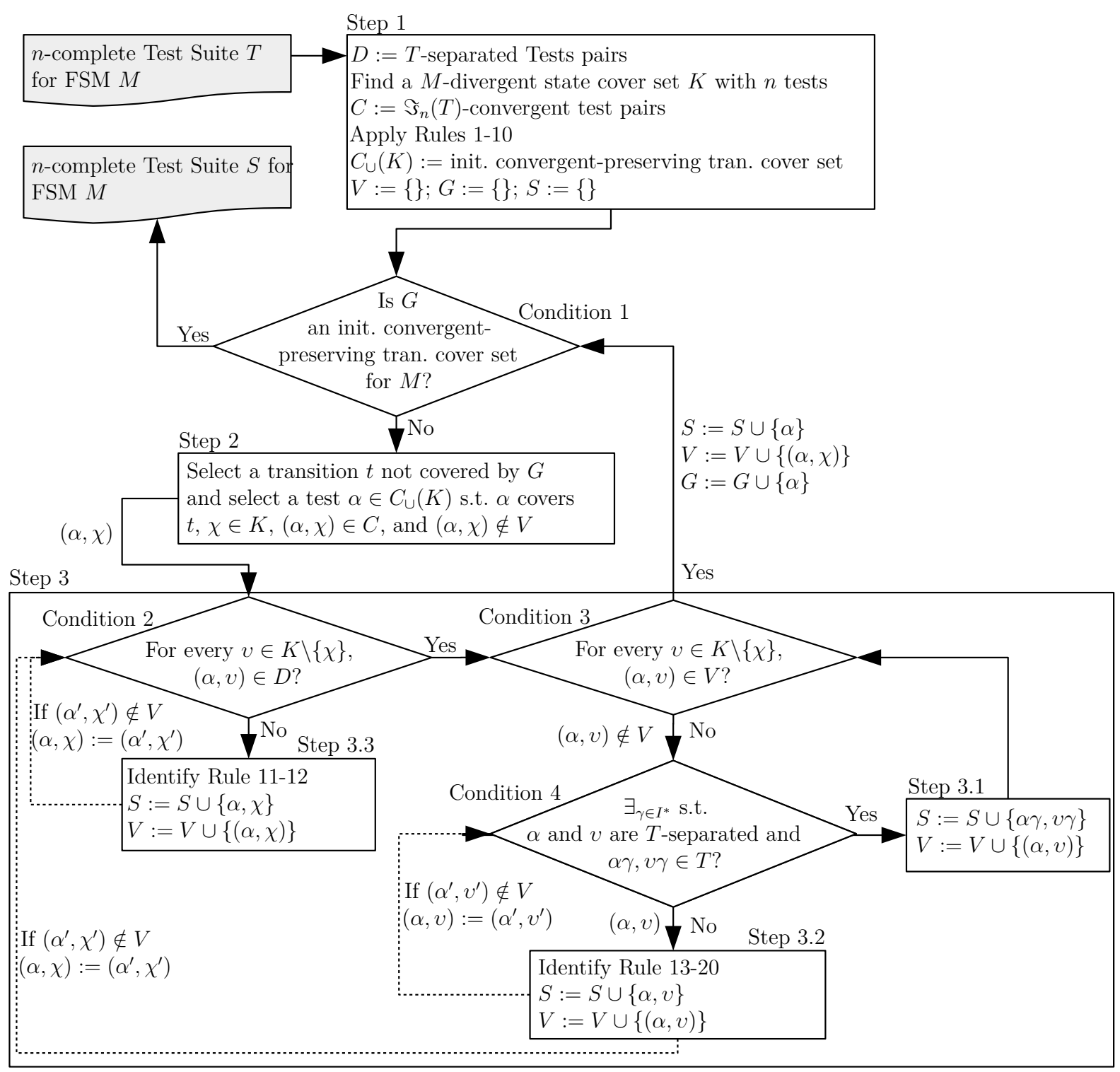

Figure 17 - Algorithm for reducing an $n$-complete test-suite.

Condition 1 checks whether $G$ meets the condition of a $n$-complete test-suite, and since it is initially empty Step 2 is executed. To populate $G$ first select a transition $t$ not covered by $G$ and select the smallest first test $\alpha \in C_{\cup}(K)$ such that $\alpha$ covers $t, \chi \in K$, $(\alpha, \chi) \in C$, and $(\alpha, \chi) \notin V$. Then, we check whether the pair $(\alpha, \chi)$ was added to $C$ by some rule or by Lemma 2. Let Condition 2 be true for $(\alpha, \chi)$, then on Condition 3 no pair $(\alpha, v)$ is true as $V$ is empty at this point. Moving to Condition 4, let it be true, then tests $\alpha \gamma, v \gamma$ are added to $S$ and $(\alpha, v)$ added to $V$ marking this pair as visited and checked. Back to Condition 3, assuming that every other pair $(\alpha, v)$ make Condition 4 true, then after verifying them Condition 3 turns true, $(\alpha, \chi)$ is added to $V, \alpha$ is added to $S$ and $G$ finishing the basic cycle for $t$.

Let Step 2 select a test pair not verified $(\beta, \chi) \in C$ that was added to $C$ by rule 1. If Condition 2 turn false, then Step 5 identify pairs e.g., $(\alpha, \beta),(\alpha, \chi) \in C$ by rule 11 
that were used to add $(\beta, \chi)$ in $C$, then, add $\beta$ and $\chi$ to $S$ and put $(\beta, \chi)$ in $S$ to mark as verified. First, if $(\alpha, \beta) \notin V$ then the execution continues on Condition 2 for $(\alpha, \beta)$ instead of $(\beta, \chi)$ and $(\alpha, \chi)$ enters in a waiting state. If $(\alpha, \beta) \in V$, then $(\alpha, \beta)$ is ignored, and if $(\alpha, \chi) \notin V$ then the execution continues on Condition 2 for $(\alpha, \chi)$ instead of $(\beta, \chi)$. However, if $(\alpha, \beta),(\alpha, \chi) \in V$ then the execution returns to the last waiting state. If there is no waiting point to return, then the execution stops with a failure, and $T$ is not $n$-complete.

On Condition 3 for every pair $(\alpha, v) \notin V$ select one and use as input for Condition 4 and put the rest in a waiting state. If Condition 4 is false, then identify which pairs of tests were responsible on the addition of $(\alpha, v)$ to $D$, then add $\alpha$ and $v$ to $S$, and $(\alpha, v)$ to $V$ to mark as verified. For example, if $(\alpha, v)$ was triggered by Rule 14 , then $(\beta, v) \in D,(\alpha, \beta) \in C$. If $(\beta, v) \notin V$ then the execution continues on Condition 4 for $(\beta, v)$ and $(\alpha, \beta)$ enters in a waiting state. If $(\beta, v) \in V$, then $(\beta, v)$ is ignored, and if $(\alpha, \beta) \notin V$ then the execution continues on Condition 2 for $(\alpha, \beta)$. However, if $(\beta, v),(\alpha, \beta) \in V$ then the execution returns to the last waiting state. If there is no waiting point to return, then the execution stops with a failure, and $T$ is not $n$-complete.

\subsubsection{Test-suite Analysis}

In this subsection, we state and prove that the selection algorithm terminates with a $n$-complete test-suite.

Theorem 2. Given an $n$-complete test-suite $T$ for an FSM $M$, the Algorithm 17 ends with an $n$-complete test-suite $S \subseteq T$.

Proof. The algorithm has four loops. Each loop is executed in a finite number of times, and then we prove that the selected test-suite is $n$-complete as the original test-suite used as input.

Loop 1 represent the execution where Condition 1 is false and Conditions 2 and 3 are true, i.e. select tests to populate $G$. As the selected pair $(\alpha, \chi)$ cannot be in the set of verified pairs $V$ and the number of pairs in $C$ is finite, at the end of the loop $(\alpha, \chi)$ is put in $V$ and cannot be selected again. Thus, the number of executions of Loop 1 is bounded.

Loop 2 represent the execution where Condition 3 is false and Conditions 4 is true, i.e. the pair $(\alpha, v)$ is $T$-separated. On Step $3(\alpha, v)$ is put in $V$ to mark as verified. As the number of pairs in $D$ is finite, the Loop is bounded to $D$ size.

Loop 3 represent the execution where Condition 2 is false, i.e. the pair $(\alpha, \chi)$ was added to $C$ by rules. On Step $5(\alpha, \chi)$ is put in $V$ to mark as verified. The loop continues only if one of the required pairs for $(\alpha, \chi)$ is not in $V$, otherwise, the execution returns to 


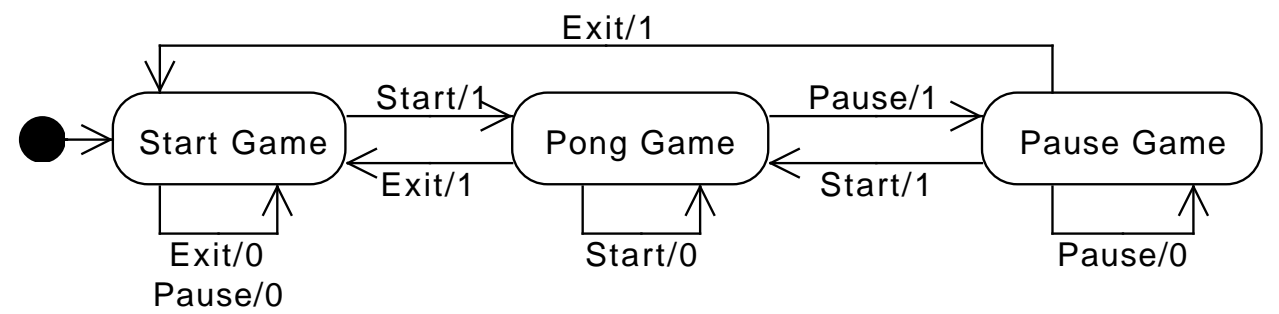

Figure $18-$ FSM of the third product configuration of AGM.

the last waiting state. As the number of pairs in $C$ is finite, the Loop is bounded to the size of $C$.

Loop 4 represent the execution where Condition 4 is false, i.e. the pair $(\alpha, v)$ was added to $D$ by rules. On Step $4(\alpha, v)$ is put in $V$ to mark as verified. The loop continues only if one of the responsible pairs for $(\alpha, v)$ is not in $V$, otherwise, the execution returns to the last waiting state. As the number of pairs in $D$ is finite, the Loop is bounded to $D$ size.

Given an FSM $M$, the selected test-suite $S$ is $n$-complete. When the algorithm ends, Condition 1 holds and $G$ is an initialized $\Im_{n}(S)$-convergent-preserving transition cover set for $M$, by Definition 1 . For every test $\alpha$ added to $G$, a subset of tests that made $\alpha \in C_{\cup}(K)$ were added to $S$ resulting in a $n$-complete test-suite for $M$.

The time required to execute the algorithm is similar to the $\mathrm{P}$ method since several steps have the same complexity executed in inverse order. However, there are two exceptions: the state cover set $K$ that was identified by simple breadth-first search instead of a clique, and we only apply the first 10 rules once in the first step instead of each step of $\mathrm{P}$ method. Check in (SIMAO; PETRENKO, 2010) for more details about the complexity of each step.

\subsection{Test Design Example}

There are six possible products that can be derived from the AGM SPL presented in Section 2.3.2. Assume that two products were already tested for Brickles, with and without the Save option. Figure 18 presents the FSM $M_{3}$ of the third product configuration $\rho_{3}$ (Example 6) where the Pong[N] rule was selected and the Save[S] option discarded. This test model is an abstracted version of the design model where observable events are represented by inputs and the correspondent outputs. The inputs are in-game commands, while the outputs 0 and 1 are abstract captured responses. It is straightforward to check that $M_{3}$ is a deterministic, complete, initially connected and minimal FSM.

Given the FSM $M_{3}$, the IRT-SPL approach allows the test design of few extra new 


\begin{tabular}{|c|c|c|}
\hline 1 & PS,EX & 1 PS, EX \\
\hline 2 & PS, PS, EX & 2 PS, PS, EX \\
\hline 3 & EX, PS, EX & 3 EX, PS, EX \\
\hline 4 & SV,PS, EX & 4 SV, PS, EX \\
\hline 5 & SG, PS , PS , EX & SG , PS , PS, EX \\
\hline 6 & $\mathrm{SG}, \mathrm{EX}, \mathrm{PS}, \mathrm{EX}$ & 6 SG, EX, PS, EX \\
\hline 7 & SG, SV, PS, EX & SG , SV , PS, EX \\
\hline 8 & $\mathrm{SG}, \mathrm{SG}, \mathrm{PS}, \mathrm{EX}$ & $8 \quad \mathrm{SG}, \mathrm{SG}, \mathrm{PS}, \mathrm{EX}$ \\
\hline 9 & $\mathrm{SG}, \mathrm{PS}, \mathrm{EX}, \mathrm{PS}, \mathrm{EX}$ & $9 \quad$ SG, PS, EX, PS , EX \\
\hline 10 & SG , PS , SG , PS , EX & 10 SG, PS , SG , PS , EX \\
\hline 11 & SG , PS , SV , EX, PS, EX , SG , PS , EX & 11 SG , PS , SV , EX , PS , EX , SG , PS , EX \\
\hline 12 & $\mathrm{SG}, \mathrm{SV}, \mathrm{SV}, \mathrm{PS}, \mathrm{EX}, \mathrm{PS}, \mathrm{EX}, \mathrm{SV}, \mathrm{PS}, \mathrm{EX}$ & $\begin{array}{ll}12 & \text { SG, SV , SV , PS, EX, PS, EX, SV , PS, EX } \\
13 & \text { SG , PS, PS, PS }\end{array}$ \\
\hline & (a) & $(\mathrm{b})$ \\
\hline 1 & PS, EX & 1 PS, EX \\
\hline 2 & EX,PS, EX & $2 \mathrm{EX}, \mathrm{PS}, \mathrm{EX}$ \\
\hline 3 & $\mathrm{SG}, \mathrm{SG}, \mathrm{PS}$ & 3 SG, SG,PS \\
\hline 4 & SG , PS , PS , EX & 4 SG,PS, PS, EX \\
\hline 5 & SG , EX, PS, EX & 5 SG, EX, PS, EX \\
\hline 6 & $\mathrm{SG}, \mathrm{PS}, \mathrm{SG}, \mathrm{PS}$ & $6 \mathrm{SG}, \mathrm{PS}, \mathrm{SG}, \mathrm{PS}$ \\
\hline 7 & SG , PS, EX, PS , EX & 7 SG,PS, EX, PS, EX \\
\hline 8 & SG ,PS, PS, PS & \\
\hline
\end{tabular}

Figure 19 - Example of regression-based test sets.

test-cases. Figure 19 shows four test-case sets generated by IRT-SPL for $M_{3}$ : (a) defined test-cases $D$ for $M_{3}$ that were already concretized before; (b) $n$-complete test-suite $T$ for $M_{3}$ generated by $\mathrm{P}$ method by incrementing $D$; (c) a selected $n$-complete test-suite $S \subseteq T$ for $M_{3}$ generated by our selection algorithm; and (d) test-case set $R$ for retest unchanged behavior. Test-cases were simplified for readability and each input corresponds to: (i) SG - Start; (ii) PS - Pause; (iii) EX - Exit; and (iv) SV - Save. The resulting test-suite of ITR-SPL is item (c).

Note that the difference between (a) and (b) is the addition of Line 13 on (b) and only the last inputs are in bold. Since all four test-case sets are prefix-closed, every prefix is also a test-case to be counted. Notice that the prefix $S G, P S, P S$ of Line 13 (b) is already present on Line 5 as a prefix that can be reused. On (c), the algorithm to select concretized test-cases is executed using as input $M_{3}$ and (b), such that it keeps new test-cases and reduce the test-cases set of (a) as some of them are redundant to cover the unchanged behavior. Thus, the set of new test-cases is composed in Line 8 (c) (i.e., SG,PS,PS,PS) and the test-case set for the retest is $(d)$.

The effort required to check the conformance of $p_{3}$ using $M_{3}$ and IRT-SPL is calculated by effort $=($ concrete $* x)+$ execution. Assuming that $x=10$, we have the set of new test-cases $N T=\{S G, P S, P S, P S\}$, the reduced test-suite $S$ is Figure 19 (c), execution $=|S|=37$, concrete $=|\{P S\}|=1$, resulting in effort $=(1 * 10)+37=47$.

Further experimental results are provided in Section 7.1. 


\subsection{Concluding Remarks}

In this chapter was presented the test reuse strategy named Incremental Regressionbased Testing for Software Product Lines (IRT- SPL) that aims at reducing test effort on checking conformance of several SPL products. Test-cases of previously designed products can be efficiently reused for a newly designed product using incremental test-case generation methods to reduce the number of required test-cases for concretization. We assume that manual concretization of test-cases is several times more expensive than executing the same test-case. Thus, the effort required to test a new product is directly related to concretization costs.

Finite State Machines were used to represent the abstract behavior of the products as test models. To maximize reuse of test-cases, all test-cases that were concretized before are analyzed, and some of them are selected to retest the unchanged behavior of the new product under test. Thus, our strategy also contains a selection algorithm to perform the selection of non-redundant concretized test-cases. 


\section{FEATURED FINITE STATE MACHINES}

Variants of the finite state machine (FSM) model have been extensively used to describe the behavior of different domains. In particular, several model-based testing approaches (OSTER et al., 2012a) have been developed to support test design and execution for SPLs. Most of such techniques require several validation properties to hold for the underlying test models. However, the full fault coverage for FSMs does not adapt well to other formalisms. In this thesis, an extension of the FSM test model for software product lines (SPLs) is presented, named featured finite state machine (FFSM). As the first step towards using FFSMs as test models, we define feature-oriented variants of basic test model validation criteria. We show how the high-level validation properties coincide with the necessary properties of the product FSMs. Then, we introduce family-based test design based on state machines.

The remainder of this chapter is organized as follows. Section 4.1 introduces basic syntax and semantics of FFSMs, followed by definitions of model derivation for configurable test models in Section 4.2. Section 4.3 presents validations properties required for test-case generation, and finally test design for configurable test-cases for the full fault coverage in Section 4.4 .

\subsection{Syntax and Semantics}

A Featured Finite State Machine (FFSM) (FRAGAL; SIMAO; MOUSAVI, 2016) is an extension of a Finite State Machine (FSM) in which states and transitions are annotated/embedded with feature constraints. The syntax of an FFSM is defined as follows.

Definition 4.1.1. An FFSM is a 6-tuple $\left(F M, C, c_{0}, Y, O, \Gamma\right)$, where

1. $F M=(F, \chi, \Lambda)$ is a feature model (Definition 2.3.1), 
2. $C \subseteq S \times B(F)$ is a finite set of conditional states, where $S$ is a finite set of state labels, $B(F)$ is the set of all feature constraints, and $C$ satisfies the following condition:

$$
\forall(s, \varphi) \in C \bullet \exists_{\rho \in \Lambda} \bullet \rho \vDash \varphi
$$

3. $c_{0}=\left(s_{0}\right.$, true $) \in C$ is the initial conditional state,

4. $Y \subseteq I \times B(F)$ is a finite set of conditional inputs, where $I$ is the set of input labels,

5. $O$ is a finite set of outputs,

6. $\Gamma \subseteq C \times Y \times O \times C$ is the set of conditional transitions satisfying the following condition:

$$
\forall\left((s, \varphi),\left(x, \varphi^{\prime \prime}\right), o,\left(s^{\prime}, \varphi^{\prime}\right)\right) \in \Gamma \bullet \exists_{\rho \in \Lambda} \bullet \rho \vDash\left(\varphi \wedge \varphi^{\prime} \wedge \varphi^{\prime \prime}\right)
$$

The above-given two conditions ensure that every conditional state and every conditional transition is present in at least one valid product of the SPL. A conditional state $c=(s, \varphi) \in C$ is alternatively denoted by $s[\varphi]$. A conditional transition from conditional state $c$ to $c^{\prime}$ with conditional input $y$ and output $o t=\left(c, y, o, c^{\prime}\right)$ is alternatively denoted by $x[\varphi] / o$ or $c \underset{o}{\stackrel{y}{\rightarrow}} c^{\prime}$. The operators of feature constraints are denoted by $\& \&$ (and), $\|$ (or), and ! (not). Omitted feature conditions mean that the condition is true, i.e., state $s$ is equivalent to $(s$, true $) \in C$, and transition $\underset{o}{\rightarrow}$ is equivalent to $\underset{o}{\stackrel{(x, t r u e)}{\rightarrow}}$.

Example 9. Figure 20 shows an FFSM for the AGM SPL. Figure 10 shows one optional feature $S$ for Save Game, and three alternative xor features: $N$ for Pong; $W$ for Bowling; and $B$ for Brickles. Conditional state (Save Game, $S$ ) represent the behavior of products which have the $S$ feature. Any conditional transition reaching or leaving (Save Game, $S$ ) also has the $S$ constraint. A product-specific transitions (Save Game, $S) \underset{1}{\stackrel{(\text { Pause, }, W)}{\longrightarrow}}($ Save Game, $S$ ) represents the behavior for input Pause for products which does have the $S$ feature and does not have the $W$ feature for Bowling. Figure 21 shows a different, but equivalent way of modeling the AGM FFSM by composing all three conditional states into one, compose their names and perform a disjunction of their feature constraints.

We can use a sequence of conditional transitions to form a conditional path to generate test-cases. This concept is formalized in the following definition.

Definition 4.1.2. Given a conditional input sequence $\alpha=\left(y_{1}, \ldots, y_{k}\right) \in Y^{*}$, where $y_{i}=\left(x_{i}, \theta_{i}\right) \in Y$ for $1 \leq i \leq k$, there exists a conditional path from conditional state $c_{1}=\left(s_{1}, \varphi_{1}\right)$ to $c_{k+1}$ when there are conditional transitions $t_{i}=\left(c_{i}, y_{i}, o_{i}, c_{i+1}\right) \in \Gamma$, for $1 \leq i \leq k$. A conditional path $\sigma$ is a 4 -tuple $(\nu, \delta, \gamma, \omega)$, where:

1. $\nu=\left(c_{1}, \ldots, c_{k+1}\right) \in C^{*}$ is the conditional state sequence,

2. $\delta=\left(x_{i}, \ldots, x_{k}\right) \in I^{*}$ is the input sequence, 


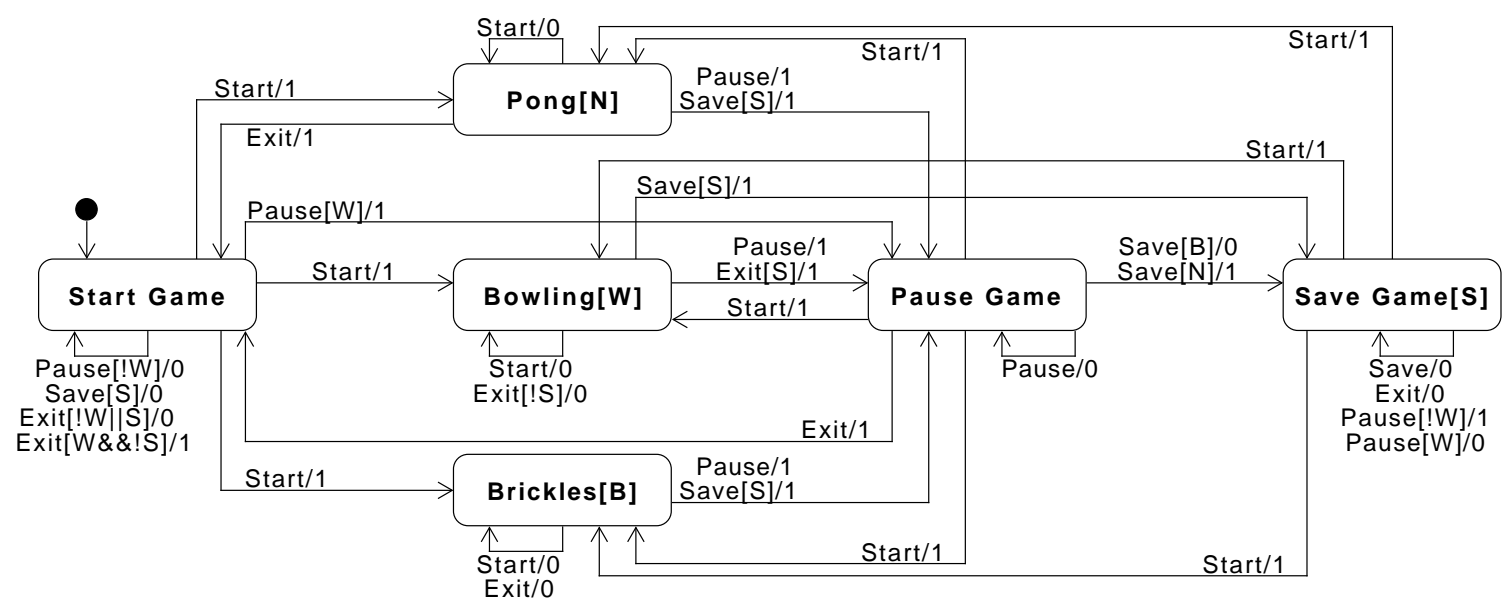

Figure 20 - FFSM for the AGM SPL.

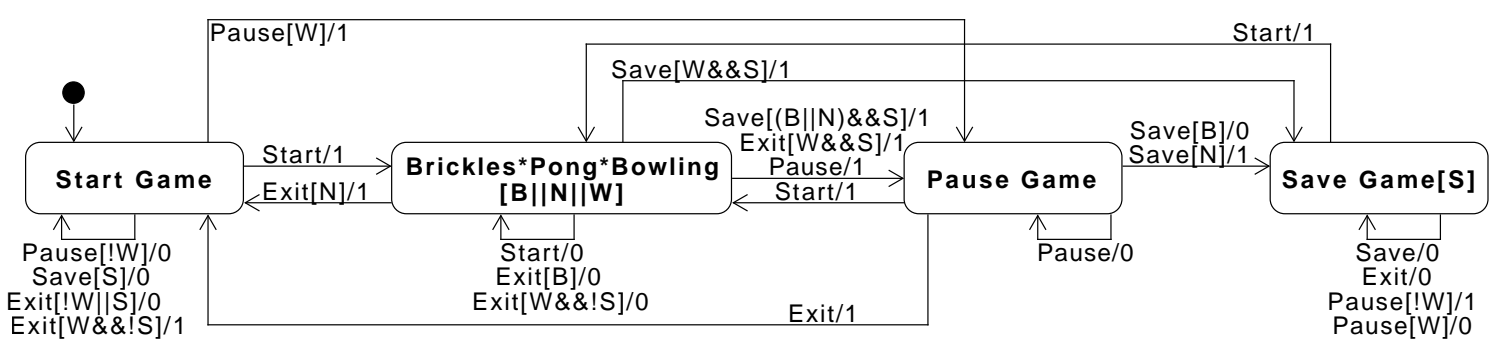

Figure 21 - Alternative FFSM for the AGM SPL.

3. $\gamma=\left(o_{1}, \ldots, o_{k}\right) \in O^{*}$ is the output result,

4. $\omega=\left(\varphi_{1} \wedge \ldots \wedge \varphi_{k+1}\right) \wedge\left(\theta_{1} \wedge \ldots \wedge \theta_{k}\right) \in B(F)$ is the resulting path condition.

A conditional path is valid if there is at least a product configuration $\rho \in \Lambda$ that can satisfy the path condition $\omega$, i.e., $\exists_{\rho \in \Lambda} \bullet \rho \vDash \omega$. Notation $\Theta(c)$ is used to denote the set of all conditional paths that start at conditional state $c \in C$. $\Theta_{F F}$ is used to denote $\Theta\left(c_{0}\right)$.

Example 10. Consider the FFSM of Figure 20. Note that an input sequence $\delta=$ (Start,Pause) of a conditional path $\sigma=$ $(($ Start Game, Brickles, Pause Game $), \delta,(1,1),(B)) \in \Theta_{F F}$, has a path condition $\omega=(B)$ and only two products can satisfy $\omega$, which are $\rho_{5}$ and $\rho_{6}$ (Example 6 ). Thus, $\sigma$ is a valid conditional path.

\subsection{Model Derivation}

To perform model derivation using an FFSM we use a specific feature constraint $\phi$. To use a simplified feature constraint, we first need to define the equivalent relation between feature constraints for a given feature model $F M$. 
Definition 4.2.1. Given an FFSM $F F=\left(F M, C, c_{0}, Y, O, \Gamma\right)$ such that $F M=(F, \chi, \Lambda)$, a feature constraint $\omega_{a}$ is a conditional prefix of $\omega_{b}$ if: (i) there exists configurations that satisfy both feature constraints, i.e. $\exists_{\rho \in \Lambda} \bullet \rho \vDash\left(\omega_{a} \wedge \omega_{b}\right)$; and (ii) the subset of configurations $\Lambda_{a} \subseteq \Lambda$ that satisfies the constraint $\omega_{a}$ is a subset of the subset of configurations $\Lambda_{b} \subseteq \Lambda$ that the constraint $\omega_{b}$ can satisfy, i.e., $\Lambda_{a} \subseteq \Lambda_{b}$.

See Section 6.1.3 to see how conditional prefixes of feature constraints are implemented using the Z3 tool.

Thus, we define a model derivation operator, reminiscent of the operator in (BEOHAR; MOUSAVI, 2014b; BEOHAR; MOUSAVI, 2014a), that is parameterized by feature constraints. Given a feature constraint, the product derivation operator reduces an FFSM into an FSM representing the selection of products.

Definition 4.2.2. Given an FFSM $F F=\left(F M, C, c_{0}, Y, O, \Gamma\right)$, and a product configuration $\rho \in \Lambda$ or a feature constraint $\phi$ equivalent to $\rho$ under $F M$ (Definition 4.2.1), the product derivation operator $\Delta_{\rho}$ can induce an $\operatorname{FSM} \Delta_{\rho}(F F)=\left(S, s_{0}, I, O, T\right)$, where:

1. $S=\{s \mid(s, \varphi) \in C \wedge \rho \vDash(\varphi \wedge \phi)\}$ is the set of states;

2. $s_{0}=s, c_{0}=(s, \varphi) \in C$ is the initial state;

3. $T=\left\{\left(s, x, o, s^{\prime}\right) \mid(s, \varphi) \underset{o}{\stackrel{\left(x, \varphi^{\prime \prime}\right)}{\rightarrow}}\left(s^{\prime}, \varphi^{\prime}\right) \in \Gamma \wedge \rho \vDash\left(\varphi \wedge \varphi^{\prime} \wedge \varphi^{\prime \prime} \wedge \phi\right)\right\}$ is the set of transitions.

Example 11. The set of all valid products of $F F$ is the set of all induced FSMs. Figure 20 shows the FFSM generated for the AGM SPL that can induce six products. Using the feature constraint $\phi=N \wedge \neg S$ the FFSM is projected into an FSM equivalent to the presented in Figure 18.

Abusing the notation we can also reduce an FFSM into a reduced FFSM for a set of products.

Definition 4.2.3. Given a feature constraint $\phi \in B(F)$ and an FFSM $F F=$ $\left(F M, C, c_{0}, Y, O, \Gamma\right)$, if at least one product configuration $\rho \in \Lambda$ satisfies $\phi$, i.e., $\exists_{\rho \in \Lambda} \bullet \rho \vDash \phi$, then the product derivation operator $\nabla_{\phi}$ induces a reduced FFSM $\nabla_{\phi}(F F)=\left(F M^{\prime}, C^{\prime}, c_{0}, Y^{\prime}, O, \Gamma^{\prime}\right)$ with only satisfiable elements.

\subsection{Validation Properties}

To adopt FFSMs as test models, we first need to validate the product-line-based specification with properties used for FSMs. Next, we define the high-level counterparts of the four basic properties, namely, determinism, completeness, initially connectedness, and 
minimality, and show that they coincide with the aforementioned properties for their valid FSM products.

\subsubsection{Determinism}

Definition 4.3.1. An FFSM $F F$ is deterministic if for all conditional states when exists two enabled conditional transitions with the same input for a product (configuration) $\rho$, then both transitions lead to the same state:

$$
\forall \underset{(s, \varphi) \underset{o}{\left(x, \varphi^{\prime}\right)}\left(s^{\prime}, \varphi_{a}\right),(s, \varphi) \underset{o}{\stackrel{\left(x, \varphi^{\prime \prime}\right)}{\longrightarrow}\left(s^{\prime \prime}, \varphi_{b}\right) \in \Gamma}}{ } \bullet \forall_{\rho \in \Lambda} \bullet \rho \not \models\left(\varphi \wedge \varphi^{\prime} \wedge \varphi^{\prime \prime} \wedge \varphi_{a} \wedge \varphi_{b}\right) \vee s^{\prime}=s^{\prime \prime}
$$

Next, we state and prove that an FFSM is deterministic when all its valid product FSMs are deterministic.

Theorem 3. An FFSM $F F$ is deterministic if and only if all derived product FSMs $\Delta_{\phi}(F F)$ are deterministic.

Proof. We break the bi-implication in the thesis into two implications and prove each by contradiction. For the implication from left to right, assume that FFSM $F F$ is deterministic, but there is a derived FSM $\Delta_{\phi}(F F)$ for a product $\rho$ which is non-deterministic; we obtain a contradiction. Let FFSM $F F=\left(F M, C, c_{0}, Y, O, \Gamma\right)$ be deterministic and a derived FSM $\Delta_{\phi}(F F)=\left(S, s_{0}, I, O, T\right)$ be non-deterministic for a product $\rho \in \Lambda$ on state $s \in S$. As $\Delta_{\phi}(F F)$ is non-deterministic, then by the negation of Definition 2.2.3 item 1 there is an input $x \in I$ such that two transitions $\left(s, x, o, s^{\prime}\right),\left(s, x, o, s^{\prime \prime}\right) \in T$ reach different states $s^{\prime} \neq$ $s^{\prime \prime}$. By Definition 4.2.2 item 3 if $\Delta_{\phi}(F F)$ has two transitions $\left(s, x, o, s^{\prime}\right)$ and $\left(s, x, o, s^{\prime \prime}\right)$, then

both were induced from conditional transitions $(s, \varphi) \underset{o}{\stackrel{\left(x, \varphi^{\prime}\right)}{\rightarrow}}\left(s^{\prime}, \varphi_{a}\right),(s, \varphi) \underset{o}{\stackrel{\left(x, \varphi^{\prime \prime}\right)}{\rightarrow}}\left(s^{\prime \prime}, \varphi_{b}\right) \in \Gamma$ of $F F$ and $\rho \vDash\left(\varphi \wedge \varphi^{\prime} \wedge \varphi_{a} \wedge \varphi_{b}\right)$. However, $F F$ is deterministic and by Definition 4.3.1 the condition $\rho \not \models\left(\varphi^{\prime} \wedge \varphi^{\prime \prime} \wedge \varphi_{a} \wedge \varphi_{b}\right) \vee s^{\prime}=s^{\prime \prime}$ holds for all pairs of conditional transitions, which is a contradiction as there is a pair of conditional transitions that the negation of the condition $\rho \vDash\left(\varphi \wedge \varphi^{\prime} \wedge \varphi_{a} \wedge \varphi_{b}\right) \wedge\left(s^{\prime} \neq s^{\prime \prime}\right)$ also holds.

Likewise, for the implication right to left, assume that $\Delta_{\phi}(F F)$ is deterministic for $\rho$, but $F F$ is non-deterministic; we obtain a contradiction. Let $F F=\left(F M, C, c_{0}, Y, O, \Gamma\right)$ be non-deterministic on conditional state $(s, \varphi) \in C, \rho \models \varphi$, and $\Delta_{\phi}(F F)=\left(S, s_{0}, I, O, T\right)$ is deterministic for $\rho$. As $F F$ is non-deterministic, then by the negation of Definition 4.3.1 there is an input $x \in I$ such that two conditional transitions $(s, \varphi) \underset{o}{\stackrel{\left(x, \varphi^{\prime}\right)}{\rightarrow}}\left(s^{\prime}, \varphi_{a}\right),(s, \varphi) \underset{o}{\stackrel{\left(x, \varphi^{\prime \prime}\right)}{\rightarrow}}$ $\left(s^{\prime \prime}, \varphi_{b}\right) \in \Gamma$ are satisfied by $\rho \vDash\left(\varphi \wedge \varphi^{\prime} \wedge \varphi_{a} \wedge \varphi_{b}\right)$ and reach different states $s^{\prime} \neq s^{\prime \prime}$. As $\rho \vDash \phi$ and by Definition 4.2.2 item 3 each transition of $F F$ that satisfies $\phi$ is induced to $\Delta_{\phi}(F F)$, thus $\left(s, x, o, s^{\prime}\right),\left(s, x, o, s^{\prime \prime}\right) \in T$. However, $\Delta_{\phi}(F F)$ is deterministic and by Definition 2.2.3 item 1 the condition $s^{\prime}=s^{\prime \prime}$ is true for all pairs of transitions $\left(s, x, o, s^{\prime}\right),\left(s, x, o, s^{\prime \prime}\right) \in T$, 
which is a contradiction as there is a pair of transitions $\left(s, x, o, s^{\prime}\right),\left(s, x, o, s^{\prime \prime}\right) \in T$ such $\left(s^{\prime} \neq s^{\prime \prime}\right)$.

\subsubsection{Complete}

Definition 4.3.2. An FFSM FF is complete if for all conditional states in a product there is an outgoing valid transition for each and every input:

$$
\forall_{(s, \varphi) \in C} \bullet \forall_{\rho \in \Lambda} \bullet \forall_{x \in I} \bullet \rho \not \models \varphi \vee \exists \underset{(s, \varphi) \underset{o}{\left(x, \varphi^{\prime \prime}\right)}\left(s^{\prime}, \varphi^{\prime}\right) \in \Gamma}{ } \bullet \rho \models \varphi^{\prime} \wedge \varphi^{\prime \prime}
$$

Next, we state and prove that an FFSM is complete when all its valid product FSMs are complete.

Theorem 4. An FFSM is complete if and only if all derived product FSMs are complete.

Proof. We break the bi-implication in the thesis into two implications and prove each by contradiction. For the implication left to right, assume that FFSM FF is complete, but there is a derived FSM $\Delta_{\phi}(F F)$ for a product $\rho$ which is non-complete; we obtain a contradiction. Let FFSM $F F=\left(F M, C, c_{0}, Y, O, \Gamma\right)$ be complete and a derived FSM $\Delta_{\phi}(F F)=\left(S, s_{0}, I, O, T\right)$ be non-complete for a product $\rho \in \Lambda$ on state $s \in S$ for input $x \in I$. As $\Delta_{\phi}(F F)$ is non-complete, then, by the negation of Definition 2.2.3 item 2 there is no transition $\left(s, x, o, s^{\prime}\right) \in T$ on $s$ with input $x$. By Definition 4.3.2 if $F F$ is complete, then for all products $\rho \in \Lambda$ that satisfies a conditional state $(s, \varphi) \in C \wedge \rho \models \varphi$ and for all inputs $x \in I$ there are conditional transitions $(s, \varphi) \underset{o}{\stackrel{\left(x, \varphi^{\prime \prime}\right)}{\rightarrow}}\left(s^{\prime}, \varphi^{\prime}\right) \in \Gamma$ such $\rho \models \varphi^{\prime} \wedge \varphi^{\prime \prime}$. However, by Definition 4.2.2 item 3 every conditional transition $(s, \varphi) \underset{o}{\stackrel{\left(x, \varphi^{\prime \prime}\right)}{\rightarrow}}\left(s^{\prime}, \varphi^{\prime}\right) \in \Gamma$ in $F F$ that satisfies $\rho \vDash \phi$ induces a transition $\left(s, x, o, s^{\prime}\right) \in T$ in $\Delta_{\phi}(F F)$, which is a contradiction as $\Delta_{\phi}(F F)$ does not have a transition $\left(s, x, o, s^{\prime}\right) \in T$ on state $s$ for input $x$.

Likewise, for the implication right to left, assume that $\Delta_{\phi}(F F)$ is complete for $\rho$, but $F F$ is non-complete; we obtain a contradiction. Let $F F=\left(F M, C, c_{0}, Y, O, \Gamma\right)$ be non-complete on conditional state $(s, \varphi) \in C$ for input $x \in I, \rho \models \varphi$, and $\Delta_{\phi}(F F)=$ $\left(S, s_{0}, I, O, T\right)$ is complete for $\rho$. As $F F$ is non-complete, then by the negation of Definition 4.3.2 on conditional state $(s, \varphi) \in C$ there is no conditional transition $(s, \varphi) \underset{o}{\stackrel{\left(x, \varphi^{\prime \prime}\right)}{\rightarrow}}\left(s^{\prime}, \varphi^{\prime}\right) \in \Gamma$ with input $x \in I$ for $F F$, or it exists but is not satisfied $\rho \not \models \varphi^{\prime} \wedge \varphi^{\prime \prime}$. By Definition 4.2.2 item 3 if a conditional transition $(s, \varphi) \underset{o}{\stackrel{\left(x, \varphi^{\prime \prime}\right)}{\rightarrow}}\left(s^{\prime}, \varphi^{\prime}\right)$ does not exist in $F F$, or it exists but $\rho \not \models \varphi^{\prime} \wedge \varphi^{\prime \prime}$, then there is no transition $\left(s, x, o, s^{\prime}\right) \in T$ induced in $\Delta_{\phi}(F F)$. However, $\Delta_{\phi}(F F)$ is complete and by Definition 2.2.3 item 2 for all states $s \in S$ and for all inputs $x \in I$ there are transitions $\left(s, x, o, s^{\prime}\right) \in T$, which is a contradiction as there is no transition $\left(s, x, o, s^{\prime}\right) \in T$ in $\Delta_{\phi}(F F)$ for state $s$ and input $x$. 


\subsubsection{Initially Connected}

Definition 4.3.3. An FFSM FF is initially connected if there exist conditional path starting on the initial conditional state to every conditional state for every satisfiable product:

$$
\forall_{c=(s, \varphi) \in C} \bullet \forall_{\rho \in \Lambda} \bullet \rho \models \varphi \Longrightarrow \exists_{\left(\left(c_{0}, \ldots, c\right), \delta, \gamma, \omega\right) \in \Theta_{F F}} \bullet \rho \vDash \omega
$$

Next, we state and prove that an FFSM is initially connected when all its valid product FSMs are initially connected.

Theorem 5. An FFSM is initially connected if and only if all derived product FSMs are initially connected.

Proof. We break the bi-implication in the thesis into two implications and prove each by contradiction. For the implication left to right, assume that FFSM FF is initially connected, but there is a derived FSM $\Delta_{\phi}(F F)$ for a product $\rho$ which is non-initially connected; we obtain a contradiction. Let FFSM $F F=\left(F M, C, c_{0}, Y, O, \Gamma\right)$ be initially connected and a derived FSM $\Delta_{\phi}(F F)=\left(S, s_{0}, I, O, T\right)$ be non-initially connected for a product $\rho \in \Lambda$ on state $s_{k} \in S$. As $\Delta_{\phi}(F F)$ is non-initially connected, then, by the negation of Definition 2.2.3 item 3 there is no path $v \in \Omega_{\Delta_{\phi}(F F)}$ to $s_{k}$ from the initial state $s_{0}$. By Definition 4.3.3 if $F F$ is initially connected, then there is a path $\sigma_{k} \in \Theta_{F F}$ to every conditional state $\left(s_{k}, \varphi_{k}\right) \in C$ from the initial conditional state $c_{0}$, and $\rho$ satisfies the path condition $\omega$. However, by Definition 4.1.2 every conditional transition $\left(s_{i}, \varphi_{i}\right) \underset{o}{\stackrel{\left(x_{i}, \varphi_{i}^{\prime}\right)}{\rightarrow}}\left(s_{i+1}, \varphi_{i+1}\right) \in \Gamma$, $0 \leq i \leq k$ forms a path to reach $\left(s_{k}, \varphi_{k}\right)$ which is satisfied by $\rho$. As $\rho \vDash \phi$, and by Definition 4.2.2 item 3 every conditional transition $\left(s_{i}, \varphi_{i}\right) \underset{o}{\stackrel{\left(x_{i}, \varphi_{i}^{\prime}\right)}{\rightarrow}}\left(s_{i+1}, \varphi_{i+1}\right) \in \Gamma$ is induced to $\left(s_{i}, x_{i}, o, s_{i+1}\right) \in T$ that forms a path to reach $s_{k}$, which is a contradiction as there is no path for $v \in \Omega_{\Delta_{\phi}(F F)}$ to reach state $s_{k}$.

Likewise, for the implication right to left, assume that $\Delta_{\phi}(F F)$ is initially connected for $\rho$, but $F F$ is non-initially connected; we obtain a contradiction. Let $F F=\left(F M, C, c_{0}, Y, O, \Gamma\right)$ be non-initially connected on conditional state $(s, \varphi) \in C$, $\rho \models \varphi$, and $\Delta_{\phi}(F F)=\left(S, s_{0}, I, O, T\right)$ is initially connected for $\rho$. As $F F$ is non-initially connected, then by the negation of Definition 4.3.3 there is no path $\sigma \in \Theta_{F F}$ to reach $\left(s_{k}, \varphi_{k}\right)$ from the initial conditional state $c_{0}$. By Definition 2.2.3 item 3 if $\Delta_{\phi}(F F)$ is initially connected, then there is a path $v \in \Omega_{\Delta_{\phi}(F F)}$ to reach every state $s \in S$ from the initial state $s_{0}$. As $\rho \vDash \phi$, and by Definition 4.2.2 item 3 every transition $\left(s_{i}, x_{i}, o, s_{i+1}\right) \in T$ was induced from a conditional transition $\left(s_{i}, \varphi_{i}\right) \underset{o}{\stackrel{\left(x_{i}, \varphi_{i}^{\prime}\right)}{\rightarrow}}\left(s_{i+1}, \varphi_{i+1}\right) \in \Gamma$ and $\rho \vDash \varphi_{i} \wedge \varphi_{i}^{\prime} \wedge \varphi_{i+1}$ that forms a path to reach $\left(s_{k}, \varphi_{k}\right)$, which is a contradiction as there is no path $\sigma \in \Theta_{F F}$ to reach $\left(s_{k}, \varphi_{k}\right)$. 


\subsubsection{Minimal}

Definition 4.3.4. An FFSM $F F$ is minimal if for all pairs of conditional states of all satisfiable products there are separating sequences that distinguish both conditional states:

$$
\begin{array}{r}
\forall_{c_{a}=\left(s_{a}, \varphi_{a}\right), c_{b}=\left(s_{b}, \varphi_{b}\right) \in C} \bullet \forall_{\rho \in \Lambda} \bullet \rho \vDash \varphi_{a} \wedge \varphi_{b} \Rightarrow \\
\exists_{\left(\left(c_{a}, \ldots, c_{a^{\prime}}\right), \delta, \gamma_{a}, \omega_{a}\right) \in \Theta\left(c_{a}\right),\left(\left(c_{b}, \ldots, c_{b^{\prime}}\right), \delta, \gamma_{b}, \omega_{b}\right) \in \Theta\left(c_{b}\right)} \bullet \gamma_{a} \neq \gamma_{b} \wedge \rho \vDash\left(\omega_{a} \wedge \omega_{b}\right)
\end{array}
$$

Next, we state and prove that an FFSM is minimal when all its valid product FSMs are minimal.

Theorem 6. An FFSM is minimal if and only if all derived product FSMs are minimal.

Proof. We break the bi-implication in the thesis into two implications and prove each by contradiction. For the implication left to right, assume that FFSM FF is minimal, but there is a derived FSM $\Delta_{\phi}(F F)$ for a product $\rho$ which is non-minimal; we obtain a contradiction. Let FFSM $F F=\left(F M, C, c_{0}, Y, O, \Gamma\right)$ be minimal and a derived FSM $\Delta_{\phi}(F F)=\left(S, s_{0}, I, O, T\right)$ be non-minimal for a product $\rho \in \Lambda$ on states $s_{a}, s_{b} \in S$. As $\Delta_{\phi}(F F)$ is non-minimal, then, by the negation of Definition 2.2.3 item 4 there is no common input sequence $\alpha \in I^{*}$ of two paths $v_{a} \in \Omega\left(s_{a}\right), v_{b} \in \Omega\left(s_{b}\right)$ that distinguish states $s_{a}$ and $s_{b}$. By Definition 4.3.4 if FF is minimal, then for every pair of conditional states $c_{a}=\left(s_{a_{0}}, \varphi_{a_{0}}\right), c_{b}=\left(s_{b_{0}}, \varphi_{b_{0}}\right) \in C$ and for all products $\rho \in \Lambda$ that satisfy the condition $\varphi_{a_{0}} \wedge \varphi_{b_{0}}$ there are two paths with a common a separating sequence $\delta \in Y^{*}$ and $\rho$ also satisfies both path conditions $\omega_{a} \wedge \omega_{b}$. However, by Definition 4.1.2 every pair of conditional transitions $\left(s_{a_{i}}, \varphi_{a_{i}}\right) \underset{o}{\stackrel{\left(x_{i}, \varphi_{i}^{\prime}\right)}{\rightarrow}}\left(s_{a_{i+1}}, \varphi_{a_{i+1}}\right),\left(s_{b_{i}}, \varphi_{b_{i}}\right) \underset{o^{\prime}}{\stackrel{\left(x_{i}, \varphi_{i}^{\prime \prime}\right)}{\rightarrow}}\left(s_{b_{i+1}}, \varphi_{b_{i+1}}\right) \in \Gamma, 0 \leq i \leq k$ of the separating sequence $\delta$ is satisfied by $\rho$. As $\rho \vDash \phi$, and by Definition 4.2 .2 item 3 every pair of conditional transitions $\left(s_{a_{i}}, \varphi_{a_{i}}\right) \underset{o}{\stackrel{\left(x_{i}, \varphi_{i}^{\prime}\right)}{\rightarrow}}\left(s_{a_{i+1}}, \varphi_{a_{i+1}}\right),\left(s_{b_{i}}, \varphi_{b_{i}}\right) \underset{o^{\prime}}{\left(x_{i}, \varphi_{i}^{\prime \prime}\right)}\left(s_{b_{i+1}}, \varphi_{b_{i+1}}\right) \in \Gamma$ is induced to $\left(s_{a_{i}}, x_{i}, o, s_{a_{i+1}}\right),\left(s_{b_{i}}, x_{i}, o^{\prime}, s_{b_{i+1}}\right) \in T$ in $\Delta_{\phi}(F F)$ that distinguishes $s_{a}$ and $s_{b}$, which is a contradiction as there is no separating sequence $\alpha \in I^{*}$ for states $s_{a}$ and $s_{b}$.

Likewise, for the implication right to left, assume that $\Delta_{\phi}(F F)$ is minimal for $\rho$, but $F F$ is non-minimal; we obtain a contradiction. Let $F F=\left(F M, C, c_{0}, Y, O, \Gamma\right)$ be non-minimal on conditional state $c_{a}=\left(s_{a_{0}}, \varphi_{a_{0}}\right), c_{b}=\left(s_{b_{0}}, \varphi_{b_{0}}\right) \in C, \rho \models \varphi$, and $\Delta_{\phi}(F F)=\left(S, s_{0}, I, O, T\right)$ is minimal for $\rho$. As $F F$ is non-minimal, then by the negation of Definition 4.3.4 there is no common input sequence $\delta \in Y^{*}$ that distinguish conditional states $c_{a}$ and $c_{b}$. By Definition 2.2.3 item 4 if $\Delta_{\phi}(F F)$ is minimal, then there are two paths with a common separating sequence $\alpha \in I^{*}$ for every pair of states $s_{a}$ and $s_{b}$. As $\rho \vDash \phi$, and by Definition 4.2.2 item 3 every pair of transitions $\left(s_{a_{i}}, x_{i}, o, s_{a_{i+1}}\right),\left(s_{b_{i}}, x_{i}, o^{\prime}, s_{b_{i+1}}\right) \in T$ were induced from $\left(s_{a_{i}}, \varphi_{a_{i}}\right) \underset{o}{\stackrel{\left(x_{i}, \varphi_{i}^{\prime}\right)}{\rightarrow}}\left(s_{a_{i+1}}, \varphi_{a_{i+1}}\right),\left(s_{b_{i}}, \varphi_{b_{i}}\right) \underset{o^{\prime}}{\stackrel{\left(x_{i}, \varphi_{i}^{\prime \prime}\right)}{\rightarrow}}\left(s_{b_{i+1}}, \varphi_{b_{i+1}}\right) \in \Gamma$ and $\rho$ satisfies both conditional paths that distinguishes $c_{a}$ and $c_{b}$, which is a contradiction as there is no separating sequence $\delta \in Y^{*}$ for $c_{a}$ and $c_{b}$. 


\subsection{Test Design}

In this section, we define configurable test-cases and how they can be instantiated to derive specific test-suites, then we extend basic test definitions used in FSM-based test-case generation for state coverage, transition coverage, and the HSI method (LUO et al., 1994) for FFSMs. Only FFSMs specifications that are deterministic, initially connected, and minimal are used for test-case design.

\subsubsection{Configurable Test-suites}

To generate conditional test-cases, we use sequences of inputs that are valid in at least one product configuration. A configurable test-suite, also defined below, is a set of conditional tests.

Definition 4.4.1. Given an FFSM $F F=\left(F M, C, c_{0}, Y, O, \Gamma\right)$ such that $F M=(F, \chi, \Lambda)$, a conditional test-case (or simply a conditional test) of $F F$ is a tuple $(\delta, \omega) \in I^{*} \times B(F)$, where $\delta$ is an input sequence of a valid conditional path $\left(\left(c_{0}, \ldots, c\right), \delta, \gamma, \omega\right) \in \Theta_{F F}$, and $\omega$ is the feature constraint of the path. A configurable test-suite $C T S \subseteq \mathcal{P}\left(I^{*} \times B(F)\right)$ of $F F$ is a finite set of conditional tests of $F F$.

To determine whether a conditional test $\left(\delta_{a}, \omega_{a}\right)$ is a conditional prefix of another conditional test $\left(\delta_{b}, \omega_{b}\right)$ we use the feature model to compare the configurations satisfied by each feature constraint.

Definition 4.4.2. Given an $F F=\left(F M, C, c_{0}, Y, O, \Gamma\right)$ such that $F M=(F, \chi, \Lambda)$, a conditional test $\left(\delta_{a}, \omega_{a}\right)$ is a conditional prefix of $\left(\delta_{b}, \omega_{b}\right)$ if: (i) $\delta_{a}$ is a prefix of $\delta_{b}$; (ii) there exist configurations that satisfy both feature constraints, i.e., $\exists_{\rho \in \Lambda} \bullet \rho \vDash\left(\omega_{a} \wedge \omega_{b}\right)$; and (iii) $\Lambda_{a} \subseteq \Lambda_{b}$, where $\Lambda_{a} \Lambda_{b} \subseteq \Lambda$ are the subsets of configurations satisfying $\omega_{a}$ and $\omega_{b}$, respectively.

We denote by $\operatorname{cpref}(\delta, \omega)$ the set of prefixes of $(\delta, \omega)$, and cpref $(C T S)$ for the prefixes of all tests in a configurable test-suite. Moreover, given two conditional tests $\left(\delta_{a}, \omega_{a}\right)$ and $\left(\delta_{b}, \omega_{b}\right)$, if $\delta_{a}=\delta_{b}$, then they can be merged into a conditional test $\left(\delta_{a},\left(\omega_{a} \vee \omega_{b}\right)\right)$. Please see Section 6.1.3 for more details about the prefix check using the Z3 tool.

\subsubsection{Test-case Derivation}

The product derivation operator $\Delta_{\phi}$ induces an FSM from a given FFSM and a given feature constraint $\phi$ (Definition 4.2.2). Similarly, we define the derivation of testsuites from a configurable test-suite of an FFSM. The feature constraint $\phi$ is able to filter/prune test-cases from the test-suite in the same way a configuration model is used for test models. 
Definition 4.4.3. Let $F F=\left(F M, C, c_{0}, Y, O, \Gamma\right)$ be an FFSM, a configurable test-suite $C T S$ for $F F$, and a product configuration $\rho \in \Lambda$. The derivation operator $\Delta_{\rho}$ induces a test-suite $\Delta_{\rho}(C T S) \subseteq \mathcal{P}\left(I^{*}\right)$ for an FSM, where:

$$
\Delta_{\rho}(C T S)=\{\delta \mid(\delta, \omega) \in C T S \wedge \rho \vDash \omega\}
$$

\subsubsection{State Coverage}

To define the state coverage criteria for FFSMs we need conditional paths to reach conditional states on every satisfiable product. Assuming that a conditional state is satisfiable by two product configurations it might be necessary to find two distinct paths to reach and cover the state.

Definition 4.4.4. Given an FFSM $F F=\left(F M, C, c_{0}, Y, O, \Gamma\right)$ and a conditional state $c=(s, \varphi) \in C$, the set $C T S \subseteq \mathcal{P}\left(I^{*} \times B(F)\right)$ covers $c$ for the state coverage criteria if for all product configurations that satisfy $\varphi$ there is a valid conditional path $\left(\left(c_{0}, \ldots, c\right), \delta, \gamma, \omega\right) \in$ $\Theta_{F F}$ to reach $c$ and $(\delta, \omega) \in C T S$. The set $C T S$ is a configurable state cover test-suite if it covers every conditional state of $F F$ :

$$
\forall_{c=(s, \varphi) \in C} \bullet \forall_{\rho \in \Lambda} \bullet \rho \vDash \varphi \Longrightarrow \exists_{\left(\left(c_{0}, \ldots, c\right), \delta, \gamma, \omega\right) \in \Theta_{F F}} \bullet \rho \vDash(\omega \wedge \varphi) \wedge(\delta, \omega) \in C T S
$$

Example 12. The FFSM FF presented in Figure 20 was abstracted to ease visualization, and is presented in Figure 22. State names were substituted for numbers: Start Game as state 1, Pong Game as state 2, Bowling Game as state 3, Brickles Game as state 4, Pause Game as state 5, and Save Game as state 6. For inputs: Start as input a, Pause as input $b$, Exit as input $c$, and Save as input $d$. Dashed transitions are part of the sets of tests required to cover all states, which are: to cover 1 we use the empty sequence $\{(\varepsilon,($ true $))\}$, for $2\{(a,(N))\}$, for $3\{(a,(W))\}$, for $4\{(a,(B))\}$, for $5\{(a b,(N)),(b,(W)),(a b,(B))\}$, and for $6\{(a b d,(N \wedge S)),(a d,(W \wedge S)),(a b d,(B \wedge S))\}$.

Algorithm 1 generates a configurable state cover set. We identify conditional paths using a breadth-first search looking for different paths combinations excluding self-loops transitions and those that create a loop in the current path resulting in paths smaller than the number of states.

Example 13. Figure 23 presents a conditional testing tree generated of the conditional state cover set CTS for FF. The tree is built in the following order: left-right and top-bottom. Every arrow is labeled with the input of the transition and an equivalent accumulated feature constraint of the path. Conditional states are marked with three node shapes: circle for covered conditional states; triangle for partially covered conditional states; 


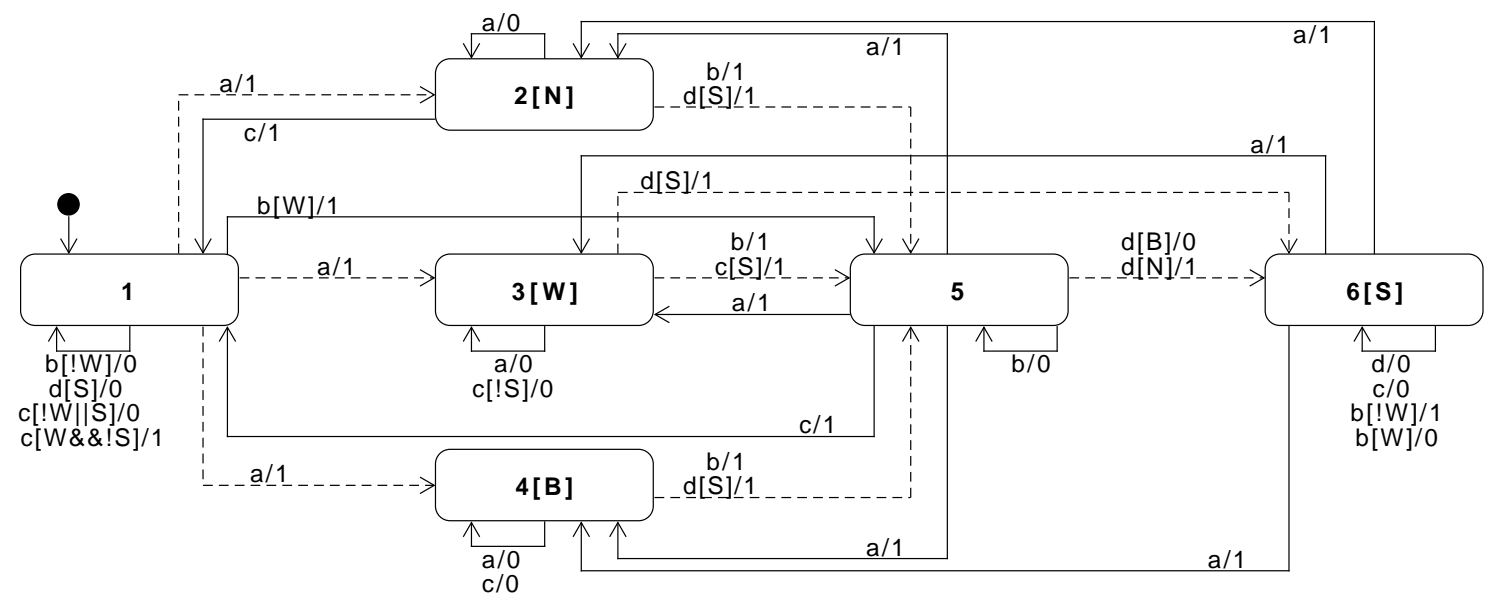

Figure 22 - Abstracted FFSM of AGM.

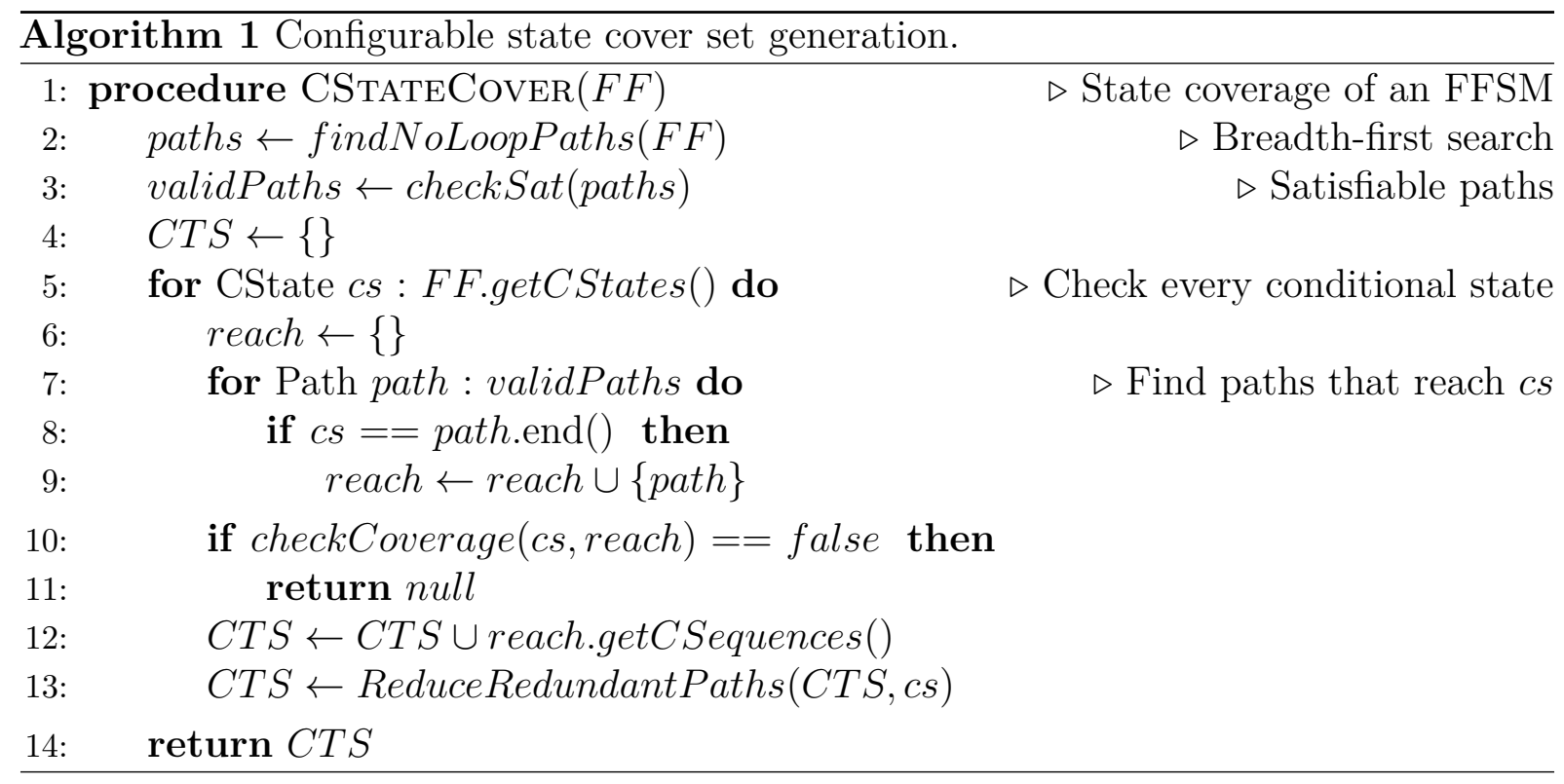

and square for invalid paths that do not reach the conditional state. Triangle conditional states are marked as visited but not covered at that point of execution, then, other paths are required to cover the remaining configurations. Thus, after merging conditional tests, substitute long feature constraints with smaller equivalent ones, and removing conditional test prefixes the resulting set cpref $(C T S)=(\{(a,($ true $)),(b,(W)),(a b,(N \vee$ $B)),(a d,(W \wedge S)),(a b d,((B \wedge S) \vee(N \wedge S)))\})$ is a conditional state cover set for $F F$. Note that this is not the only solution as there are several different paths that could be used to cover a condition state. Applying the derivation operator for the product configuration $\rho_{3}=(\ldots N \wedge \neg S \ldots)$ on the FFSM $F F$ (Figure 22) we obtain the FSM $\Delta_{\rho_{3}}(F F)$ (Figure 7 ). Applying the same operator on $C T S$ (Definition 4.4.3) we obtain a transition cover set $\Delta_{\rho_{3}}(C T S)=\operatorname{pref}(\{a b\})$ that is the same state cover set presented in Example 2.

Next, we state and prove that a configurable state cover set is a state cover set for 


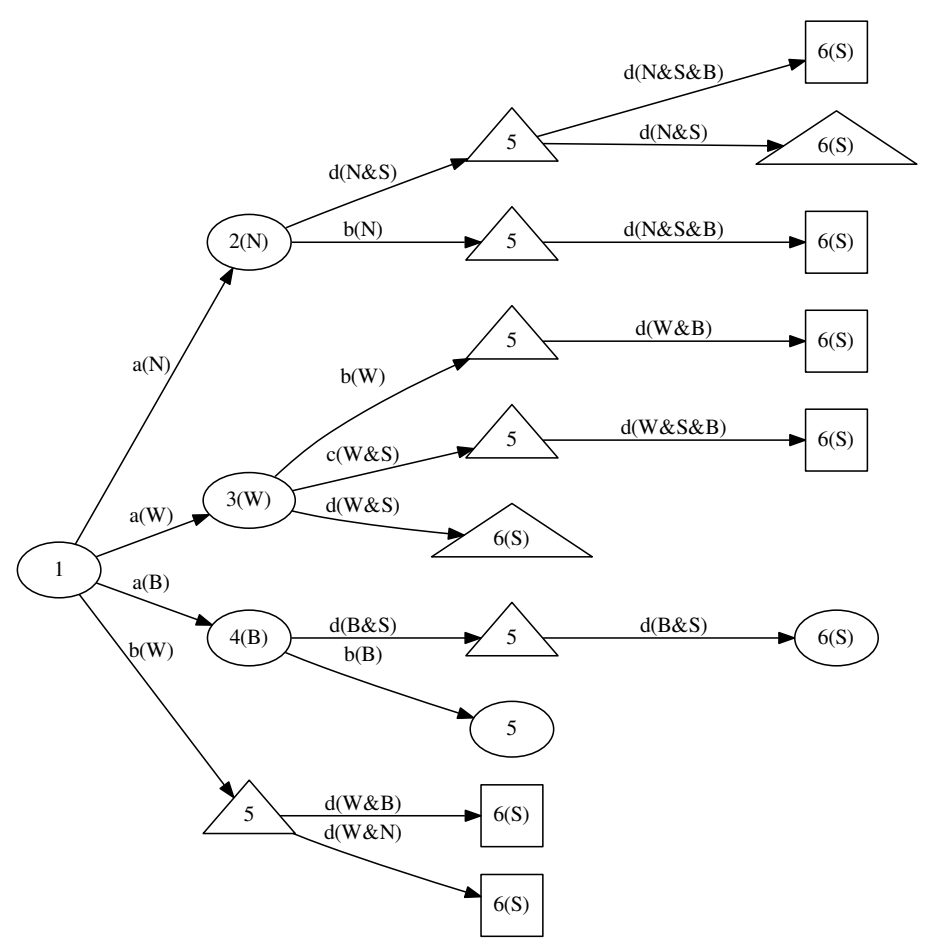

Figure 23 - Conditional state tree for AGM.

all its valid product FSMs.

Theorem 7. If the test-suite $C T S \subseteq \mathcal{P}\left(I^{*} \times B(F)\right)$ is a configurable state cover for an FFSM $F F$, then $C T S$ contains a state cover set $\Delta_{\rho}(C T S) \subseteq \mathcal{P}\left(I^{*}\right)$ for all derived product FSMs $\Delta_{\rho}(F F)$.

Proof. We prove the implication by contradiction. Let the set $C T S \subseteq \mathcal{P}\left(I^{*} \times B(F)\right)$ be a configurable state cover for an FFSM $F F=\left(F M, C, c_{0}, Y, O, \Gamma\right)$ and assume that the set $\Delta_{\rho}(C T S) \subseteq \mathcal{P}\left(I^{*}\right)$ does not cover a state $s \in S$ for a product configuration $\rho$ under a derived FSM $\Delta_{\rho}(F F)=\left(S, s_{0}, I, O, T\right)$. By Definition 4.4.4 for every product configuration $\rho \in \Lambda$ that can be satisfied by the feature constraint of a conditional state $c=(s, \varphi) \in C$ there exist a valid conditional path $\left(\left(c_{0}, \ldots, c\right), \delta, \gamma, \omega\right) \in \Theta_{F F}$ that reaches $c$. By Definition 4.4.3 the conditional input sequence $(\delta, \omega) \in C T S$ derives an input sequence $\delta \in \Delta_{\rho}(C T S)$ for $\rho$ that reaches $s$, which leads to a contradiction as $\delta \notin \Delta_{\rho}(C T S)$.

\subsubsection{Transition Coverage}

To define the transition coverage criteria for FFSMs we use valid conditional paths to reach conditional source states of conditional transitions for all valid products, and then include each and every outgoing transition. 
Definition 4.4.5. Given an FFSM $F F=\left(F M, C, c_{0}, Y, O, \Gamma\right)$, a configurable state cover $C T S \subseteq \mathcal{P}\left(I^{*} \times B(F)\right)$ for $F F$ (Definition 4.4.4), and a conditional transition $t=\left(c_{a},(x, \varphi), o,\left(s_{b}, \omega_{b}\right)\right) \in \Gamma$, the test-suite CTS covers $t$ if for all conditional tests $(\delta, \omega) \in C T S$ that reach $c_{a}$ such that there is a product configuration $\rho \in \Lambda$ that satisfies $\varphi_{t}=\left(\omega \wedge \varphi \wedge \omega_{b}\right)$, then $\left(\delta x, \varphi_{t}\right) \in C T S$. The set CTS is a configurable transition cover test-suite if it covers every conditional transition of $F F$ :

$$
\forall_{t \in \Gamma} \bullet \forall_{(\delta, \omega) \in C T S} \bullet \exists_{\left(\left(c_{0}, \ldots, c_{a}\right), \delta, \gamma, \omega\right) \in \Theta_{F F}} \bullet \exists_{\rho \in \Lambda} \bullet \rho \vDash \varphi_{t} \Longrightarrow\left(\delta x, \varphi_{t}\right) \in C T S
$$

Example 14. Figure 24 presents a conditional testing tree generated of the conditional transition cover set $C T S$ for $F F$. The tree was extended from the state coverage tree of Example 13. Filled conditional states were already visited in the current path, thus they are leafs of the tree. Invalid paths were not shown to save space. Normal arrows represent covered transitions and dashed arrows represent partially covered transitions (not covered for all product configurations) at that point of execution. Conditional states that contain partially covered transitions spawn the remaining transitions that were left to be covered until all of them become covered. For example, on conditional states 5 and 6 , only a few transitions are covered every time that they appear, or they just increment the number of product configurations covered. Thus, after merging conditional tests, substitute long feature constraints with smaller equivalent ones, and removing conditional test prefixes the resulting set is a conditional transition cover set for $F F$ : cpref $(C T S)=(\{(b,($ true $)),(d,(S)),(c,($ true $)),($ ac,$(N \vee B \vee(W \wedge \neg S))),($ aa,$($ true $))$,

$$
\begin{aligned}
& (b c,(W)),(b a,(W)),(b b,(W)),(a d a,(S)),(a d c,(S)),(a d b,(S)),(a d d,(S)), \\
& (a b a,(\text { true })),(a b c,(\text { true })),(a b b,(\text { true })),(a c c,(W \wedge S)),(a c b,(W \wedge S)), \\
& (a d d b,((N \vee B) \wedge S \wedge \neg W)),(a d d c,((N \vee B) \wedge S)),(\text { addd },((N \vee B) \wedge S)), \\
& (a d d a,((N \vee B) \wedge S))\})
\end{aligned}
$$

Applying the derivation operator for the product configuration $\rho_{3}=(\ldots N \wedge \neg S \ldots)$ on the FFSM $F F$ we obtain the FSM $\Delta_{\rho_{3}}(F F)$. Applying the same operator on $C T S$ (Definition 4.4.3) we obtain a transition cover set $\Delta_{\rho_{3}}(C T S)=\operatorname{pref}(\{b, c, a c, a a, a b a, a b b, a b c\})$ that is the same transition cover set presented in Figure 8.

Algorithm 2 generates a configurable transition cover set. We reuse a subset of the configurable state cover set to reach the source state of a conditional transition, and then we concatenate the conditional transition to each reachable path, check whether if it forms a valid conditional path, and finally add new conditional tests in CTS.

To state and prove that a configurable transition cover set is a transition cover set for all its valid product FSMs, first we redefine a transition cover set for an FSM, then, we move to FFSMs. 


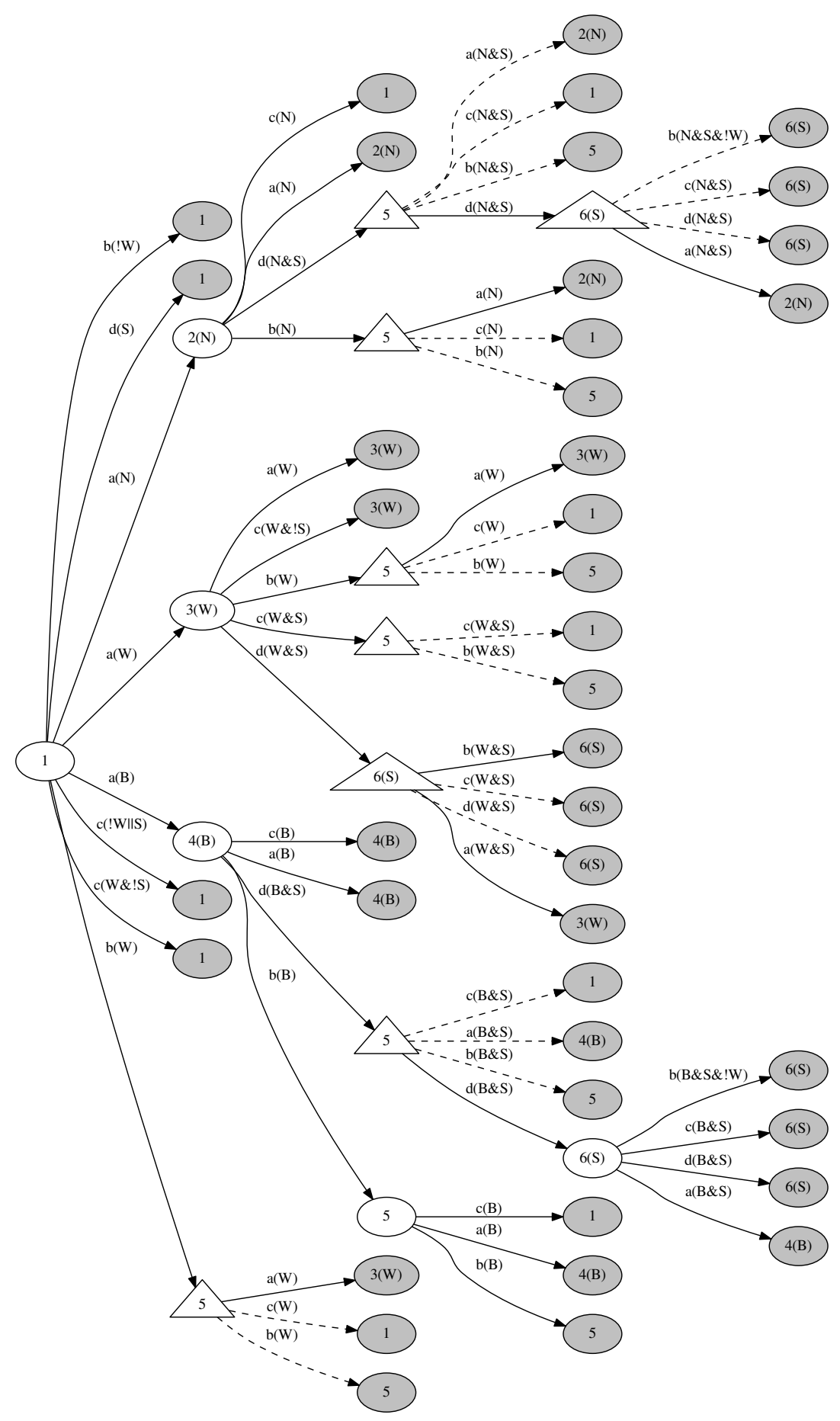

Figure 24 - Conditional transition tree for AGM. 


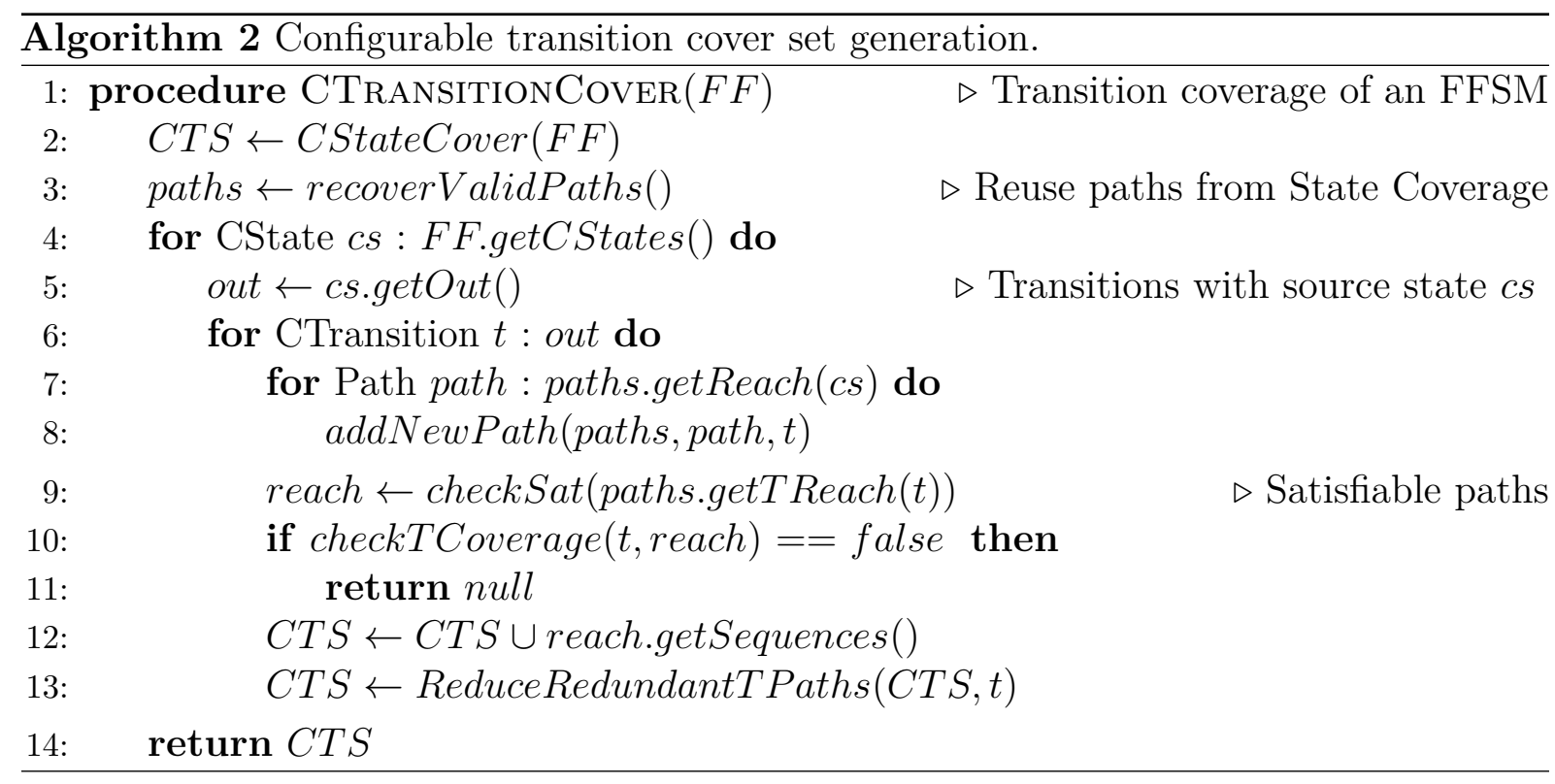

Theorem 8. If the set $C T S \subseteq \mathcal{P}\left(I^{*} \times B(F)\right)$ is a configurable transition cover for an FFSM $F F$, then $C T S$ contains a transition cover set $\Delta_{\rho}(C T S) \subseteq \mathcal{P}\left(I^{*}\right)$ for all derived product FSMs $\Delta_{\rho}(F F)$.

Proof. We prove the implication by contradiction. Let the set $C T S \subseteq \mathcal{P}\left(I^{*} \times B(F)\right)$ be a configurable transition cover for an FFSM $F F=\left(F M, C, c_{0}, Y, O, \Gamma\right)$ and assume that the set $\Delta_{\rho}(C T S) \subseteq \mathcal{P}\left(I^{*}\right)$ does not cover a transition $t=\left(s_{a}, x, o, s_{b}\right) \in T$ for a product configuration $\rho$ under a derived $\operatorname{FSM} \Delta_{\rho}(F F)=\left(S, s_{0}, I, O, T\right)$. By Definition 4.4.4 and 4.4.5 for every product configuration $\rho \in \Lambda$ that can be satisfied by the feature constraint of a conditional state $c_{a}=\left(s_{a}, \varphi_{a}\right) \in C$ there exist a valid conditional path $\left(\left(c_{0}, \ldots, c_{a}\right), \delta, \gamma, \omega\right) \in \Theta_{F F}$ that reaches $c_{a}$, and a transition $t=\left(c_{a},(x, \varphi), o,\left(s_{b}, \varphi_{b}\right)\right) \in \Gamma$ such that $\left(\delta x,\left(\omega \wedge \varphi \wedge \varphi_{b}\right)\right) \in C T S$. By Definition 4.4.3 the conditional test $(\delta x,(\omega \wedge \varphi \wedge$ $\left.\left.\varphi_{b}\right)\right) \in C T S$ derives an input sequence $\delta x \in \Delta_{\rho}(C T S)$ for $\rho$ that reaches $s_{a}$ concatenated with $x$, which leads to a contradiction as $\delta x \notin \Delta_{\rho}(C T S)$.

\subsubsection{Full Fault Coverage}

To extend the HSI method for FFSMs we are going to use two sets: (i) a configurable transition cover set $C T S$; and (ii) a configurable characterizing set $C W$ to distinguish conditional states. We have defined the configurable transition cover set $C T S$ in Definition 4.4.5. Now we need to define parametrized separating sets, define a configurable characterizing set, and then build conditional HSI sets for state identification of FFSMs.

\section{Parametrized Separating Set}

To distinguish a pair of states of an FSM we only need one separating sequence. However, to distinguish a pair of conditional states of an FFSM we need a set of separating 
sequences.

Definition 4.4.6. Let $c_{a}=\left(s_{a}, \varphi_{a}\right), c_{b}=\left(s_{b}, \varphi_{b}\right)$ be two distinct conditional states of an FFSM $F F$ and also let $\rho \in \Lambda$ be a product configuration for $F F$ under a given SPL. If (i) $\rho \vDash \varphi_{a} \wedge \varphi_{b}$ (ii) there exists two valid conditional paths $\left(\left(c_{a}, \ldots, c_{a^{\prime}}\right), \delta, \gamma_{a}, \omega_{a}\right) \in \Theta_{F F}$ and $\left(\left(c_{b}, \ldots, c_{b^{\prime}}\right), \delta, \gamma_{b}, \omega_{b}\right) \in \Theta_{F F}$ such that $\rho \vDash \omega_{a} \wedge \omega_{b}$ and $\gamma_{a} \neq \gamma_{b}$, then a conditional test $\left(\delta,\left(\omega_{a} \wedge \omega_{b}\right)\right) \in I^{*} \times B(F)$ is called a separating sequence for $c_{a}$ and $c_{b}$ under the product $\rho$.

Note that for a given FFSM, there might be a single separating sequence that can distinguish a pair of states for every satisfiable product configuration. However, we need as many separating sequences as the number of valid product configurations.

Example 15. Given the FFSM FF presented in Figure 22 to distinguish the pair of conditional states $(1$, true $)$ and $(5$, true $)$ we use four transitions:

$$
\begin{aligned}
& (1, \text { true }) \stackrel{(c,(\neg W \vee S))}{\rightarrow 0}(1, \text { true }) \\
& (5, \text { true }) \stackrel{(c, \text { true })}{\rightarrow}(1, \text { true }) \\
& (1, \text { true }) \stackrel{(b, W)}{\rightarrow}(5, \text { true }) \\
& (5, \text { true }) \stackrel{(b, \text { true })}{\rightarrow}(5, \text { true })
\end{aligned}
$$

First, the set of product configurations that we need to cover using separating sequences is true which is equivalent to all product configurations of $\Lambda$. Then, from the transitions we obtain two separating sequences $(c,(\neg W \vee S))$ that covers all valid configurations except $\rho_{5}$, and $(b, W)$ to cover the missing configuration $\rho_{5}$. There are 6 valid configurations, but in this case we only need 2 separating sequences.

Given an FFSM and an SPL, there may be exponentially many number of valid product configurations, hence it may not be practical to derive a separating sequence for each valid product configuration. Instead, we compute separating sequences for sets of product configurations and put in a parametrized separating set.

Definition 4.4.7. Let $c_{a}=\left(s_{a}, \varphi_{a}\right), c_{b}=\left(s_{b}, \varphi_{b}\right)$ be two conditional states of an FFSM $F F$ such that there exists at least one product configuration $\rho \in \Lambda$ that satisfying both feature constraints, i.e., $\rho \vDash \varphi_{a} \wedge \varphi_{b}$. The set $P S_{a b} \in \mathcal{P}\left(I^{*} \times B(F)\right)$ is called a parametrized separating set for $c_{a}$ and $c_{b}$ if the disjunction of conditions of every separating sequence $(\delta, \omega) \in P S_{a b}$ (Definition 4.4.6) is equivalent to $\varphi_{a} \wedge \varphi_{b}$. Thus, $P S_{a b}$ can distinguish $c_{a}$ and $c_{b}$.

Example 16. From example 15 we know that $c_{a}=(1$, true $), c_{b}=(5$, true $)$ which results in $P S_{a b}=\{(c,(\neg W \vee S)),(b, W)\}$. 


\section{State Identification}

The configurable characterizing set $C W$ for state identification of FFSMs is built using parametrized separating sets for every conditional state pair of the FFSM.

Definition 4.4.8. Given an FFSM $F F=\left(F M, C, c_{0}, Y, O, \Gamma\right)$, with conditional state set $C=\left\{c_{1}, \ldots, c_{n}\right\}$, the set $C W \in \mathcal{P}\left(I^{*} \times B(F)\right)$ is a configurable characterizing set, if and only if, for all $1 \leq i, j \leq n$ with $i \neq j$ and $\Lambda_{i} \cap \Lambda_{j} \neq \varnothing$, there exists a parametrized separating set $P S_{i j} \subseteq C W$ (Definition 4.4.7).

Example 17. In example 16 we obtained the parametrized separating set for conditional states $c_{1}=(1$, true $)$ and $c_{5}=(5$, true $)$ of Figure 22. Combining other pairs of conditional states $c_{2}=(2, N), c_{3}=(3, W), c_{4}=(4, B)$, and $c_{6}=(6, S)$ we have:

$$
\begin{aligned}
& P S_{15}=\{(c,(\neg W \vee S)),(b, W)\}, P S_{12}=\{(a, N)\}, P S_{13}=\{(a, W)\} \\
& P S_{14}=\{(a, B)\}, P S_{16}=\{(b,(\neg W \wedge S)),(b,(W \wedge S))\} \\
& P S_{52}=\{(a, N)\}, P S_{53}=\{(a, W)\}, P S_{54}=\{(a, B)\} \\
& P S_{56}=\{(c, S)\}, P S_{23}=\varnothing, P S_{24}=\varnothing, P S_{26}=\{(a,(N \wedge S))\} \\
& P S_{34}=\varnothing, P S_{36}=\{(a,(W \wedge S))\}, P S_{46}=\{(a,(B \wedge S))\}
\end{aligned}
$$

Note that $c_{i}$ and $c_{j}$ pairs which results $\varnothing$ we have that $\Lambda_{i} \cap \Lambda_{j}=\varnothing$, and we do not need to find separating sequences. The resulting configurable characterizing set (after removing conditional prefixes - Definition 4.4.2) is $\operatorname{cpref}(C W)=\{(a,($ true $)),(b,((\neg W \wedge$ $S) \vee W)),(c,(\neg W \vee S))\}$.

The conditional HSI sets for state identification of FFSMs are built using the configurable characterizing set $C W$.

Definition 4.4.9. Given an FFSM $F F=\left(F M, C, c_{0}, Y, O, \Gamma\right)$, with conditional state set $C=\left\{c_{1}, \ldots, c_{n}\right\}$, the sets $C H_{1}, \ldots, C H_{n} \in \mathcal{P}\left(I^{*} \times B(F)\right)$ are conditional harmonized state identifiers, if and only if for all $1 \leq i, j \leq n$ with $i \neq j$ and $\Lambda_{i} \cap \Lambda_{j} \neq \varnothing$, there exists a common parametrized separating subset $P S_{i j} \subseteq C W_{i} \cap C W_{j}$ (Definition 4.4.7) with conditional prefixes from $C W$ that distinguishes $c_{i}$ and $c_{j}$ using separating sequences.

Example 18. The conditional characterizing set $C W$ of Example 17 for the FFSM $F F$ presented in Figure 22 is: $C W=\{(a,($ true $)),(b,((\neg W \wedge S) \vee W)),(c,(\neg W \vee S))\}$. The conditional HSI sets for the FFSM $F F$ are:

$$
\begin{aligned}
& C H_{1}=\{(a,(\text { true })),(b,((\neg W \wedge S) \vee W)),(c,(\neg W \vee S))\}, \\
& C H_{2}=\{(a,(N))\}, C H_{3}=\{(a,(W))\}, C H_{4}=\{(a,(B))\}, \\
& C H_{5}=\{(a,(\text { true })),(b,(W)),(c,(\neg W \vee S))\}, \text { and } \\
& C H_{6}=\{(a,(S)),(b,(S)),(c,(S))\} .
\end{aligned}
$$




\section{Extended HSI Method}

Now we have sets $C T C$ for transition coverage and conditional HSI sets for state identification. Then, the final configurable test-suite $C T S$ is the concatenation of $C T C$ with every $\mathrm{CH}_{i}$.

Definition 4.4.10. Given an FFSM $F F=\left(F M, C, c_{0}, Y, O, \Gamma\right)$, the configurable transition cover set $C T C$, and the conditional harmonized state identifier $C H_{i}$ sets, the extended conditional HSI method defines a complete configurable test-suite CTS for FF by concatenating every tuple of $C T C$ with every $C H_{i}$ set for each $c_{i} \in C$ such that only conditional tests of $C T C$ that reach $c_{i}$ are concatenated and the conjunction of constraints is satisfied:

$$
\begin{aligned}
\forall_{c_{i} \in C} & \bullet \forall_{(\delta, \omega) \in C T C} \bullet \exists_{\left(\left(c_{0}, \ldots, c_{i}\right), \delta, \gamma, \omega\right) \in \Theta_{F F}} \bullet \forall_{\left(\beta, \omega^{\prime}\right) \in C H_{i}} \bullet \\
& \exists_{\rho \in \Lambda} \bullet \rho \vDash\left(\omega \wedge \omega^{\prime}\right) \Longrightarrow\left(\delta \beta,\left(\omega \wedge \omega^{\prime}\right)\right) \in C T S
\end{aligned}
$$

Example 19. The complete configurable test-suite is obtained by concatenating $C T C$ of Example 14 with the conditional HSI sets presented in Example 18. The following set is a complete configurable test-suite for $F F$.

$$
\begin{aligned}
& \text { cpref }(C T S)=(\{(b a,(\neg W)),(b b,(\text { true })),(b c,(\text { true })),(c a,(\text { true })),(c b,(\text { true })), \\
& (c c,(\neg W \vee S)),(d a,(S)),(d b,(S)),(d c,(S)),(a a a,(\text { true })), \\
& (a c a,(N \vee B \vee(W \wedge \neg S))),(a c b,(N \vee B \vee(W \wedge \neg S))),(a c c,(N \vee B)), \\
& (b a a,(W)),(b b a,(W)),(b b b,(W)),(b c a,(W)),(b c b,(W)),(b c c,(W \wedge S)), \\
& (a b a a,(\text { true })),(a b b a,(\text { true })),(a b b b,(W)),(a b b c,(\neg W \vee S)), \\
& (a b c a,(t r u e)),(a b c b,(t r u e)),(a b c c,(\neg W \vee S)),(a c b a,(W \wedge S)), \\
& (a c b b,(W \wedge S)),(a c b c,(W \wedge S)),(a c c a,(W \wedge S)),(a c c b,(W \wedge S)), \\
& (a c c c,(W \wedge S)),(a d a a,(S)),(a d b a,(S)),(a d b b,(S)),(a d b c,(S)), \\
& (a d b d,(S)),(a d c a,(S)),(a d c b,(S)),(a d c c,(S)),(a d c d,(S)),(a d d b,(S)), \\
& (a d d c,(S)),(a d d d,(S)),(a d d a a,((N \vee B) \wedge S)),(a d d b b,((N \vee B) \wedge S)), \\
& (a d d b c,((N \vee B) \wedge S \wedge \neg W)),(a d d b d,((N \vee B) \wedge S \wedge \neg W)), \\
& (a d d c b,((N \vee B) \wedge S)),(a d d c c,((N \vee B) \wedge S)),(a d d c d,((N \vee B) \wedge S)), \\
& (a d d d b,((N \vee B) \wedge S)),(a d d d c,((N \vee B) \wedge S)),(a d d d d,((N \vee B) \wedge S))\})
\end{aligned}
$$

Moreover, the derivation operator results in $\Delta_{\rho_{3}}(C T S)=$ $\operatorname{pre} f(\{b a, b b, b c, c a, c b, a a a, a c a, a c b, a c c, a b a a, a b b a, a b b c, a b c a, a b c b, a b c c\}) \quad$ that $\quad$ is $\quad$ a complete test-suite for the FSM $M$ presented in Figure 7. 
Next, we state and prove that a complete configurable test-suite is a complete test-suite for all its valid product FSMs.

Theorem 9. If the set $C T S \subseteq \mathcal{P}\left(I^{*} \times B(F)\right)$ is an $n$-complete configurable test-suite for an FFSM $F F$, then $C T S$ contains an $n$-complete test-suite $T S \subseteq \mathcal{P}\left(I^{*}\right)$ for all derived product FSMs $\Delta_{\rho}(F F)$.

Proof. We prove the implication by contradiction. Let the set $T S \subseteq \mathcal{P}\left(I^{*} \times B(F)\right)$ be an $n$-complete configurable test-suite for an FFSM $F F=\left(F, \Lambda, C, c_{0}, Y, O, \Gamma\right)$ and the set $\Delta_{\rho}(T S) \subseteq \mathcal{P}\left(I^{*}\right)$ be not an $n$-complete configurable test-suite derived from a product configuration $\rho$ under a derived FSM $\Delta_{\rho}(F F)=\left(S, s_{0}, I, O, T\right)$. As $\Delta_{\rho}(T S)$ is not an $n$ complete configurable test-suite for state $\Delta_{\rho}(F F)$, then does not exist a test $\delta h \in \Delta_{\rho}(T S)$ such $\delta$ exists in a path to reach a state $s$ and $h$ distinguishes $s$ with another state $s^{\prime}$. By Definition 4.4.9 and 4.4.10 for each conditional state $c_{i} \in C$ we have at least one conditional test $\left(\delta h,\left(\omega \wedge \omega^{\prime}\right)\right) \in T S, \rho \vDash\left(\omega \wedge \omega^{\prime}\right)$ where $(\delta, \omega) \in C T C$ reaches $c_{i}$ and $\left(h, \omega^{\prime}\right) \in C H_{i}$ distinguishes $c_{i}$ with another conditional state $c_{j} \in C, i \neq j$. By Definition 4.4.3 the conditional input sequence $\left(\delta h,\left(\omega \wedge \omega^{\prime}\right)\right) \in T S$ derives an input sequence $\delta h \in \Delta_{\rho}(T S)$ for $\rho$, which is a contradiction as $\delta h \notin \Delta_{\rho}(T S)$.

\subsection{Concluding Remarks}

In this chapter, we presented the Featured Finite State Machine (FFSM) that is a behavioral test model for software product lines (SPLs). We extended the HSI test-case generation method from FSMs to FFSMs. The HSI test generation method was originally designed to generate tests using FSMs for the full fault coverage criteria. However, FSMs used as inputs to HSI require semantic properties such as determinism, initially connected and minimal. Thus, validation properties were specified for adopting FFSMs as input models for test-case generation algorithms and we showed that they coincide with their corresponding properties for the product FSM models. Finally, configurable test-suites were defined and explained. 



\section{HIERARCHICAL FEATURED STATE MACHINE}

Inspired by Harel's Statecharts (HAREL; NAAMAD, 1996), several hierarchical state machine formalisms were defined to specify the behavioral aspects of reactive systems and extended to object-oriented software development methodologies such as the Unified Modeling Language (UML) (OMG, 2015). The states represented in the hierarchical model can be simple states or contain an entire state machine. Systems can be specified by a stepwise refinement and visualized in different levels of granularity at the cost of complex syntax and semantics.

To design configurable models and tests one must first validate the model to check properties required for specific test criteria. In this thesis, we present the Hierarchical Featured State Machine (HFSM) formalism that extends the FFSM model including hierarchy. Due to scalability problems, e.g., the state explosion problem, large systems are hard to maintain using the FFSM model.

In this thesis, we present the Hierarchical Featured State Machine (HFSM) formalism that extends the FFSM model including hierarchy. The HFSM model improves model readability by grouping up FFSM conditional states and transitions into an abstracted view, which provides a better solution for modeling SPL-based test models in the ModelBased Testing (MBT) approach. Furthermore, the HFSM model can also be pruned for subsets of product configurations.

In our approach, we use the HFSM as a front end for modeling and syntax check while the semantics are represented by an FFSM. However, one of the main issues of lifting the FFSM formalism to HFSM is how to compose orthogonal regions.

The remainder of this chapter is organized as follows. Section 5.1 introduces detailed syntax of HFSMs, followed by its semantics in Section 5.2. Section 5.3 proposes similar 
validation properties to FFSMs, and finally, the test design approach proposed in this thesis in Section 5.4.

\subsection{Syntax}

Tere are many variations of hierarchical FSMs that we can use to reuse to define an HFSM. We choose a definition in which there are no final states and inspired terminology from UML (OMG, 2015). Thus, an HFSM comprises states that might have a further internal structure (hierarchy) and transitions among them. States and transitions have feature constraints that must be satisfied according to a feature model. The following definitions are based on the corresponding definitions in (HAREL; NAAMAD, 1996).

Definition 5.1.1. A Hierarchical Featured State Machine HF is represented by a 5-tuple $(F M, \Upsilon, I, O, \mathcal{T})$, where:

1. $F M=(F, \chi, \Lambda)$ is a feature model (Definition 2.3.1),

2. $\Upsilon$ is a well-formed state structure,

3. $I$ is a set of inputs events,

4. $O$ is a set of outputs events,

5. $\mathcal{T}$ is a set of well-formed transitions.

A well-formed state structure is a tree based on (HAREL; NAAMAD, 1996) that represent a valid state hierarchy. The set of well-formed transitions contains valid transitions that connect sets of states in the hierarchy. Input and output sets represent the observable behavior of the machine.

Example 20. Figure 25 shows the HFSM for the AGM SPL (SEI, 2011). Similar to the notation for FFSM presented in Example 9 we alternatively do not compose the alternative states and represent them in regions. Note that we have only one state per alternative feature and further in the semantics section we present examples where this is not the case and a combination of states are required. Internal behavior represents self-loop transitions and inputs with equivalent feature constraints for the same output can be grouped by ",". For simplicity, we do not treat history, deep-history, join/forks, and entry/exit points connections in this thesis.

Not every possible state structure is valid. Transitions also have to be checked, since a transition must not target mutually exclusive states. Thus, some criteria are required to represent well-formed state structures and transitions. 


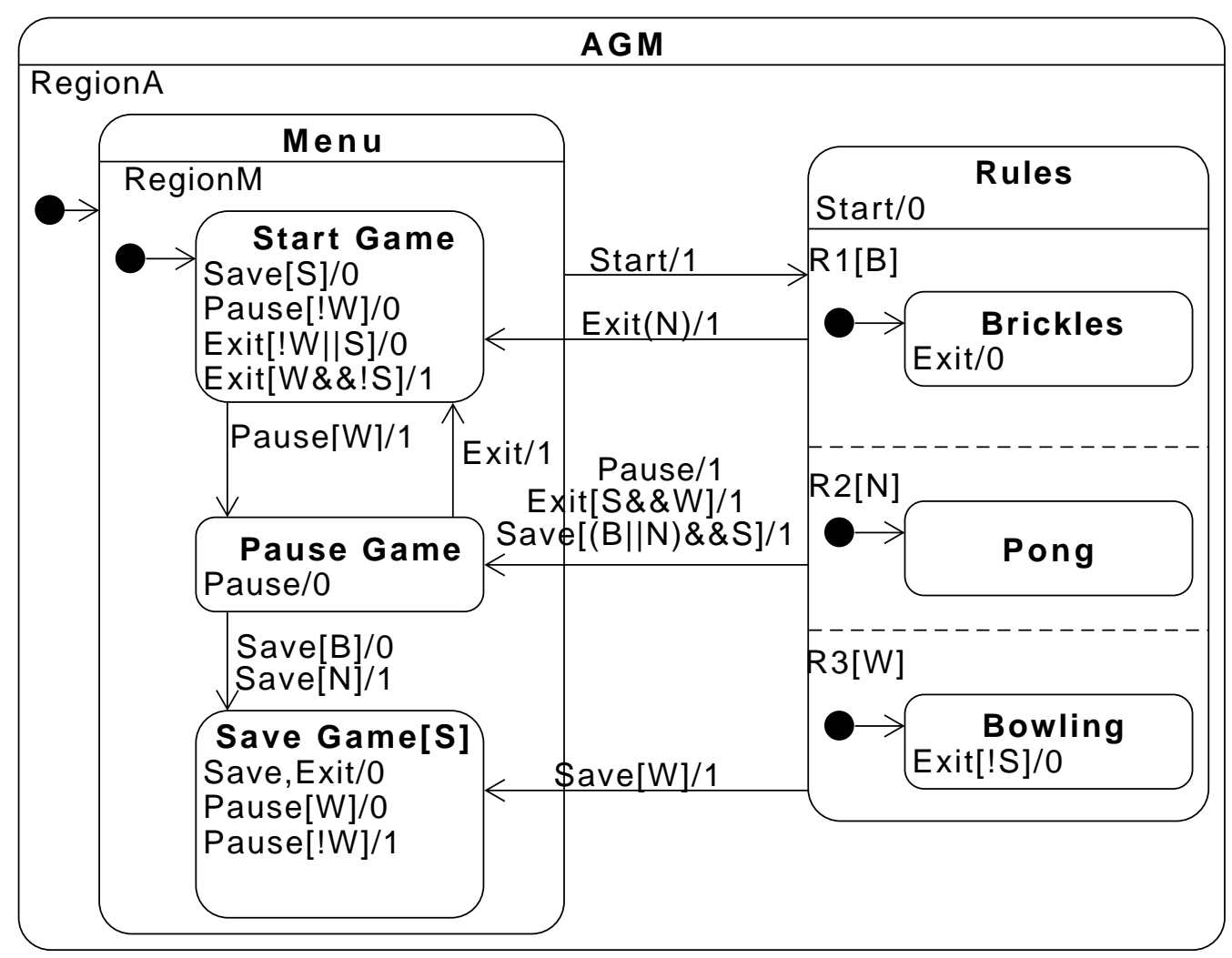

Figure 25 - HFSM for AGM.

\subsubsection{Well-formed state structure}

We define the syntactic state structure of HFSMs, their restrictions, and wellformedness conditions. First, we define the state structure as follows.

Definition 5.1.2. A state structure $\Upsilon$ is defined by a 6-tuple ( $S$, root, de fault, sub, type, feature), where:

1. $S$ is a finite set of states;

2. root $\in S$ is the root state of the state structure;

3. default: $S \rightarrow\{$ true, false $\}$, is a total function determining whether a state is default or not;

4. $s u b: S \rightarrow \mathcal{P}(S)$ is a total function defining for each state, the set of its sub-states;

5. type $: S \rightarrow\{$ simple, compOr, compAnd,region $\}$ is a total function determining for each state whether it is a simple (state with no sub-states), a compOr (a composite state with only one default sub-state), a compAnd (a composite state with more than one default sub-state), or a region state (sub-states of a composite state);

6. feature: $S \rightarrow B(F)$, is a total function determining the feature constraint of a state. 


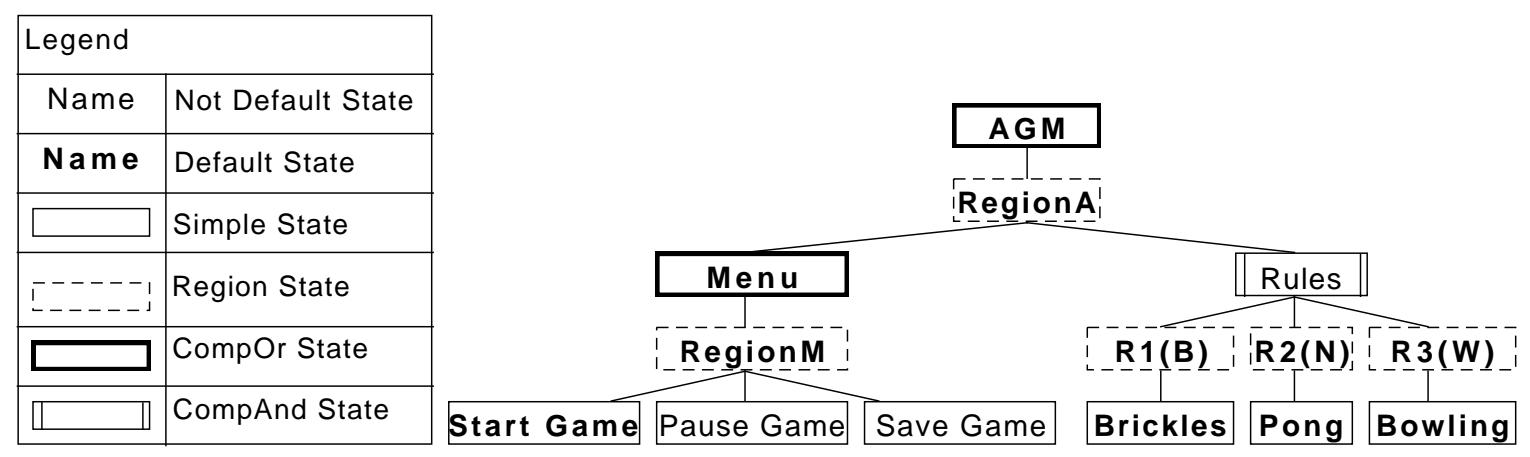

Figure 26 - State structure for AGM HFSM.

A tree of conditional states can represent the state structure. The root state is the unique state that encapsulates all states in the state structure. A default state is a state that is automatically activated once the super-state (father) is activated or it is the root itself. In Figure 25, the default states, besides region states, are represented by default connectors. The sub-states of a state are their children in the tree structure.

Example 21. Figure 26 shows the state structure of the HFSM presented in Figure 25, where $A G M$ is the root state, Rules is the CompAnd state, most of the state are default states, and sub-states of the RegionA state is $\operatorname{sub}($ Region $A)=\{$ Menu, Rules $\}$.

The state structure is generic and allows inconsistent specifications, e.g., sub-states of a region state being region states. To validate the state structure we need to define several auxiliary functions, and finally, formalize the well-formed state structure. First, we define hierarchy using descendants and ancestors. Descendants refer to sub-states of sub-states and so forth.

Definition 5.1.3. Given a state $s \in S$, the set of descendants of $s$ is denoted by desc $(s)$ and satisfies the following conditions.

1. $\{s \cup \operatorname{sub}(s)\} \subseteq \operatorname{desc}(s)$; and

2. $\forall_{s^{\prime}, s^{\prime \prime} \in S} \bullet s^{\prime} \in \operatorname{sub}(s) \wedge s^{\prime \prime} \in \operatorname{desc}\left(s^{\prime}\right) \Longrightarrow s^{\prime \prime} \in \operatorname{desc}(s)$.

For a set of states $S^{\prime} \subseteq S$, we define the notion of descendant by $\operatorname{Desc}\left(S^{\prime}\right)=$ $\bigcup_{s^{\prime} \in S^{\prime}} \operatorname{desc}\left(s^{\prime}\right)$. Super-states and ancestors are the opposite of sub-states and descendants, respectively, and defined as follows.

Definition 5.1.4. Given a state $s \in S$, a super-state (father) of $s$ is defined by a function super $: S \rightarrow S$ where $\exists_{s^{\prime} \in S} \bullet s \in \operatorname{sub}\left(s^{\prime}\right) \Longleftrightarrow s^{\prime}=\operatorname{super}(s)$. Moreover, ancestors of $s$ are denoted by anc $(s)$ and satisfy the following condition. 


$$
\forall_{s, s^{\prime} \in S} \bullet s \in \operatorname{anc}\left(s^{\prime}\right) \Longleftrightarrow s^{\prime} \in \operatorname{desc}(s)
$$

For a set of states $S^{\prime} \subseteq S$, we define the notion of ancestor by $\operatorname{Anc}\left(S^{\prime}\right)=\bigcap_{s^{\prime} \in S^{\prime}} \operatorname{anc}\left(s^{\prime}\right)$.

Example 22. Following Figure 26 some descendants and ancestors in the state structure of the HFSM are:

- $\operatorname{desc}(M e n u)=\{$ Menu, RegionM, StartGame,PauseGame, SaveGame $\}$

- $\operatorname{anc}($ PauseGame $)=\{$ PauseGame, RegionM, Menu, RegionA, AGM $\}$;

- $\operatorname{Desc}(\{$ Region $M, R 1\})=\{$ Region $M, R 1$, StartGame, PauseGame, SaveGame, Brickles\}; and

- Anc $(\{$ PauseGame, Brickles $\})=\{$ Region $A, A G M\}$.

In the FFSM representation, a conditional state generalizes the feature constraint on the transitions. Similarly, in the HFSM representation, the feature constraint of a state generalizes the constraint of its descendants. Thus, we define a composition of constraints that is used to check states in the hierarchy.

Definition 5.1.5. Given a set of states $S$, the state feature composition, denoted by fcomp : $\mathcal{P}(S) \rightarrow B(F)$ is the conjunction of feature constraints of $S$ :

$$
f \operatorname{comp}(S)=\bigwedge_{s \in S} \text { feature }(s)
$$

Finally, we define the concept of well-formedness of state structures extended with feature constraints from the corresponding restrictions in (HAREL; NAAMAD, 1996).

Definition 5.1.6. The state structure $\Upsilon=(S$, root, default, sub, type, feature $)$ is wellformed, when:

1. Simple-states have no sub-states.

$$
\forall_{s \in S} \bullet \operatorname{type}(s)=\text { simple } \Longrightarrow \operatorname{sub}(s)=\varnothing
$$

This is a basic assumption as simple states should not have any further structure inside them.

2. All nodes, besides root, have a unique super-state.

$$
\forall \forall_{s \in S \backslash\{\text { root }\}} \bullet \exists !_{s^{\prime} \in S} \bullet s^{\prime}=\operatorname{super}(s)
$$


Make sure that the chain of ancestors reaches an end in the root. Also, make sure that every internal behavior is encapsulated by regions states.

3. Strict descendance relation is asymmetric.

$$
\forall_{s, s^{\prime} \in S} \bullet s \in \operatorname{desc}\left(s^{\prime}\right) \backslash\left\{s^{\prime}\right\} \Longrightarrow s^{\prime} \notin \operatorname{desc}(s) \backslash\{s\}
$$

The constraint disallows loops in the chain of ancestors and descendants.

4. The single sub-state of a compOr state is a region state.

$$
\forall_{s \in S} \bullet \operatorname{type}(s)=\operatorname{compOr} \Longrightarrow \exists !_{s^{\prime} \in S} \bullet s^{\prime} \in \operatorname{sub}(s) \wedge \operatorname{type}\left(s^{\prime}\right)=\text { region }
$$

Upon entering a compOr state, we enter in the single region sub-state that represent an inner machine.

5. All sub-states of compAnd states are region states.

$$
\begin{array}{r}
\forall_{s \in S} \bullet \operatorname{type}(s)=\text { compAnd } \Longrightarrow \forall_{s^{\prime}, s^{\prime \prime} \in \operatorname{sub}(s)} \bullet \operatorname{type}\left(s^{\prime}\right)=\text { region } \wedge \\
\operatorname{type}\left(s^{\prime \prime}\right)=\text { region }
\end{array}
$$

By definition a compAnd state have more than one region sub-state. Upon entering an compAnd state, we enter in all region sub-states to support parallelism.

6. Region states are default states, their sub-states must not be region states, and only one of their sub-states is default.

$$
\begin{array}{r}
\forall_{s \in S} \bullet \operatorname{type}(s)=\text { region } \Longrightarrow \operatorname{default}(s) \wedge \forall_{s^{\prime} \in \operatorname{sub}(s)} \bullet \operatorname{type}\left(s^{\prime}\right) \neq \operatorname{region} \wedge \\
\exists ! s_{s^{\prime \prime} \in \operatorname{sub}(s)} \bullet \operatorname{default}\left(s^{\prime \prime}\right)
\end{array}
$$

Upon entering a composite state, we automatically enter in a region state that contains an inner machine. Region states are special states used to represent inner machines, and only composite states can have region states as sub-states. One sub-state of the region state must be default to represent the "initial state" of this region. As consequence, every non-region state, besides root, has an unique region super-state.

7. Root is of type compOr and all states are descendants of the root.

$$
\exists !_{s \in S} \bullet s=\operatorname{root} \wedge \operatorname{type}(s)=\operatorname{compOr} \wedge \forall_{s^{\prime} \in S} \bullet s^{\prime} \in \operatorname{desc}(s)
$$


The root state is the common ancestor state of all states in the state structure.

8. The feature constraint of every state is satisfied by at least one product (Definition $5.1 .5)$.

$$
\forall_{s \in S} \bullet \exists_{\rho \in \Lambda} \bullet \rho \vDash f \operatorname{comp}(\operatorname{anc}(s))
$$

When a state is active it means that every ancestor is active as well. Thus, the hierarchy must not contain branches that are not satisfied by any product configuration.

\subsubsection{Well-formed transitions}

A transition connects states using input events that trigger output events when its conditions are satisfied. Next, we formalize the definition of transitions and the wellformedness criteria.

Definition 5.1.7. A transition $t$ in an $\operatorname{HFSM}$ is defined by a 5 -tuple $(A, i, \omega, o, B)$, where:

1. $A \subseteq S$ is the set of source states;

2. $i \in I$ is the input event;

3. $\omega \in B(F)$ is the feature constraint of the transition;

4. $o \in O$ is the output event;

5. $B \subseteq S$ is the set of target states.

A transition $t=(A, i, \omega, o, B)$ is denoted by $A \underset{o}{\stackrel{(i, \omega)}{\rightarrow}} B$. The source states are those where the transition begins, while the target states are those where it ends. In general, most transitions connect a pair of states and only use several states as a source or target states when there are parallel/orthogonal states. The input and output events are the observable behavior of the transition. The feature constraint $\omega$ is a specific condition of the transition. When we omit the feature constraint, $\omega=$ true. States of source and target sets generalize the entire feature constraint of a transition. Thus, we compose the feature constraints of all elements of the transition that are used for checking, that is defined as follows.

Definition 5.1.8. Given a transition $t=(A, i, \omega, o, B)$, the transition feature composition, denoted by tcomp : $\mathcal{T} \rightarrow B(F)$ is the conjunction of feature constraints of elements of $t$ :

$$
\operatorname{tcomp}(t)=\left(\bigwedge_{a \in A} f \operatorname{comp}(\operatorname{anc}(a))\right) \wedge \omega \wedge\left(\bigwedge_{b \in B} f \operatorname{comp}(\operatorname{anc}(b))\right)
$$

A transition exists in a product configuration only if it can satisfy the transition feature composition. The definition of transition is generic and allows for inconsistent 
specifications, e.g., transitions that the target state is a region state. Transitions are not supposed to connect to arbitrary types of states. To validate a transition we need to define several auxiliary functions, and finally define the well-formed transition. Thus, we define the subset of states that can be used as source and target in a transition.

Definition 5.1.9. Given the set of states $S$, the set of transition relevant states, denoted by $R \subset S$ is the subset of states that are not region states nor the root state:

$$
\forall_{s \in S} \bullet \operatorname{type}(s) \neq \text { region } \wedge s \neq \text { root } \Longrightarrow s \in R
$$

Next, we define the notion of the least common ancestor. To define orthogonality between two states we check their least common ancestor.

Definition 5.1.10. Given a set of states $S$, their least common ancestor, denoted by $l c a(S)$, is the bottommost ancestor which contains all states.

$$
s=l c a(S) \Longleftrightarrow s \in A n c(S) \wedge \forall_{s^{\prime} \in \operatorname{Anc}(S)} \bullet s^{\prime} \in \operatorname{anc}(s)
$$

Example 23. The relevant states for a transition and the least common ancestor of PauseGame and Brickles states are:

- $R=\{$ Menu, Rules, StartGame, PauseGame,Brickles, Pong,Bowling $\}$;

- lca $(\{$ PauseGame, Brickles $\})=$ RegionA.

Definition 5.1.11. Two states $s$ and $s^{\prime}$ are orthogonal to each other when their least common ancestor is a compAnd state. If $s$ and $s^{\prime}$ are not orthogonal, then the $l c a\left(s, s^{\prime}\right)$ is: $s$ when $s^{\prime} \in \operatorname{desc}(s)$; $s^{\prime}$ when $s \in \operatorname{desc}\left(s^{\prime}\right)$; or super $(s)$ when $\operatorname{super}(s)=\operatorname{super}\left(s^{\prime}\right)$ that is a region state. A set of states $S^{\prime} \subseteq S$ is orthogonal if all its states are pairwise orthogonal.

$$
\forall_{s, s^{\prime} \in S^{\prime}} \bullet \operatorname{type}\left(l c a\left(\left\{s, s^{\prime}\right\}\right)\right)=\operatorname{compAnd}
$$

Only orthogonal states can be active at one time. Transitions that are not fired in one region may be fired in another parallel region state.

Finally, we define the concept of well-formedness of transitions extended with feature constraints from the corresponding restrictions in (HAREL; NAAMAD, 1996).

Definition 5.1.12. A transition $t=(A, i, \omega, o, B)$ is well-formed when the following conditions hold.

1. The source and target states are transition relevant states. 


$$
\forall_{a \in A, b \in B} \bullet a, b \in R
$$

This is a basic assumption as no transition should use regions or root.

2. The set of source states and target states are pairwise orthogonal.

$$
\begin{aligned}
& \forall_{a, a^{\prime} \in A} \bullet \operatorname{type}\left(\operatorname{lca}\left(\left\{a, a^{\prime}\right\}\right)\right)=\mathrm{compAnd} \wedge \\
& \quad \forall_{b, b^{\prime} \in B} \bullet \operatorname{type}\left(\operatorname{lca}\left(\left\{b, b^{\prime}\right\}\right)\right)=\mathrm{comp} \text { And }
\end{aligned}
$$

Transitions can only connect set of states that are orthogonal to each other.

3. The source and target states are not orthogonal to each other.

$$
\forall_{a \in A, b \in B} \bullet \operatorname{type}(l c a(\{a, b\})) \neq \operatorname{compAnd}
$$

There should not be any transition involving parallel regions. Communication mechanisms are used to trigger transitions in parallel regions.

4. The transition $t$ has to be satisfied by at least one product (Definition 5.1.8).

$$
\exists_{\rho \in \Lambda} \bullet \rho \vDash t \operatorname{comp}(t)
$$

Every transition exists in at least one product configuration.

\subsection{Semantics}

The semantics of HFSMs are represented using FFSMs. FFSM conditional states are based on HFSM configurations.

\subsubsection{Mapping HFSM to FFSM}

A configuration is a set of active states that the system can be in simultaneously. The configuration always has the root and at least one simple state (leaf of the state structure). Initially, the root state is automatically activated. Then, all default descendants are activated as well.

Definition 5.2.1. Given a state $s \in S$, the set of default descendants is denoted by $\operatorname{ddesc}(s)$ and satisfies the following condition. For every state $s^{\prime} \in S$ if $s^{\prime}$ is a descendant of $s$ and every ancestor of $s^{\prime}$ excluding the ancestors of $s$ are default, then $s^{\prime} \in \operatorname{ddesc}(s)$ : 


$$
\exists_{s, s^{\prime} \in S} \bullet \forall_{s^{\prime \prime} \in \operatorname{anc}\left(s^{\prime}\right) \backslash \operatorname{anc}(s)} \bullet s^{\prime} \in \operatorname{desc}(s) \wedge \operatorname{default}\left(s^{\prime \prime}\right) \Longleftrightarrow s^{\prime} \in \operatorname{ddesc}(s)
$$

For a set of states $S^{\prime} \subseteq S$, we define the notion of default descendant by $\operatorname{DDesc}\left(S^{\prime}\right)=$ $\bigcup_{s^{\prime} \in S^{\prime}} \operatorname{ddesc}\left(s^{\prime}\right)$.

The default descendants of root represent the initial configuration of the HFSM. The state set Init is used to denote the initial configuration, i.e., $\forall_{s \in S} \bullet s \in \operatorname{ddesc}($ root $) \Longrightarrow$ $s \in$ Init.

Transitions of the HFSM connect configurations in a limited scope area of the state structure.

Definition 5.2.2. Given a transition $t=(A, i, \omega, o, B)$, the scope of $t$ is denoted by scope $(t)$ and is the lowest state in the state hierarchy that is a proper common ancestor of all source and target states, i.e., scope $(t)=l c a(\{A \cup B\})$.

Example 24. The initial configuration of the state structure presented in Figure 26 is Init $=\{$ root,Region A, Menu, RegionM,StartGame $\}$, and given a transition $t=(\{$ Menu $\}, i, \omega, o,\{$ Brickles $\})$, the scope of $t$ is scope $(t)=$ Region $A$.

When a transition is performed some states are deactivated while some are activated. Thus, states which are exited or entered as a result of taking a transition, are not necessarily identical to sources or targets of that transition. For source states, all its active descendants are exited and sometimes some of its ancestors. For target states, some of its ancestors are entered and all of its default descendants are entered and activated.

The resulting conditional state set of the semantic FFSM is all simple states that do not have a CompAnd state as one of its ancestors plus all composed states (see subsection $5.2 .2)$.

For transitions the semantic FFSM contains transitions following some cases:

- If a transition $t$ reaches a CompAnd state $s$ then the target state the initial state of the composition of $s$.

- If a transition $t$ reaches a CompOr state $s$ then the target state is the unique simple state of the default descendants of $s$.

- If a transition $t$ reaches a simple state $s$ then compose $s$ with a set of orthogonal states (if it is the case). Thus, select all ancestors of $s$ except the ancestors of the scope of $t$, then if any of those selected states are a compAnd state select all default descendants of it except the single state that is a sub-state of one of the ancestors of $s$. 
- If a transition $t$ leaves a CompAnd state $s$ then every resultant composed state of $s$ is a source state for a new transition.

- If a transition $t$ leaves a CompOr state $s$ then every descendant of $s$ that is a simple state is a source state for a new transition.

- If a transition $t$ leaves a simple state $s$ then compose $s$ with a set of orthogonal states (if it is the case). Thus, select all ancestors of $s$ except the ancestors of the scope of $t$, then if any of those selected states are a compAnd state compose all region combinations except the region that is an ancestor of $s$.

Example 25. The FFSM conditional states are

$\{$ StartGame, PauseGame, $($ SaveGame,$(S)),($ Brickles $*$ Pong $*$ Bowling,$(B \vee N \vee$ $W))\}$

Assume two transitions of AGM HFSM from Figure 25:

$t_{1}=(\{$ Menu $\}, i, \omega, o,\{$ Rules $\})$,

$t_{2}=(\{$ Rules $\}, i, \omega, o,\{$ PauseGame $\})$.

The resultant transitions of the FFSM for $t_{1}$ are:

$t_{11}=(\{$ StartGame $\}, i, \omega, o,\{($ Brickles $*$ Pong $*$ Bowling,$(B \vee N \vee W))\})$,

$t_{12}=(\{$ PauseGame $\}, i, \omega, o,\{($ Brickles $*$ Pong $*$ Bowling,$(B \vee N \vee W))\})$,

$t_{13}=(\{($ SaveGame,$(S))\}, i, \omega, o,\{($ Brickles $*$ Pong $*$ Bowling,$(B \vee N \vee W))\})$.

The resultant transition of the FFSM for $t_{2}$ is:

$t_{21}=(\{($ Brickles $*$ Pong $*$ Bowling,$(B \vee N \vee W))\}, i, \omega, o,\{$ PauseGame $\})$.

\subsubsection{Composition of Orthogonal States}

To compose states of CompAnd type we start with the innermost state and go back composing until it gets back to the root state. Figure 27 shows the simple composition of three regions where the configurations are identified in incremental paired steps. On (a) we have an example with two states each. On (b) we show the composition order where (c) shows the first composition step and (d) the second step. In this combination of regions, we have some unreachable configurations that are not considered to the semantics. However, without Region 2 the configurations would be different. For example, the composed output $o_{1} * o_{2}$ of the transition $\left(a_{1} * b_{1}\right) \underset{o_{1} * o_{2}}{\stackrel{a}{\rightarrow}}\left(a_{2} * b_{2}\right)$ would be only $o_{1}$ for transition $a_{1} \underset{o_{1}}{\stackrel{a}{\rightarrow}} a_{2}$.

For HFSMs we also have to consider the possibility of a region exist in every product configuration or not. Figure 28 shows three different situations where the feature model structure affects the combination of region compositions. In the normal scenario (a) where there is only one possible combination of regions, the composition is similar 


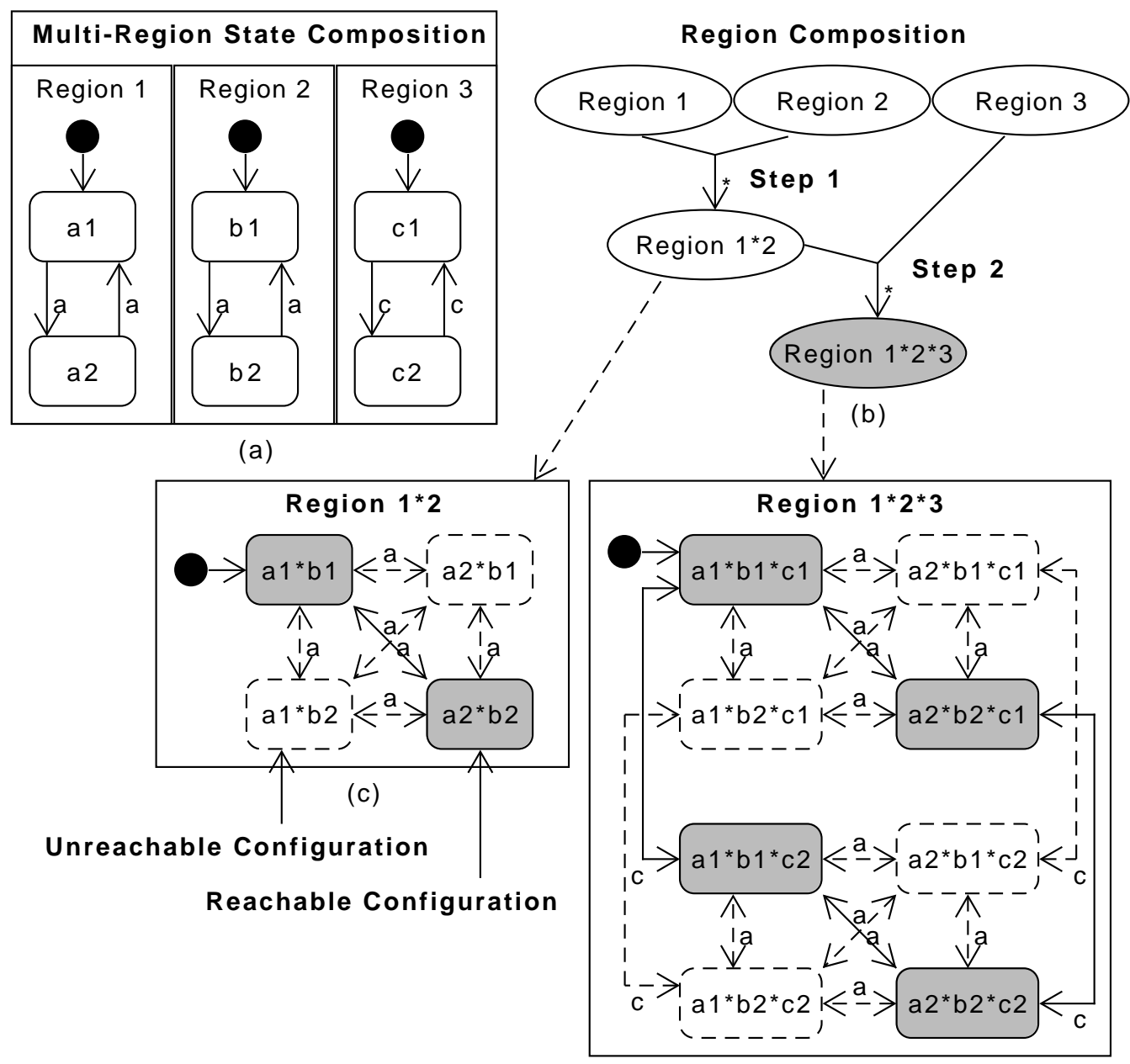

(d)

Figure 27 - Simple composition of regions states of a CompAnd state.

to Figure 27. In scenario (b) we have three alternative features mapped to each region which the composition is made on each single region and adding the combination feature constraint to each composed state. On (c) we have optional features which have seven possible combinations of region composition. For each combination, a region composition is performed, and each initial state is merged into the Init state, and its feature constraint is a disjunction of the region conditions.

\subsection{Validation and Test Generation}

An FSM can represent the behavior of a single SPL product. A valid HFSM provides an integrated vision of the behavior of all products in a single model. HFSMs are simplified test models for conformance testing of SPLs that add feature constraints to states and transitions using state hierarchy. 


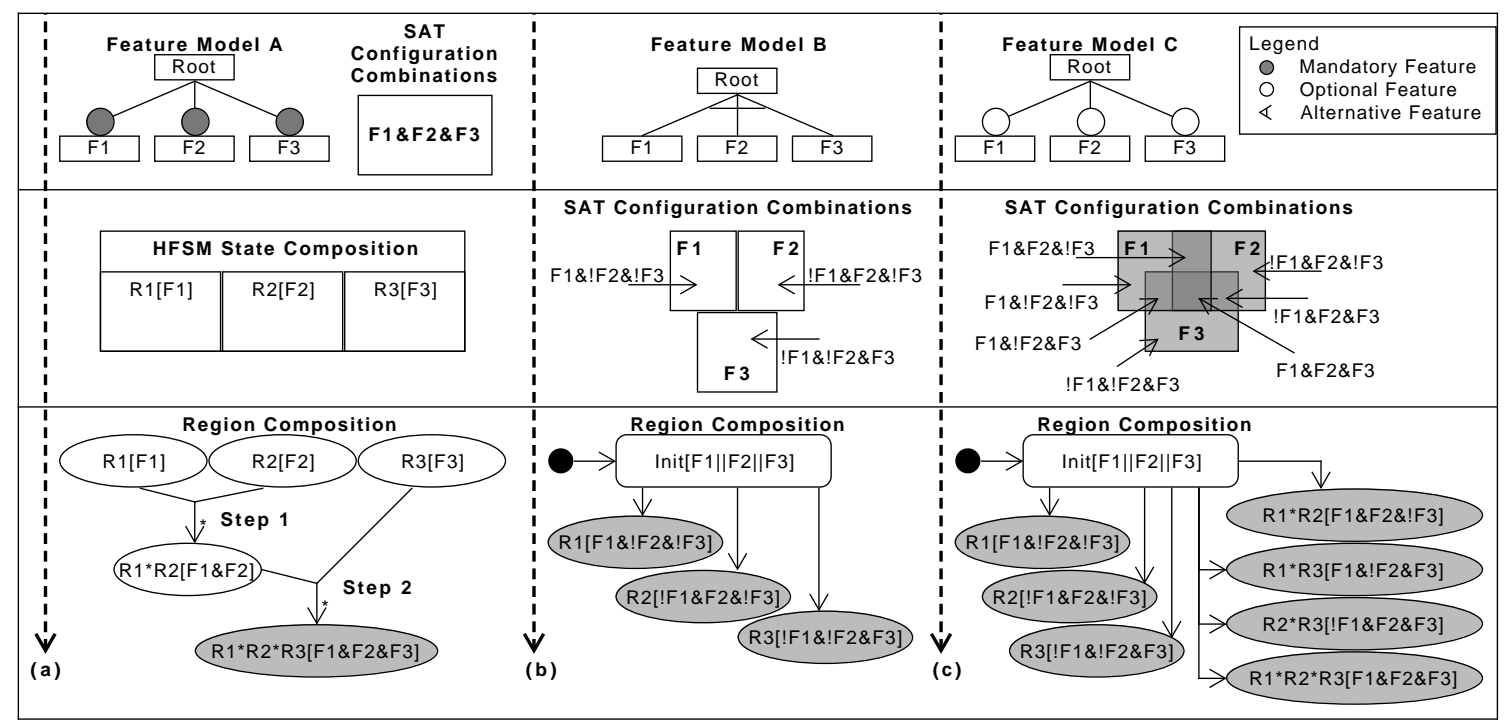

Figure 28 - HFSM composition of regions states of a CompAnd state.

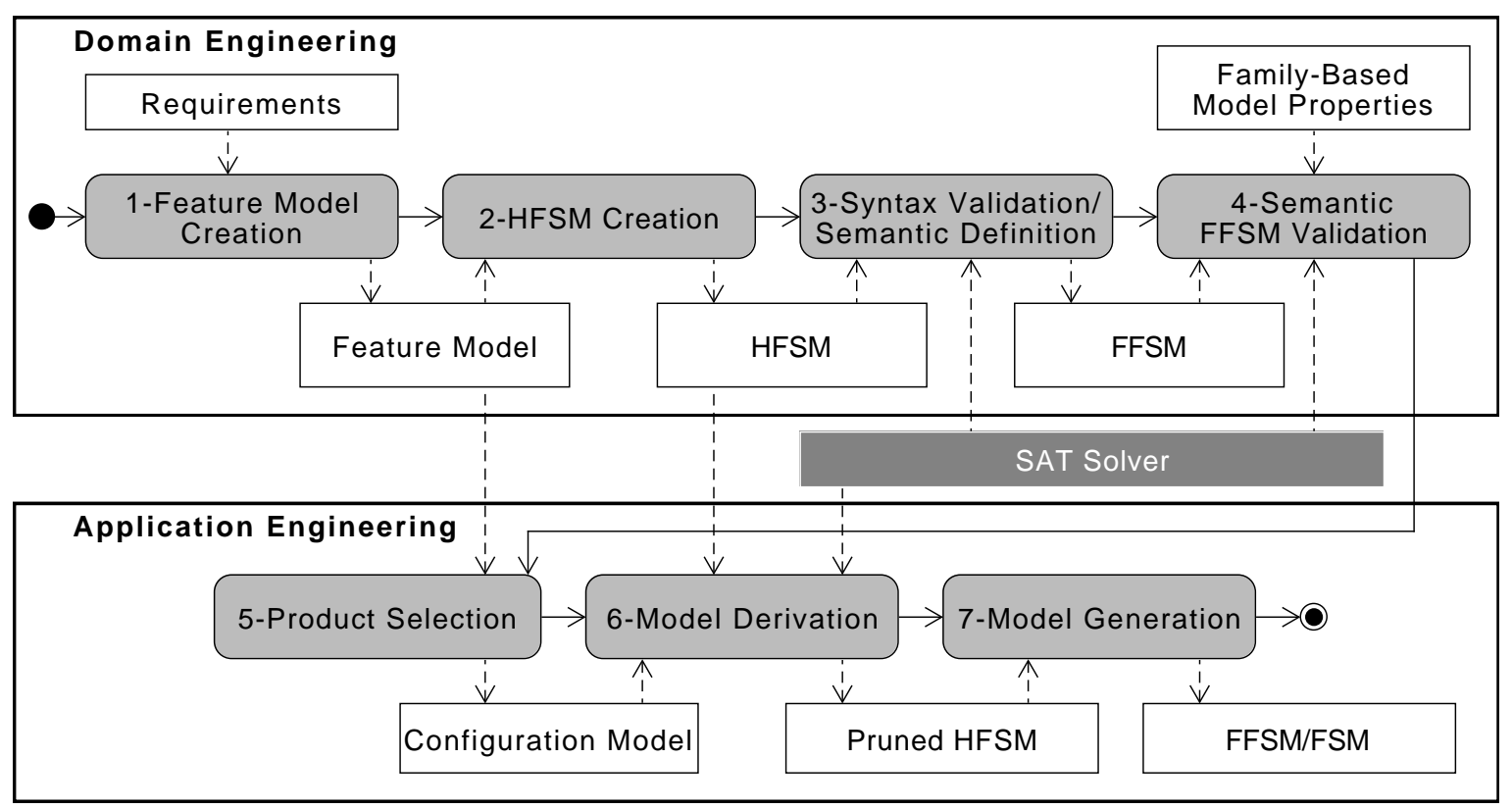

Figure 29 - SPL validation workflow using HFSMs.

Test-suites are generated using the semantic FFSM generated from the HFSM. However, we first need to validate the syntax of the HFSM. Figure 29 shows an overview of the SPL validation workflow for HFSM with artifact dependency represented by dashed arrows. On domain engineering, we generate reusable and configurable artifacts while in application engineering those artifacts can be configured for a set of product configurations.

In this thesis, we propose a solution named Configurable Feature-based full Coverage testing of State Machines (CFC-SM). CFC-SM is a model-based test design approach 
that enables the automatic generation of reusable and customizable test artifacts for an SPL, which can be configured to test a set of products. The test engineer uses CFC-SM to create configurable feature-based state machines and generates test-cases for the full fault coverage criteria, specifically extending the HSI method (LUO et al., 1994). The CFC-SM approach has seven steps:

1. Create a feature model of the SPL;

2. Create a single configurable feature-based state machine that represents the behavior of the SPL;

3. Validate the feature-based state machine to apply the full fault coverage criteria;

4. Generate configurable test-cases using a feature-based state machine;

5. Instantiate the feature model in a configuration model for a single or a set of products;

6. Prune the feature-based state machine using the configuration model;

7. Prune test-cases using a configuration model.

On Step 4 test-cases can be generated for the state, transition, and full fault coverage criteria.

\subsection{Concluding Remarks}

In this chapter, we described the Hierarchical Featured State Machine (HFSM) that is a behavioral test model for software product lines (SPLs). The HFSM is a test model design inspired by statecharts that include state hierarchy to extend the FFSM formalism. Some validation properties used for FFSMs were proposed for HFSMs as well. Finally, the Configurable Feature-based full Coverage testing of State Machines (CFC-SM) approach was introduced that is a solution to family-based test design for SPLs. 
Part III

Application 

CHAPTER

6

(1)

IMPLEMENTATION

Tool support is essential to manage test models and guide test design. Once the theory is implemented the community are more likely to check out what have been done to improve the research topic. Moreover, using tools, we can create testing frameworks for more elaborate experiments allowing better analysis.

The remainder of this chapter is organized as follows. Section 6.1 presents the implemented tool that was used to support this thesis, and then the importance of the SAT Solver to check feature constraints and how it is used with the Java language in Section 6.2.

\subsection{Configurable Full Test Generator Tool}

To support the techniques proposed in this thesis, we have developed a test design tool Configurable Full Test Generator (ConFTGen). The ConFTGen has a graphical editor based on the Eclipse platform that was extended from the Yakindu GitHub Project ${ }^{1}$ (Eclipse Public Licence).

The Eclipse Platform is a framework that provides an open source software development environment. Eclipse has a core architecture that supports the integration of tools and other development environments. Moreover, Eclipse projects are implemented in Java language and can be run on several operating systems including Linux, Mac OS $\mathrm{X}$, and Windows. The functional unit of Eclipse is plug-ins. Plug-ins are combined with the core architecture and can be integrated to build complex tools. The core plug-ins used to develop the ConFTGen tool were org.eclipse.core, org.eclipse.ui, org.eclipse.gef, org.eclipse.emf, org.eclipse.gmf.

ConFTGen is integrated with FeatureIDE (THÜM et al., 2014) (Lesser General

1 Open Source Yakindu Project <https://github.com/Yakindu/statecharts> 


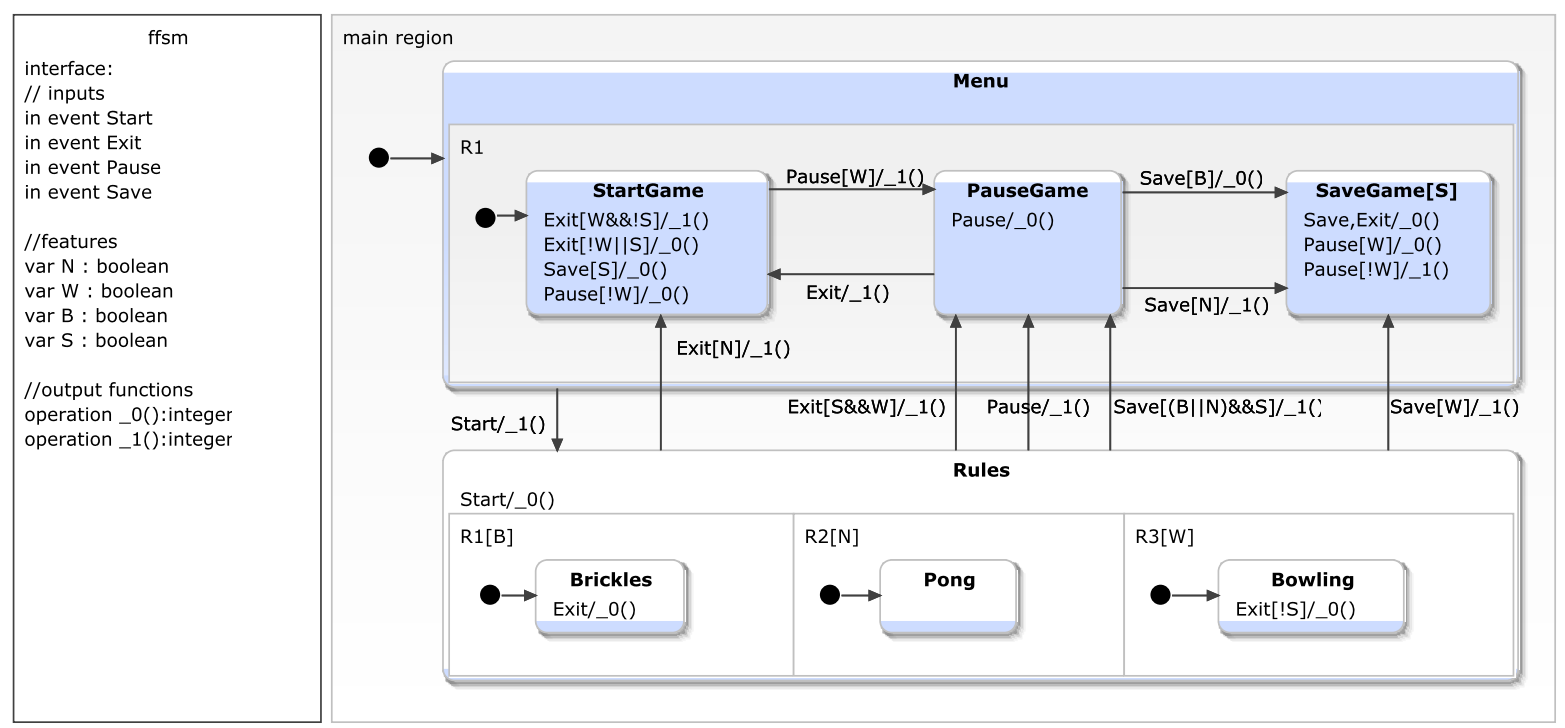

Figure 30 - HFSM for AGM SPL on the implemented tool.

Public Licence - LGPL) and the Z3 SMT Solver (MOURA; BJØRNER, 2008) (MIT licence). The Z3 tool include a proper SAT Solver that we use in our approach to check feature constraints. One extra plug-in was added to the existing set of Yakindu plug-ins, and 15 new classes with a total of 44 new functions were implemented. Some Yakindu classes were extended for the validation of graphical elements and test generation. The main graphical editor was changed to support basic elements as explained in Example 20. ConFTGen supports modeling, validation, derivation, and test-case generation of HFSMs. Figure 30 shows the HFSM for AGM in the tool that is equivalent to the HFSM presented in Figure 25.

We used the Java language to implement parsers and process HFSMs and generate the semantic FFSM. We represent the FFSM in a .txt file that is processed by another Java parser. Figure 31 shows the FFSM generated with 26 transitions from the HFSM that is equivalent to the FFSM presented in Figure 20. In this adapted format only transitions are described as:

“source@z3condition -- input@z3condition/output - > target@z3condition”

The source of the first transition is the root state.

\subsubsection{Model Derivation}

Once the HFSM is modeled the configuration file can be used to select product configurations for AGM. Using the product configuration $\rho_{5}$ that is equivalent to $W \wedge \neg S$ (Example 6) the tool can derive a pruned HFSM. Figure 32 shows the reduced HFSM with only satisfiable elements and Figure 33 shows the semantic FFSM resultant from the reduced HFSM. 


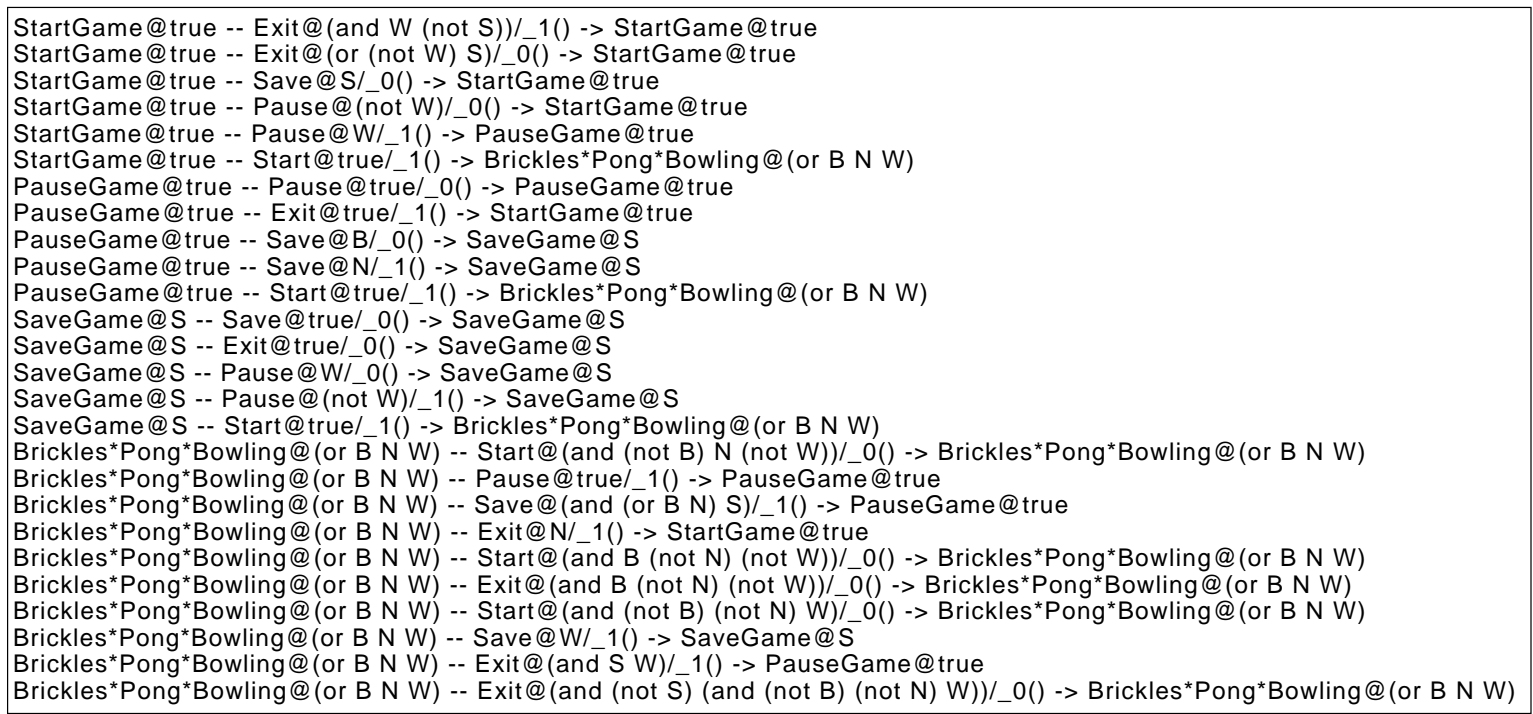

Figure 31 - Textual FFSM for AGM SPL on ConFTGen.

interface:
// inputs
in event Start
in event Exit
in event Pause
in event Save
//features
var $\mathrm{N}$ : boolean
var W : boolean
var $\mathrm{B}$ : boolean
var S : boolean
//output functions
operation_0():integer
operation_1():integer

Figure 32 - Derived HFSM for AGM SPL. 

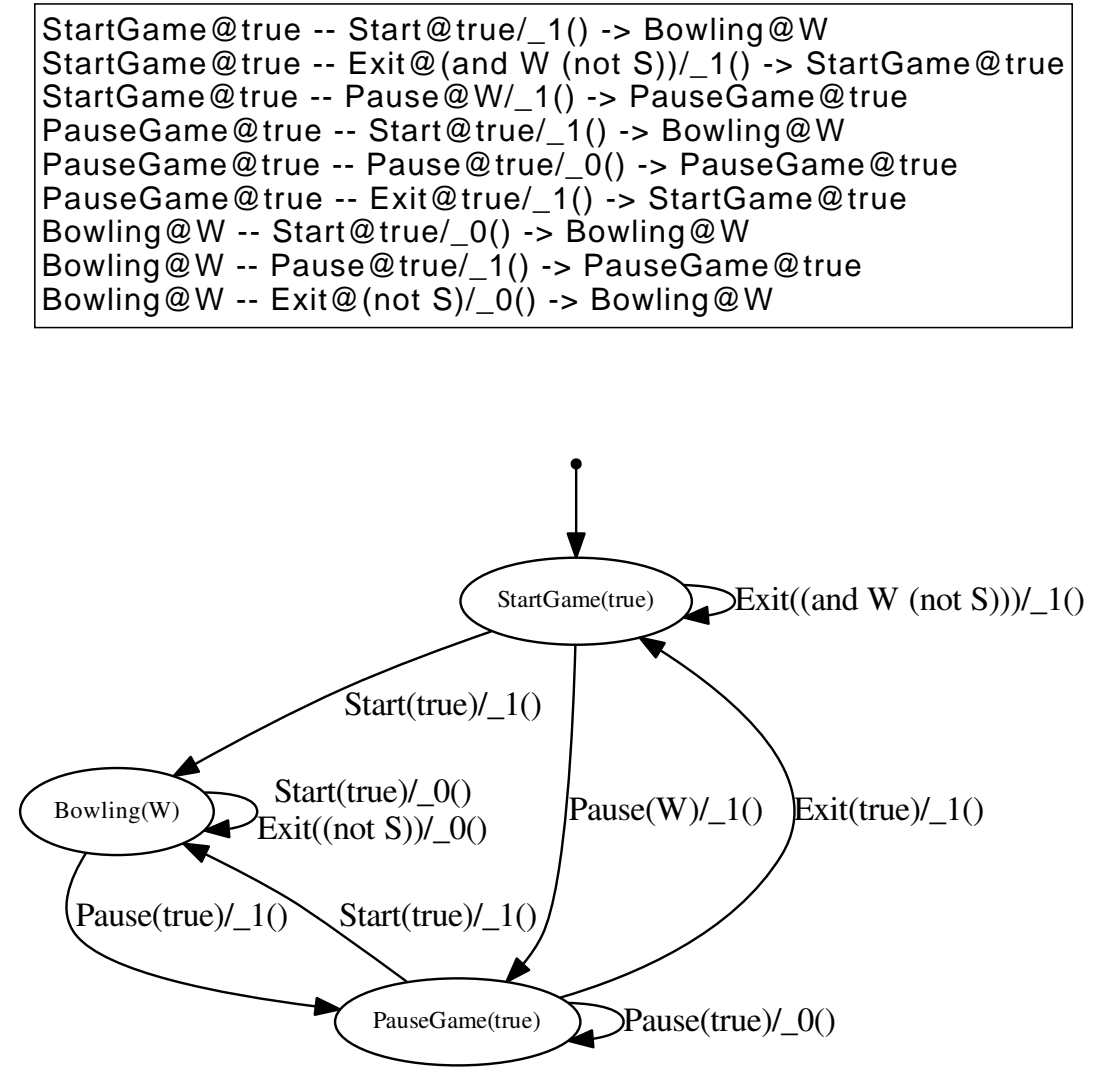

Figure 33 - Semantic FFSM of the derived HFSM.

Next, we present how SAT Solvers are used in model validation and followed by checking prefix and equivalence relations based on feature constraints.

\subsubsection{Model Validation}

We also use the Z3 tool (MOURA; BJØRNER, 2008) to check propositional formulas for FFSM properties. First, we perform the syntax checking on the HFSM from Definition 5.1.6 item 8 and Definition 5.1.12 item 4. Then, the FFSM model is checked syntacticaly and semanticaly.

To check the initial FFSM conditions on the FFSM of Figure 20, we: (i) transform the feature model of Figure 10 into a propositional formula; and (ii) generate assertions to check feature constraints of conditional states and transitions. Subsequently, we check the validity conditions on the generated propositional formula.

In Z3, push and pop commands are used to temporarily set the context (e.g., with assertions), and once a verification goal is discharged the context can be reset. The (check - sat) command is used to evaluate the assertions which return sat or unsat. If a conditional state check yields unsat, then there is no product that will ever have this 


\begin{tabular}{|l|}
\hline (define-sort Feature () Bool) \\
(declare-const G Feature) \\
(declare-const A Feature) \\
(declare-const M Feature) \\
(declare-const L Feature) \\
(declare-const C Feature) \\
(declare-const R Feature) \\
(declare-const B Feature) \\
(declare-const N Feature) \\
(declare-const W Feature) \\
(declare-const V Feature) \\
(declare-const Y Feature) \\
(declare-const P Feature) \\
(declare-const S Feature)
\end{tabular}

(a)

\begin{tabular}{|l|} 
(assert (and \\
$\mathrm{G}$ \\
$(=\mathrm{A} \mathrm{G})$ \\
$(=\mathrm{M} \mathrm{A})$ \\
$(=\mathrm{L} \mathrm{A})$ \\
$(=\mathrm{C})$ \\
$(=\mathrm{R} \mathrm{G})$ \\
$(=($ or $B \mathrm{~N} \mathrm{~W}) \mathrm{R})$ \\
$($ not $($ and $\mathrm{B} \mathrm{N}))$ \\
$($ not $($ and $\mathrm{B} \mathrm{W}))$ \\
$($ not $($ and N W $))$ \\
$(=\mathrm{V} \mathrm{G})$ \\
$(=\mathrm{Y} \mathrm{V})$ \\
$(=\mathrm{P} \mathrm{V})$ \\
$(=>\mathrm{S} \mathrm{V})$ \\
)$)$
\end{tabular}

(b)

\begin{tabular}{|l|}
\hline (push) \\
(assert (or B N W)) \\
(check-sat) \\
(pop) \\
(push) \\
(assert S) \\
(check-sat) \\
(pop) \\
$\cdot$ \\
$\cdot$ \\
RESULT \\
sat \\
sat \\
\hline
\end{tabular}

(c)

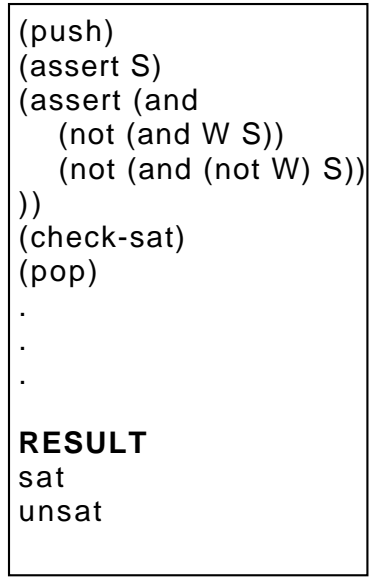

(d)

Figure 34 - SMT parts for checking conditional states and the complete property.

state and hence, the FFSM is invalid.

Example 26. Figure 34 presents parts of the generated SMT files used for checking the validity of conditional states and completeness property from the FFSM presented in Figure 20, where: (a) all features are declared as Boolean variables: (b) the feature model propositional formula is asserted which represent all feature relations; (c) conditional states Brickles * Pong * Bowling $[B\|N\| W]$ and Save $[S]$ are verified; and (d) a completeness check on the conditional state Save $[S]$ for input Pause is verified (see Figure 20). To check conditional states we combine and execute parts (a), (b), and (c), while to check completeness (a), (b), and (d) are combined and executed. In the end, for every (check-sat) command we have an answer (sat or unsat) that we automatically connect back to our tool.

To check completeness, we select a conditional state and then select a conditional input and check whether it exists in every product configuration that the state exists. First, we assert the constraint of the conditional state to use only satisfiable configurations represented by $\Lambda_{s}$. Then, assume that two transitions $t_{a}, t_{b}$ leave the selected conditional state (may be several transitions). For each transition we combine the input and target feature constraints into $\omega_{a}$ and $\omega_{b}$, where $\Lambda_{a} \subseteq \Lambda$ is the subset of configurations that the constraint $\omega_{a}$ can satisfy, and $\Lambda_{b} \subseteq \Lambda$ for $\omega_{b}$. Then, we want to check whether $\Lambda_{s} \subseteq \Lambda_{a} \cup \Lambda_{b}$, but using an inverted logic. By negating the elements of the last assertion if $\Lambda_{s} \cap\left\{\Lambda \backslash \Lambda_{a} \cap \Lambda \backslash \Lambda_{b}\right\}=\varnothing$ (unsat) then we have $\Lambda_{s} \subseteq \Lambda_{a} \cup \Lambda_{b}$ which is cheaper to check in terms of execution time.

For the minimal and initially connected properties we use similar checks for whether there are enough separating sequences and reaching paths, respectively. Only for 


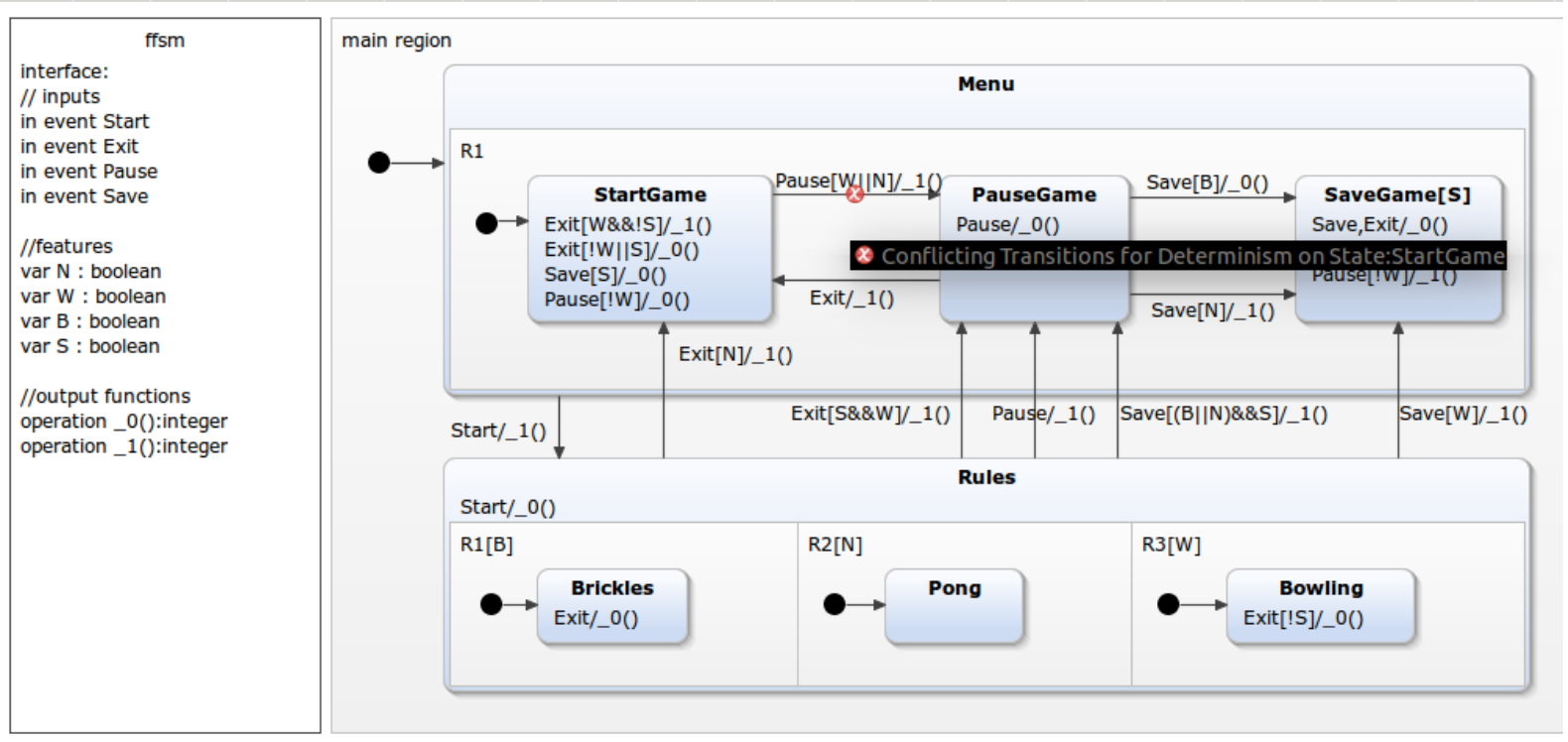

Figure 35 - HFSM for AGM SPL with deterministic error.

determinism we check whether every pair of transitions with the same input does not have an intersection of configurations on leaving transitions, i.e., $\Lambda_{a} \cap \Lambda_{b}=\varnothing$ (unsat). Figure 35 shows an example of deterministic error when we change the feature constraint $W$ for $(W \| N)$ of a transition from StartGame to PauseGame that enter in conflict with the self-loop transition of StartGame with Pause input and $\neg W$ feature constraint. The conflict occurs due to the non-empty intersection of two product configurations that has $N$ which contain both transitions resulting non-determinism.

\subsubsection{Prefix Relation Check}

The $\mathrm{Z} 3$ tool is also used for checking conditional prefixes and equivalence relations for a pair of conditional tests $\left(\delta_{a}, \omega_{a}\right),\left(\delta_{b}, \omega_{b}\right) \in I^{*} \times B(F)$, where $\Lambda_{a} \subseteq \Lambda$ is the subset of configurations that the constraint $\omega_{a}$ can satisfy, and $\Lambda_{b} \subseteq \Lambda$ for $\omega_{b}$. To check whether $\left(\delta_{a}, \omega_{a}\right)$ is a conditional prefix of $\left(\delta_{b}, \omega_{b}\right)$, we perform the following steps:

1. If $\delta_{a}$ is a prefix of $\delta_{b}$, then we assert the feature constraint $\chi$ of the feature model $F M=(F, \chi, \Lambda)$ and the logical conjunction of feature constraints $\left(\omega_{a} \wedge \omega_{b}\right)$. If it returns unsatisfiable, then there is no common configuration between both constraints $\Lambda_{a} \cap \Lambda_{b}=\varnothing$ and we cannot derive/deduce the prefix relation.

2. If the first check returns satisfiable, then, another check identify the prefix relation $\Lambda_{a} \subseteq \Lambda_{b}$. We assert the first condition $\omega_{a}$ followed by the negation of the second $\neg \omega_{b}$ to check whether $\Lambda_{a} \cap\left(\Lambda \backslash \Lambda_{b}\right)=\varnothing$ is unsatisfiable. If the proposition turns out to be satisfiable, then there are configurations for $\omega_{a}$ which $\neg \omega_{b}$ was not able to 


\begin{tabular}{|l|}
\hline (define-sort Feature () Bool) \\
(declare-const G Feature) \\
(declare-const A Feature) \\
(declare-const M Feature) \\
(declare-const L Feature) \\
(declare-const C Feature) \\
(declare-const R Feature) \\
(declare-const B Feature) \\
(declare-const N Feature) \\
(declare-const W Feature) \\
(declare-const V Feature) \\
(declare-const Y Feature) \\
(declare-const P Feature) \\
(declare-const S Feature)
\end{tabular}

(a)

\begin{tabular}{|l|}
\hline (assert G) \\
(assert (and \\
$(=A G)$ \\
$(=M A)$ \\
$(=L A)$ \\
$(=C G)$ \\
$(=R G)$ \\
$(=($ or $B N W) R)$ \\
$($ not $($ and $B N))$ \\
$($ not $($ and $B \mathrm{~W}))$ \\
$($ not $($ and $N \mathrm{~W}))$ \\
$(=V G)$ \\
$(=Y V)$ \\
$(=P V)$ \\
$(=>S V)$ \\
)$)$
\end{tabular}

(b)

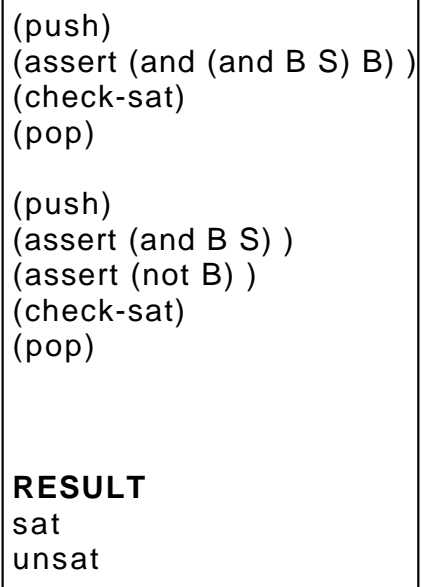

(push)

(assert (and (and B S) B) ) (check-sat) (pop)

(push)

(assert (and B S) )

(assert (not B))

(check-sat)

(pop)

\section{RESULT}

sat

unsat

Figure 36 - SMT parts for checking a conditional prefix relation.

invalidate, i.e., $\Lambda_{a} \nsubseteq \Lambda_{b}$. Otherwise, when the result is unsatisfiable, then, $\neg \omega_{b}$ was able to invalidate all configurations of $\omega_{a}$, i.e., $\Lambda_{a} \subseteq \Lambda_{b}$.

Example 27. Figure 36 presents parts of the generated SMT file used to check whether a conditional test $\left(\delta_{a}, \omega_{a}\right)=(a,(B \wedge S))$ is a conditional prefix of $\left(\delta_{b}, \omega_{b}\right)=(a a,(B))$, where: (a) all features are declared as Boolean variables, (b) the feature constraint of the feature model is asserted, and (c) the prefix relation checks are asserted. In Z3, push and pop commands are used to temporarily set the context (e.g., with assertions), and once a verification goal is discharged the context can be reset. The (check - sat) command is used to evaluate the assertions which return sat or unsat. First, we notice that $a$ is a prefix of $a a$, then, checking the conjunction of both conditions $((B \wedge S) \wedge B) \equiv(B \wedge S)$ results in sat. Next, we assert the first constraint $(B \wedge S)$ and also assert the negation of the second constraint $\neg(B)$ to eliminate configurations of the first, which results in unsat. Thus, $(a,(B \wedge S))$ is a conditional prefix of $(a a,(B))$.

The Z3 tool is also used for checking feature constraints in most definitions in this thesis. The usage is a variation of the Example 27 where we change the (c) part of the SMT file. Given a feature constraint $\varphi \in B(F)$ that requires checking, we use (assert $\varphi$ ) in (c) to check if it returns sat or unsat.

\subsubsection{Test Generation}

To generate a configurable test-suite for the HFSM presented in Figure 30 we use its semantic FFSM presented in Figure 31. Following the test design approach on Section 4.4 we obtained:

- Configurable state cover set: 3 tests and size 7; 
- Configurable transition cover set: 17 tests and size 60;

- Complete configurable test-suite: 31 tests and size 132.

Once we derive the reduced HFSM for AGM product $\rho_{5}$ of Figure 32 we have two options:

1. We can instantiate the configurable test-suite for $\rho_{5}$; or

2. We can use a derived FSM from the HFSM of Figure 32 to generate a test-suite using the classic HSI method.

For the first option, we have a test-suite with 12 tests with size 50, while the second option 11 tests with size 42 . If we instantiate all valid products using the product-by-product approach, we would have 43 new tests with total size 203. Thus, instead of generating tests individually, we can use the FFSM to generate a configurable test-suite, and then instantiate individually, potentially saving $35 \%$ of test effort.

Following the incremental approach of our first line of research was found similar results to the FFSM approach. The final test set created incrementally for all valid configurations had 24 tests with size 123. However, one fundamental difference is the number of times that we had to execute the test-case generation algorithm and process a test-suite before incrementing it. According to our experimental results, our first line of research favors the testing of few SPL products while the second line of research favors when we have a large number of valid configurations that require testing.

\subsection{Concluding Remarks}

In this chapter, we presented the Configurable Full Test Generator (ConFTGen) test design tool. The ConFTGen has graphical support based on the Eclipse platform that makes possible the integration of several related tools to build chain tools and ease the automation of test design. Also, we presented how SAT Solvers are used in the validation and derivation of tests artifacts. 
CHAPTER

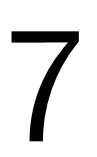

\section{EXPERIMENTAL STUDIES}

In this chapter, we present experimental studies for both directions of research for test design of SPLs presented in this thesis. Section 7.1 presents an experiment for the IRT-SPL strategy, while Section 7.2 presents random FFSM models for the CFC-SM approach. Finally, Section 7.3 shows a case study for the automotive industry where configurable test artifacts are developed with the aid of the ConFTGen tool.

\subsection{Incremental Regression-based Approach}

To evaluate the applicability of IRT-SPL and the reuse efficiency of our selection algorithm, we conducted a study to compare the effort required to test new products to other test-case reuse strategies. Our research question is: How much test effort can be saved using IRT-SPL to test a set of new SPL products compared to existing test-case reuse strategies?

\subsubsection{Experimental Setup}

The setup of our experiment consists of designing several SPL products in different orders and comparing the total effort required to test all products. We compare the effort of IRT-SPL to other reuse strategies found in the literature. A survey on some approaches (EI-FAKIH; YEVTUSHENKO; BOCHMANN, 2004; PAP et al., 2007; CHEN; PROBERT; URAL, 2009; UZUNCAOVA; KHURSHID; BATORY, 2010; CAPELLARI et al., 2012; LOCHAU et al., 2014) showed two generic reuse strategies, which are described as follows:

1. The first reuse strategy TSPL was named after the FSM-TSPL approach from Capellari et al. (CAPELLARI et al., 2012). They only reuse test-cases of the last product for conformance testing of a new product where they increment test-cases. 


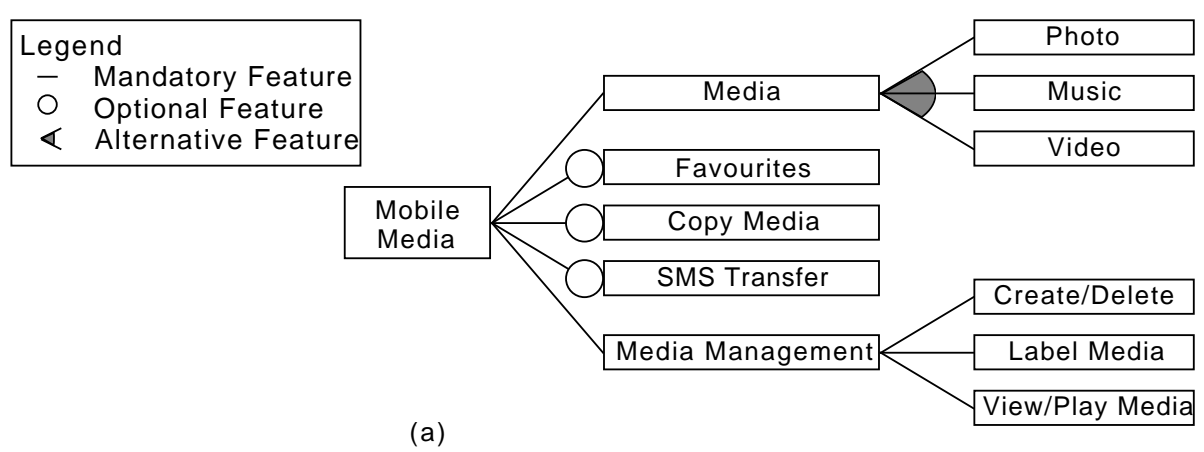

(a)

\begin{tabular}{|c|c|c||c|c||c||c||c||c|c|c|c|c|c|}
\hline Products & MP & MM & MV & F & CM & SMS & Products & MP & MM & MV & F & CM & SMS \\
\hline \hline 1 & $\mathrm{X}$ & & & & & & 13 & X & & & X & X & \\
\hline \hline 2 & & $\mathrm{X}$ & & & & & 14 & & $\mathrm{X}$ & & $\mathrm{X}$ & $\mathrm{X}$ & \\
\hline \hline 3 & & & $\mathrm{X}$ & & & & 15 & & & $\mathrm{X}$ & $\mathrm{X}$ & $\mathrm{X}$ & \\
\hline \hline 4 & $\mathrm{X}$ & & & $\mathrm{X}$ & & & 16 & $\mathrm{X}$ & & & & $\mathrm{X}$ & $\mathrm{X}$ \\
\hline \hline 5 & $\mathrm{X}$ & & & & $\mathrm{X}$ & & 17 & & $\mathrm{X}$ & & & $\mathrm{X}$ & $\mathrm{X}$ \\
\hline \hline 6 & $\mathrm{X}$ & & & & & $\mathrm{X}$ & 18 & & & $\mathrm{X}$ & & $\mathrm{X}$ & $\mathrm{X}$ \\
\hline \hline 7 & & $\mathrm{X}$ & & $\mathrm{X}$ & & & 19 & $\mathrm{X}$ & & & $\mathrm{X}$ & & $\mathrm{X}$ \\
\hline \hline 8 & & $\mathrm{X}$ & & & $\mathrm{X}$ & & 20 & & $\mathrm{X}$ & & $\mathrm{X}$ & & $\mathrm{X}$ \\
\hline \hline 9 & & $\mathrm{X}$ & & & & $\mathrm{X}$ & 21 & & & $\mathrm{X}$ & $\mathrm{X}$ & & $\mathrm{X}$ \\
\hline \hline 10 & & & $\mathrm{X}$ & $\mathrm{X}$ & & & 22 & $\mathrm{X}$ & & & $\mathrm{X}$ & $\mathrm{X}$ & $\mathrm{X}$ \\
\hline \hline 11 & & & $\mathrm{X}$ & & $\mathrm{X}$ & & 23 & & $\mathrm{X}$ & & $\mathrm{X}$ & $\mathrm{X}$ & $\mathrm{X}$ \\
\hline \hline 12 & & & $\mathrm{X}$ & & & $\mathrm{X}$ & 24 & & & $\mathrm{X}$ & $\mathrm{X}$ & $\mathrm{X}$ & $\mathrm{X}$ \\
\hline
\end{tabular}

(b)

Figure 37 - Mobile Media feature model and derived products.

2. The second reuse strategy DIATP was named after the Delta-oriented Incremental Architecture-based Testing Process found in the approach of Lochau et al. (LOCHAU et al., 2014). They reuse test-cases of all previous products selecting test-cases to verify the unchanged behavior of the new product, but they generate new test-cases for the changed behavior without the increment of test-cases.

To obtain a fair comparison on the effort of our strategy, TSPL, and DIATP we setup similar environments also using the P method for TSPL and DIATP.

The embedded camera Mobile Media SPL (FIGUEIREDO et al., 2008) was used to compare all three reuse strategies. The Mobile Media SPL contains several features, such as photo manipulation, music, and videos on mobile devices which results in total 56 product configurations where we selected 24 relevant product configurations. Figure 37 (a) presents the feature diagram with three Or features (Photo(MP), Music(MM), and Video(MV)) and three optional (Favourites(F), Copy Media(CM), and SMS Transfer(SMS)) used to characterize all possible configurations of the SPL. Figure 37 (b) presents 24 configurations of Mobile Media used to design corresponding products. Note that each product of the order 1 to 24 increases the number of features compared to the previous products.

For each product, an FSM was modeled with varying number of states from three to six, and with fixed eight inputs and two outputs. All FSMs share some properties: 


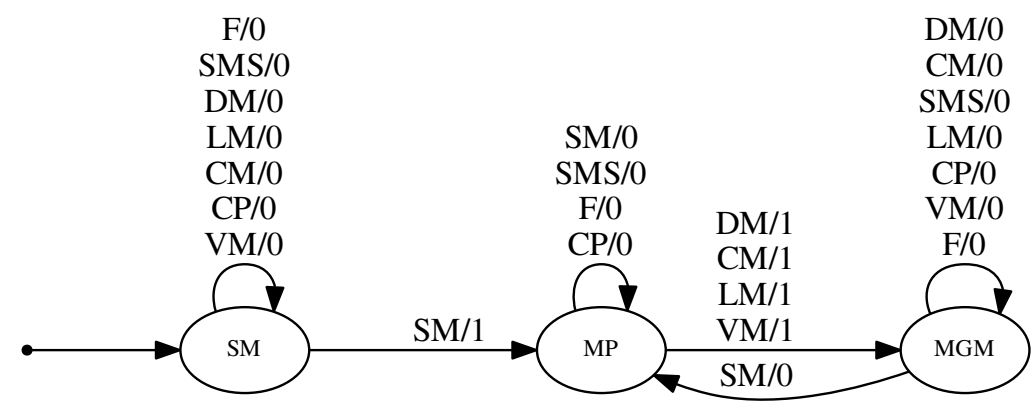

Figure 38 - FSM of the first product of Mobile Media.

complete, deterministic, minimal, and initially connected. Figure 38 shows the abstract FSM of the first product.

\subsubsection{Analysis of Results and Discussion}

The collected data from the experiments is shown in Figure 39. The variable $x$ is the manual concretization value $(x=200$ (UTTING; LEGEARD, 2006; Graham D., 2012)) from the effort formula effort $=($ concrete $* x)+$ execution presented in Section 3.1. On (a) and (b) we have the derivation of products with an increasing number of features when $x=10$ and $x=100$, respectively. We pick values 10 and 100 to check how it scales for low and high values of $x$. On (c) and (d) we have the derivation of products with decreasing number of features when $x=10$ and $x=100$, respectively. Finally, on (e) and (f) we have the derivation of products with a random number of features when $x=10$ and $x=100$, respectively.

We noticed that the total effort required to test the Mobile Media SPL varies according to the value $x$. First, noticed that for our case study the effort saving percentage when $x=100$ and $x=200$ is approximately the same and when $x$ is below 10 our approach does not provide significant saving. Also, the number of newly designed products should be considered as for few products there is no significant difference of effort bellow 4 product configurations. Moreover, only a few design product random orders were considered and relatively small feature models. These are threats to the validity of this study.

Our approach assumes new products to be similar to old ones. In the worst case scenario, the new product to be tested is completely different from any product developed before. However, in the SPL approach, this is unlikely as commonalities are propagated throughout the entire family.

In summary, we conclude that our approach provides effort savings starting from $5 \%$ when we have more than 4 product configurations and the $x$ value is over 10 and goes 
Effort by Product when $\mathrm{x}=10$

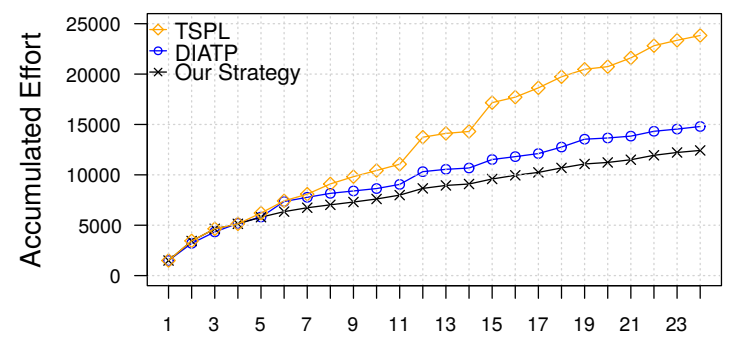

(a) Design Product Order

Effort by Product when $x=10$

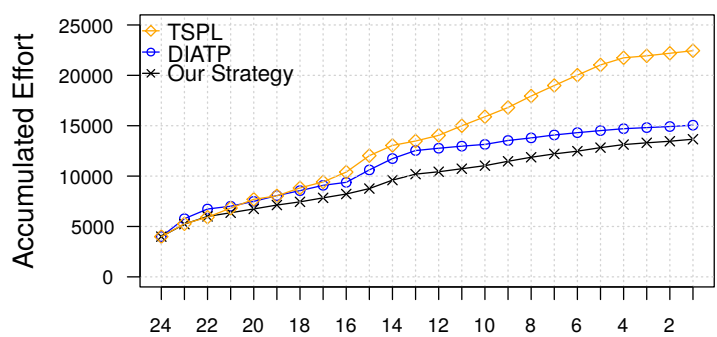

(c) Design Product Order

Effort by Product when $\mathrm{x}=10$

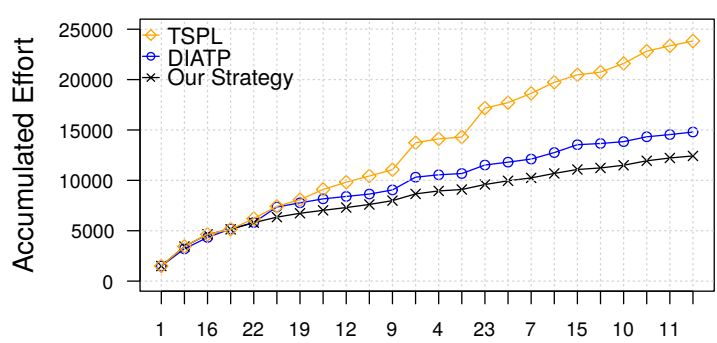

(e) Design Product Order
Effort by Product when $x=100$

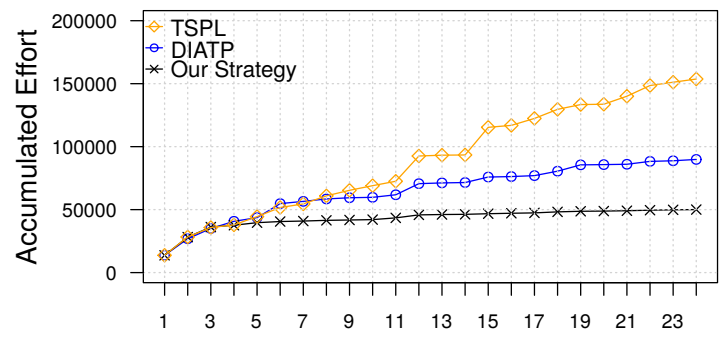

(b) Design Product Order

Effort by Product when $x=100$

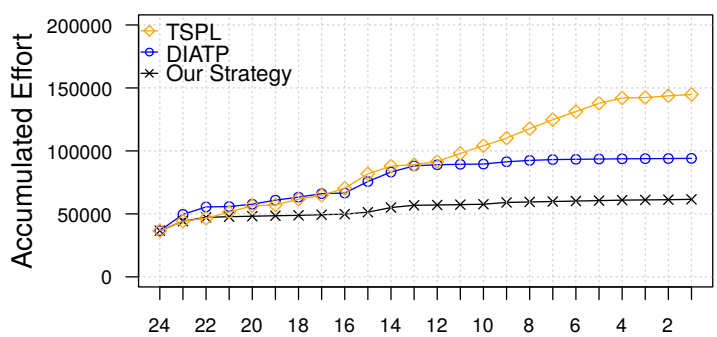

(d) Design Product Order

Effort by Product when $\mathrm{x}=100$

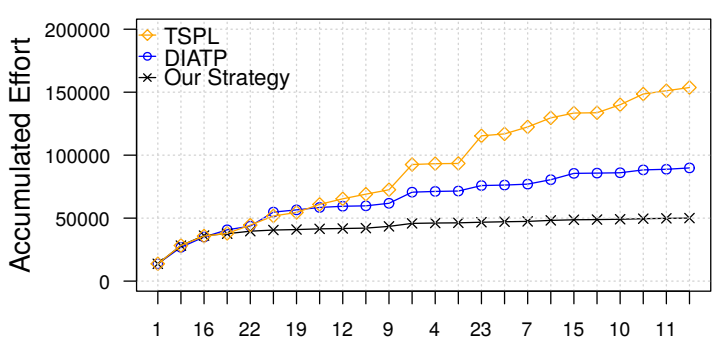

(f) Design Product Order

Figure 39 - Accumulated effort per designed product.

up to $36 \%$ when we have 24 product configurations, and the $x$ value is over 100 compared to other test reuse strategies.

The random order the time to execute every strategy depends on the complexity of the $\mathrm{P}$ method. Traditional test-case generation methods for the full fault coverage that use FSMs as test models (e.g. W (CHOW, 1978), HSI (LUO et al., 1994)) are not incremental. Thus, they cannot increment test-cases for new specifications based on old test-cases to improve reuse. However, generated new test-cases can be compared to old test-cases for reuse resulting in a weaker reuse strategy as some of these new test-cases may be equivalent to existing old test-cases.

Our experiment has been limited mostly to flat FSMs with few states and few new products. One of the issues regarding the $\mathrm{P}$ method is the increasing time required to 
generate test-cases based on the number of states times inputs plus the size of the test input set to start with. Both of these issues (few subjects and time) are threats to the validity of our results for real-world cases. We plan to mitigate these threats by analyzing some realistic case studies as a benchmark for our future research. Realistic FSMs use hierarchy to sustain scalability. Thus, an extension of FSM-based test generation methods for full fault coverage is required.

\subsection{Random Featured-based Test Model}

To evaluate the applicability and the efficiency of our approach, we conducted an experiment to evaluate random FFSMs and random feature models. We focus on the characteristics of the models, the comparison of test-suite size and test generation time against the product-by-product approach.

Focusing on the full fault coverage, we are aware of some incremental test-case generators for FSMs (EI-FAKIH; YEVTUSHENKO; BOCHMANN, 2004; PAP et al., 2007; SIMAO; PETRENKO, 2010). These methods can process an existing test set and increment few tests for a new product configuration. Despite the good results on the few number of new tests, these methods have to process the test set for every new product configuration. Moreover, they are quite sensitive to the size of the test set used as input. Hence, they may not scale well to increment large test sets for large specifications.

We aimed at answering the following research questions:

\section{General Results}

- Q1- Is there a difference between generating a test-suite for an FSM and pruning a configurable test-suite for the same FSM?

- Q2- In which scenario do we reduce the number of test-cases using an FFSM instead of FSMs?

- Q3- In which scenario do we have smaller test generation times using an FFSM instead of FSMs?

Feature Model Influence

- Q4- Is there a relation between the feature model and the number of conditional transitions?

- Q5- Is there a relation between the feature model and the configurable test-suite size? 
- Q6- Is there a relation between the feature model and the extended HSI test generation time?

\subsubsection{Experimental Setup}

Our experiment aims at generating test-suites (full fault coverage) for random FFSMs and random feature models. The adaptable parameters (independent variables) include random feature models that are generated with the following parameters (SEGURA et al., 2012):

- Number of features (20);

- Percentage of custom constraints (0);

- Probability of a feature being mandatory (0);

- Probability of a feature being optional (33);

- Probability of a feature being in an or-relation (33);

- Probability of a feature being in an alternative-relation (33);

- Maximal branching factor (10);

- Maximal number of children in a set relationship (10).

We decided to use no custom constraints and mandatory features. Custom constraints may be redundant to constraints defined by the feature structure. Mandatory features do not alter the number of product configurations, and we do not want to have models which too many mandatory constraints. The maximal branching factor limits the number of branches in the feature model structure. The number of children in a set relationship limits the number of features that may be in an alternative/or/optional subset relation.

To visualize some characteristics for the relation/influence of feature models and FFSMs we divided 20 random feature models into two groups. The first group has feature models with less than the median of independent features. The second group has feature models with more than the median of independent features. An independent feature is placed in an or-relation or set in parallel with other optional features, which makes the number of product configurations grow exponentially.

To answer our research questions we executed three groups of FFSMs. Each group has 20 random FFSMs and 20 random feature models. The FFSM groups are:

1. FFSMs with 12 conditional states, 10 to 15 inputs/outputs and 100-450 transitions.

2. FFSMs with 18 conditional states, 15 to 20 inputs/outputs and 225-800 transitions. 
3. FFSMs with 24 conditional states, 20 to 25 inputs/outputs and 400-1250 transitions.

Each random FFSM is generated by randomizing the target state of conditional transitions. We assume that only $1 / 3$ of the conditional states and transitions have random feature constraints. Thus, $2 / 3$ of the behavior is part of the core specification. We use FFSMs that are deterministic, initially connected, and minimal.

The fixed parameters (controlled variables) are:

- Each random FFSM is linked (for feature mapping) to one random feature model;

- The number of inputs and outputs are the same as the number of FFSM states;

- One derived FSM (from the FFSM) represents the core specification of the SPL;

- The test generation method HSI used for FSM and FFSMs.

Then, we measure (dependable variables):

1. The number of new different tests: we measure the number of new tests that are required to test all products using an FFSM-based test-suite against 20 random products with individual FSM-based test-suites;

2. Test-suite size for the FSM core: we measure the size of the test-suite pruned for the FSM core specification from an FFSM-based test-suite against generating the test-suite directly from the FSM core specification;

3. Checking and generation time: we measure the amount of time it takes to generate tests for an entire FFSM against one FSM and a set of FSMs.

The running environment used Ubuntu 15.04 (64 bit) operating system on an Intel processor i7-5500U at $2.40 \mathrm{GHz}$ with $12 \mathrm{~GB}$ of RAM.

\subsubsection{Analysis and Threats to Validity}

The collected data in our experiments is analyzed below.

Q1- Is there a difference between generating a test-suite for an FSM and pruning a configurable test-suite for the same FSM?

To answer this question, we generated complete configurable test-suites for all 3 groups of FFSMs. As stated before, each group has 20 random FFSMs where each group has larger FFSM specifications than the previous group. For each FFSM we derived an FSM which represents the SPL core specification (core product). First, we generated a 

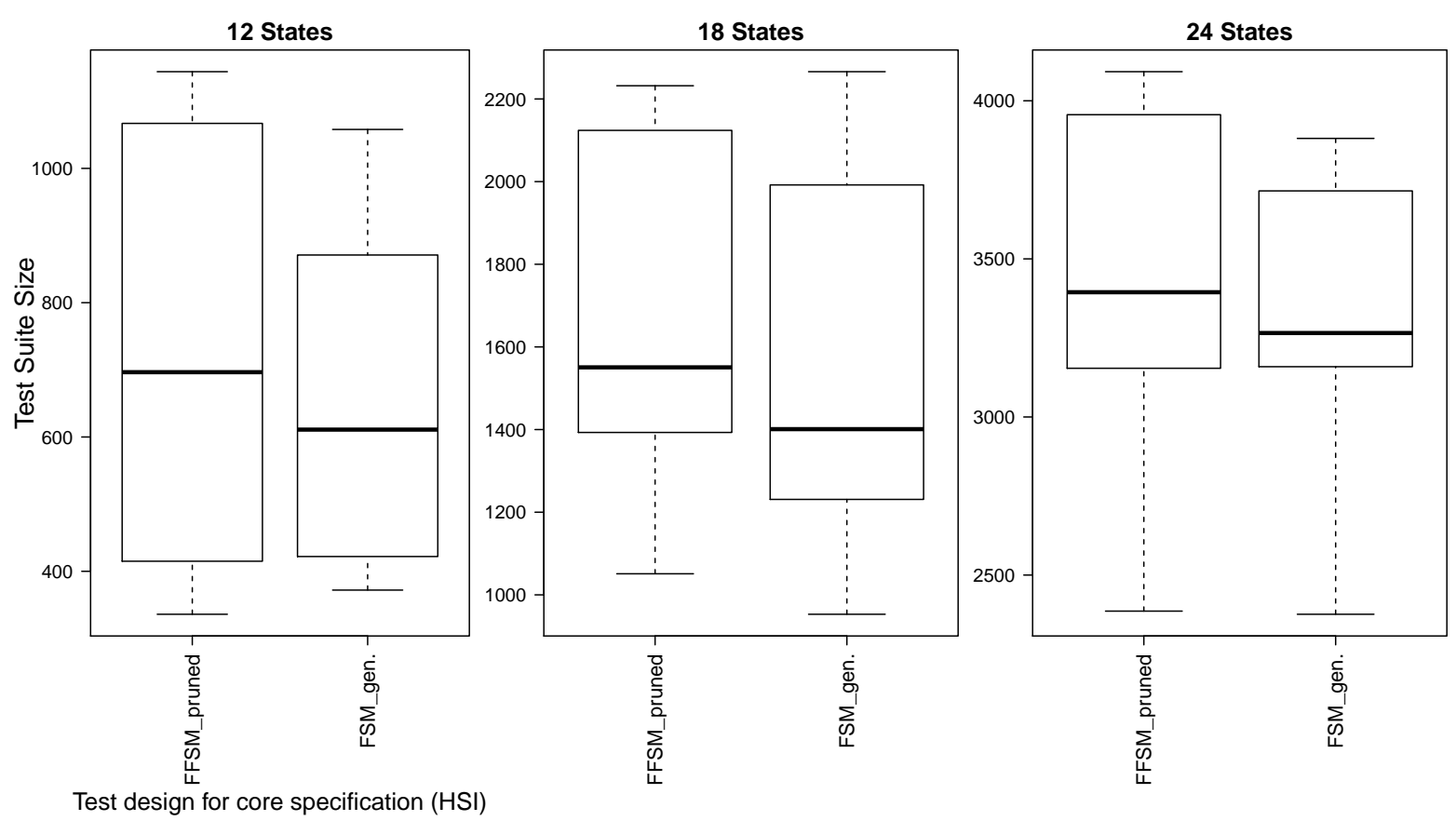

Figure 40 - Test-suite size of the core specification.

complete configurable test-suite $C T C$ for an FFSM $F F$ using the extended HSI method presented in this thesis, then we pruned $C T C$ for the core product. Second, we generated a complete test-suite for the core product using the HSI method. Figure 40 shows the results on the test-suite size for each case. The first group of FFSMs with 12 conditional states shows the tests suite sizes for the pruned case (FFSM-pruned) and the directly generated from the core product (FSM_gen).

We noticed that the results from the first FFSM group continue in the other two groups as it increases the FFSM size. There is a small difference favoring the direct application of the HSI on the core product over pruning a configurable test-suite from the extended HSI method for the same core product. Thus, our experiment does not indicate a statistically meaningful difference between generating a test-suite for an FSM and pruning a configurable test-suite for the same FSM.

\section{Q2- In which scenario do we reduce the number of test-cases using an FFSM instead of FSMs?}

To answer this question, we generated complete configurable test-suites for all 3 groups of FFSMs. As stated before, each group has 20 random FFSMs where each group has larger FFSM specifications than the previous group. For each FFSM we derived an FSM which represents the SPL core specification (core product) and then we selected 20 random valid configurations and derived an FSM for each of them. First, we generated a complete configurable test-suite $C T C$ for an FFSM FF using the extended HSI method presented in this thesis. Second, we generated a complete test-suite for the core product using the HSI method. Third, we generated a complete test-suite for each of the 20 random 

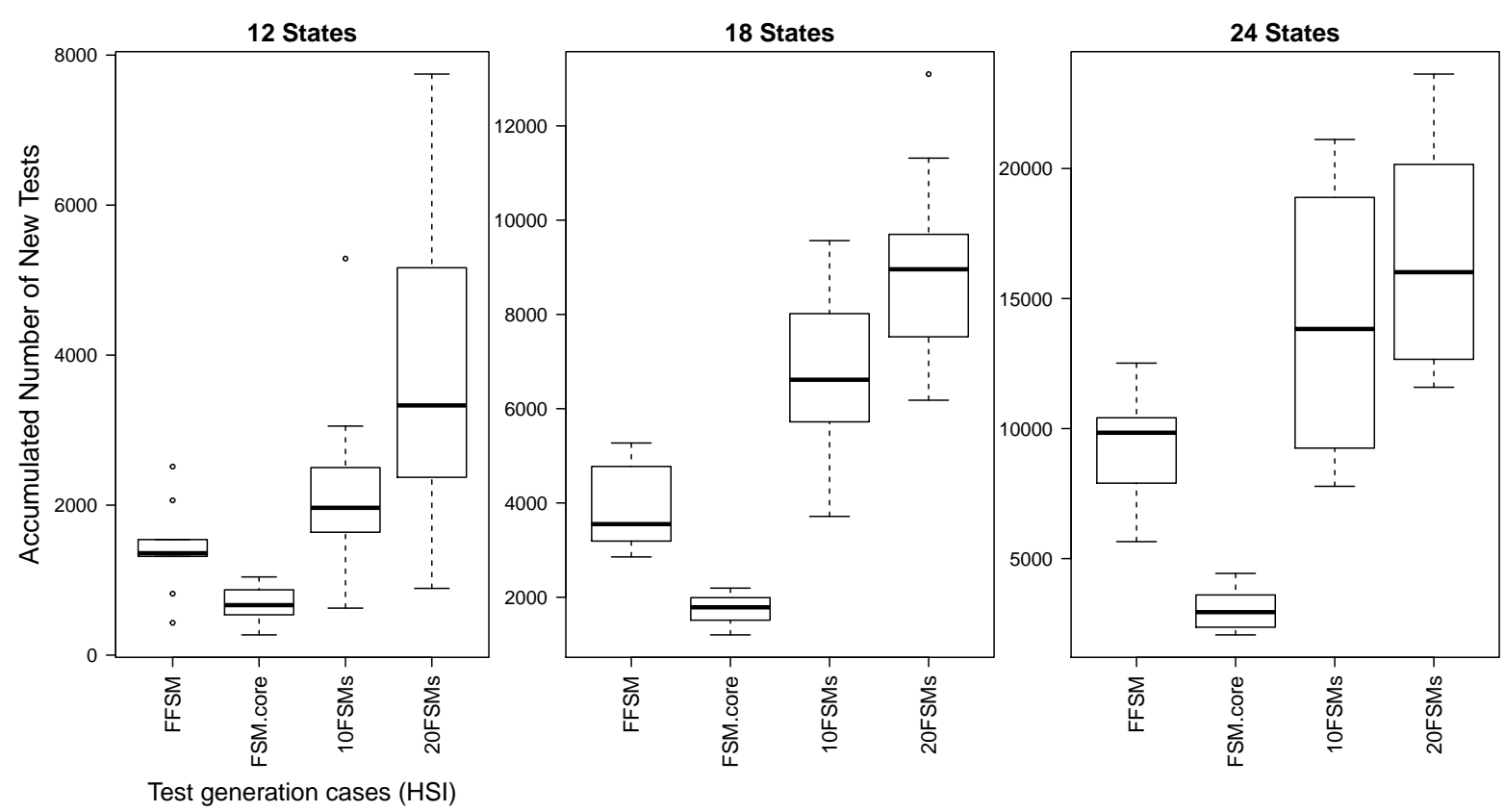

Figure 41 - Number of new tests for an FFSM and FSMs.

derived FSMs using the HSI method, and then we merged all test-suites into a single accumulated set of new different test-cases. Figure 41 shows the results on the accumulated number of new tests for each case. The first result (FFSM) shows the first case where we have the configurable test-suite size which is valid for an entire SPL. The second result (FSM.core) shows the second case, where we have the complete test-suite size for the core product. The third and fourth results (10FSMs and 20FSMs) shows the third case where we have the accumulated number of new different tests for 10 and 20 randomly derived FSMs, respectively.

We noticed that the results from the first FFSM group continue in the other two groups as it increases the FFSM size. There is a significant difference comparing the first and third cases as we need to test more FSMs. Some FFSMs have more than 10000 valid configurations, and for those SPLs testing more than 20 products individually, we may have more test-cases to concretize and execute than the whole FFSM. As stated before, the set of new tests for the third case does not have repeated test-cases, only new different ones. The results show an increasing difference in each FFSM group as we increase the FFSM specification size. Thus, our experiment indicates a statistically meaningful difference of approximately $50 \%$ in the number of new test-cases when we have more than 20 random valid configurations to test individually compared to an entire SPL using a configurable test-suite generated from an FFSM with more than 18 conditional states and 225 conditional transitions. 

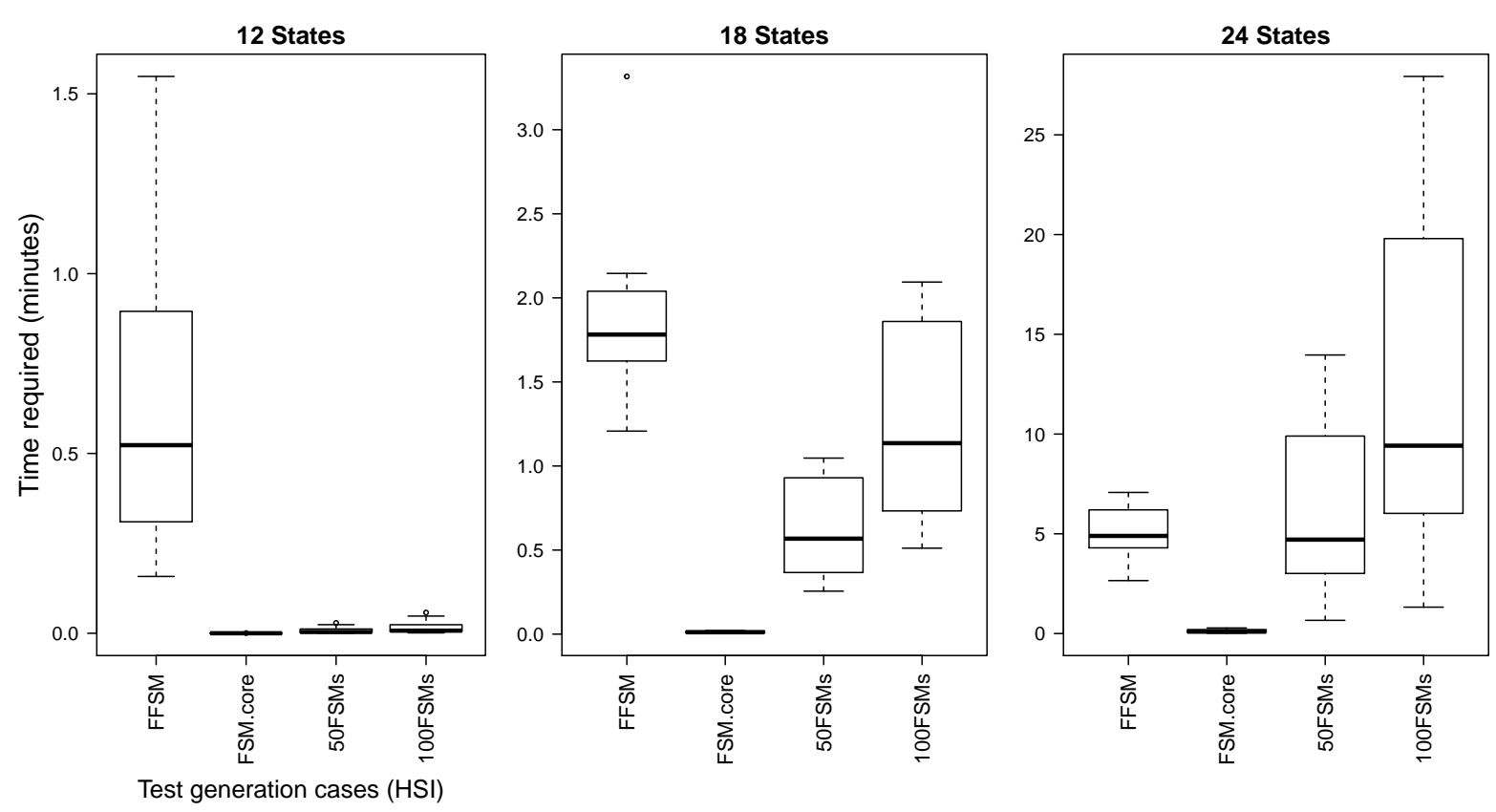

Figure 42 - Time required to execute the HSI method for one FFSM and some FSMs.

Q3- In which scenario do we have smaller test generation times using an FFSM instead of FSMs?

To answer this question, we generated complete configurable test-suites for all 3 groups of FFSMs. As stated before, each group has 20 random FFSMs where each group has larger FFSM specifications than the previous group. For each FFSM we derived an FSM which represents the SPL core specification (core product) and then we selected 100 random valid configurations and derived an FSM for each of them. First, we generated a complete configurable test-suite $C T C$ for an FFSM FF using the extended HSI method presented in this thesis and then we calculated the time required for test-case generation. Second, we generated a complete test-suite for the core product using the HSI method, and then we calculated the time required for test-case generation. Third, we generated a complete test-suite for each of the 100 random derived FSMs using the HSI method, and then we calculated the overall time required for test-case generation. Figure 42 shows the results on the overall time required for each case. The first result (FFSM) shows the first case where we have the time required to generate test-cases for the whole SPL. The second result (FSM.core) shows the second case, where we have the time required to generate a complete test-suite for the core product. The third and fourth results (50FSMs and 100FSMs) shows the third case where we have the overall time required to generate complete test-suites for all 50 and 100 randomly derived FSMs, respectively.

We noticed that the results from the first FFSM group vary on the other two groups as it increases the FFSM size. There is a significant difference comparing the first and third cases in the last FFSM group. Some FFSMs have more than 10000 valid 

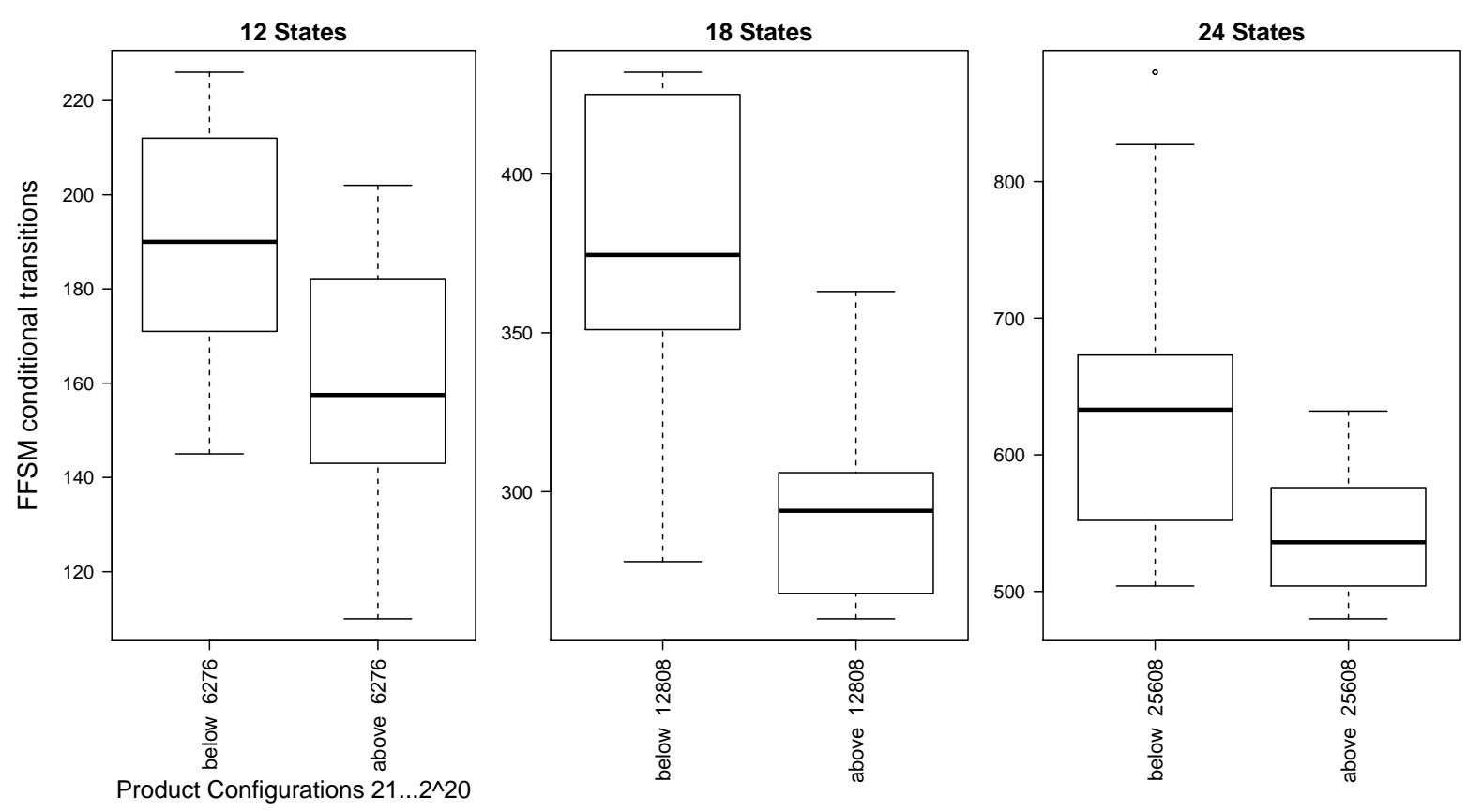

Figure 43 - Number of FFSM conditional transitions per kind of feature model.

configurations, and for those SPLs generating complete test-suites for more than 100 products individually may take more time than generating a single configurable testsuite for all 10000 valid configurations. The results show an increasing difference in each FFSM group as we increase the FFSM specification size. Thus, our experiment indicates a statistically meaningful difference of time required to generate a single configurable test-suite for an FFSM compared to generating test-suites for 100 derived FSMs when the FFSM has more than 24 conditional states and 450 conditional transitions.

Q4- Is there a relation between the feature model and the number of conditional transitions?

Figure 43 shows the results for the relation between the number of transitions of FFSMs and types of features models. For each random FFSM, we have a correspondent feature model. The results are generated for each FFSM group with 12, 18, and 24 states. In each group, we divide our 20 pairs of FFSMs and feature models in 2 subgroups of 10 each. The division was made using the median of the number of valid product configurations. The number of product configurations varies from 21 to $2^{20}$. Thus, in the first group of FFSMs with 12 conditional states the median of product configurations found from all feature models was 6276, then 12808 for the second group, and 25608 for the third group.

We noticed that the median of product configurations grows as the size of the FFSM specification grows as well. However, we believe that it was a coincidence due to the irregular distribution of valid configurations. Some feature models have 50 valid configurations while others have more than 100000 valid configurations. Thus, for the first group, we have more feature models with few valid configurations than other groups. 

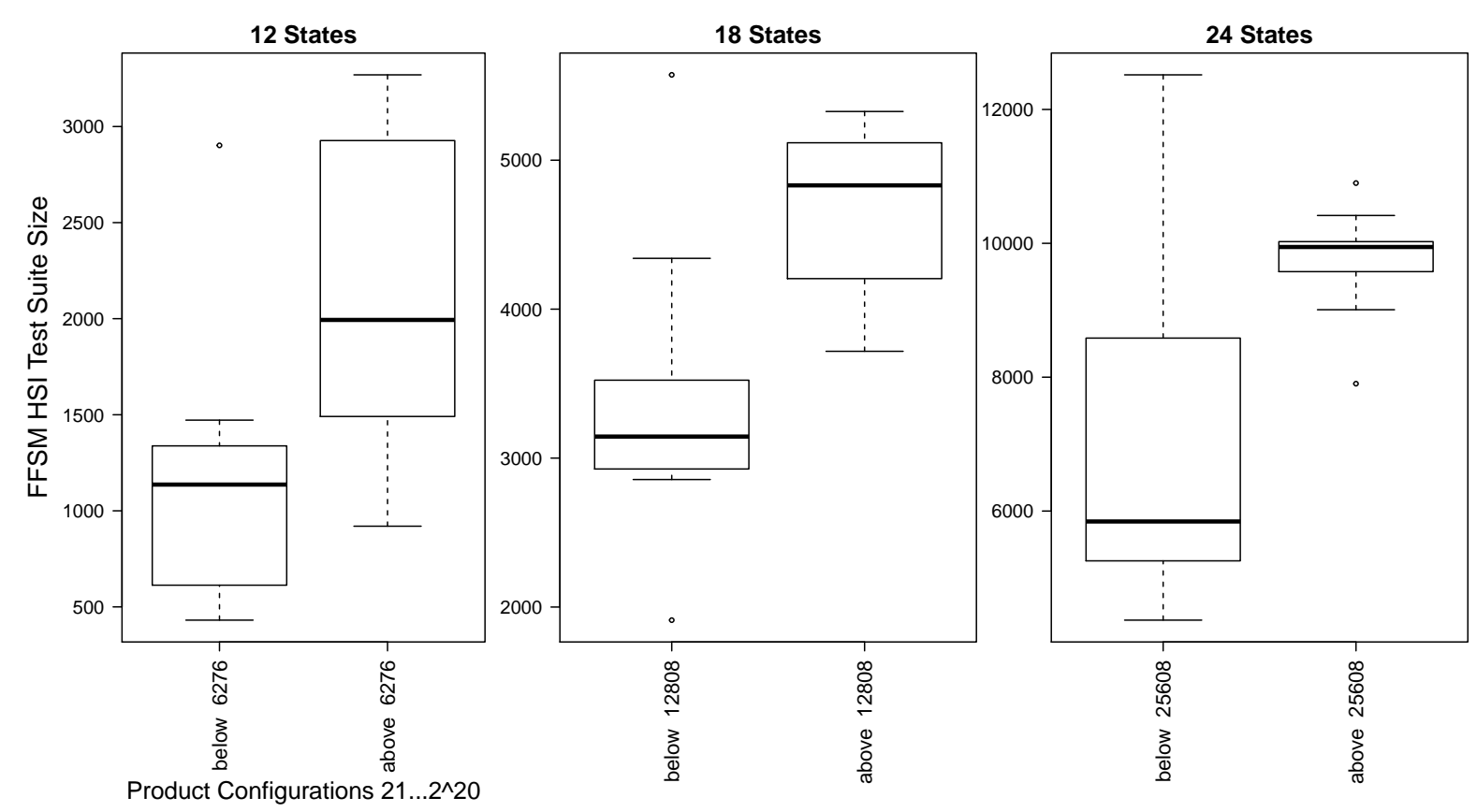

Figure 44 - Configurable test-suite size per kind of feature model.

About the number of conditional transitions, we noticed that FFSMs linked to feature models with more configurations than the median has fewer conditional transitions. This may be another coincidence or a consequence of the random generator. Thus, our experiment indicates a tendency to have fewer conditional transitions in cases where the feature model has fewer valid configurations. This indication may be a threat to validity as it may affect subsequent results.

\section{Q5- Is there a relation between the feature model and and the configurable test-suite size?}

Figure 44 shows the results for the relation between the configurable test-suite size and types of features models. The configurable test-suite is generated for each random FFSM using the extended HSI method presented in this thesis. For each random FFSM, we have a correspondent feature model. The results are generated for each FFSM group with 12, 18, and 24 states. In each group, we divide our 20 pairs of FFSMs and feature models in 2 subgroups of 10 each. The division was made using the median of the number of valid product configurations. The number of product configurations varies from 21 to $2^{20}$. Thus, in the first group of FFSMs with 12 conditional states the median of product configurations found from all feature models was 6276, then 12808 for the second group, and 25608 for the third group.

We noticed that the size of complete configurable test-suites is larger in those FFSMs which have feature models with more than the median of valid configurations in each group. This second result is a consequence of the first, where FFSMs with more than the median of valid products have fewer transitions (see Figure 43). When an FFSM has 

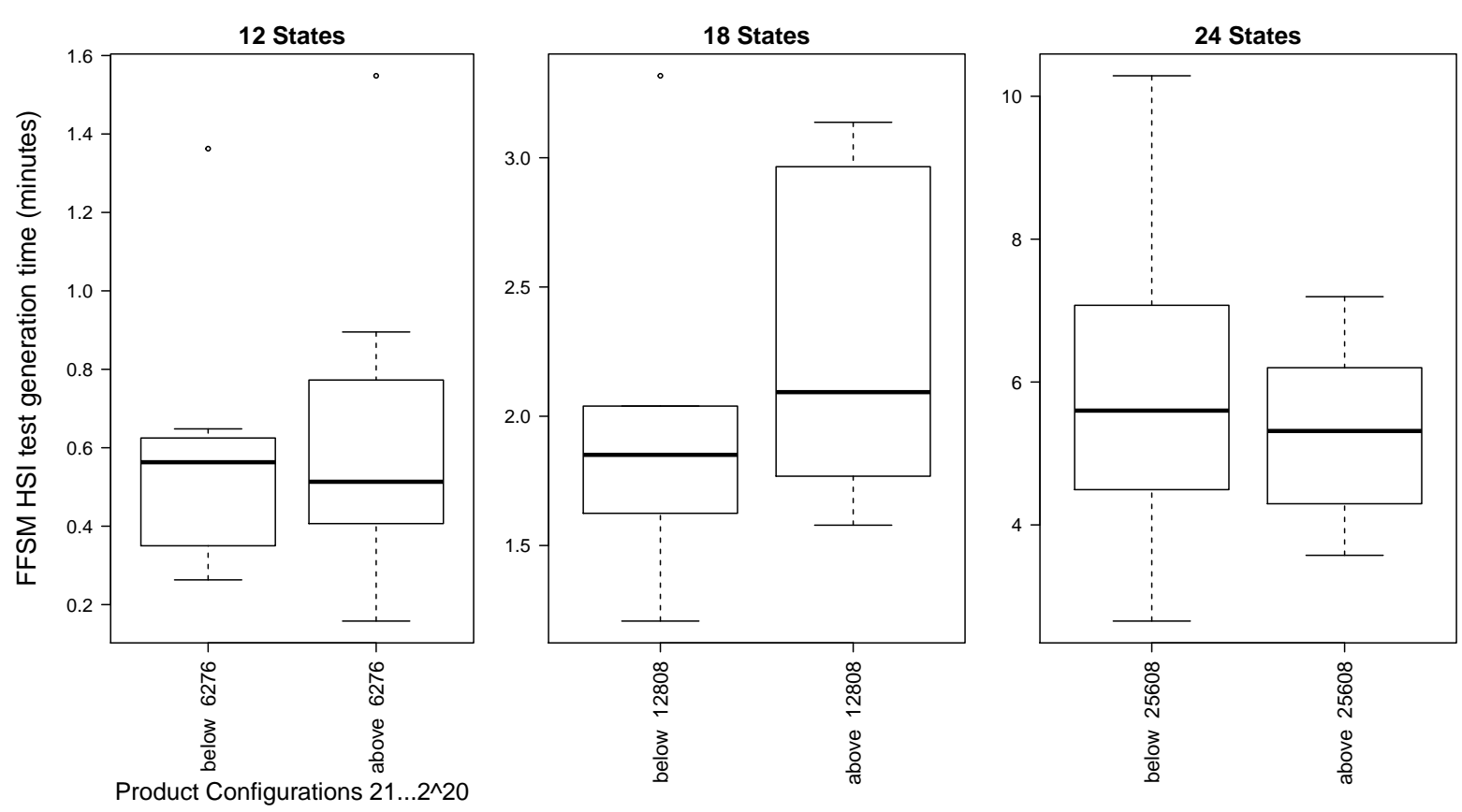

Figure 45 - Time required to execute the extended HSI method per kind of feature model.

fewer conditional transitions the algorithm has fewer options to find common conditional paths, resulting in more different conditional tests. Thus, our experiment indicates a tendency to have larger configurable test-suites for FFSMs when the feature model has many valid configurations but possibly affected by the number of fewer transitions. This indication may be a threat to validity as it may affect subsequent results.

Q6- Is there a relation between the feature model and the extended HSI test generation time?

Figure 45 shows the results for the relation between the time required to generate a configurable test-suite size and types of features models. The configurable test-suite is generated for each random FFSM using the extended HSI method presented in this thesis. For each random FFSM, we have a correspondent feature model. The results are generated for each FFSM group with 12, 18, and 24 states. In each group, we divide our 20 pairs of FFSMs and feature models in 2 subgroups of 10 each. The division was made using the median of the number of valid product configurations. The number of product configurations varies from 21 to $2^{20}$. Thus, in the first group of FFSMs with 12 conditional states the median of product configurations found from all feature models was 6276, then 12808 for the second group, and 25608 for the third group.

We noticed that the time required to generate configurable test-suites does not have a pattern from the 3 FFSM groups. Despite the larger configurable test-suites for those FFSMs with more than the median of valid configurations presented in Figure 44 the time required to generate them do not follow. Also, it does not have a direct relation to the FFSM size as presented in Figure 43. We believe that this is caused by the irregular 
number of required conditional paths and separating sequences. Thus, our experiment indicates no influence of the feature model for the time required to generate configurable test-suites.

\subsection{Body Comfort System Case Study}

We illustrate and evaluate our approach in a prototypical implementation using a case study from the automotive domain, a simplified Body Comfort System (BCS) for the VW Golf SPL (LITY et al., 2015). We use the BCS to reduce the threats to validity and contrast the results from randomly generated (F)FSMs with their real-world counterparts. The FeatureIDE tool (THÜM et al., 2014) was used for modeling feature models and their configurations. The original BCS system has 19 non-mandatory features and can have 11616 configurations. Moreover, BCS has several components running in parallel, and we found that flattening the whole system gives us a machine with more states than the maximal number of valid product configurations. Checking the initially connected property by finding a large number of paths is infeasible.

\subsubsection{Behavior Modeling}

Instead of composing the whole system we integrated few components. Figure 46 presents an adapted version of the feature model used to handle a part of the features with 4 non-mandatory features and 6 possible configurations for 4 components: Finger Protection ( $F P$ ) blocking the window movement when a finger is clamped in a window, Manual Power Window (ManPW) or alternatively Automatic Power Window (AutPW), and Central Locking System ( $C L S$ ) with optional Automatic Locking ( $A L$ ) when the car is driving.

The behavior of components can be checked individually or in groups. In groups, they can be composed of parallel regions using hierarchical models or expand them using flat models. Figure 47 presents the HFSM of four selected components of BCS. The original $150 \%$ behavioral model of each component can be found in (LITY et al., 2015). Inputs and outputs were abstracted from commands and guards and showed on the left.

Two alternative xor components were modeled in the PowerWindow region, and product specific transitions represent the behavior in each case. The only non-mandatory region is CentralLockingSystem which means that we are going to have to compose: (i) all three regions; and (ii) only the first two on the left. Region composition is explained in Figures 27 and 28. For the first composition the FingerProtection component has two states. Thus, the flat model may double, and the last component CentralLockingSystem also has two states which may double the number of states again. Figure 48 shows how we compose both combinations of regions and then connect to a single initial state. As 


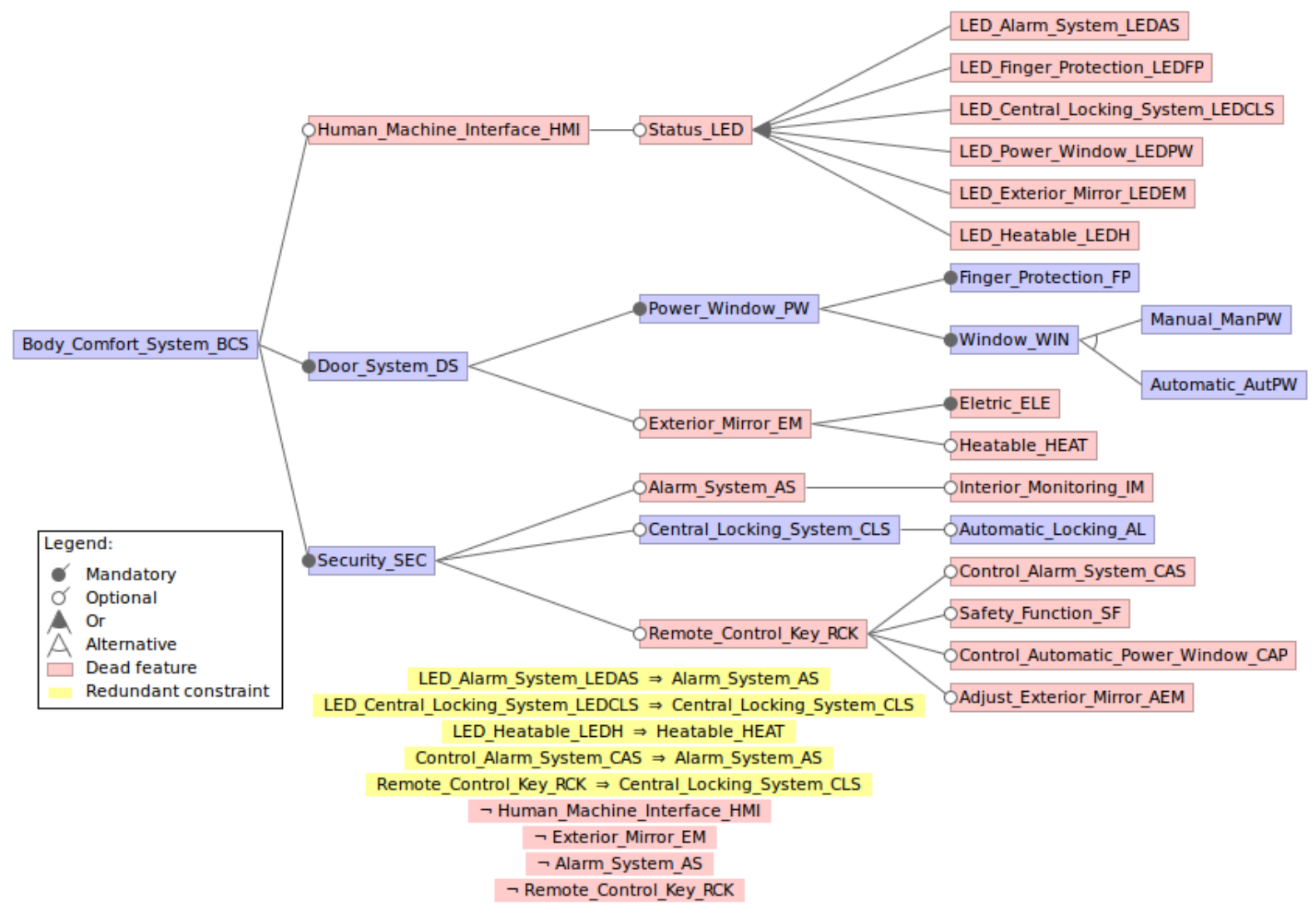

Figure 46 - Adapted Feature Model of the Body Comfort System (LITY et al., 2015).

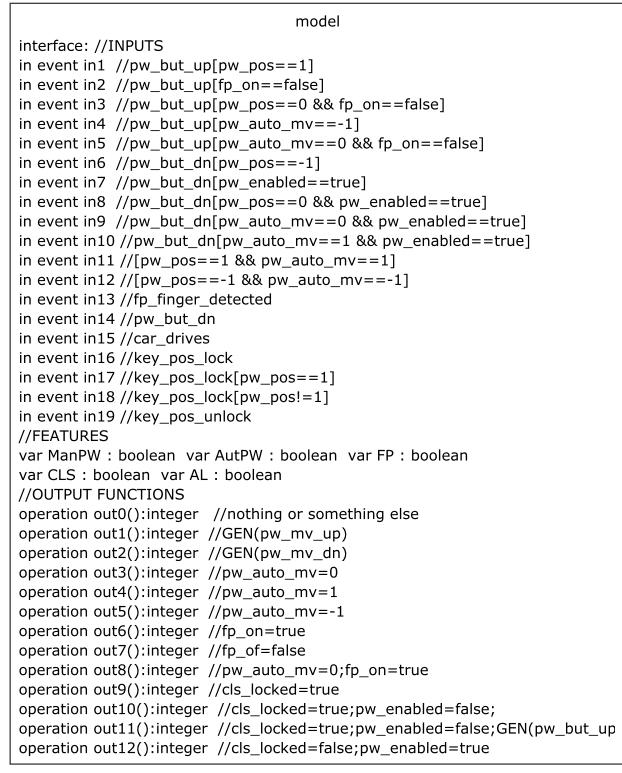

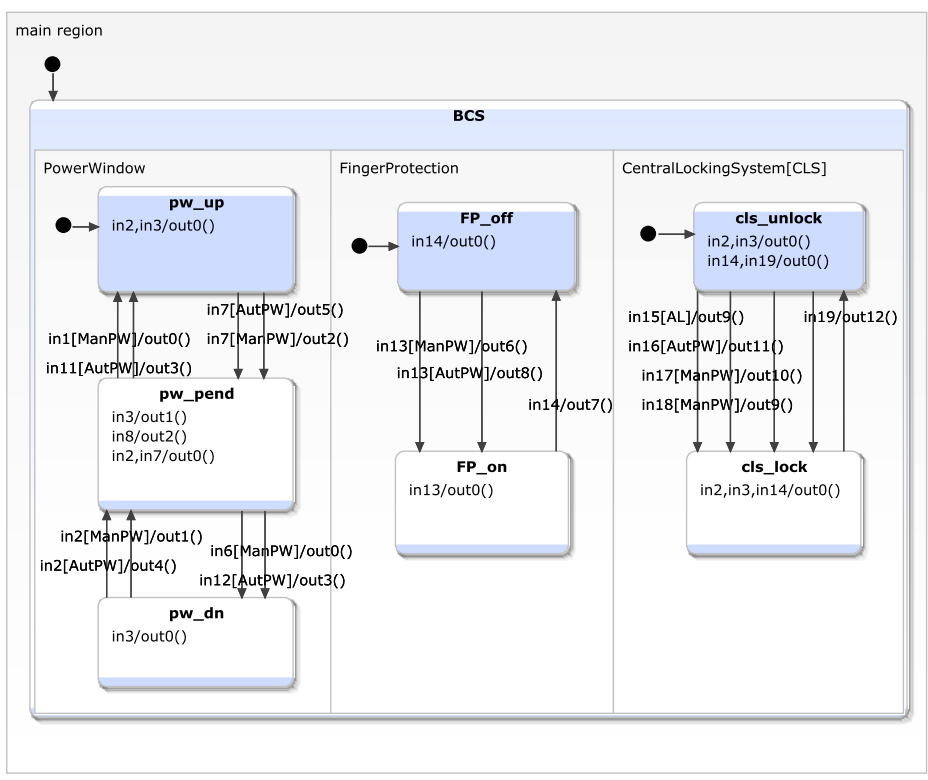

Figure 47 - HFSM of 4 composed components of BCS. 


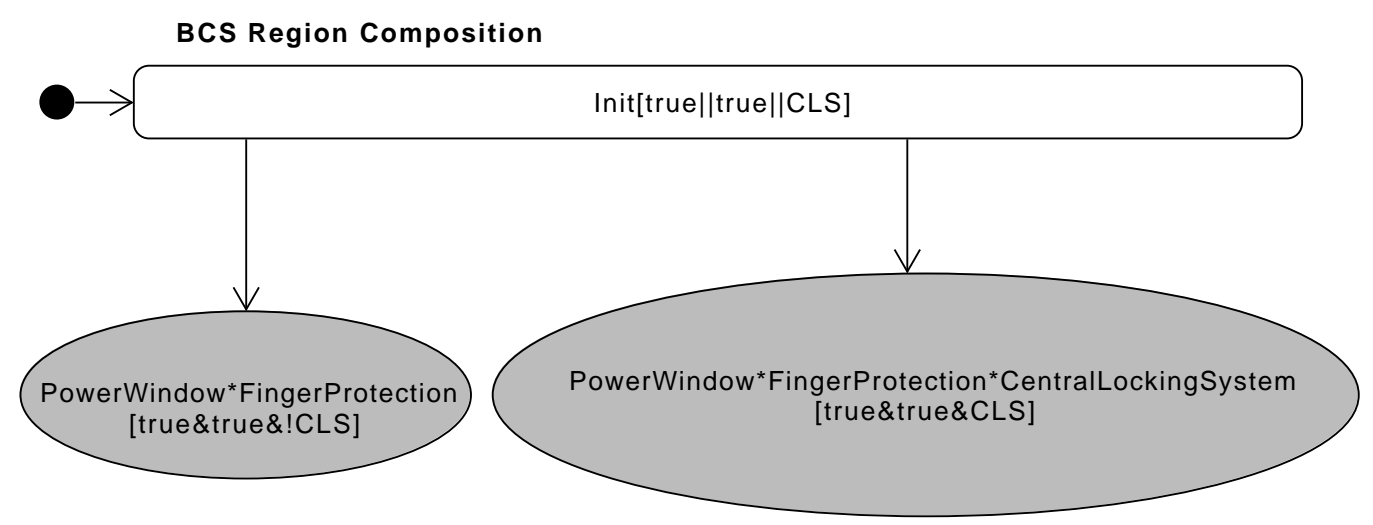

Figure 48 - Overview of the region composition of BCS HFSM.

stressed before, the initial state is the combination of the initial states from both region combinations which replace them and get their reaching and leaving transitions. This combination is needed as one region can be excluded, and then the target configuration of some transitions change.

The resulting semantic FFSM of BCS for the selected 4 components has 17 conditional states and 171 conditional transitions and it takes approximately 1 minute to perform all checks.

\subsubsection{Model Derivation}

Once the HFSM model is built and checked, a configuration can be chosen to derive other HFSMs. Figure 49 shows the selection of the Automatic Power Window component and leave the Central Locking System and Automatic Locking features unchecked which means that this configuration still has 3 out of 6 possible configurations.

The implemented tool has two types of model derivation: the first derivation ignores the open features and derives an HFSM for a subset of configurations. The resulting feature constraint for deriving the HFSM model is a conjunction of all marked (for inclusion or exclusion) features except those unmarked. The second derivation uses a feature constraint that is a conjunction of all marked features and those unmarked are considered as excluded which results in a feature constraint for a single product configuration. Figure 50 shows the first HFSM model derivation type for 3 product configurations. Figure 51 shows the second HFSM model derivation type for a single product configuration which excludes Central Locking System and Automatic Locking features. 


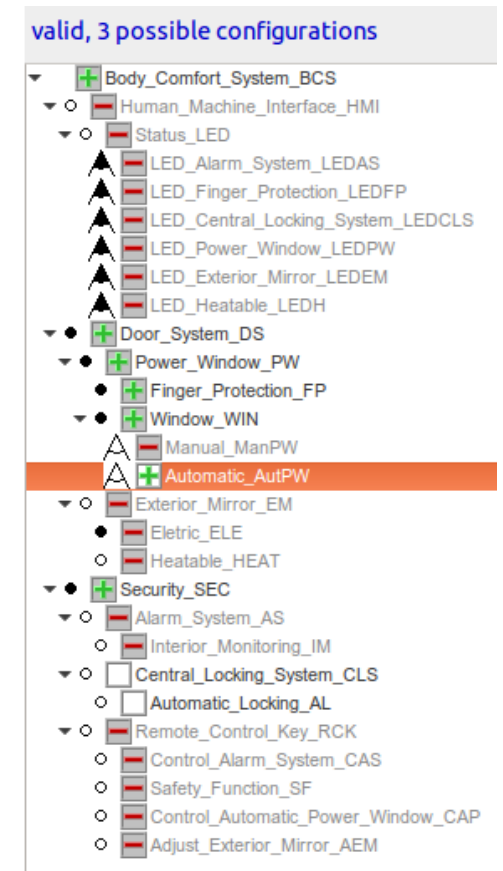

Figure 49 - Feature Model configuration selection for BCS.

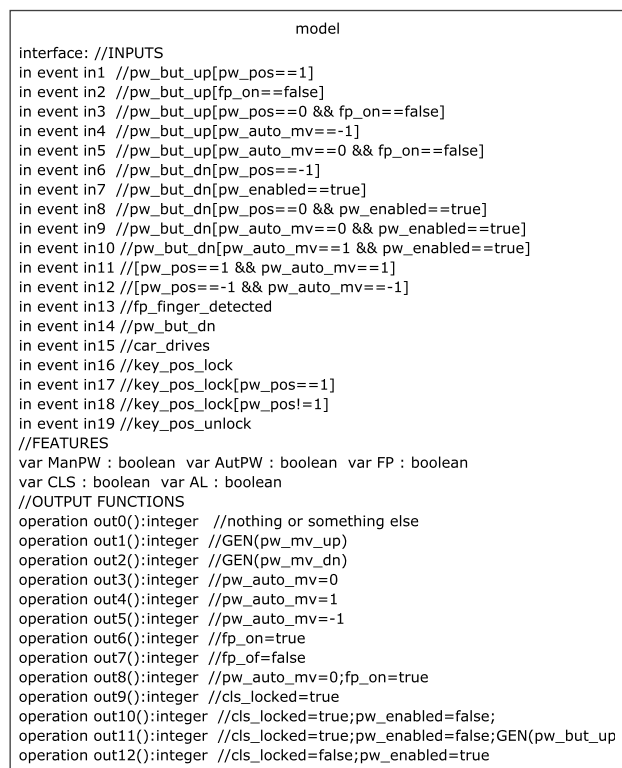

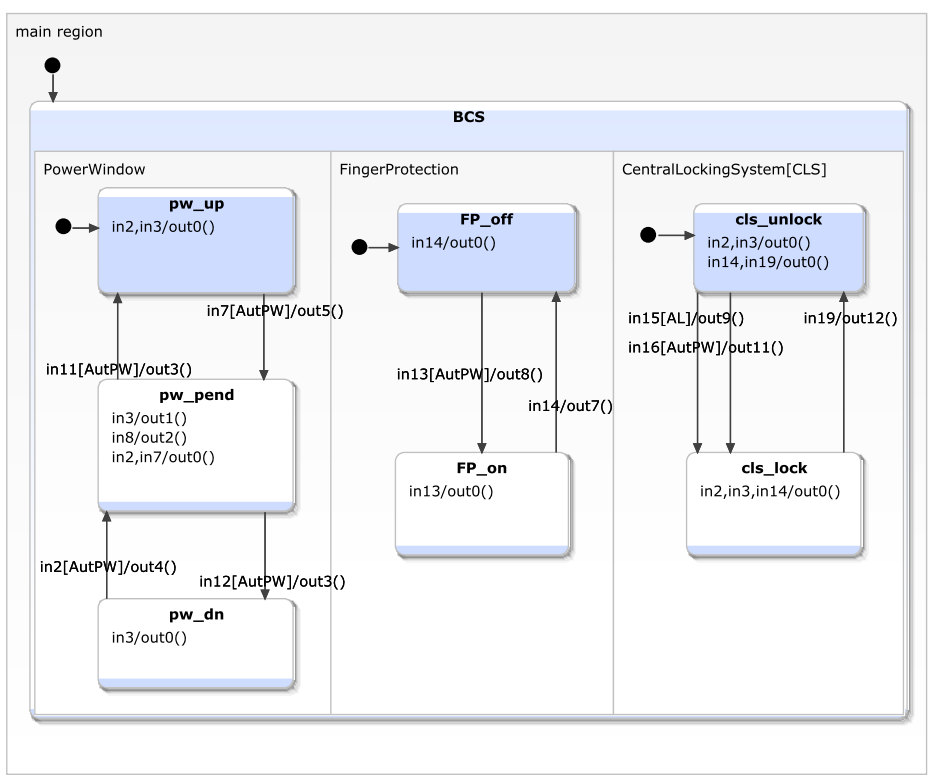

Figure 50 - HFSM derived for 3 configurations with 3 composed components. 

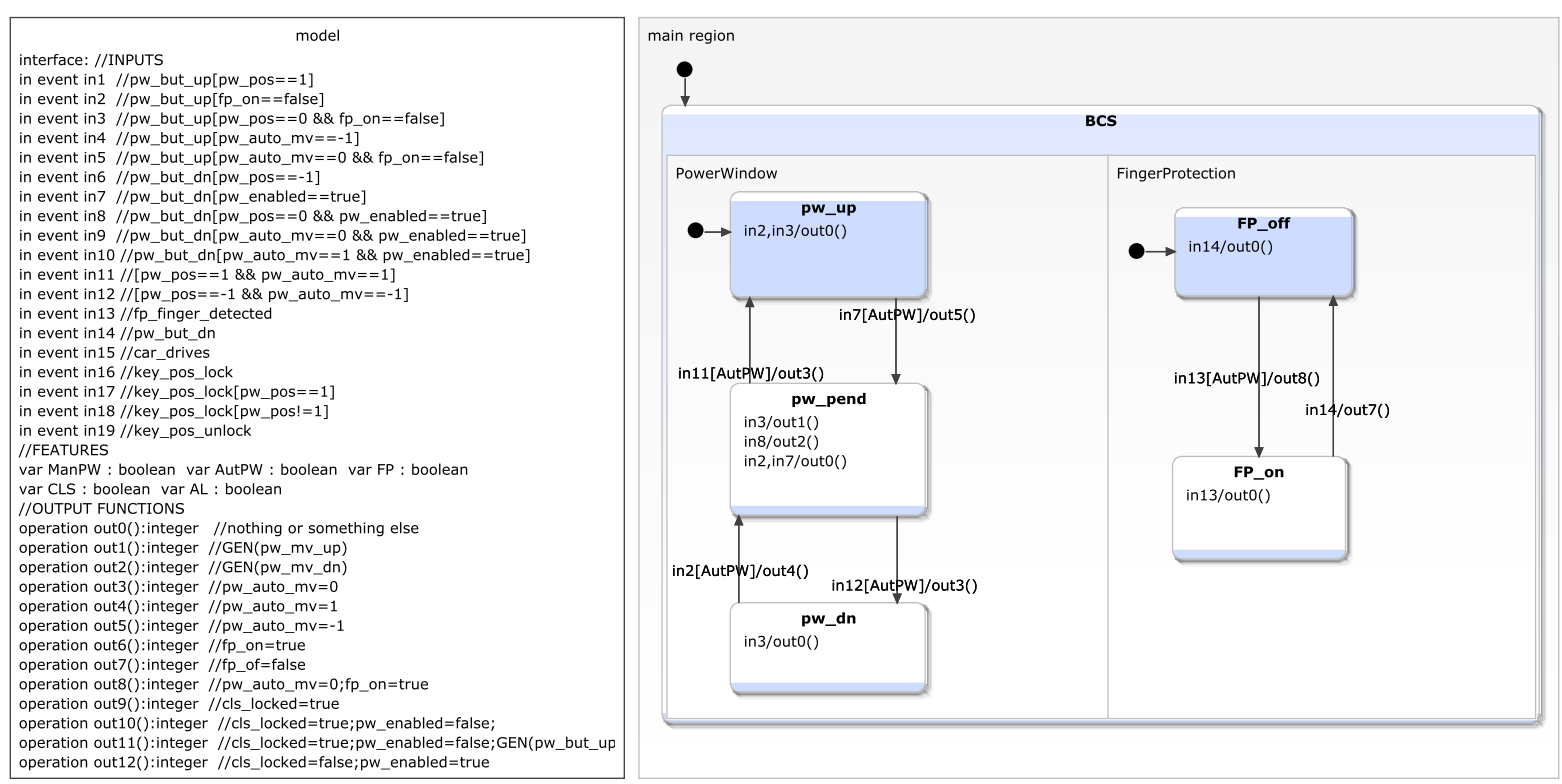

Figure 51 - HFSM derived for one configuration and 2 composed components.

\subsubsection{Test Generation}

Following our approach, the configurable test-suite obtained for the state coverage has 10 tests and size 38. The configurable test-suite obtained for the transition coverage has 148 tests and size 870. The configurable test-suite obtained for the full fault coverage has 527 tests and size 3585. The configurable test-suite generation takes approximately 1 minute.

Assuming that we have an FSM for each of the 6 valid configurations. Generating a complete test-suite using the HSI method for each FSM and calculating the number of new tests required we would need to concretize and execute 618 tests with an accumulated size of 4198. Thus, we conclude that our approach can automatically generate efficient, configurable test-suites for SPLs. Comparing our approach to the product-by-product approach for this case study our approach can save $15 \%$ of test effort based on the size of test-suites.

In section 7.4 we discuss examples of the redundancy problem in the SPL context.

\subsection{Test Redundancy Problem}

At this point two research directions were explored. In the first direction it was noticed in the experimental evaluation savings up to $36 \%$ of test effort regarding concretization and execution of test-cases for a set of 24 products using the proposed regression-based strategy. However, regarding exploiting regression-based techniques on SPLs there is a lack of a precise definition of similarity for generic coverage criteria. Moreover, we notice a drawback on our approach regarding the increasing size on the test-suite used as input to 
analyze and process which increases the algorithm execution time. Thus, we moved to the second direction of research.

In the second direction of research, we conducted an experimental study with random FFSMs and feature models to obtain the number of new test-cases required using the extended HSI method and the classic HSI. We compared the average number of testcases required for one FFSM that vary from 21 to $2^{20}$ SPL products to a set of 20 products with test-suites generated from HSI. The results indicate a reduction of approximately $50 \%$ of the number of new tests required for testing the entire SPL compared to only 20 products.

Regarding test design for the full fault coverage, it still requires the flattening of HFSMs into FFSMs. Another solution is slicing where parts of the system are selected to be tested. Moreover, we noticed that the reuse of test-cases between products is maximized (reducing redundancy) as long test-case generation methods are able to generate configurable test-suites. Figure 52 shows an example of the redundancy problem in SPL test design based on state machines. On the left we have a simple feature model and a state machine represented by an FFSM. Following the first research direction for productcentered test design (bottom) we have all four product configurations where the test goals are set based on the state coverage criteria. Common test design methods try to minimize the size of the final test-suite by selecting small input sequences. Note that for Product 1 and Product 2 the generation methods decide to use small but different input sequences for test goal TG2 (to reach state S2) due to the third test goal TG3 which using a different input sequence reduces the final test-suite size at that point for Product 2. Planning beforehand the minimal solution is a hard problem.

Following the second research direction for product line-centered test design we also have all four product configurations where the test goals are set based on the state coverage criteria. Here instead of generating test-cases for individual products, we consider the whole domain not seeing individual product configurations but sets of configurations. Thus, test goals are set for all product configurations where it is applicable. For example, to reach state 2 on TG2, a minimal set of test-cases is identified to be applied to every product configuration. As a result a configurable test-suite is created and instantiated according to a single or a set of product configurations. Note that the final accumulative test-suite of each direction is different as the first direction eventually requires more test-cases. The product-centered test design focus on the minimal test-suite for a single product configuration while on the product line-centered test design we minimize the overall number of test-cases which reduce test-case redundancy as shown in the experimental results. 


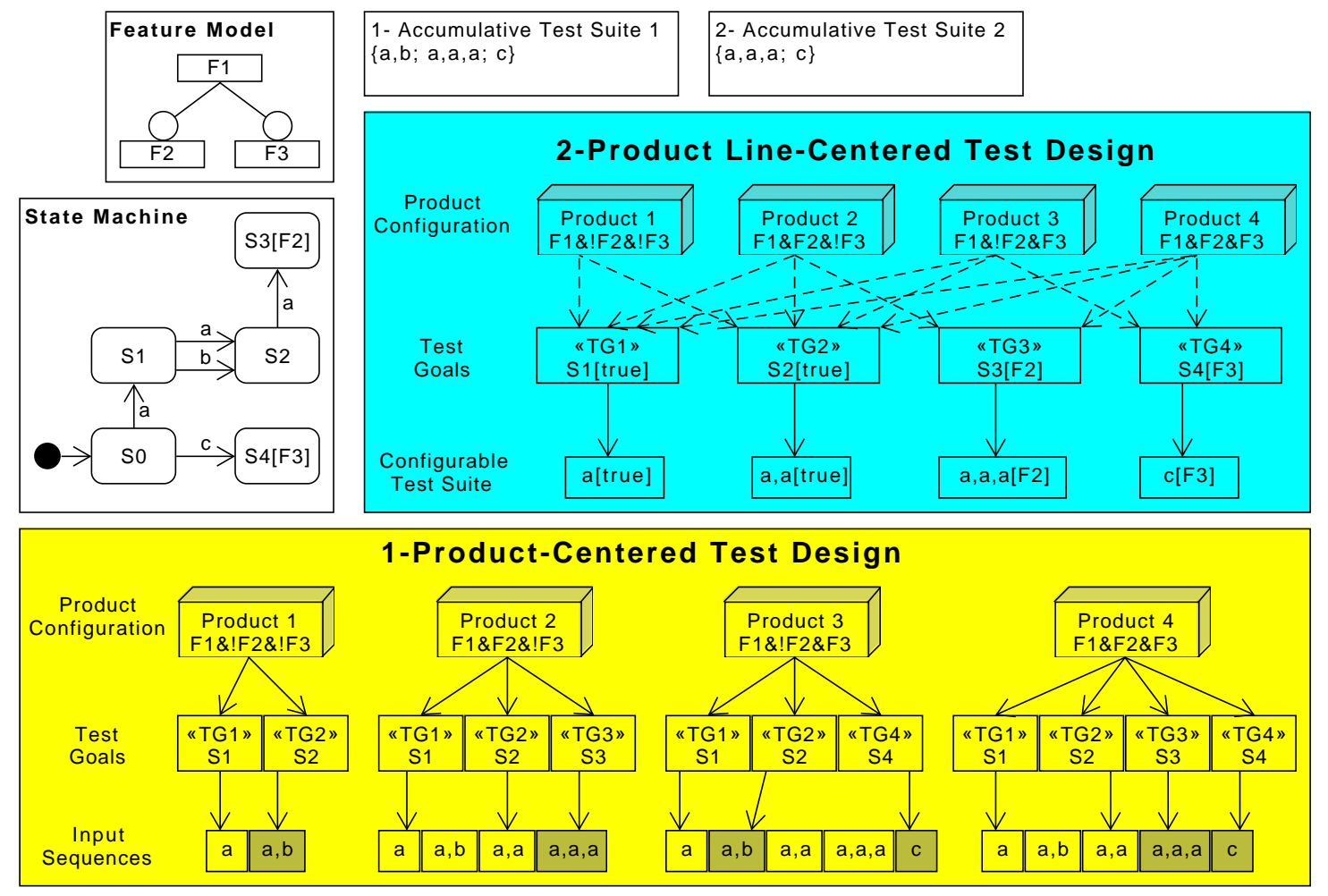

Figure 52 - Example of redundancy on SPL test design.

\subsection{Concluding Remarks}

In this chapter, we presented experimental results and a case study for both research directions explored in this thesis. For the product-centered test design direction, we noticed that up to $36 \%$ of test effort regarding concretization and execution of test-cases could be saved for a set of products using the proposed regression-based strategy. For the product line-centered test design direction we noticed a slightly (but not significant) increase in the number of new tests when compared individually, and a massive advantage (a reduction of approximately 50\%) when we need to test an SPL compared to 20 new products using the classic HSI method. Also, we investigate the applicability of our method by applying it to a realistic case study based on test design for FFSMs. 
CHAPTER

\section{8}

(1)

\section{CONCLUSIONS}

Software Product Lines (SPLs) (LINDEN; SCHMIF; ROMMES, 2007) provide an efficient mass customization approach at the cost of an initial extra effort to build the required architecture. As a consequence, testing the SPL becomes a challenging task due to the high number of different product configurations and other factors. One important factor is the test redundancy where several similar products share some components, and without a proper analysis of those products, part of test-suites are re-concretized and re-executed in vain.

One formalism used in the representation of the behavior of software systems is the classic Finite State Machine (FSM). There are several test-case generation techniques that were developed to generate test-suites for strong test criteria. A test-suite generated from strong test criteria once executed can detect several faults in the system compared to another test-suite from other test criteria. There is a hierarchy where one of the best is the full fault coverage for FSMs, and it was not yet fully extended to more expressive test models such as those based on labeled transition systems. Thus, in this thesis, one technique of the full fault coverage for FSMs is extended to the SPL context with the proposed FFSM.

The presented thesis is focused on model-based testing for SPLs. In detail, two test design approaches were proposed to derive test-cases avoiding redundancy and using strong coverage criteria. In our first test design approach, IRT-SPL can provide effort savings starting from $5 \%$ when we have more than 4 product configurations and the concretization value is 10, and goes up to $36 \%$ when we have 24 product configurations and the concretization value is 100 compared to other test reuse strategies.

In our second test design approach, we explore the variability to generate a small configurable test-suite for the entire SPL where most products reuse equivalent test-cases to avoid redundancy. We conducted an experimental study with random FFSMs and 
feature models, and for each feature model we selected 20 random products. We decided to randomize feature models and FFSMs to avoid bias of specific domains. A first test-suite was generated using the extended HSI method for an FFSM and compared to a second unified test-suite generated using the original HSI method for the selected 20 individual configurations. The results indicate a reduction in approximately $50 \%$ of the number of new tests required for testing using the first test-suite compared to the second. Also, the reduction percentage may decreases for less than 20 products and may increases for more than 20. We illustrate and evaluate our approach and tool by means of a case study from the automotive domain, using the Body Comfort System (BCS) for the VW Golf SPL (LITY et al., 2015). The results indicate a reduction in approximately $25 \%$ of the number of new tests required for testing a slice of the BCS for 6 products. Moreover, our examples indicates a reduction in approximately $35 \%$ of the number of new tests required for testing the AGM SPL for 6 products.

To conclude, we bring our research question again.

Is it possible to develop a test design method that provides low test redundancy and high fault detection for an $S P L$ ?

Yes, we can generate small test-suites for the full fault coverage (strong test criterion) in either test design approach developed in both research directions: the product-centered and the product line-centered. The drawback is that it requires the representation of the system(s) behavior using FSM-based specifications. In summary, according to our experimental results, our first test design approach favors the testing of few SPL products, while the second test design approach favors a large number of valid configurations that require testing.

The remainder of this chapter is organized as follows. Section 8.1 presents a summary of contributions. Section 8.2 discusses related works, while Section 8.3 presents related publications. Finally, Section 8.4 proposes future works of this thesis.

\subsection{Summary of Contributions}

This thesis focuses on a functional model-based testing approach for SPLs to reduce test-case redundancy using the full fault coverage in two directions. In the first direction, we explore a test-case reuse strategy named Incremental Regression-based Testing for Software Product Lines (IRT-SPL). The IRT-SPL can reduce test costs of a new derived product in the advanced development stages based on regression testing and the P method (SIMAO; PETRENKO, 2010). This research direction enables the efficient reuse of test-cases where only a few existing test-cases are selected and incremented to test a new derived product using fewer resources. The IRT-SPL strategy has three main contributions: 
- an incremental test-case reuse strategy;

- a test-case selection algorithm; and

- experimental evaluation using a case study of Mobile Media SPL.

In the second direction, we explore test design in the early development stages. Specifically, we propose a solution named Configurable Feature-based full Coverage testing of State Machines (CFC-SM). CFC-SM is a model-based test design approach that enables the automatic generation of reusable and customizable test artifacts for an SPL, which can be configured to test a set of products. The test engineer uses CFC-SM to create configurable feature-based state machines and generates test-cases for the full fault coverage criteria, specifically extending the HSI method (LUO et al., 1994). The CFC-SM approach has some contributions:

- proposing new configurable family-based test models for SPL;

- proposing family-based validation criteria for the full fault coverage criteria and proving them to coincide with their product-based counterparts;

- proposing the extension of the HSI test-case generation method and proving them to coincide with their product-based counterparts;

- implementing a model-based test generation tool with a graphical interface to support validation, derivation, and generation of family-based test artifacts.

- experimentally evaluating using random models and the automotive Body Comfort System SPL as a case study.

\subsection{Related Work}

Usually, an SPL can generate several similar products where only a few features vary from one to another. One challenge in SPL testing is the verification of products using a simplified behavioral model that take advantage of the similarity between products. There are proposals (CICHOS et al., 2011; CLASSEN et al., 2013) that provide a concise formalism for representing SPL behaviors in one model. However, they are focused on model checking (CLASSEN et al., 2013) or simple test criteria such as boundary tests (CICHOS et al., 2011). In this thesis, we take a step forward to extending the FSM-based formalism for SPLs looking forward to testing generation methods that use deterministic, initially connected and minimal machines to achieve better test-suites using the full fault coverage. 
Regarding regression-based approaches for SPLs, there are several incremental test approaches (EI-FAKIH; YEVTUSHENKO; BOCHMANN, 2004; PAP et al., 2007; UZUNCAOVA; KHURSHID; BATORY, 2010; CAPELLARI et al., 2012; BALLER; LOCHAU, 2014) devoted to generating, reusing, and optimizing test-suites for SPLs. El-Fakih et al. (EI-FAKIH; YEVTUSHENKO; BOCHMANN, 2004) adapted FSM-based test generation methods for conformance testing that allow the generation of test-cases only for the modified parts of an evolving specification. Pap et al. (PAP et al., 2007) extended their work and designed a bounded incremental algorithm that maintains two sets based on the HSI method (LUO et al., 1994). They utilize existing test-cases of the previous version of the system to generate test-cases for the modified version. Similarly, Capellari et al. (CAPELLARI et al., 2012) explored the FSM-based Testing of SPLs (FSM-TSPL) testing strategy where the $\mathrm{P}$ method is used to design new test-cases based on the last product derived. Uzuncaova et al. (UZUNCAOVA; KHURSHID; BATORY, 2010) also developed an incremental test generation approach that uses SAT-based analysis to develop tests suites for every product of an SPL, while Baller and Lochau (BALLER; LOCHAU, 2014) focused on test-suite optimization. Moreover, recent delta-oriented approaches (LOCHAU et al., 2012; LOCHAU et al., 2014; VARSHOSAZ; BEOHAR; MOUSAVI, 2015) developed regression-based SPL approaches to design and reuse test artifacts.

In contrast to current approaches, our work introduces a test reuse strategy focused on reducing test effort of a set of new SPL products. We analyze concretized test-cases of derived products to generate a small set of new test-cases to concretize for conformance testing. To our knowledge, there is no proposal that reuses test-cases from all previous products to reduce concretization effort for new SPL products using incremental test-case generation methods.

Regarding configurable test artifacts most modeling concepts for variability can be distinguished into three main approaches: annotative, compositional and transformational variability modeling (SCHAEFER et al., 2012). Compositional approaches for modeling variability capture variation by selecting specific component variants. Compositional variability modeling (HABER et al., 2011) allows a modular description of variability but limits the impact of changes to the applied composition technique. Transformational approaches represent variability by transformation of a base architectural model. Model transformation rules guide the derivation of products by performing additions, modifications or removals using variability. For example, Modeling delta (CLARKE; HELVENSTEIJN; SCHAEFER, 2011) can represent variability in the model transformation which a core system is developed, and subsequent products are derived by executing such transformations rules.

Annotative approaches use variant annotations (also called 150\%-models), e.g., UML stereotypes in UML models to define which model elements belong to specific 
product variants. In the orthogonal variability model (OVM) (POHL; METZGER, 2006), a separate variability representation with links to the architecture model replaces direct annotations. Some approaches (CZARNECKI; ANTKIEWICZ, 2005; GRÖNNINGER et al., 2008) propose a pruning-based approach to UML 150\% test model for SPLs, separating variability from the base models using mapping models.

Using an annotative $150 \%$ statechart test model and transition coverage criteria, Cichos et al.' s approach (CICHOS et al., 2011) presents SPL test design for complete test model coverage with subsequent product subset selection for test-suite execution. Weissleder et al. (WEISSLEDER; SOKENOU; SCHLINGLOFF, 2008) propose an approach for automatic test-suite derivation based on reusable UML state machine test models and OCL expressions. Similarly, Liu et al. (LIU; DEHLINGER; LUTZ, 2007) use Statecharts to model reusable components and instances can also be derived syntactically by pruning. As in Featured Transition Systems (CLASSEN et al., 2013), model fragments are annotated with presence conditions, i.e., Boolean expressions that define to which products a fragment belongs.

Model-based testing can be used in SPL testing. We refer the reader to Oster et al. (OSTER et al., 2012a) for a summary of model-based SPL testing approaches and to (BEOHAR; VARSHOSAZ; MOUSAVI, 2016; CZARNECKI et al., 2012; SCHAEFER et al., 2012; BENDUHN et al., 2015) for recent surveys and Thum et al.'s recent survey (THÜM et al., 2014) for a classification of different SPL analysis techniques. Some behavioral models proposed in the literature, e.g., these in (LIU; DEHLINGER; LUTZ, 2007; ASIRELLI et al., 2011; CLASSEN et al., 2011) are based on Finite State Machines or Labeled Transition Systems. They are mainly used to provide the formal specification of SPLs and their formal verification using model checking.

Our proposed approach for configurable test artifacts can be classified as a familybased and feature-oriented specification. To our knowledge, however, there only a few pieces of research that extend test models, test-case generation and test-case execution to the family-based level; examples of such work include earlier delta-oriented techniques such as (LOCHAU; KAMISCHKE, 2012; LOCHAU et al., 2014; VARSHOSAZ; BEOHAR; MOUSAVI, 2015) and feature-oriented approaches (BEOHAR; MOUSAVI, 2014b; DEVROEY et al., 2016). However, the approach proposed in (BEOHAR; MOUSAVI, 2014b; BEOHAR; MOUSAVI, 2014a) exploits a non-deterministic test-case generation algorithm (with no fault model or finite test-suite) and hence, semantic validation of test models is not an issue in their approach. Thus, we are not aware of any prior study one extending the FSM-based test-model validation and test-case generation techniques to the family-based setting that extends the full fault coverage. 


\subsection{Publications}

For this thesis, we have published two papers and two more were submitted to journals. The publications are:

1. Reducing the Concretization Effort in FSM-Based Testing of Software Product Lines (FRAGAL et al., 2017).

2. Validated Test Models for Software Product Lines: Featured Finite State Machines (FRAGAL; SIMAO; MOUSAVI, 2016).

3. Hierarchical Featured State Machines.

4. Extending HSI Test Generation Method for Software Product Lines.

\subsection{Future Work}

As future work, we plan to continue investigating a way to use HFSMs to generate test-cases without flattening the model for the full fault coverage criteria to handle scalability problems (state explosion). One major drawback of the proposed approach presented in this thesis is the scalability of the system to be tested. However, the scalability challenge exists for quite a while in computer science. In the case study it was noticed that the state explosion is higher than the product configuration explosion of the feature model. Test-case generation methods struggle to generate test-suites for strong test criteria in reasonable time and specifications with more than thousand states would take in a matter of several days to complete. Thus, the plan is to keep searching ways to solve the scalability problem or discover similar solutions that can be adapted.

Also, we plan to explore more aspects of configurable test artifacts for other test criteria and the use of feature constraints in other test models to also design configurable test models and test-cases. In this thesis, FSM-based test models were proposed, and the presented concepts could be extended to labeled transitions systems as they can represent different kinds of systems. 
AMMAnN, P.; OFfuTT, J. Introduction to Software Testing. [S.l.]: Cambridge University, 2008. 344 p. Citations on pages 25, 27, 28, 29, 31 e 34.

ASIRELLI, P.; BEEK, M. H. ter; GNESI, S.; FANTECHI, A. Formal Description of Variability in Product Families. In: 15th International Software Product Line Conference (SPLC). IEEE, 2011. p. 130-139. ISBN 978-1-4577-1029-2. Available: $<$ http://ieeexplore.ieee.org/lpdocs/epic03/wrapper.htm?arnumber $=6030054>$. Citation on page 137.

BALLER, H.; LOCHAU, M. Towards Incremental Test Suite Optimization for Software Product Lines. In: 6th International Workshop on Feature-Oriented Software Development (FOSD). [S.1.]: ACM, 2014. p. 30-36. Citation on page 136.

BASIN, D.; DOSER, J.; LODDERSTEDT, T. Model driven security. ACM Transactions on Software Engineering and Methodology, ACM, v. 15, n. 1, p. 39-91, 2006. ISSN 1049331X. Available: <http://portal.acm.org/citation.cfm?doid=1125808.1125810>. Citation on page 35 .

BATORY, D. Feature Models, Grammars, and Propositional Formulas. In: 9th international conference on Software Product Lines (SPLC). Springer, 2005. p. 7-20. Available: <http://link.springer.com/10.1007/11554844_3>. Citation on page 49.

BENDUHN, F.; THÜM, T.; LOCHAU, M.; LEICH, T.; SAAKE, G. A Survey on Modeling Techniques for Formal Behavioral Verification of Software Product Lines. In: 9th International Workshop on Variability Modelling of Software-intensive Systems (VaMoS). ACM, 2015. p. 80-87. ISBN 978-1-4503-3273-6. Available: <http: //dl.acm.org/citation.cfm?id=2701319>. Citation on page 137 .

BEOHAR, H.; MOUSAVI, M. Spinal Test Suites for Software Product Lines. In: 9th Workshop on Model-Based Testing (MBT). ACM, 2014. (Eletronic Proceedings in Theoretical Computer Science (EPTCS), v. 141), p. 44-55. Available: <https://doi.org/10. 4204/EPTCS.141>. Citations on pages 72 e 137.

BEOHAR, H.; MOUSAVI, M. R. Input-output Conformance Testing Based on Featured Transition Systems. In: 29th Annual ACM Symposium on Applied Computing (SAC). ACM, 2014. p. 1272-1278. ISBN 978-1-4503-2469-4. Available: <http://doi.acm. org/10.1145/2554850.2554949>. Citations on pages 72 e 137.

BEOHAR, H.; VARSHOSAZ, M.; MOUSAVI, M. R. Basic behavioral models for software product lines: Expressiveness and testing pre-orders. Science of Computer Programming, Elsevier, v. 123, p. 42-60, 2016. Citation on page 137.

BLECH, J. O.; MOU, D.; RATIU, D. Reusing Test-Cases on Different Levels of Abstraction in a Model Based Development Tool. Electronic Proceedings in Theoretical Computer Science, v. 80, n. Mbt, p. 13-27, feb 2012. ISSN 2075-2180. Available: $<$ http://arxiv.org/abs/1202.6119v1>. Citation on page 39. 
BRIAND, L. C.; LABICHE, Y.; HE, S. Automating regression test selection based on UML designs. Information and Software Technology, v. 51, n. 1, p. 16-30, Jan. 2009. ISSN 09505849. Available: <http://linkinghub.elsevier.com/retrieve/pii/S095058490800133X>. Citation on page 30.

BROY, M.; JONSSON, B.; KATOEN, J.; LEUCKER, M.; PRETSCHNER, A. ModelBased Testing of Reactive Systems: Advanced Lectures. [S.l.]: Springer, 2005. 1-659 p. ISBN 3540262784. Citations on pages 35 e 40.

CAPELlARI, M. L.; GIMENES, I. M. S.; SIMAO, A.; ENDO, A. T. Towards Incremental FSM-based Testing of Software Product Lines. In: 11th Brazilian Symposium of Software Quality (SBQS). SBC, 2012. p. 9-23. Available: <http://www.sbqs2012.com. $\mathrm{br} />$. Citations on pages 60, 113 e 136 .

CHEN, Y.; PROBERT, R. L.; URAL, H. Regression test suite reduction based on SDL models of system requirements. Journal of Software Maintenance and Evolution: Research and Practice, John Wiley and Sons, v. 21, n. 6, p. 379-405, 2009. ISSN 1532-0618. Available: <http://dx.doi.org/10.1002/smr.415>. Citation on page 113.

CHOW, T. Testing Software Design Modeled by Finite-State Machines. IEEE Transactions on Software Engineering, SE-4, n. 3, p. 178-187, 1978. ISSN 0098-5589. Available: $<$ http://ieeexplore.ieee.org/lpdocs/epic03/wrapper.htm?arnumber=1702519>. Citations on pages 45 e 116 .

CICHOS, H.; OSTER, S.; LOCHAU, M.; SCHüRR, A. Model-Based Coverage-Driven Test Suite Generation for Software Product Lines. In: 14th International Conference on Model Driven Engineering Languages and Systems (MODELS). Springer, 2011. p. 425-439. ISBN 978-3-642-24484-1. Available: <http://dl.acm.org/citation.cfm? $\mathrm{id}=2050655.2050698>$. Citations on pages 20, 21, 135 e 137.

CLARKE, D.; HELVENSTEIJN, M.; SCHAEFER, I. Abstract delta modeling. In: 9th International Conference on Generative Programming and Component Engineering (GPCE). [S.l.]: ACM, 2011. v. 46, n. 2, p. 13-22. Citation on page 136.

CLASSEN, A.; CORDY, M.; SCHOBBENS, P.-Y.; HEYMANS, P.; LEGAY, A.; RASKIN, J.-F. Featured Transition Systems: Foundations for Verifying Variability-Intensive Systems and Their Application to LTL Model Checking. IEEE Transactions on Software Engineering, IEEE, v. 39, n. 8, p. 1069-1089, 2013. ISSN 0098-5589. Available: <http: //ieeexplore.ieee.org/lpdocs/epic03/wrapper.htm?arnumber $=6389685>$. Citations on pages 21, 39, 135 e 137 .

CLASSEN, A.; HEYMANS, P.; SCHOBBENS, P.-Y.; LEGAY, A. Symbolic model checking of software product lines. In: 33rd International Conference on Software Engineering (ICSE). ACM, 2011. p. 321-330. ISBN 9781450304450. Available: $<$ http://portal.acm.org/citation.cfm?doid $=1985793.1985838>$. Citations on pages 39 e 137.

CLEMENTS, P.; NORTHROP, L. Software Product Lines: Practices and Patterns. [S.l.]: Addison-Wesley Longman Publishing Co., Inc., 2001. 608 p. Citation on page 46.

CZARNECKI, K.; ANTKIEWICZ, M. Mapping Features to Models: A Template Approach Based on Superimposed Variants. In: 4th International Conference on Generative 
Programming and Component Engineering (GPCE). Springer, 2005. p. 422-437. Available: <http://link.springer.com/10.1007/11561347_28>. Citation on page 137.

CZARNECKI, K.; GRÜNBACHER, P.; RABISER, R.; SCHMID, K.; WASOWSKI, A. Cool features and tough decisions. In: 6th International Workshop on Variability Modeling of Software-Intensive Systems (VaMoS). ACM, 2012. p. 173-182. ISBN 9781450310581. Available: <http://dl.acm.org/citation.cfm?doid=2110147.2110167>. Citation on page 137.

CZARNECKI, K.; PIETROSZEK, K. Verifying feature-based model templates against wellformedness OCL constraints. In: Proceedings of the 5th international conference on Generative programming and component engineering (GPCE). New York, New York, USA: ACM Press, 2006. p. 211-220. ISBN 1595932372. Available: <http: //portal.acm.org/citation.cfm?doid $=1173706.1173738>$. Citation on page 38 .

DALAL, S. R.; JAIN, A.; KARUNANITHI, N.; LEATON, J. M.; LOTT, C. M.; PATTON, G. C.; HOROWITZ, B. M. Model-based testing in practice. In: 21st international conference on Software engineering - ICSE 99. ACM, 1999. p. 285-294. ISBN 1581130740. Available: <http://portal.acm.org/citation.cfm?doid=302405.302640>. Citation on page 27.

DEV, R.; JÄÄSKELÄINEN, A.; M., K. Advances in Computers. [S.l.]: Elsevier, 2012. 395 p. Citation on page 38.

DEVROEY, X.; PERROUIN, G.; PAPADAKIS, M.; LEGAY, A.; SCHOBBENS, P.Y.; HEYMANS, P. Featured model-based mutation analysis. In: 38th International Conference on Software Engineering (ICSE). ACM, 2016. p. 655-666. ISBN 9781-4503-3900-1. Available: <http://doi.acm.org/10.1145/2884781>. Citation on page 137.

DONG, Y.; WANG, Y.; LAU, M. F.; LIN, S. Y. Experiments on test case reuse of test coverage criteria. In: Proceedings - Symposia and Workshops on Ubiquitous, Autonomic and Trusted Computing in Conjunction with the UIC 2010 and ATC 2010 Conferences, UIC-ATC 2010. IEEE, 2010. p. 277-281. ISBN 978-1-42449043-1. Available: <http://ieeexplore.ieee.org/lpdocs/epic03/wrapper.htm?arnumber= $5667187>$. Citation on page 31.

EI-FAKIH, K.; YEVTUSHENKO, N.; BOCHMANN, G. FSM-based incremental conformance testing methods. IEEE Transactions on Software Engineering, IEEE, v. 30, n. 7, p. 425-436, 2004. ISSN 0098-5589. Citations on pages 113, 117 e 136.

ENDO, A. T.; SIMAO, A. Evaluating test suite characteristics, cost, and effectiveness of FSM-based testing methods. Information and Software Technology, Elsevier, v. 55, n. 6, p. 1045-1062, 2013. ISSN 09505849. Available: <http://www.sciencedirect.com/ science/article/pii/S0950584913000128> . Citation on page 45.

ENGSTRÖM, E.; RUNESON, P. Decision Support for Test Management and Scope Selection in a Software Product Line Context. In: 4th International Conference on Software Testing, Verification and Validation Workshops (ICST). IEEE, 2011. p. 262-265. ISBN 978-1-4577-0019-4. Available: <http://ieeexplore.ieee.org/lpdocs/epic03/ wrapper.htm?arnumber $=5954417>$. Citation on page 55 . 
ENGSTROM, E.; RUNESON, P. Test overlay in an emerging software product line - An industrial case study. Information and Software Technology, Elsevier, v. 55, n. 3, p. 581-594, 2013. ISSN 09505849. Available: < http://linkinghub.elsevier.com/retrieve/pii/ S0950584912001061>. Citations on pages 11, 20, 52 e 53.

FIGUEIREDO, E.; CACHO, N.; SANT'ANNA, C.; MONTEIRO, M.; KULESZA, U.; GARCIA, a.; SOARES, S.; FERRARI, F.; KHAN, S.; FILHO, F.; DANTAS, F. Evolving software product lines with aspects. 30th International Conference on Software Engineering, ACM, p. 261-270, 2008. ISSN 0270-5257. Available: <http://portal.acm. org/citation.cfm?doid $=1368088.1368124>$. Citation on page 114 .

FISCHBEIN, D.; UCHITEL, S.; BRABERMAN, V. A foundation for behavioural conformance in software product line architectures. In: Proceedings of the ISSTA 2006 workshop on Role of software architecture for testing and analysis (ROSATEA). New York, New York, USA: ACM, 2006. p. 39-48. ISBN 1595934596. Available: <http://portal.acm.org/citation.cfm?doid=1147249.1147254>. Citation on page 21 .

FRAGAL, V. H.; SIMAO, A.; ENDO, A. T.; MOUSAVI, M. R. Reducing the Concretization Effort in FSM-Based Testing of Software Product Lines. In: Software Testing, Verification and Validation Workshops (ICSTW). [S.1.]: IEEE, 2017. p. 329-336. Citation on page 138.

FRAGAL, V. H.; SIMAO, A.; MOUSAVI, M. R. Validated Test Models for Software Product Lines: Featured Finite State Machines. In: 13th International Conference on Formal Aspects of Component Software (FACS). [S.l.]: Springer, 2016. p. 210-227. Citations on pages 69 e 138.

FRASER, G.; WOTAWA, F.; AMMANN, P. E. Testing with model checkers: a survey. Software Testing, Verification and Reliability, John Wiley and Sons, v. 19, n. 3, p. 215-261, 2009. ISSN 09600833. Available: <http://doi.wiley.com/10.1002/stvr.402>. Citation on page 39.

GAMMA, E.; BECK, K. JUnit 4.1 - A Testing Framework for Java. 2006. Citation on page 35 .

Graham D., F. M. Experiences of Test Automation: Case Studies of Software Test Automation. [S.l.]: Addison-Wesley Professional, 2012. 672 p. Citations on pages 38,60 e 115.

GREENFIELD, J.; SHORT, K. Software factories: Assembling applications with patterns, models, frameworks and tools. In: 18th Annual ACM SIGPLAN Conference on Object-oriented Programming, Systems, Languages, and Applications. ACM, 2003. (OOPSLA '03), p. 16-27. ISBN 1-58113-751-6. Available: <http://doi.acm.org/10. 1145/949344.949348>. Citation on page 19.

GRÖNNINGER, H.; KRAHN, H.; PINKERNELL, C.; RUMPE, B. Modeling Variants of Automotive Systems using Views. In: Tagungsband Modellierungs-Workshop MBEFF: Modellbasierte Entwicklung von eingebetteten Fahrzeugfunktionen. [S.1.]: TU Braunschweig, 2008. p. 1-14. Citations on pages 21 e 137. 
HABER, A.; RENDEL, H.; RUMPE, B.; SCHAEFER, I.; LINDEN, F. van der. Hierarchical Variability Modeling for Software Architectures. In: 15th International Software Product Line Conference (SPLC). IEEE, 2011. p. 150-159. ISBN 978-1-4577-1029-2. Available: <http://ieeexplore.ieee.org/document/6030056/>. Citation on page 136.

HAREL, D.; NAAMAD, A. The STATEMATE semantics of statecharts. ACM Transactions on Software Engineering and Methodology (TOSEM), v. 5, n. 4, p. 293-333, 1996. Citations on pages 89, 90, 93 e 96.

HEIDER, W.; RABISER, R.; GRUNBACHER, P.; LETTNER, D. Using regression testing to analyze the impact of changes to variability models on products. In: 16th International Software Product Line Conference (SPLC). ACM Press, 2012. v. 1, p. 196-205. ISBN 9781450310949. Available: <http://dl.acm.org/citation.cfm?doid=2362536. $2362563>$. Citation on page 55 .

HOWDEN, W. E. Software Engineering and Technology: Functional Program Testing and Analysis. [S.l.]: McGrall-Hill, 1987. 175 p. Citation on page 32.

IEEE. 610.12-1990 - IEEE Standard Glossary of Software Engineering Terminology. 1990. 1-84 p. Citation on page 26.

IT-BEAUFTRAGTEN, R. der. V-Modell. 1997. Citations on pages 11, 27 e 28.

JINHUA, L.; QIONG, L.; JING, L. The W-Model for Testing Software Product Lines. In: International Symposium on Computer Science and Computational Technology. IEEE, 2008. p. 690-693. ISBN 978-0-7695-3498-5. Available: <http://ieeexplore.ieee. org/lpdocs/epic03/wrapper.htm?arnumber $=4731520>$. Citations on pages 11, 51 e 52 .

KAMISCHKE, J.; LOCHAU, M.; BALLER, H. Conditioned model slicing of featureannotated state machines. In: Proceedings of the 4th International Workshop on Feature-Oriented Software Development - FOSD '12. ACM Press, 2012. p. 9-16. ISBN 9781450313094. Available: <http://dl.acm.org/citation.cfm?doid=2377816. $2377818>$. Citations on pages 21 e 39.

KANG, K. C.; COHEN, S. G.; HESS, J. A.; NOVAK, W. E.; PETERSON, A. S. FeatureOriented Domain Analysis (FODA) Feasibility Study. [S.l.], 1990. 1-161 p. Citation on page 48.

KANG, S.; LEE, J.; KIM, M.; LEE, W. Towards a Formal Framework for Product Line Test Development. In: 7th IEEE International Conference on Computer and Information Technology (CIT). IEEE, 2007. p. 921-926. ISBN 0-7695-2983-6. Available: <http://ieeexplore.ieee.org/lpdocs/epic03/wrapper.htm?arnumber $=4385203>$. Citation on page 48 .

KIM, K.; KIM, H.; AHN, M.; SEO, M.; CHANG, Y.; KANG, K. C. ASADAL: a tool system for co-development of software and test environment based on product line engineering. In: 28th international conference on Software engineering (ICSE). ACM, 2006. p. 783-786. ISBN 1595933751. Available: < http://portal.acm.org/citation.cfm?doid=1134285. 1134412>. Citation on page 55.

LASALlE, J.; PEUREUX, F.; GUILlET, J. Automatic test concretization to supply end-to-end MBT for automotive mechatronic systems. In: Proceedings of the First International Workshop on End-to-End Test Script Engineering - ETSE '11. 
ACM Press, 2011. p. 16-23. ISBN 9781450308083. Available: <http://www.scopus.com/ inward $/$ record.url? eid $=2-\mathrm{s} 2.0-80052180430\{\&\}$ partnerID $=$ tZOtx3y1 $>$. Citation on page 20.

LINDEN, F.; SCHMIF, K.; ROMMES, E. Software Product Lines in Action. [S.1.]: Springer, 2007. 1-353 p. Citations on pages 19, 46, 47, 49, 51 e 133.

LINZHANG, W.; JIESONG, Y.; XIAOFENG, Y.; JUN, H.; XUANDONG, L.; GUOLIANG, Z. Generating Test Cases from UML Activity Diagram based on Gray-Box Method. In: 11th Asia-Pacific Software Engineering Conference. IEEE, 2004. p. 284-291. ISBN 0-7695-2245-9. Available: <http://ieeexplore.ieee.org/lpdocs/epic03/wrapper.htm? arnumber $=1371930>$. Citation on page 32 .

LITY, S.; LACHMANN, R.; LOCHAU, M.; SCHAEFER, I. Delta-oriented Software Product Line Test Models - The Body Comfort System Case Study. TU Braunschweig, 2015. 302 p. Citations on pages 12, 126, 127 e 134.

LITY, S.; LOCHAU, M.; SCHAEFER, I.; GOLTZ, U. Delta-oriented model-based SPL regression testing. In: 3rd International Workshop on Product LinE Approaches in Software Engineering (PLEASE). IEEE, 2012. p. 53-56. ISBN 978-1-4673-1751-1. Available: <http://ieeexplore.ieee.org/lpdocs/epic03/wrapper.htm?arnumber $=6229772>$. Citation on page 54 .

LIU, J.; DEHLINGER, J.; LUTZ, R. Safety analysis of software product lines using state-based modeling. Journal of Systems and Software, Elsevier, v. 80, n. 11, p. 1879-1892, 2007. ISSN 01641212. Available: <http://linkinghub.elsevier.com/retrieve/pii/ S016412120700057X $>$. Citation on page 137.

LLANA, A. G. Model Driven Software Product Line Engineering: System Variability View and Process. 520 p. Phd Thesis (PhD Thesis), 2012. Citation on page 36 .

LOCHAU, M.; KAMISCHKE, J. Parameterized Preorder Relations for Model-Based Testing of Software Product Lines. In: 5th International Symposium on Leveraging Applications of Formal Methods, Verification and Validation. Technologies for Mastering Change (ISoLA). Springer, 2012. p. 223-237. Available: < http://link. springer.com/10.1007/978-3-642-34026-0_17>. Citations on pages 55 e 137.

LOCHAU, M.; LITY, S.; LACHMANN, R.; SCHAEFER, I.; GOLTZ, U. Delta-oriented model-based integration testing of large-scale systems. Journal of Systems and Software, Elsevier, v. 91, p. 63-84, 2014. ISSN 01641212. Available: <http://linkinghub. elsevier.com/retrieve/pii/S0164121213002781>. Citations on pages 55, 60, 113, 114, 136 e 137.

LOCHAU, M.; SCHAEFER, I.; KAMISCHKE, J.; LITY, S. Incremental model-based testing of delta-oriented software product lines. In: International Conference on Tests and Proofs (TAP). [S.1.]: Springer, 2012. v. 7305, p. 67-82. ISBN 9783642304729. ISSN 03029743. Citations on pages 20, 55 e 136.

LUO, G.; PETREnKO, A.; PETRENKO, R.; BOCHMANN, G. V. Selecting Test Sequences For Partially-Specified Nondeterministic Finite State Machines. In: International Federation for Information Processing (IFIP). [S.1.]: Springer, 1994. p. 91-106. Citations on pages 45, 77, 102, 116, 135 e 136 . 
MATHUR, A. P.; WONG, W. E. An empirical comparison of data flow and mutation-based test adequacy criteria. Software Testing, Verification and Reliability, v. 4, n. 1, p. 9-31, 1994. ISSN 09600833. Available: <http://doi.wiley.com/10.1002/stvr.4370040104>. Citation on page 31.

MAYRHAUSER, A. von; ZHANG, N. Automated regression testing using DBT and Sleuth. Journal of Software Maintenance: Research and Practice, v. 11, n. 2, p. 93-116, Mar. 1999. ISSN 1040550X. Available: <http://doi.wiley.com/10.1002/(SICI) 1096-908X(199903/04)11:2<93::AID-SMR188>3.3.CO;2-X>. Citation on page 30.

MCGREGOR, J. D. Testing a Software Product Line. [S.l.], 2001. 68 p. Available: $<$ http://www.sei.cmu.edu/reports/01tr022.pdf $>$. Citations on pages 11, 26, 27, 51 e 56.

MOURA, L. de; BJØRNER, N. Z3: An Efficient SMT Solver. In: 14th International Conference on Tools and Algorithms for the Construction and Analysis of Systems (TACAS). Springer, 2008. p. 337-340. Available: <http://link.springer.com/ 10.1007/978-3-540-78800-3_24>. Citations on pages 38, 106 e 108.

MUCCINI, H.; HOEK, A. V. D. Towards Testing Product Line Architectures. In: International Workshop on Testing and Analysis of Component Based Systems. [S.l.]: Elsevier, 2003. p. 111-121. Citation on page 30.

MYERS, G. J.; SANDLER, C.; BADGETT, T.; THOMAS, T. M. The Art of Software Testing. 2. ed. John Wiley \& Son, 2004. 151 p. ISSN 0960-0833. ISBN 0-471-46912-2. Available: <http://doi.wiley.com/10.1002/stvr.322>. Citations on pages 25, 29, 32, 33 e 34 .

OMG. OMG Unified Modeling Language. Version 2.5. [S.l.], 2015. 794 p. Available: <http://www.omg.org/spec/UML/>. Citations on pages 89 e 90.

OSTER, S.; MARKERT, F.; RITTER, P. Automated incremental pairwise testing of software product lines. In: International Conference on Software Product Lines (SPLC). [S.l.]: Springer, 2010. v. 6287, p. 196-210. ISBN 3642155782. ISSN 03029743. Citations on pages 20 e 54 .

OSTER, S.; WUBBEKE, A.; ENGELS, G.; SCHURR, A. A Survey of Model-Based Software Product Lines Testing. In: Model-Based Testing for Embedded Systems. [S.l.]: CRC, 2012. chap. 13, p. 338-381. Citations on pages 20, 69 e 137.

Model-Based Testing for Embedded Systems. [S.1.]: CRC, 2012. Citations on pages 20 e 39 .

PAP, Z.; SUBRAMANIAM, M.; KOVÁCS, G.; NÉMETH, G. Á. A Bounded Incremental Test Generation Algorithm for Finite State Machines. In: Testing of Software and Communicating Systems. Springer, 2007. p. 244-259. Available: < http://link.springer. com/10.1007/978-3-540-73066-8_17>. Citations on pages 38, 113, 117 e 136.

PéREZ, A. M.; KAISER, S. Bottom-up reuse for multi-level testing. Journal of Systems and Software, Elsevier, v. 83, n. 12, p. 2392-2415, 2010. ISSN 01641212. Available: $<$ http://linkinghub.elsevier.com/retrieve/pii/S0164121210001895>. Citation on page 52. 
PERROUIN, G.; SEN, S.; KLEIN, J.; BAUDRY, B.; Le Traon, Y. Automated and scalable T-wise test case generation strategies for Software Product Lines. In: 3rd International Conference on Software Testing, Verification and Validation (ICST). IEEE, 2010. p. 459-468. ISBN 9780769539904. Available: <http://ieeexplore.ieee.org/lpdocs/epic03/ wrapper.htm?arnumber $=5477055>$. Citation on page 54 .

POHL, K.; BÖCKLE, G.; LINDEN, F. van der. Software Product Line Engineering: Foundations, Principles and Techniques. [S.l.]: Springer, 2005. 1-494 p. Citations on pages 11, 19, 47, 48 e 54 .

POHL, K.; METZGER, A. Software product line testing. Communications of the ACM, ACM, v. 49, n. 12, p. 78-81, 2006. ISSN 00010782. Available: <http://www.scopus.com/inward/record.url?eid=2-s2.0-33751575661 \{\&\}partnerID=

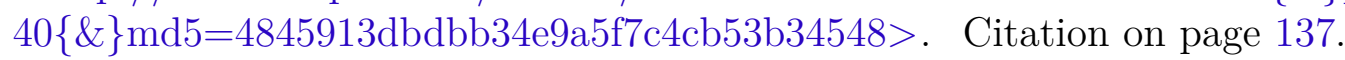

PRENNINGER, W.; PRETSCHNER, A. Abstractions for Model-Based Testing. Electronic Notes in Theoretical Computer Science, v. 116, p. 59-71, Jan. 2005. ISSN 15710661. Available: <http://linkinghub.elsevier.com/retrieve/pii/S1571066104052776>. Citation on page 39.

PRESSMAN, R. S. Software Engineering - A Practitioner's Approach. 4. ed. [S.l.: s.n.], 1997. 161 p. Citations on pages 32 e 33.

PRETSCHNER, A.; PHILIPPS, J. Methodological issues in model-based testing. In: Model-Based Testing of Reactive Systems. [S.1.]: Springer, 2004. p. 281-291. Citations on pages 11 e 36 .

RAMLER, R.; PUTSCHOGL, W. Reusing Automated Regression Tests for Multiple Variants of a Software Product Line. In: 2013 IEEE Sixth International Conference on Software Testing, Verification and Validation Workshops. IEEE, 2013. p. 122-123. ISBN 978-0-7695-4993-4. Available: <http://ieeexplore.ieee.org/lpdocs/epic03/wrapper. htm?arnumber $=6571619>$. Citation on page 53 .

RAPPS, S.; WEYUKER, E. Selecting Software Test Data Using Data Flow Information. IEEE Transactions on Software Engineering, IEEE, SE-11, n. 4, p. 367-375, 1985. ISSN 0098-5589. Available: <http://ieeexplore.ieee.org/lpdocs/epic03/wrapper.htm? arnumber $=1702019>$. Citation on page 32 .

REMMEL, H.; PAECH, B.; BASTIAN, P.; ENGWER, C. System Testing a Scientific Framework Using a Regression-Test Environment. Computing in Science \& Engineering, v. 14, n. 2, p. 38-45, mar 2012. ISSN 1521-9615. Available: <http: //ieeexplore.ieee.org/lpdocs/epic03/wrapper.htm?arnumber $=6086527>$. Citation on page 55 .

ROTHEnBERTG, J. The Nature of Modeling. Santa Monica, California, 1989. 18 p. Available: <www.rand.org/pubs/notes/2007/N3027.pdf>. Citation on page 35.

RUNESON, P.; ENGSTROM, E. Software Product Line Testing: A 3D Regression Testing Problem. In: 5th International Conference on Software Testing, Verification and Validation (ICST). IEEE, 2012. p. 742-746. ISBN 978-0-7695-4670-4. Available: $<$ http://ieeexplore.ieee.org/lpdocs/epic03/wrapper.htm?arnumber=6200179>. Citations on pages $11,20,51,53$ e 55 . 
SCHAEFER, I.; RABISER, R.; CLARKE, D.; BETTINI, L.; BENAVIDES, D.; BOTTERWECK, G.; PATHAK, A.; TRUJILLO, S.; VILLELA, K. Software diversity: state of the art and perspectives. International Journal on Software Tools for Technology Transfer, Springer, v. 14, n. 5, p. 477-495, 2012. ISSN 1433-2779. Available: <http://link.springer.com/10.1007/s10009-012-0253-y>. Citations on pages 136 e 137.

SCHIRMEIER, H.; SPINCZYK, O. Challenges in Software Product Line Composition. In: 42nd Hawaii International Conference on System Sciences. IEEE, 2009. p. 1-7. ISBN 978-0-7695-3450-3. Available: <http://ieeexplore.ieee.org/lpdocs/epic03/wrapper. htm?arnumber $=4755817>$. Citation on page 20 .

SCHLäPFER, M.; EGEA, M.; BASIN, D.; CLAVEL, M. Automatic Generation of SecurityAware GUI Models. In: European Workshop on Security in Model Driven Architecture (SECMDA). [S.l.: s.n.], 2009. p. 42-56. Citation on page 35.

SCHOBBENS, P. Y.; HEYMANS, P.; TRIGAUX, J. C. Feature Diagrams: A Survey and a Formal Semantics. In: 14th International Conference on Requirements Engineering (RE). [S.l.]: IEEE, 2006. p. 139-148. ISBN 0-7695-2555-5. Citations on pages 48 e 49 .

SEGURA, S.; GALINDO, J. A.; BENAVIDES, D.; PAREJO, J. A.; RUIZ-CORTÉS, A. BeTTy: Benchmarking and Testing on the Automated Analysis of Feature Models. In: 6th International Workshop on Variability Modelling of Software-intensive Systems (VaMoS). [S.1.]: ACM, 2012. p. 63-71. Citation on page 118.

SEI. A framework for software product line practice. 2011. Available: <http:// www.sei.cmu.edu/productlines/tools/framework/>. Citations on pages 11, 49 e 90.

Silveira Neto, P. A. M.; MACHADO, I. C.; CAVALCANTI, Y. C.; ALMEIDA, E. S. de; GARCIA, V. C.; MEIRA, S. R. d. L. A Regression Testing Approach for Software Product Lines Architectures. In: Fourth Brazilian Symposium on Software Components, Architectures and Reuse. IEEE, 2010. p. 41-50. ISBN 978-1-4244-8707-3. Available: $<$ http://ieeexplore.ieee.org/lpdocs/epic03/wrapper.htm?arnumber=5631684 $>$. Citation on page 55 .

SIMAO, A.; PETRENKO, A. Fault Coverage-Driven Incremental Test Generation. The Computer Journal, Oxford University, v. 53, n. 9, p. 1508-1522, 2010. ISSN 0010-4620. Citations on pages 21, 44, 45, 60, 61, 62, 66, 117 e 134 .

TEVANLINNA, A.; TAINA, J.; KAUPPINEN, R. Product family testing. ACM SIGSOFT Software Engineering Notes, ACM, v. 29, n. 2, p. 1-12, 2004. ISSN 01635948. Citations on pages 20 e 51.

THÜM, T.; KÄSTNER, C.; BENDUHN, F.; MEINICKE, J.; SAAKE, G.; LEICH, T. FeatureIDE: An Extensible Framework for Feature-Oriented Software Development. Science of Computer Programming, Elsevier, v. 79, p. 70-85, 2014. Citations on pages 105, 126 e 137.

UTTING, M.; LEGEARD, B. Practical Model-Based Testing: A Tools Approach. [S.1.]: Morgan Kaufmann, 2006. 456 p. Citations on pages 37, 38, 60 e 115. 
UTTING, M.; PRETSCHNER, A.; LEGEARD, B. A taxonomy of model-based testing approaches. Software Testing, Verification and Reliability, v. 22, n. 5, p. 297-312, aug 2012. ISSN 09600833. Available: <http://www.scopus.com/inward/record.url?eid= 2-s2.0-84862325128 $\{\&\}$ partnerID=tZOtx3y1>. Citations on pages 35, 36 e 39.

UZUNCAOVA, E.; GARCIA, D.; KHURSHID, S.; BATORY, D. Testing Software Product Lines Using Incremental Test Generation. In: 19th International Symposium on Software Reliability Engineering (ISSRE). IEEE, 2008. p. 249-258. Available: <http: //ieeexplore.ieee.org/lpdocs/epic03/wrapper.htm?arnumber $=4700330>$. Citation on page 20.

UZUNCAOVA, E.; KHURSHID, S.; BATORY, D. Incremental Test Generation for Software Product Lines. Software Engineering, IEEE Transactions on, IEEE, v. 36, n. 3, p. 309-322, 2010. ISSN 0098-5589. Available: <http://ieeexplore.ieee.org/lpdocs/epic03/ wrapper.htm?arnumber $=5456077>$. Citations on pages 54, 60, 113 e 136 .

VARSHOSAZ, M.; BEOHAR, H.; MOUSAVI, M. R. Delta-Oriented FSM-Based Testing. In: International Conference on Formal Engineering Methods (ICFEM). [S.l.]: Springer, 2015. v. 9407, p. 366-381. Citations on pages 136 e 137.

WEISSLEDER, S. Test Models and Coverage Criteria for Automatic ModelBased Test Generation with UML State Machines. 269 p. Phd Thesis (PhD Thesis) - Berlin, 2009. Citations on pages 11, 35 e 36.

WEISSLEDER, S.; SOKENOU, D.; SCHLINGLOFF, B.-H. Reusing state machines for automatic test generation in product lines. In: Model-Based Testing in Practice (MoTiP). Fraunhofer IRB Verlag, 2008. p. 1-10. Available: <http://citeseerx.ist.psu.edu/ viewdoc/download?doi=10.1.1.169.5699 $\{\&\} \mathrm{rep}=\operatorname{rep} 1\{\&\}$ type $=\operatorname{pdf}\{\#\}$ page $=21>$. Citations on pages 20 e 137 .

WONG, W. E.; MATHUR, A. P. Fault detection effectiveness of mutation and data flow testing. Software Quality Journal, v. 4, n. 1, p. 69-83, Mar. 1995. ISSN 0963-9314. Available: <http://link.springer.com/10.1007/BF00404650>. Citation on page 31.

YOO, S.; HARMAN, M. Regression testing minimization, selection and prioritization: a survey. Software Testing, Verification and Reliability, v. 22, n. 2, p. 67-120, Mar. 2012. ISSN 09600833. Available: <http://doi.wiley.com/10.1002/stv.430>. Citations on pages 29 e 30 . 
AGM Arcade Game Maker

BCS Body Comfort System

CFC-SM Configurable Feature-based full Coverage testing of State Machines

ConFTGen Configurable Full Test Generator

DSL Domain Specific Language

EMF Eclipse Modeling Framework

FSM Finite State Machine

FFSM Featured Finite State Machine

GEF Graphical Editing Framework

GMF Graphical Modeling Framework

IDE Integrated Development Environment

IRT-SPL Incremental Regression-based Testing for Software Product Lines

HFSM Hierarchical Featured State Machine

HSI Harmonized State Identifier

HSM Hierarchical State Machine

MBT Model-Based Testing

SAT Satisfiability

SMT Satisfiability Modulo Theory

SPL Software Product Line

SPLE Software Product Line Engineering

UI User Interface

VV\&T Verification Validation and Testing 


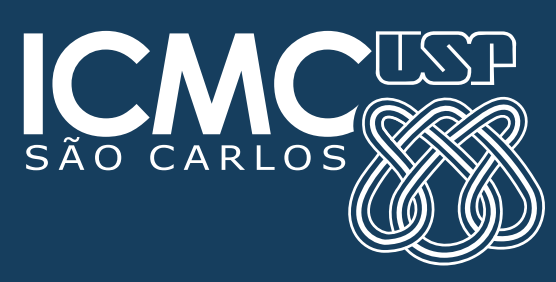

